LBL- -15902

DE83 012436

\title{
MOLECULAR PHDTOEMISSION STUDIES USING SYNCHROTRON RADIATION
}

\author{
Cariton M. Truesdale \\ Ph.D. Thesis \\ Lawrence Berkeley Laboratory \\ University of California \\ Berkeley, California 94720
}

\section{DISCLAIMER}

\begin{abstract}
This report was prepared as an account of work sponsored by an agency of the United Statex Government. Nejther the United States Government nor any agency thereof, nor any of their employees, makes any warranty, express or implied, or assumes any legal liability of responsibility for the accuracy, completeness, or useluliness of any information, apparatis, product, or process disclosed, or represents that its use would not infringe privately owned rights. Rcference herein to any specific commercial product, process, or service by trade name, trademark, manufacturer, or otherwise does not necessarily constitute or imply its endorsement, recommendation, or favoring by the United States Government or any agency thereof. The vicws and opinions of authors expressed herein do not necessarily state or rellect those of the Uniled States Government or any agency thereof.
\end{abstract}

This work was supported by the Director, Office of Energy Research, Office of Basic Energy Sciences, Materials Sciences Division of the U. S. Department of Energy under Contract No. DE-AC03-76SF00098. 
Table of Contents

Page

Abstract. .............................. vii

I. Theory

A. Intrüduction.................... 1

B. Photoelectron Angular Distributions .......... 3

C. Auger Electron Angular Distributions......... 7

References .................. 11

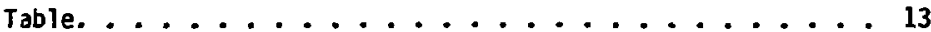

II. Experiment

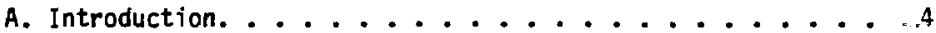

B. Properties of the Synchrotran Radiation Source. . . . . 14

C. Time-of-Flight Measurements and Analysis........ 15

D. Time-of-Flight Programing. .......... 18

References ................. 27

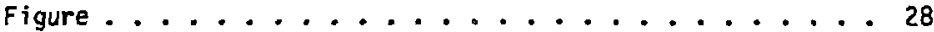

III. Angular Distributioris of $\mathrm{H}_{2} \mathrm{O}$

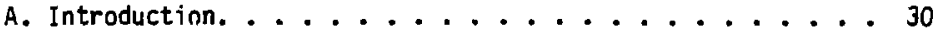

B. Results ...................... 33

C. Conclusions ................. 40

References .................. 42

Tables.......................... 44

Figures..................... 46 
Page

IV. Photoelectron Angular Distributions of the $\mathrm{N}_{2} \mathrm{O}$ Outer Valence Orbitals in the 19-31 eV Photon Energy Range
A. Introduction.
B. Results ...................... 54
C. Conclusions ...................... 63
References ................... 65
Tables.............................. 68
Figures.......................... 71

v. Vibrationally-Angle-Resolved Photoelectron Studies of $\mathrm{CO}_{2}$ and OCS

A. Introduction................. 83

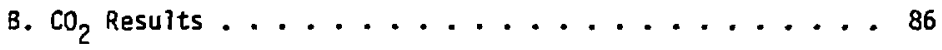

C. OCS Pesults . . . . . . . . . . . . . 94

References ................... 101

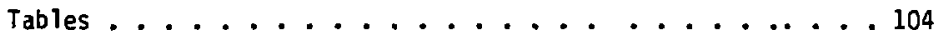

figures.......................... 107

VI. Core Level Photaelectron and Auger Shape Resonance Phenomena

In $\mathrm{CO}, \mathrm{CO}_{2}, \mathrm{CF}_{4}$, and $\mathrm{OCS}$

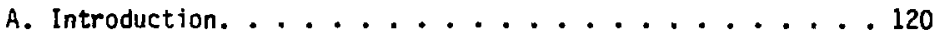

B. CO Results. . . . . . . . . . . . . 126

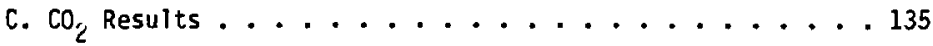

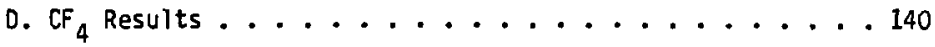

E. OCS Results . . . . . . . . . . . . 143 


$$
y / 17
$$

Page

F. Conctustions . . . . . . . . . . . 153

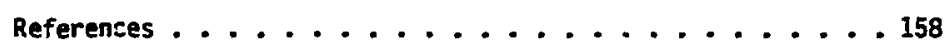

Tables . . . . . . . . . . . . . . . . 162

figures. . . . . . . . . . . . . . . 167

Acknowledgements . . . . . . . . . . . . 198 


\title{
MOLECULAR PHOTOEMISSION STUDIES
}

USING SYNCHROTRON RADIATION

\author{
Carlton Maurice Truesdale \\ Materials and Molecular Research Division \\ Lawrence Berkeley Laboratory \\ and \\ Department of Chemistry \\ University of California \\ Berkeley, California 94720
}

Abstract

The angular distributions of photoelectrons and Auger electrons were measured by electron spectroscopy using synchrotron radiation. The experimental results are compared with theoretical calculations to interpret the electronic behavior of photoionization for molecular systems.

The synchrotron radiation source provides a time structure which allows the collection of time-of-flight (TOF) spectra. The photoelectron spectroscopy using the double-angle-time-of-flight method is discussed. Two advantages of this technique are the increased electron detection efficiency and a decreased systematic error. Automated control of the experimental data collection and on-1ine data analysis provide many advantages, such as increased ease of data acquisition and manipulation.

The photoelectron partial cross sections and asymmetry parameters $B$ for the valence orbitals of $\mathrm{H}_{2} \mathrm{O}$ were measured in the energy range $h v=18-32 \mathrm{eV}$. The measurements are compared with earlier data and also multiple-scattering (MSMXa), atomic-extrapolation (AE), and Stieltjes-Tchebycheff moment-imaging (STMT) calculations. The 


\section{viij}

multiple-scattering model calculations agree well with our measurements of the photoelectron asymetries $B$ for the $1 b_{1}, 3 a_{1}$, and $1 b_{2}$ molecular orbitals of $\mathrm{H}_{2} \mathrm{O}$. The atomic character of the molecular orbitals is discussed in the context of the photoelectron angular asymmetry parameter $B$.

The vibrationally averaged photoelectron partial cross sections and asymetry parameters 8 for the four outer valence orbitals of $\mathrm{N}_{2} \mathrm{O}(X, A, B$, and $C)$ were measured over $h_{v}=19-31 \mathrm{eV}$. Vibrationally resolved data for the $A$ and $C$ ionic states were also obtained. The results are compared with previous measurements and multiple-scattering model calculations. A state-to-state comparison of the vibrationally averaged asymmetry parameters $B$ of $\mathrm{N}_{2} \mathrm{O}^{+}$and $\mathrm{CO}_{2}^{+}$was performed to show the behavior for these two isoelectronic triatomics. Similar changes in the asymmetry parameter are observed.

$V$ ibrationally resolved measurements of the $1 \pi_{u}$ molecular orbital of $\mathrm{CO}_{2}$ over $h_{v}=18-26 \mathrm{eV}$ were carried out. The vibrational states show shape resonances that are displaced in kinetic energy because of the different binding energies associated with each vibrational mode. Vibrationally resolved data for the 80 molecular orbital of ocs were also obtained. The behavior of all but the $(1,0,0)$ vibrational state of the $80^{-1}$ are similar. The iesults for $\mathrm{CO}_{2}$ are compared with other synchrotron measurements, $(e, 2 e)$ electron impact results, resonance-line measurements, and the vibrationally resolved multiple-scattering model calculations. The OCS results are compared with other 
synchrotron measurements, and vibrationally averaged measurements of oCS are compared with theoretical predictions based on the multiplescattering mode1.

Auger electron and photoelectron cross sections and asymetry parameters were measured in the studies of the inner shell ionizations for $\mathrm{CO}, \mathrm{CO}_{2}, \mathrm{CF}_{4}$, and OCS in the vicinity of the carbon (1s), oxygen (1s), and sulfur $L_{2,3}$ (2p) edges. The branching ratio for the $01 s$ shake-up states of $\mathrm{CO}$ and $\mathrm{CO}_{2}$, and the $\mathrm{S2p}$ shake-up states of OCS were also measured. Molecular shape resonances were observed for all of the carbon $\mathrm{K}$-shell studies. Comparisons are made between other measurements and multiple-scattering, Stieltjes-Tchebycheff moment imaging, Hartree-Fock static exchange, and configuration interaction calculations. There are instances where each of these theoretical models has some success in predicting many of the observed molecular effects, such as shape resonances and the intensity of satellite-line intensities. To help explain the photoionization of the S2p level of OCS, "quasi-atomic" calculations have been performed. The S2p quasi-atomic calculations may suggest that correlation effects exist between the shake-up states and the $52 p$, because of the peculiar behavior in the S2p asymmetry parameter 8 near the ionization thresholds of $S 2 p$ shake-up states.

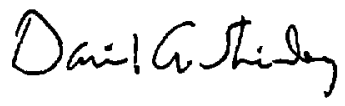




\section{Theory}

Sing softly young.

Bring bountiful gifts thee that have wealth.

Give tiny praises where praise is just,

And be what is intended you to be in this world.

Commit yourself (do not overthrow),

For the scenes and sights you focus on are totally unreal.

The world is a ripple in a pond,

A note to be sung.

From the travels of CMT

\section{A. Introduction}

The character of electronic structure and the photoionization process can be fully addressed when experimental measurements, collected over a range of photon energies, are compared with predictions based on theoretical models. Tunable synchrotron radiation makes it feasible to investigate energies, intensities, spin polarizations, and angular distributions of Auger electrons and photoelectrons. The measurements described in this dissertation in the tunable VUV and soft $x$-ray continua combine a synchrotron source and photoelectron spectroscopy in order to determine energy dependent photoelectron and Auger photoionization cross sections, angular distributions, and branching ratios.

Experimental measurements provide a test for the accuracy of theoretica? results. In providing valid models that describe the 
photoionization process, a requirement is for the mathematical solution to provide tractable information, so that experimental results can be interpreted. The marriage between experimental measurements and theoretical calculations will eventually lead to an accurate description of the photoionization process.

Experimental studies and theoretical calculations for molecular systems are at an early stage. Nonetheless, a Targe body of experimental data has been collected for a few molecular systems. Therefore a solid interpretation of molecular photoionization measurements awaits a clearer description to evolve from the comparision of theoretical models and experimental data. There are calculational difficulties in determining the continuum wavefunctions for multi-center systems; present models have schemes of approximation to circumvent these problems.

Measurements of photoelectron photoionization cross sections and angular distributions from the studies of $\mathrm{H}_{2} \mathrm{O}, \mathrm{N}_{2} \mathrm{O}, \mathrm{CO}_{2}$, OCS, $\mathrm{CO}$, and $\mathrm{CF}_{4}$ are included in this dissertation. Vibrationally resolved results for $\mathrm{N}_{2} \mathrm{O}, \mathrm{CO}_{2}$, and OCS were obtained. The Auger electron cross sections and angular distributions for $\mathrm{CO}, \mathrm{CO}_{2}, \mathrm{CF}_{4}$, and OCS were also measured. The cross sections of the $\mathrm{CO}$ and $\mathrm{CO}_{2} \mathrm{Ols}$ shake-up states and OCS S2p shake-up states were also obtained.

In the remainder of this chapter, theoretical background material is presented. The list of references that accompany this discussion is not mieant to be complete, but are examples to illustrate the topics presented. 
In Chapter II the experimentai apparatus and methods are described. The experimentat results are discussed and compared with theoretical conclusions in Chapter III-VI.

\section{Photoelectron Angular Distributions}

A goal of molecular calculations is the proper treatment for the dynamics of molecular photoionization. A molecule $(M)$ in the ground electronic state excited with monochromatic radiation hv of suitable energy emits an electron as shown by

$$
\left.\left.2 S_{0}+I_{M\left(\lambda_{0}\right.}\right)+h v+2 S_{c}+I_{M}+\lambda_{c}\right)+e^{-(s l)},
$$

where the $\lambda_{0}$ and $\lambda_{c}$ are the quantum numbers describing the rotational angular momentum of the initial state and ionic state projected on the molecular axis, respectively. The geometrical factors upon which the distribution of final ionic states depend are not affected by particular vibrational states of the neutral molecule and ionic state. ${ }^{1}$ Full consideration for vibrationally resolved ionic states demonstrates that nuclear effects are apparent. ${ }^{2}$ In the Born-Oppenheimer approximation which allows the separation of nuclear and electronic motion, the rotational quantuin numbers of the ionic core need not to be considered when rotational structure is not resolved. ${ }^{3}$ A fuli treatment of molecular rotation for diatomic molecules is given by Buck ingham. ${ }^{3}$

Tunable radiation combined with photoelectron spectroscopy allows information to be derived from electronic structure of molecules 
(atoms) by the measurement of ionization energies for the orbitals. The difference in energy between the final ionic state and initial state is called the ionization energy I. The kinetic energy a of ejected electrons and ionization energy I are restricted by the conservation of energy as shown by

$$
\text { hv }=I+c
$$

Angular momentum $(j)$ and parity $(\pi)$ conservation lead to other restrictions $\mathrm{placed}$ on the ionization process as shown by the following equations:

$$
\begin{aligned}
& h_{v}\left(j_{Y}=1, \pi_{Y}=-1\right)+M\left(j_{0}, \pi_{0}\right)+M^{+}\left(j_{C}, \pi_{C}\right)+e^{-}\left(s \ell, \pi_{e}=(-1)^{\ell}\right) \\
& \vec{j}=\vec{j}_{Y}+\vec{j}_{0}=\vec{j}_{C}+\vec{s}+\vec{l} \\
& \pi=\pi_{Y} \pi_{0}=\pi_{c} \pi_{e}
\end{aligned}
$$

The photon has values $j_{Y}=1$ and $\pi_{Y}=-1$ to specify electric dipole interaction during the absorption via collision with the target M. The parity of the photon is equal to -1 because the parity of a particle is defined as $(-1)^{2} .^{4}$ The photoelectron spin $s$ will be neglected in the remainder of the discussion because the electron spin is not observed.

Two cases need to be discussed in identifying the parity conserved values of the asymptotic photoelectron angular momentum $\mathcal{L}$. In the first case, the initial state and final state parities $\pi_{0}$ and 
$\pi_{c}$ are identical (equal symmetry), such that $\ell$ takes add values of angular momentum $(\ell=1,3,5, \ldots)$. In the second case, the initial state and final state have different parities, and $\ell$ takes even values of angular momentum $(\ell=0,2,4, \ldots)$. For a thorough treatment of angular momentum topics refer to :.ink and Satchler. 5

The photoionization cross section and photoelectron angular distribution (spin-unobserved) are additional parameters necessary to describe the photoionization process. ${ }^{6}$ In the electric-dipole approximation, the differential cross section $d \sigma / d \Omega$ of molecular (atomic) photoionization for a randomiy oriented larget (molecule or atom) which is excited by 100 percent linearly polarized radiation is given by

$$
\frac{d \sigma(c, \theta)}{J_{S}}=\frac{\sigma(\varepsilon)}{4 \pi}\left[1+B(\varepsilon) P_{2}(\cos \theta)\right] .
$$

The differential cross section $d \sigma / d \Omega$ is a function of the kinetic energy $\varepsilon$ of the photoelectron measured at an angle $\theta$ between the photoelectron emission referenced to the photon polarization axis. $7,8 p_{2}(\cos \theta)$ is the second Legendre polynomial given as $\frac{3}{2} \cos ^{2} \theta-\frac{1}{2}$. The angular distribution is completely described by the asymmetry parameter $\Delta(c)$. The is ymmetry parameter has physical solutions when the cross section has non-negative values, so that $B(\varepsilon)$ is restricted to the range $(-1 \leq 8(c) \leq 2)$. For low photon energies Eq. (6) is quite accurate. At higher photon energies, where the 
photon momentum is on the order of the photoelectron monentum or the photon wavelength is nearly equal to the atomic dinensiolis $(\sim \dot{A})$, additional multipole components are needed to describe the angular distribution. 9,10

The electronic structure of a molecule (atom) is sensitively described by measured cross sections and asymetry parameters $B(c)$. The formulation of photoionization dynamics usually necessitates a definition of the continuum wavefunction, which is expanded in terms of alternate values of orbital angular momentum $\ell$, partial-wave amplitudes, and interference terms. The cross section $\sigma(\varepsilon)$ is found to be only dependent upon the magnitude of the partial-wave amplitudes given by the squares of the dipole matrix elements. Yet, the asymmetry parameter $B(\varepsilon)$ is in addition dependent on the interference caused by non-Coulomb and Coulomb relative phase-shift differences and the signs of the dipole matrix elements.

Two energy domains characterize the angular distribution of photoelectrons. First, for non-resonant photoionization, the rapid variation in the asymmetry parameter $B(\varepsilon)$ has been shown to be caused by the Coulomb phase-shift differences of the continuum waves. 11 Secondly, in the resonant case (excluding autoionization) variation in the asyrmetry parameter $B(c)$ is attributable to variations in the dipole matrix elements. ${ }^{11}$

It has been shown that an alternative description of angular momentum dynamics can be achieved by defining the angular momentum transfer jt. Upon defining the angular momentum transfer jt, 
bookkeeping of allowed momentum states for Eq. (4) is simplified. 12,13 The angular momentum transfer $\mathrm{jt}$ is the angular momentum vector difference taken between the final ionic state and the initial state or the photoelectron $(l)$ and the photon (1), shown by the following equation.

$$
\overrightarrow{j t}=\vec{j}_{c}-\vec{j}_{0}=\vec{t}-\vec{i}
$$

Transitions which have odd values of the sum $j t+\ell+j_{Y}$ are "parity favored" transitions. Even values for the sum $j t+\ell+j_{Y}$ refer to "parity infavcred" transitions. The asymmetry parameter for parity unfavored transitions is always equal to -1.12

The molecular symmetry requirements for molecular photcionization have been used to determine the selection rules governing $j t^{14}$ based on the character of the symmetry group. In that way, the role of symetry properties in characterizing the angular distributions has been explicitly formulated for the direct ionization model. Table I. iists a few molecular point groups and the forbidden $j t$ given by Druger. 14

\section{Auger Electron Angular Distributions}

The present discussion will consider the ionization of K-shells for molecular systems. The vacancies produced in inner shells of molecules decay by an Auger process. The Auger decay accompanies the filling of the vacancy by an outer or inner valence electron. For photon energies that are insufficient to ionize core levels, excited electrons occupy discrete levels. After yalence ievels fill the 
initial vacancy, singly-charged ionic states are subsequently produced. Doubly charged ions are produced when the photoexcitation energy is sufficient to ionize core levels. Molecular valence Auger spectra have been used to identify and analyze the elemental composition of 1 arge systems. Analys is of these spectra allows the possibility for unraveling finai state assignments using atomic nomenclature, because core-level type Auger exhibit essentially atomic character. 15 Molecular Auger spectra have been studied experimentally 17-18 and theoretically. 15,19-23

The kinetic energy of the ejected Auger electron $c_{A}$ (re) laxation neglected) is given by 21

$$
\varepsilon_{A}=I_{c}-I_{j}-I_{k}-U_{e f f},
$$

where $I_{c}, I_{j}$, and $I_{k}$ are the ionization potentials of the core, $j$ th and $k$ th valence levels, respectively. $U_{\text {eff }}$ is the spindependent effective hole-hole interaction in the final state and is equivalent to the difference between the entrgy of the doubly ionized system and the ground state energy with additional energy given by $I_{j}$ and $I_{k}$.

Molecular photoabsorption is expected to be highly anisotropic for discrete transitions because the: excited states have particular symmetries and energies. 24 The interpretation of molecular Auger spectra is complicated by molecular orbital relaxation, 20 localization of the final hole states, ${ }^{22}$ and hole-hole interaction in the final state. 24 
To calculate Auger electron intensities, Auger electron transition moments are required. The Auger transition momerts can be expressed in terms of Coulomb $\left(J_{a b}\right)$ and exchange $\left(k_{a b}\right)$ integrals involving the continuum orbital $\phi(\varepsilon)$, the ionized is orbital, and the final state with holes in the outer molecular orbitals $a$ and $b .{ }^{13}$ The values of these Auger matrix s'ements are expected to be independent of the photon energy because the relative phases and Coulomb interaction should be static, ${ }^{24}$ and the Auger matrix elements have been shown to be proportional to the atomic charge. 19

The orientation of the molecular excited state affects the Auger electron angular distribution because the decay of the $\mathrm{K}$-shell is a fast process compared with molecular rotation. For that reason, photoabsorption to a $\pi$ excited state creates excited molecales that are expected preferentially to orient perpendicular to the electric vector of the light, ${ }^{24}$ as for example, in $\mathrm{CO}^{25-27}$ in contrast, the o shape resonance is expected to yield molecular ions preferentially oriented barallel. 24 Therefore the energy dependence of molecular Auger electron angular distributions should provide information about the anisotropy and identification of the symmetry of discrete excited and continuum states. 24

The angular distribution of Auger electrons has been theoretically determined by $D i l l$ et al. ${ }^{24}$ to be

$$
\frac{d \sigma\left(h_{v}, \theta\right)}{d \Omega}=\frac{\sigma\left(h_{v}\right)}{\partial \pi}\left[1+B_{m}\left(h_{v}\right) A P_{2}(\cos \theta)\right],
$$

where $B_{m}(h v)$ is the orientation parameter $\left(-1 \leq B_{m} \leq 2\right)$ and $A$ is a 
constant $(-1 / 2 \leq A \leq 1)$ that is characteristic for each Auger decay and is independent of the photon energy hu. One makes the identification that the Auger eleciron asymetry parameter, $B_{A}\left(h_{v}\right)=B_{m}\left(h_{v}\right) A$, where $A$ is expected to be nonzero.

The orientation parameter $B_{m}(h v)$ determines the alignment ${ }^{28}$ dise to unequal molecular orientations, parallel and perpendicular to the photon polarization vector, and is given by

$$
\theta_{m}(h v)=\frac{2\left[0_{\sigma}^{2}(h v)-D_{\pi}^{2}(h v)\right]}{\left[D_{\sigma}^{2}(h v)+2 D_{\pi}^{2}(h v)\right]}
$$

Unlike the photoelectron asymetry parameter $B(\varepsilon)$, which includes interference between $\sigma$ and $\pi$ channels, the orientation parameter $B_{m}\left(h_{v}\right)$ measures the photoabsorption strengths ${ }^{24} D_{\sigma}$ and $D_{\pi}$, referenced to the photon polarization axis. In Chapter VI additional theoretical discussion of Auger asymetry and experimental results is presented. 


\section{References}

1. E.S. Chang, Proceedings of the Daresbury one-day meeting Feb. 16, 1978, edited by Brian D. Buck Tey, Daresbury Laboratory.

2. U. Fano and D. Di11, Phys. Rev. A 5, 185 (1972).

3. A.D. Buck ingham, B.J. Orr, and J.M. Sickel, Phit. Trans. Roy. Soc. Lond. A 268, 147. (1970).

4. R. Eisberg, R. Resnick, Quantum Mechanics of Atorns, Molecules, Solids, Nuclei, and Particles, (New York, 1974) p. 691.

5. D.M. Brink and G.R. Satchler, Angular Momentum (Oxford, London, 1968).

6. K.N. Huang, Phys. Rev. A 22, 223 (1980).

7. C.N. Yang, Phys. Rev. 74, 764 (1948).

8. J. Cooper and R.N. Zare, J. Chem. Phys. 48, 942 (1968).

9. H.K. Tseng, R.H. Pratt, S. Yu, and A. Ron, Phys. Rev. A 17, 1061 (1978).

10. U. Fano and D. Di11, Phys. Rev. A 6, 185 (1972).

11. W. Thie1, Chem. Phys. Lett. 87, 249 (1982).

12. U. Fano, Phys. Rev. 135B, 863 (1964).

13. D. Di11, Phys. Rev. A 6, 160 (1972); D. Dill, Photoionization and other Probes of Many Electron interactions, :d. by F.J. Wuilleumier, 1976.

14. S.D. Oruger, J. Chem. Phys. 64, 987 (1976).

15. H. Agren, J. Chem. Phys. 75, 1267 (1981).

16. W.E. Moddeman, T.A. Carlson, M.O. Krause, B.P. Pullen, W.E. Bull, and G.K. Schweitzer, J. Chem. Phys. 55, 2317 (1971). 
17. C.I. Campbe11, J.W. Ragers, Jr., R.L. Hance, and J.M. White, Chen. Phys. Lett. 69, 430 (1980).

18. J.C. Fuggle, in Electron Spectroscopy; Theory, Techniques and Applications, Vol. 4, edited by A.D. Baker and C.R. Brundle (Academic, New York, 1980).

19. D.R. Jennison, Phys. Rev. Lett. 40, 807 (1978).

20. D.R. Jennison, Phys. Rev. A 23, 1215 (1981).

21. D.R. Jennison, Phys. Rev. A 69, 435 (1980).

22. T.D. Thomas and P. Weightman, Chem. ihys. Lett. 81,325 (1981).

23. T.A. Kelber, D.R. Jennison, and R.R. Rye, J. Chem. Phys. $\underline{75}, 652$ (1981).

24. D. Dill, J.R. Swanson, S. Vallace, and J.L. Dehmer, Phys. Rev. Lett. $\underline{45}, 1393$ (1980).

25. J. Stöhr, K. Baberschke, R. Jaeger, R. Treichler, and S. Brennan, Phys. Rev. Lett. 47, 381 (1981).

26. G.R. Wight, C.E. Brion, and M.J. van der Wiel, J. Electron Spectrosc. Relat. Phenom. 1, 457 (1973).

27. R.B. Kay, Ph. E. van der Leeuw, and M.J. van der Wiel, J. Phys. B 10, 2513 (1977).

28. J.L. Dehmer and D. Dill, Phys. Rev. B 17, 1692 (1978). 
Table I. Character table of forbidden values of $j t$ for representative symmetries and orbitals.

\begin{tabular}{|c|c|c|}
\hline$D_{\text {onh }}$ & $\left(\Sigma^{+}\right)$ & All ever \\
\hline $\mathrm{O}_{\text {ooh }}$ & $\left(\Sigma^{-}\right)$ & 1 \\
\hline & $(\pi)$ & 0 \\
\hline $0_{\text {chh }}$ & $(\Delta)$ & 0,1 \\
\hline$c_{2 v}$ & $\left(B_{1}, B_{2}\right)$ & 0 \\
\hline$c_{3 v}$ & (E) & 0 \\
\hline$T$ & (A) & $1,2,5$ \\
\hline$T$ & (E) & $0,1,3$ \\
\hline$T$ & (T) & 0 \\
\hline
\end{tabular}




\section{Experiment}

We embark on a self discovery, And impose the restriction that

The universe is not under the command of chaos,

But directed by natural law.

\section{A. Introduction}

The molecular photoemission measurements discussed in this dissertation were all taken at the Stanford Synchrotron Radiation Laboratory (SSRL). The following discussion will describe the doubleangle-time-of-flight instrumentation, analysis, and programing used in measuring photoelectron and Auger electron angular distributions. Many components have been discussed in detail by M.G. White, R.A. Rosenberg, S. Southworth, and P.H. Kobrin. ${ }^{1-4}$

B. Properties of the Synchrotron Radiation Source

TOF measurements were performed at SSRL because of the time structure and highly polarized character of the synchrotron radiation supplied to experimental stations (beam lines). Winick ${ }^{5}$ has given a description of the properties of the electron storage ring (SPEAR) and its associated instrumentation at the SSRL facility.

Two beam lines were used to obtain TOF photoelectron and Auger spectra. The $\left(8^{\circ}\right)$ vUV beam 1 ine ${ }^{6}$ I-3 uses a Seya-Namioka monocliromator and has an operable energy range of $h_{v}=4-36 \mathrm{eV}$. The monochromator coupled with a $1200 \mathrm{line} / \mathrm{mm}$ grating and 22.5 A bandpass 
delivers a flux of $-10^{9}-10^{10}$ photons per second $/ \mathrm{cm}^{2}$ when SPEAR operates with a $10 \mathrm{ma}$ electron current. An aluminum window with a thickness of $1500 \mathrm{~A}$ was used to separate our chamber vasuum $\left(-10^{-4}\right.$ torr) from the nonochromator ultra-high vacuum $\left(-10^{-10}\right.$ torr). Soft $X$-ray measurements can be performed at the (new $4^{\circ}$ ) beam line III-1. ${ }^{7}$ Depending upon which grating is used, a grazing incidence "grasshopper" monochromator provides photon energies from $h_{v}=20-1000 \mathrm{eV}$. A $1200 \mathrm{jine} / \mathrm{mm}$ grating was used for the measurements, so that $50 \mathrm{eV}$ was the lowest attainable photon energy. Entrance and exit slits of the monochromator were adjusted to obtain a bandpass from 0.5 and $-5.0 \mathrm{eV}$. Depending upon the electron current in SPEAR and the monochromator bandpass used, the photon flux varies between $10^{8}-10^{10}$ photons per second $/ \mathrm{cm}^{2}$. On separate occasions an aluminum (1500 A thick) and vitreous carbon (1000 \& thick) windows were used to separate the chamber vacuum from the monochromator vacuum.

C. Time-of-Flight Angular Distribution Measurements and Analysis The experimental scheme is 117 ustrated in Fig. 1 . For single electron bunch operation of SPEAR, the photon pulses, which have a period of $780 \mathrm{nsec}$ and a width of $-0.3 \mathrm{nsec}$, are monochromatized before entering our chamber. At the interaction region inside the chamber, the photon beam intersects a gas sample which has been introduced by an inlet probe positioned between two TOF detectors. The electrons enitted from the sample are detected by the TOF detectors placed at angles $\theta=0^{\circ}$ and $\theta=54.7^{\circ}$ relative to the photon polarization 
direction. The electron signals are coincidenced with the signal from the electron storage ring to define the electron time-of-flight. The signals from the detectors are directed to a time-to-energy converter, and with routing electronics are stored in two quadrants of a multichannel analyzer (MCA). Additional information about the instrument can be found in Refs. 1-4.

The TOF detectors provide major advantages for measurements of cross sections $\sigma(\varepsilon)$ and asymnetry parameters $B(\varepsilon)$ at low resolution. The entire spectrum is collected simultaneousiy at each photon energy, thereby enhancing the sensitivity, and the low duty cycle at each photoelectron and Auger electron kinetic energy enhances the signal/ noise ratio.

As stated in Chapter 1, the differential cross section for excitation by plane-polarized photons takes the form

$$
\frac{d \sigma(\varepsilon, \theta)}{d \Omega}=\frac{a(\varepsilon)}{4 \pi}\left[1+B(\varepsilon) P_{2}(\cos \theta)\right]
$$

At the "magic angle" of $54.7^{\circ}, P_{2}$ vanishes and the $d \sigma / d \Omega$ measurement yields $\sigma(\varepsilon)$. Measurement of the relative intensities of electrons collected in the $0^{\circ}$ and $54.7^{\circ}$ spectra yields $B(\varepsilon)$. We note that simultaneous data collection at two angles is intrinsically a more rellable method for obtaining $\beta(c)$ than measurements based on moving a single detector to several angles. Not only are the errors in reproducing the detector $p l a c e m e n t s$ avoided, but fluctuations in photon beam intensity and sample gas density during each counting 
period are totally compensated. The remaining principal sources of systematic error - relative detector efficiency for $B(\varepsilon)$ measurements and transmission of the $54.7^{\circ}$ detector for $o(\varepsilon)$ measurements - are dealt with in a calibration procedure.

Helium, argon, krypton, xenon, and neon are used as calibration gases. The asymmetry parameters given in the literature for these gases are compared to the ratio of the intensities measured by the $0^{\circ}$ and $54.7^{\circ}$ detectors in order to determine the relative efficiency of the two detectors as a function of the kinetic energy of electrons. Literature cross sections for these gases are compared with the relative intensity measurents taken by the $54.7^{\circ}$ detector to determine the transmission of the $54.7^{\circ}$ detector over the relevant electron kinetic energy. The calibration gases and sample gases were introduced into the sample chamber through an inlet probe described previous $7 y .9$ The sample density in the interaction region was taken as being proportional to the backing pressure (nominally 3 torr), which was monitored by a capacitance manometer. ${ }^{9}$ The photon intensity was monitored by a sodium saicylate scintillator, phototube, and picoammeter. For each counting period (typically $1000 \mathrm{~s}$ ), the relative photon flux and sample pressure were integrated and stored with electronic counters.

Data reduction is straightforward because of the calibration procedure. A more detailed description of this procedure has been given by Southworth et al. ${ }^{8}$ Briefly, $s(\varepsilon)$ values are determined from the ratio of the peak areas determined from the ratio of the peak 
area measured at $0^{\circ}\left[A\left(0^{\circ}, E\right)\right]$ and $54.7^{\circ}\left[A\left(54.7^{\circ}, E\right)\right]$ and the relative collection efficiency $\left[f_{0}(\varepsilon)\right]$ of the two detectors,

$$
B_{0}(\varepsilon)=-1+\frac{A\left(0^{*}, \varepsilon\right) / A\left(54.7^{*}, \varepsilon\right)}{{ }_{0}(\varepsilon)}
$$

The ${ }_{0}(\varepsilon)$ measurements are corrected for a small unpolarized photon-beam component, for angle averaging over the finite source region and the collection solid angles, and for the difference between the measured $B(\varepsilon)$ value and that of the calibration photoelectron line. It should be noted that hecause of the internal calibration procedure performed, the derived asymmetry parameters are on ly rather weakly dependent on the degree of polarization of the radiation, on geometrical parameters of the interaction and detection system, and on certain experimental systematic errors. 8

Peak areas measured at $54.7^{\circ}$, after normalization for sample density, photon intensity, and transmission of the $54,7^{*}$ detector, were corrected in an analogous fashion to the $B(\varepsilon)$ values to yield relative partial cross sections. Thase were then scaled to yield absolute cross sections (usualiy) with the use of previously measured total cross sections.

D. Time-of-flight Programming

The experimenter can effectively handle the stress of recording data, while being able to condense those measurements into meaningful results, with the aid of automation. On the average, it is necessary for TOF experimenters working at SSRL to be able to take data for 24 
hours per day for a period of two weeks and interpret the data simultaneously. Therefore the experimenter must be prepared to be make efficient use of beam time granted, by possessing reliable hardware and effective software to control experimental data acquisition and manipulation.

The TOF experiment has evolved from being equipped with the bare necessities to a more sophisticated method for taking mecusurements and performing data analysis. In the early stages of this project, the data were stored on magnetic tape. The on-line data analysis consisted of integrating peaks from the $0^{\circ}$ and $54.7^{\circ}$ detectors by using a pen platter that digitized the background and peak locations to calculate peak areas. Al though we could detefmine relative cross sections with the peak intensities measured at $54.7^{\circ}$, the 1 imited size of memory provided by an Motorola 8080 microprocessor (8K words) did not afford us the opportunity to program withe.t severe restrictions on the size and capabilities of the software package. Presently, our 16 bit LSI-11/23 microprocessor has 54K bytes program space and a total addressable memory of $256 \mathrm{~K}$ bytes.

In the discussion to follow, a brief description will be given of some of the hardware devices used for the TOF measurenents. The discussion will concentrate on important topics that will in general guide the experimenter to produce software with simple, standardized, and understandable routines. The focus of these topics describes the development of the program called TOFCON (time-of-flight control), its structure, the inherent problems in its specific operation, and the 
considerations needed to improve its capabilities. This progran is designed to allow the experimenter to record and anaiyze photoelectron and Auger electron spectra with relative ease without sacrificing the attribites of performing rigorous data reduction. Topics that are related to program development are ciscussed to help future workers in the TOF "group" avoid some problems and pitfalls that can easily hinder one during experimental measurements, and understand the TOFCON program structure in order to be able to effectively change it without being forced to create an entirely new program.

A short list of the hardware devices (excluding signai processing) that record, store, and help in handling the data is giveil below.

1. Canberra Series $40 \mathrm{MCA}$ (multi-channel analyser).

2. Hewlett-Packard 7220A pen plotter (microprocessor-based, RS-232C interface).

3. Ortec 778 dual counter.

4. Digital LA-120 line printer.

5. Digital VT-55 terminal.

6. Data Systems Design 8" floppy disk drive.

7. Digital LSI-11/23 microprocessor.

The Canberra Series $40 \mathrm{MCA}$ records the time-of-flight spectra in two of four quadrants. With the use of Macro-11 routines supplied by the manufacturer, the MCA can be programmed to send peak locations and data to the LSI-11/23 microprocessor. The data are finally stored on double-density floppy disks. There are many static control 
capabilities of the mas. The data can be expanded, presented on a log scale, the area can be approxinated, simultaneous live display of the $0^{\circ}$ and $54.7^{\circ}$ spectrum can be presented, and regions of the spectra can be highlighted (regions of interest) to locate peaks.

One of the indispensable hardware devices for TOF studies is a plotter. The plotter produces hard copy of spectra, graphs, and figures. An added value imparted to the Hewlett-Packard 7220A plotter is the ability to be programed with the HP-GL plotter language. The pen plotter can perform tasks like drawing curyes, digitizing points and graphs, and making special symbols (e.g., greek characters).

In order to derive partial cross sections, the intensity measured by the $54.7^{\circ}$ detector must be normalized for sample density and photon flux. The sample density is monitored with a Barytron capacitance manometer, and the photon flux is detected with a sodium salicylate scintillator, phototube and picoammeter. The signals from the manometer and picoammeter are sent to separate channels of the Ortec 778 dual counter in order to store the integrated sample density and photon flux. The computer can start and stop the counter, and read the integrated sample density and photon flux for later storage with the experimental data on rloppy disk.

The data or results can be printed with the Digital $1-A-120$ line printer. This high speed $(-120$ char/sec) printer can be used to print quickly calculations and data, 50 that large amounts of time are not wasted generating our large volume of output. The LA-120 printer can also be used as a terminal. 
The Digital VT-55 terminal is used to direct processes executed by the LSI-11/23 microprocessor or other peripheral devices. The keypad can be controlled by a text editor for easy creation/modification of text, such as progran sources. The progress of tasks can be monitored by printing information to the terminal, and the experimenter can be prompted to supply additional information to complete certain menus.

The DSD 440 floppy disk system can read, write, modify, create, and delete data in the form of files. Two 512K byte floppy disks are managed by the controller. One of the disks is a system disk having rout ines for directory maintanence, program generation, and general file utilities. The other disk usually contains data of some sort andior Fortran sources.

The Digital LSI-11/23 microprocessor performs calculations and directs tasks to be carried out by appropriate hardware devices. In addition this device allows software routines of a program to be overlaid. With this feature, many routines can be added together. These routines are grouped into certain regions and segments.

The TOFCON program is command activated. The main routine requests that the user specify a valid command. A valid command is a four letter descriptor that is defined prior to program execution. The four letter comand activates a particular task or tasks to be completed. Besides having the ability to act on elementary commands, one can generate a menu consisting of a repetition of elementary commands. 
There are two modes of operation for TOFCON. Firstly, one can work in an "echo" mode. In the echo mode there are forced instances where the terminal requires the user to input information before a task can be completed. In contrast, the "no-echo" mode allows answers to questions to be predefined and supplied internally to the program. A file contains the default answers that the TOFCON program uses to determine appropriate methods of action for certain tasks. The default values also reside in memory and can be easily changed. If the user wishes to know the individual questions that are allowed to be answered by defaults, he need only type that command and the default questions and their current values can be seen on the terminal or directed to be printed by the line printer. One power of the no-echo mode is that answers to questions can be defined, so that the user can alleviate supplying monotonous and unnecessary input. An entire process can in principle take place with the user only inputting the appropriate command.

To understand the capabilities of the TOFCON program, one needs to know the specific tasks it has been designed to perform. There are three classes of operations that TOFCON can carry out. The TOFCON program can be used in conjunction with hardware devices to retrieve data stored in the MCA, analyze data, and manufacture figures. The following discussions will only give a few of the possible uses of the TOFCON program.

The first class of tasks include the transfer of data to and from the MCA. The TOFCON program can be used to transfer data from the MCA 
to floppy disk and vice versa, to retrieve the spectral peak and background locations from the MCA, and to retrieve the output of the dual counter. While data are being collected in two quadrants of the MCA, the previous spectrum can be analyzed in the other two quadrants of tie MCA or by the pen plotter. The specification for this class of operations is that data acquisition is never restricted by data manipulations.

The second group of tasiks that TOFCON can perform is data analysis. TOFCON contains routines that can determine peak areas, backgrounds, photoionization cross sections, asymetry parameters, and branching ratios. TOFCON can also be used to do peak fitting, timeto-energy conversion of spectra, ${ }^{4}$ digitization of points or graphs, and column algebra.

The last category of uses of the TOFCON program is for making figures. The natural path from obtaining data, to deriving the important results, is to present the results in a clear illustration. The TOFCON program allows one to plot graphs with variable-automatic axes and special characters. Easily understandable routines make it possible to inake figures of journal quality without requiring a draftsman to produce them. Thus time is saved and experimenters can effectively proceed from the process of taking measurements to the completion of a paper, which presents the significant experimental results.

In some instances, high-quality graphics can be generated with programs on the VAX. The VAX is 32 bit interactive minicomputer that 
contains other routines capable of data analysis and graphics. A program called RPN was written by John Barton, Charlie Bahr, students of the same research group as myself, and Dennis Trevor (former graduate student). The RPH progran stands for Reverse Polish Notation. Hithout giving a detailed explanation of the design and operations possible using this program I quote Charlie Bahr, "the general purpose Reverse Polish Notation (RPN) program residing on the VAX computer (with a condensed version capable of being operated on a DEC LSI-11/23) has been used for graphics, curve fitting, fourier analysis, spectral manipulations (suming, subtracting, smoothing, etc.), and various $1 / 0$ specific to different types of data. The command structure is very sophisticated, allowing compound commands to be defined in terms of elementary operations. RPN can be programed to perform a series of repetitive operations. The extreme generality of RPN has given it great flexibility and consistency."

Looking to the future, the TOF method can improve its capabilities in collecting data and performing data analysis by making further improvements in the TOFCON program. A major improvement in the TOF method can be achieved by allowing the experimenter the ability to take measurements in a monorhromator scanning mode. With a few modifications and additions to existing routines, one could automatically scan the monochromator and monitor the peak areas of the $0^{\circ}$ and $54.7^{\circ}$ detector as a function of photon energy. The peak locations can be determined by modifying the time-to-energy conversion 


\section{6}

routine to provide the peak locations for peaks with binding energies $E_{B}$. By including the total experimental resolution one can describe the peak shapes.

TOFCOA can become a simpler and more general program by considering oniy a few ideas. The limitations and problems associated with the TOFCON progran must be described if any effective resolution to those difficulties is achieved, so that the TOFCON can evolve into a more sophisticated sofware package. In the future the TOFCON program should be able to accept future "undefined" data characteristics and data types (e.g., 3 angle spectra). To this end, the flexibility of the program is improved.

A modification of the command structure and menu selection can also improve the general use of the TOFCON program. If commands are created that answer specific questions, unnecessary queries by the program can be reduced. To be able to access any task from any location in the program is highly desirable. With that capability, the full power of the TOFCON program can be utilized.

In summary, the continued development of the TOFCON program is encouraged. Experimenters can effectively handle the burdens of taking measurements and simultaneously analyzing the measurements with the TOFCON program. It is wise to build upon the routines of this program to create a flexible and adaptable software system by improving the command structure, and increasing the task capabilities of the TOFCON program. 


\section{References}

1. M.G. White, Ph!O. Thesis, University of California, Berkeley (1979); Lawrence Berkeley Laboratory report No. LBL-9527.

2. R.A. Rosenberg, Ph.D. Thesis, University of California, Berkeley (1979); Lawrence Berkeley Laboratory report No. LBL-8948.

3. S. Southworth, Ph.0. Thesis, University of California, Berkeley (1982); Lawrence Berkeley Laboratory report No. LBL-13512.

4. P.H. Kobrin, Ph.D. Thesis, University of California, Berkeley (1983); Lawrence Berkeley Laboratory report No. LBL-15591.

5. H. Winick, in Synchrotron Radiation Research, H. Winick and S. Doniach, eds. (Plenum, New York, 1980).

6. V. Rehn, A.D. Baer, J.L. Stanford, 0.S. Kyser, and V.O. Jones, in VUV Radiation Physics, E. Koch, R, Haense1, and C, Kunz, eds. (Pergammon-Vieweg, London/Braunswieg, 1974), p. 780 .

7. F.C. Brown, R.Z. Bachrach, and N.L. Lien, Nucl. Instrum, Meth. $152,73(1978)$.

8. S. Southworth, C.M. Truesdale, P.H. Kobrin, D.W. Lindle, W.D. Brewer, and D.A. Shirley, J. Chem. Phys. 76, 143 (1982).

9. M.G. White, R.A. Rosenberg, G. Gabor, E.D. Poliakoff, G. Thornton, S.H. Southworth, and O.A. Shirley, Rev. Sci. Instr. 吕, 1288 (1979). 
Figure Caption

Figure 1. Schenatic diagran of the double-angie-tine-of-flight experiment for measuring photoelectron and Auger electron angular distributions. The synchrotron radiation time structure is used to record time-of-flight spectra. One detector placed at $0^{\circ}$ and another detector placed at 54.7 with respect to the photon polarization axes simultaneously measure electrons. 


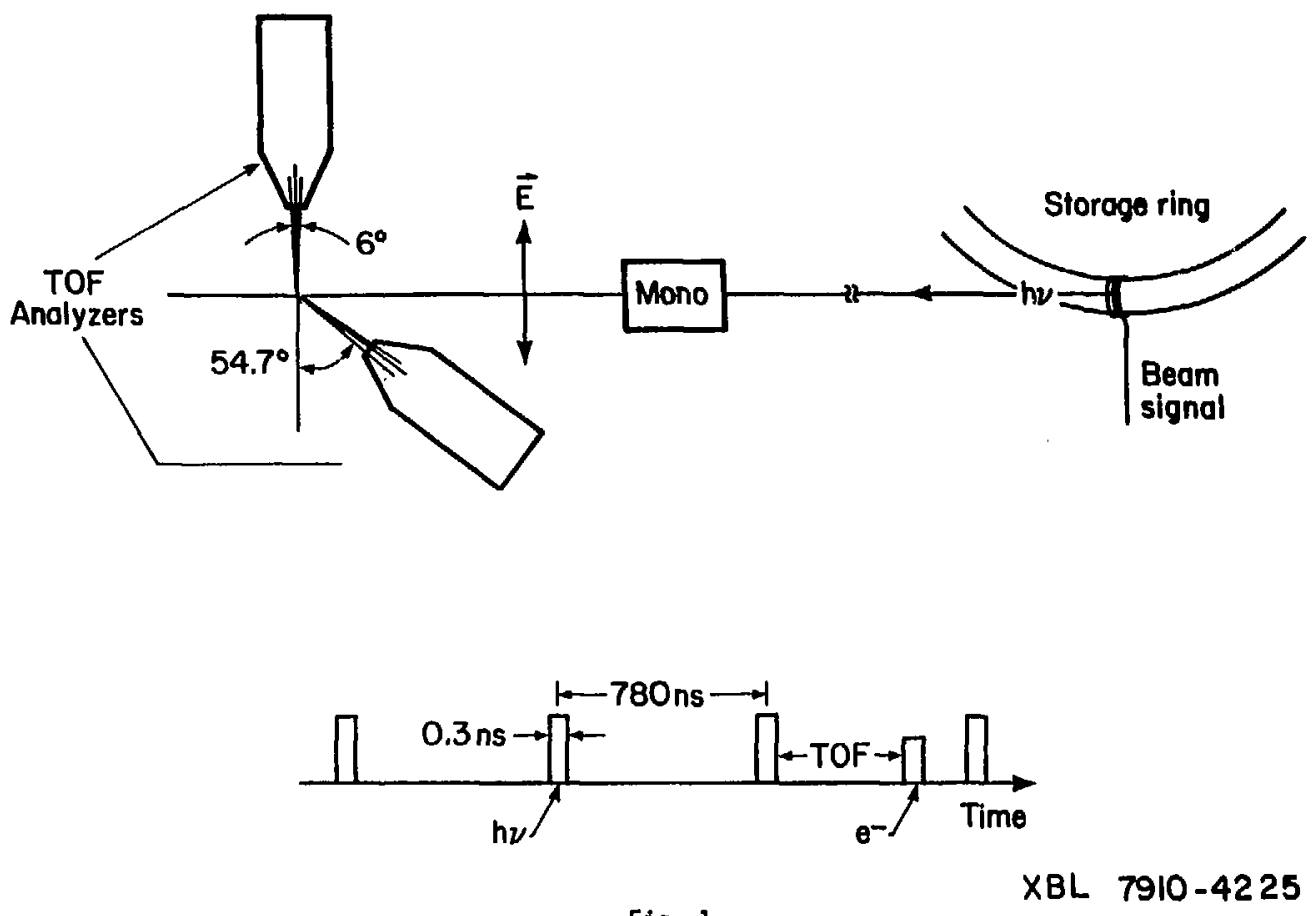

Fig. 1 
1I1. PHOTOELECTRON AMGULAR DISTRIBUTIONS OF $\mathrm{H}_{2} \mathrm{O}^{*}$

\section{A. Introduction}

Photoionization of the water molecule is a reaction of very wide interest. As in any photoionization process to which Yang's criterial of initial-state random orientation and dipole multipolarity apply, and where the spin of photoelectrons is not detected, two parameters must be measured to describe photoionization to a given ionic state through a given channel. 2 These are the angle-integrated crosssection, $O(\varepsilon)$, and the asymmetry parameter $s(\varepsilon)$, where $\varepsilon$ is the photoelectron kinetic energy. If denotes the angle between the photoelectron propagation direction and the polarization vector of the exciting radiation, the differential cross section for excitation by plane-polarized photons takes the form

$$
\frac{d o(\varepsilon)}{d \Omega}=\frac{o(\varepsilon)}{4 \pi}\left[1+B(\varepsilon) P_{2}(\cos \theta)\right] \text {, }
$$

where $P_{2}(\cos \theta)$ is the second Legendre polynomial, equal to $\frac{1}{2}\left(3 \cos ^{2} \theta-1\right)$. Thus, the photoionization of $\mathrm{H}_{2} \mathrm{O}$ can be characterized

\# C. M. Truesdale, S. Southworth, P. H. Kobrin, D. W. Lindle, G. Thornton, and D. A. Shirley, J. Chem. Phys. 76, 860 (1982). 
by measuring $[\delta \sigma(c) / \delta \Omega]$ at two or more ejection angles for the various photoionization channels, each of which corresponds to ejection of an electron from a particular molecular orbital. $B(c)$ characterizes the angular distribution of the photoelectrons. The complementary measurement of photoelectron spin polarization, in addition to $\sigma(c)$ and $B(E)$, would completely describe the photoionization process. 3

In this paper we report $\sigma(\varepsilon)$ and $\beta(\varepsilon)$ measurements for the processes

$$
\begin{aligned}
& \mathrm{H}_{2} \mathrm{O}+\mathrm{hv}_{\mathrm{v}} \rightarrow \mathrm{H}_{2} \mathrm{O}^{+}\left({ }^{2} \mathrm{~B}_{1}, 1 \mathrm{~b}_{1}^{-1}\right)+\mathrm{e}^{-} \\
& \mathrm{H}_{2} \mathrm{O}+\mathrm{hv}_{\mathrm{v}} \rightarrow \mathrm{H}_{2} \mathrm{O}^{+}\left({ }^{2} \mathrm{~A}_{1}, 3 \mathrm{a}_{1}^{-1}\right)+\mathrm{e}^{-}
\end{aligned}
$$

and

$$
\mathrm{H}_{2} \mathrm{O}+\mathrm{hv} \rightarrow \mathrm{H}_{2} \mathrm{O}^{+}\left({ }^{2} \mathrm{~B}_{2}, 1 \mathrm{~b}_{2}^{-1}\right)+\mathrm{e}^{-}
$$

for photon energies in the range $h_{v}=18-31 \mathrm{eV}$. These measurements are compared with previous experimental results and with theory.

The experiments were perforined at the Stanford Synchroton Radiation Laboratory, on the vuV beam line. 4 This source provides highly (>97 percent) ${ }^{4}$ linearly polarized radiation. The time structure of this source ( $0.3 \mathrm{nsec}$ pulse length, with a $780 \mathrm{nsec}$ period) facilitated the use of a specially designed time-of-f!igh: (TOF) detector. 5 These experiments were performed with a. $2.7 \AA$ (FWHM) mcrochromator band pass throughout the avaiiable radiation energy range of 18-31 ev. 
As calibration gases we used helium and a rare gas mixture of argon, krypton, and xenon. The asymetry parameters for these gases were taken from previous resonance-line and synchrotron-radiation measurements. ${ }^{6,7}$ Both the relative efficiencies of the two detectors and the transmission of the $54.7^{\circ}$ detactor over the ralevant electron $k$ inetic-energy range were thereby obtainea. A detailed description of the calibration procedure has been given by Southworth et al. ${ }^{8}$ The calibration gases and sample gas $\left(\mathrm{H}_{2} \mathrm{O}\right)$ were introduced into the sample chamber, through an inlet probe described previousiy. ${ }^{5}$ The photon intensity was monitored by a sodium salicylate scintillator, phototube, and picsameter. For each counting period (typically $2000 \mathrm{sec}$ ), the relative Dhoton flux and sample pressure were integrated and stored on scalers. The backing pressure for the gases was nominaliy 3 torr. A typizal TOF spectrum for the outer three molecular orbitals of $\mathrm{H}_{2} \mathrm{O}$ is shown in Fig. 1. There is good separation of the molecular orbital peaks, but vibrational structure is not resolved.

The derived relative cross sections measured by the $54.7^{\circ}$ detector, which are normalized for sample density, photon flux, and transmission of the $54.7^{\circ}$ detector, were scaled to yield absolute partial cross-sections by the use of the total photoabsorption cross- sections measured by L. de Reilhac and N. Damany ${ }^{9}$ and the ionization efficiencies reported by Katayama, et a1. 10

There are two Sections to follow. Res'jlts are presented and discussed in Section 8 and cü..clusions are given in Section $C$. 


\section{B. Results and Discussion}

At this early stage in the development of theories of nolecular photoionization, any discussion of molecular $\sigma(c)$ and $B(c)$ parameters must be somewhat tentative. Therefore, comparisions of results from different experimental and theoretical sources must perforce be an implicit evaluation of the methods employed by those sources. Thus, in the context of discussing our results we are addressing methodologies as well as the physical properties of the water molecule.

The ground-state electronic configuration of water is $\left(1 a_{1}\right)^{2}\left(2 a_{1}\right)^{2}\left(1 b_{2}\right)^{2}\left(3 a_{1}\right)^{2}\left(1 b_{1}\right)^{2}$. In the UV range available, the $1 a_{1}$ and $2 a_{1}$ electrons are too strongly bound to be ejected. The other three orbitals can be ionized according to the channels in Eq. 2. In Table I, we present the Dartial cross-sections and asymmetry parameters for tinese three channels in the photon energy range 18-31 eV. Also shown for comparison are $s(\varepsilon)$ values at hu $=21.2 \mathrm{eV}$, reported by Carison and McGuire ${ }^{11}$ and by Katsumata, Achiba, and Kimura, ${ }^{12}$ and the $\sigma(c)$ measurements at $21.2,23.5$, and $24.5 \mathrm{eV}$ reported by Katsumata, et al. and dipole $(e, 2 e)$ results of Tan, Brion, van der Leeuw, and van der Wiel. 13 The agreement is good.

Before separately discussing our results for the three channeis, we note a strong similarity among them. This arises, as Roche, Salahub, and Messmer ${ }^{14}$ have pointed out, because the $1 b_{1}, 3 a_{1}$, and $1 b_{2}$ molecular orbitals are all derived from the $02 p$ shell. Banna and Shirle; carried this analogy further in discussing photoemission from the second-row hydrides, ${ }^{15}$ noting both their conceptual 
derivation from neon and the dominant contribution of the second-row atom's valence orbitals (rather than a hydrogen "15" orbital) to the photoemission cross-sections. Roche, et al. ${ }^{14}$ computed $o(c)$ and $s(c)$ values for $\mathrm{O} 2 \mathrm{p}$ to compare with their $\mathrm{H}_{2} \mathrm{O}$ molecular-orbital $\sigma(c)$ and $B(c)$ results, which are based on the multiple-scattering $x_{\alpha}$ method. 16,17 Thus, the strong similarity among the $o(\varepsilon)$ and $\Delta(\varepsilon)$ curves (Figs. 2, 3, and 4) for the three channels is the first qualitative success of this theoretical picture. A more quantitative measure of the success of $X_{a}$ computations is obtained by comparison with other theoretical curves and with experiment, as discussed below.

The lone-pair $1 b_{1}$ orbital is least distorted from an atomic $02 p$ orbital by the presence of the atomic hydrogens because this orbital is orthogonal to the plane of the molecule and is nonbonding. As predicted, ${ }^{14}$ both $\sigma(c)$ and $s(\varepsilon)$ follow the atomic orbital curves quite closely as shown in Fig. 2. The observed -60 percent increase of $\theta(\varepsilon)$ with increasing energy agrees well with the $x_{\alpha}$ calculation, and the agreement of the $a(\varepsilon)$ behavior near the end points leaves little to be desired. The MSXa calculation predicts a decrease of $\sigma(\varepsilon)$ by about 20 percent with increasing energy, al though the absolute value of $o(\varepsilon)$ is typically 20 percent higher than predicted. There is better agreement between our $\sigma(\varepsilon)$ results and $X_{\alpha}$ calculation below $20 \mathrm{el}$ photon energy.

The magnitude of $\sigma(\varepsilon)$ was predicted more accurately by Williams and Langhoff, ${ }^{18}$ and by Hilton, Nordholm, and Hush. ${ }^{19}$ The former calculation was based on the Stieltjes-Tchebycheff (ST) moment imaging 
theory, 20,21 and the latter is developed by use of the ground state inverston potential method (GIPM). The ST approach uses variational calculations of square-integrable wavefunctions of the photoelectron interacting with a non-local and noncentral molecular-ionic potential. It yields pseudospectra and oscillator strengths from which the crosssections are derived by moment-imaging methods, but $B(\varepsilon)$ is not calculated because the continuum wavefunctions are not determined. It should, however, be capable of predicting structure in $\sigma(\varepsilon)$, a subject to be discussed latter. The ST calculation of Delaney, Saunders, and Hillier 22 is in onty qualitative agreement with our results for predicting the decrease in $\sigma(\varepsilon)$ as a function of photon energy.

The GIPM calculation determines the molecular orbital cross-sections from those of the atomic subshell components. It can include the interference effects between the atomic components of the molecular orbital. ${ }^{21}$ As stated, the calculations of Hilton, et al. predict o(c) with rather good accuracy. Their model determined that interference effects between the atomic components of the $1 b_{1}$ molecular orbital are negligible.

We conclude our comments on the gross form and magnitude of $\sigma(\varepsilon)$ for the ${ }^{2} \mathrm{~B}_{1}\left(1 b_{1}^{-1}\right)$ transition by noting that, although the $(e, 2 e)$ data do not show any resonance structure, there is otherwise good qualitative agreement between our results and the $(e, 2 e)$ data of Tan, et al. ${ }^{13}$ throughout the range of our measurements. Maxima in our $\sigma(c)$ data appear near $22 \mathrm{eV}$ and $25 \mathrm{eV}$, and some structure is present below $20 \mathrm{eV}$. Outuit, et al. reported photoelectron intensities for 
this state up to $20 \mathrm{eV}$, with structure at $19 \mathrm{eV}$, but the ir reported cross-sections are higher than ours in the small region of overlap. 23 Those authors suggest that the broad maximum at $19 \mathrm{eV}$ may be due to a competition between neutral dissociation and autoionization to jonic states of $\mathrm{H}_{2} \mathrm{O}$. Wu and Judge, who reported recent measurements of Lyman a fluorescence of neutral dissociation products of $\mathrm{H}_{2} \mathrm{O}$, have suggested that a sharp structure present in absorption spectra at $-18.5 \mathrm{eV}$ corresponds to an s-1ike Rydberg state, converging to the $2_{B_{2}}$ ionic channel, from which predissociation occurs. ${ }^{24}$ This explanation accounts for resonance structure in, and competition between, the photoionization and dissociation channels.

The two ST calculations are somewhat different in detail, one being based on the time-dependent Hartree-Fock (TDHF) approximation ${ }^{18}$ and the other on a static-exchange approximation, 22 and their resulting $\sigma(x)$ urves differ as well. In particular, excitations of virtual discrete states and states in the ionization continuum (e.g., $\left.1 b_{1} \rightarrow 4 a_{1}\right)$ in the TOHF ${ }^{18}$ calculation were found to yield maxima in the sub-channel cross sections for the ${ }^{2} B_{1}\left(1 b_{1}^{-1}\right)$ channel. These maxima were smaller or absent in their final curyes. A broad shape resonance centered around $22 \mathrm{el}$ photon energy due to excitation of a $\sigma^{*} a_{1}$ valence-like orbital predicted by Williams and Langhoff may identify the resonance structure located at $-22 \mathrm{eV}$ in the present work. Figure 2 shows that Delaney et al. 22 also find structure in the o(c) curve at -18 eV for the ${ }^{2} B_{1}$ state, but with small amplitude. The experimental response feature in $\sigma(c)$ for the ${ }^{2} B_{1}$ state below $20 \mathrm{eV}$ 
may also result from the shape resonance predicted by Delaney, et al. Until confirmatory measurements are made it seems prudent to defer further interpretation.

The $B(c)$ parameter for the ${ }^{2} \mathrm{~B}_{1}\left(1 \mathrm{~b}_{1}^{-1}\right)$ channel has been measured previously only at fixed energies (see Table I), and has been calculated only by Roche, et al. As noted above, agreement between experiment and theory is excellent, except for the structure near $h v=22 \mathrm{eV}$ and $25 \mathrm{eV}$, as in $\sigma(\varepsilon)$. In particular, $B(\varepsilon)$ starts relatively high (0.76) at $h_{v}=18 \mathrm{eV}$, rises quickly to 1.0 by $h_{v}=20 \mathrm{ev}$, and (except for the structure near $22 \mathrm{eV}$ and $25 \mathrm{eV}$ ) remains large and increases to -1.4 near $30 \mathrm{eV}$, in good accord with the $x_{a}$ results.

Results for the ${ }^{2} \mathrm{~A}_{1}\left(3 \mathrm{a}_{1}^{-1}\right)$ channel are shown in Fig. 3. There is good agreement among our measurements, the results of Dutuit, et al. ${ }^{23}$ above $22 \mathrm{eV}$, and the $(e, 2 \mathrm{e})$ results $^{13}$ fo. the entire photon energy range. This channel involves the " $02 p-1$ ike" orbital, which is inplane and directed along the $C_{2 v}$ axis. It has significant involvement with the hydrogen atoms. This fact is evident in photoemission. For example, in earlier work ${ }^{15}$ the ${ }^{2} A_{1}$ intensity was shown to increase much more between spectra excited by soft-x-ray radiation (132.3 eV) and those excited by higher energies (1487 eV) than did those of the ${ }^{2} B_{1}$ or ${ }^{2} B_{2}$ states. This suggests that $02 \mathrm{~s}$ admixture is oresent in the $3 a_{1}$ orbital. We interpret this as being due to sp hybridization, which is allowed by symmetry. Thus, unlike the $1 b_{1}$ and $2 b_{2}$ orbitals, the $3 a_{1}$ orbital does not have a nodal plane through the oxygen nucleus. 
There is little difference between the present experimental o(c) values for the ${ }^{2} B_{1}\left(1 b_{1}^{-1}\right)$ and ${ }^{2} A_{1}\left(3 a_{1}^{-1}\right)$ states, al though the calculated $\sigma(c)$ values of Roche, et al. are somewhat higher for the ${ }^{2} A_{1}$. On the average, the $X_{a}$ calculation is somewhat closer to experiment than the $5 T^{18,22}$ or $6 \mathrm{SPM}^{19}$ results. The GIPM calculation that includes interference effects for the ${ }^{2} A_{1}$ channel is presented in Fig. 3: the GIPM calculation without interference effects would be in better agreement with the present results, judging from the four points given by Hilton, et al. 19 The GiPM oicj curve shown in Fig. 3 predicts a broad maximum near $20 \mathrm{eV} .^{19}$ Delaney, et al. ${ }^{22}$ predict a resonance at $-19 \mathrm{eV}$. Williams and Langhoff ${ }^{18}$ predicted that a continuum subchannel resonance $-25 \mathrm{eV}$ is present for the excitation into the continuum o*a ${ }_{1}$ orbital. The experimental structure falls at $-18 \mathrm{eV}, \sim 22 \mathrm{eV}$, and $\sim 25 \mathrm{eV}$.

The prediction of $\mathrm{B}(\mathrm{c})$ for the ${ }^{2} \mathrm{~A}_{1}\left(3 \mathrm{a}_{1}^{-1}\right)$ ionic state is a success for the $X_{a}$ model. The theoretical curve starts at a low value of 0.6 at $h_{v}=18 \mathrm{eV}$ and rises to a value of 1.2 near $h_{v}=31 \mathrm{eV}$, in good overall agreement with the data. This behavior is to be contrasted to the $B(c)$ curve for the ${ }^{2} B_{1}\left(\mathrm{bb}_{1}^{-1}\right)$ channel discussed above. The two $B(\varepsilon)$ curves are quite different in detail, as a careful comparison will show, and on a coarse scale the predicted differences are closely followed experimentally. The difference of the ${ }^{2} A_{1}\left(3 a_{1}^{-1}\right)$ $B(\varepsilon)$ curve from that for the ${ }^{2} B_{1}\left(1 b_{1}^{-1}\right)$ state, which closely follows the differences from the $B(\varepsilon)$ curve of an $02 p$ orbital, can be attributed to perturbations of the $02 p$ orbital by the hydrogens, 
leading to hybridization. Table II presents the theoretical $B(c)$ and experimental difference between ${ }^{2} B_{1}$ and ${ }^{2} A_{1}$ ionic states of $\mathrm{H}_{2} \mathrm{O}$. Both the experimental and the theoretical average differences $\Delta B$ for these two states are $-0.3 \mathrm{~B}$ unit. The $x_{a}$ calculation is in excellent agreement with experiment in predicting this molecular bonding effect. Again weak resonance-like structure is found in $B(c)$ at $h v=20 \mathrm{eV}$ and $24 \mathrm{ev}$.

The ${ }^{2} \mathrm{~B}_{2}\left(1 \mathrm{~b}_{2}^{-1}\right)$ cross section is larger than those of the other two states as shown in Fig. 4 , wrich is cunisistent with a larger difference of this $\mathrm{O}-\mathrm{H}$ bonding orbital from an $02 \mathrm{p}$ orbital. The $\mathrm{Xa}_{a}$ calculation predicts this result well, 14 while the GIPM curve is low and the ST calculations 18,22 are typically 30-50 percent higher than experiment. Our data agree well with the $(e, 2 e)$ result:s ${ }^{13}$ and with Dituit, et al. ${ }^{23}$ There appears to be two small resonances in our data at $\sim 22 \mathrm{eV}$ and $-25 \mathrm{eV}$. Williams and Langhoff ${ }^{18}$ predict a broad subchannel resonance centered around $25 \mathrm{eV}$ due to transitions into a $\sigma^{*} a_{1}$ orbital in the continuum, but no maximum ajpears in the final $\sigma(\varepsilon)$ curve. Cross section measurements of Dituit, et al. seem to confirm that there is structure near $22 \mathrm{ev} .^{23}$ The ST calculations of Delaney, et al. ${ }^{19}$ also predict a resonance feature near $-23 \mathrm{eV}$.

The $B(c)$ parameter for the ${ }^{2} \mathrm{~B}_{2}\left(1 b_{2}^{-1}\right)$ channel is much lower than for the other two channels and for the $02 p$ orbital, owing to its strong molecular $\mathrm{O}-\mathrm{H}$ bonding character. The $\mathrm{B}(\boldsymbol{c})$ value increases smoothly from -0.1 to 0.6 as $h_{v}$ increases from $21 \mathrm{eV}$ to $30 \mathrm{eV}$. This behavior is well predicted by the $X_{a}$ model, which is in very good agreement at the high-energy end. 
C. Conclusions

Several conclusions can be drawn from this work:

1. The $\sigma(c)$ and $B(c)$ parameters for the ${ }^{2} B_{1}\left(1 b_{1}^{-1}\right),{ }^{2} A_{1}\left(3 a_{1}^{-1}\right)$, and ${ }^{2} \mathrm{~B}_{2}\left(1 \mathrm{~b}_{2}^{-1}\right)$ channels in the photoionization of $\mathrm{H}_{2} \mathrm{O}$ correspond with the parameters for $02 p$ orbitals from which these molecular orbital are derived. The $\sigma(\varepsilon)$ and $\beta(\varepsilon)$ parameters are sensitive to chemical bonding.

2. The $X_{a}$ calculation of Roche, et al. predicts the overall form of $\sigma(\varepsilon)$ and $s(\varepsilon)$ for these ortuttals very well.

3. The GIPM calculation of Hilton, et al. predicts $\sigma(c)$ equally well, on the average.

4. The two available ST calculations predict $\sigma(\varepsilon)$ values which tend to be somewhat higher than experiment.

5. Our work agrees well overall with the $(e, 2 e)$-derived o( $\varepsilon)$ results of Tan, et al.; however, their results do not show any structure.

6. Our measurements show weak resonance-like structure in both $\sigma(\varepsilon)$ and $B(\varepsilon)$, as do the results of Dutuit, et al. There is good qualitative agreement with Dutuit, et al. at photon energies higher than $21 \mathrm{eV}$.

7. The ST models show resonances in the ionization sub-channels, corresponding to excitation of virtual discrete orbitals imbedded in the continuum. The location of broad continuum resonances predicted by Willians and Langhoff may identify the underlying resonance structure present in our data at $22 \mathrm{eV}$ 
for the ${ }^{2} B_{1}$, and 25 eV for the ${ }^{2} A_{1}$ and ${ }^{2} B_{2}$ ionic states calculations of Delaney, et al. give further indication that the ST method may possibly determine resonance structure in the cross sections of the ionic states of $\mathrm{H}_{2} \mathrm{O}$. More experimental anf theoretical study is needed to provide a definitive understanding of the usefulness of the ST model in the prediction and identification of resonances in $\mathrm{H}_{2} \mathrm{O}$. 8. Vibrationally resolved measurements of the ${ }^{2} B_{1},{ }^{2} A_{1}$, and $2_{B_{2}}$ ionic states would heip in the identification of weak features in the data that are possibly due to autoionization, predissociation, and shape resonances. 


\section{References}

1. C. N. Yang, Phys Rev. 74, 764 (1948).

2. If direct photodissociation accompanies photoionization, there is more information in the correlation of product properties.

3. K. N. Huang, Phys. Rev. A 22, 223 (1980).

4. V. Rehn, A. D. Baer, J. L. Stanford, D. S. Kyser, and V. 0. Jones, in "Vacuum Ultraviolet Physics," edited by $E$. Koch, R. Haensel, and C. Kunz (Braunschweig, Pergammon-Vieweg, 1974), pp. 780-2.

5. M. G. White, R. A. Rosenberg, G. Gabor, E. D. Poliakoff, G. Thornton, S. H. Southworth, and D. A. Shirley, Rev. Sci. Instrum. 50, 1288 (1979).

6. W. H. Hancock and J. A. R. Samson, J. Electron Spectrosc. Relat. Phenom. 9, 211 (1976); J. Kriele and A. Schweig, J. Electron Spectrosc. Re1at. Phenom. 20, 191 (1980).

7. F. Wuilleumier and M. O. Krause, J. Electron Spectrasc. Relat. Phenom. 15, 73 (1978). J. L. Dehmer, W. A. Chupka, J. Berkowitz, and W. T. Jivery, Phys. Rev. A. 12, 1966 (1973).

8. S. Southworth, C. M. Truesdale, P. H. Kobrin, O. W. Lindle, W. D. Brewer, and D. A. Shirley, submitted to J. Chem. Phys. (1981).

9. L. de Reilhac and N. Damany, J. Quant. Spectry. Radiative Transfer 18, 121 (1977).

10. D. H. Katayama, R. E. Huffman, and L. L. O'Bryan, J. Chem. Phys. 59, 4309 (1973).

11. T. A. Carlson and G. E. MCGuire, J. Electr. Spectr. Relat. Phonom. 1. $209(1972-73)$. 
12. S. Katsumata, Y. Achiba, and K. Kimura, J. Electron. Spectrose. Relat. Phenom. 17, 229 (1979).

13. K. H. Tan, C. E. Brion, Ph. E. van der Leeuw, and M. J. van der Wie1, Chem. Phys. 29, 299 (1973).

14. M. Roche, O. R. Salahub, and P. R. Messmer, J. Elect. Spectr. and Relat. Phenom. 19, 273 (1980).

15. M. S. Banna and D. A. Shirley, J. Chem. Phys. 63, 4759 (1975).

16. J. C. Slater, Adv. Quantum Chem. 6,1 (1972).

17. D. Dill and J. Dehmer, J. Chem. Phys. 61, 692 (1974).

18. G. R. J. Williams and P. W. Langhoff, Chem. Phys. Letters 60, 201 (1979).

19. P. R. Hilton, S. Nordholm, and N. S. Hush, Chem. Phys. Letters 64, 595 (1979).

20. P. W. Langhoff, Chem. Phys. Letters 22, 60 (1973).

21. T. N. Rescigno, C. F. Bender, B. V. Mckoy, and P. W. Langhoff, J. Chen. Phys. 63, 970 (1978).

22. J. J. Delaney, Y. R. Saunders, and I. H. Hillier, J. Phys. B. Atom. Molec. Phys. 14, 819 (1981).

23. 0. Dutuit, K. Ito, A. Tabche-Foutiale, P. Morinm T. Baer, P. M. Guyon, and I. Nenner, Proc. 6th Int. Conf. of Vacuum UTtraviolet radiation Physics, Charottesville, USA Abstracts 12, 17 (1980).

24. C. Y. R. Wu and D. L. Judge, J. Chem. Phys. 75, 172 (1981). 
Table I. Cross sections and asymetry parameters of the ${ }_{B_{1}}, 2_{A_{1}}$ and ${ }_{B_{2}}$ ionfic states of $\mathrm{H}_{2} \mathrm{O}$.

\begin{tabular}{|c|c|c|c|c|c|c|}
\hline \multirow[b]{2}{*}{$h v(e V)$} & \multicolumn{2}{|r|}{${ }^{2} B_{1}$} & \multicolumn{2}{|r|}{$2_{A_{1}}$} & \multicolumn{2}{|r|}{$\mathrm{Z}_{\mathrm{B}_{2}}$} \\
\hline & $O(M b)$ & - & $O(M b)$ & $B$ & $\sigma(M b)$ & $\bullet$ \\
\hline 18. & $5.60(14)$ & $.76(5)$ & $6.51(16)$ & $.35(4)$ & $\cdots$ & $\cdots$ \\
\hline 19. & $5.91(15)$ & $.88(5)$ & $5.83(14)$ & $.45(4)$ & $\cdots$ & $\cdots$ \\
\hline 19.5 & $5.01(15)$ & $1.18(8)$ & $4.83(14)$ & $.63(6)$ & & \\
\hline 20. & $5.24(15)$ & $1.09(6)$ & $4.71(14)$ & $.85(6)$ & $\cdots$ & $\cdots$ \\
\hline 20.5 & $5.23(13)$ & $1.08(7)$ & $5.23(13)$ & $.75(5)$ & & \\
\hline 21.2 & $\begin{array}{l}5.77(14) \\
5.9(4)^{a}\end{array}$ & $\stackrel{.99(8)}{1.09(4)^{\mathrm{a}}, 1.0(1)^{\mathrm{b}}}$ & $\begin{array}{l}5.87(16) \\
6.0(4)^{d}\end{array}$ & $\begin{array}{l}.68(5) \\
.45(5)^{a} . .3(1)^{b}\end{array}$ & $\begin{array}{l}7.03(16) \\
8.4(8)^{2}\end{array}$ & $\begin{array}{l}-.11(3) \\
-.09(10)^{a},-.1(2)\end{array}$ \\
\hline 22 & $6.06(15)$ & $.96(6)$ & $5.70(15)$ & $.72(5)$ & $8.05(18)$ & $-.10(3)$ \\
\hline 23 & $5.92(15)$ & $.96(6)$ & $5.65(14)$ & $.64(5)$ & $7.32(17)$ & $-.06(3)$ \\
\hline 23.3 & $5.46(14)$ & $1.06(7)$ & $5.41(14)$ & $.76(6)$ & $7.88(18)$ & $.02(3)$ \\
\hline 23.5 & $\begin{array}{l}5.23(13) \\
6.036^{6}\end{array}$ & $1.24(7)$ & $\begin{array}{l}5.00(13) \\
5.44 C^{2}\end{array}$ & $1.00(5)$ & $\begin{array}{l}6.91(16) \\
6.63 C^{6}\end{array}$ & $.02(3)$ \\
\hline 24 & $5.11(14)$ & $1.19(7)$ & $4.77(14)$ & $.85(6)$ & $6.81(17)$ & $.06(3)$ \\
\hline 24.5 & $\begin{array}{l}5.23(13) \\
5.655^{5}\end{array}$ & $1.20(7)$ & $\begin{array}{l}4.88(13) \\
5.30^{\mathrm{C}}\end{array}$ & $.94(6)$ & $\begin{array}{l}6.69(16) \\
6.59 \mathrm{C}\end{array}$ & $.20(3)$ \\
\hline 25 & $6.16(14)$ & $.88\langle 5\rangle$ & $6.00(14)$ & $.75(5)$ & $7.83(27)$ & $.22(3)$ \\
\hline 26 & $5.55(15)$ & $1.05(5)$ & $5.03(13)$ & $.90(5)$ & $6.94(17)$ & $.23(4)$ \\
\hline 27 & $5.26(13)$ & $1.21(6)$ & $5.45(13)$ & $.80(3)$ & $6.70(15)$ & $.37(4)$ \\
\hline 28 & $4.92(14)$ & $1.31(8)$ & $5.14(14)$ & $.85(6)$ & $6.15(15)$ & $.48(5)$ \\
\hline 29 & $4.63(13)$ & $1.12(8)$ & $4.66(13)$ & $1.01(7)$ & $5.77(15)$ & $.54(5)$ \\
\hline 30 & $4.40\langle 12\rangle$ & $1.38(8)$ & $3.11(13)$ & $1.20(9)$ & $5.43(15)$ & $.55(6)$ \\
\hline 31 & $4.38(14)$ & $1.36(9)$ & $3.72(12)$ & $1.21(9)$ & $5.54(25)$ & $.56(5)$ \\
\hline
\end{tabular}

-Errors in the last place are given parenthetically.

AReference 12 .

DReference 11 .

CReference 13 . 
Table II. Asymmetry parameter differences between the ${ }^{2} \mathrm{~B}_{1}$ and $2 \mathrm{~A}_{2}$ ionic states of $\mathrm{H}_{2} \mathrm{O}$.

\begin{tabular}{|c|c|c|}
\hline hv & $\Delta B$ (experiment) ${ }^{\star}$ & $\Delta B$ (theory) ${ }^{\star \star}$ \\
\hline 18 & $.41(4)$ & .30 \\
\hline 20 & $.24(6)$ & .30 \\
\hline 22 & $.24(5)$ & .29 \\
\hline 24 & $.34(6)$ & .29 \\
\hline 26 & $.15(5)$ & .25 \\
\hline 28 & $.46(7)$ & .28 \\
\hline 30 & $.18(8)$ & .26 \\
\hline
\end{tabular}


Figure Captions

Fig. 1. TOF photoelectron spectrum showing the ${ }^{2} B_{1},{ }^{2} A_{1}$, and ${ }^{2} \mathrm{~B}_{2}$ ionic states of $\mathrm{H}_{2} \mathrm{O}$.

Fia. 2. $\sigma(\varepsilon)$ and $B(\varepsilon)$ for the ${ }^{2} B_{1}\left(1 b_{1}^{-1}\right)$. For the $\sigma(\varepsilon)$ curves, $A$ is the $X_{a}$ calculation of Roche, et al., B is the GIPM Ci culation of Hilton, et al., $C$ is the ST calculation of Williams and Langhofi, $D$ is the ST calculation of Delaney, et al., $\square$ - are the $(e, 2 e)$ data of Tan, et al., $\mathbf{D}$ - are the measurements of Dituit, et al., and are the present me ssurements. For the $B(\varepsilon)$ curves, the solid curve is the $x_{\alpha}$ calculation of Roche, et al. and are the present me :surements.

Fig. 3. Photoionization cross section $\sigma(\varepsilon)$ and photoelectron as ynmetry for $\mathrm{H}_{2} \mathrm{O}^{+} \mathrm{A}_{1}\left(\mathrm{a}_{1}^{-1}\right)$. The experimental and treoretical results are denoted as in Fig. 2.

Fig. 4. Photoionization cross section $\sigma(\varepsilon)$ and photoelectron $B(\varepsilon)$ for $\mathrm{H}_{2} \mathrm{O}^{+}{ }^{2} \mathrm{~B}_{2}\left(1 \mathrm{~b}_{2}^{-1}\right)$. The experimental and theoretical results are denoted as in Fig. 2 . 


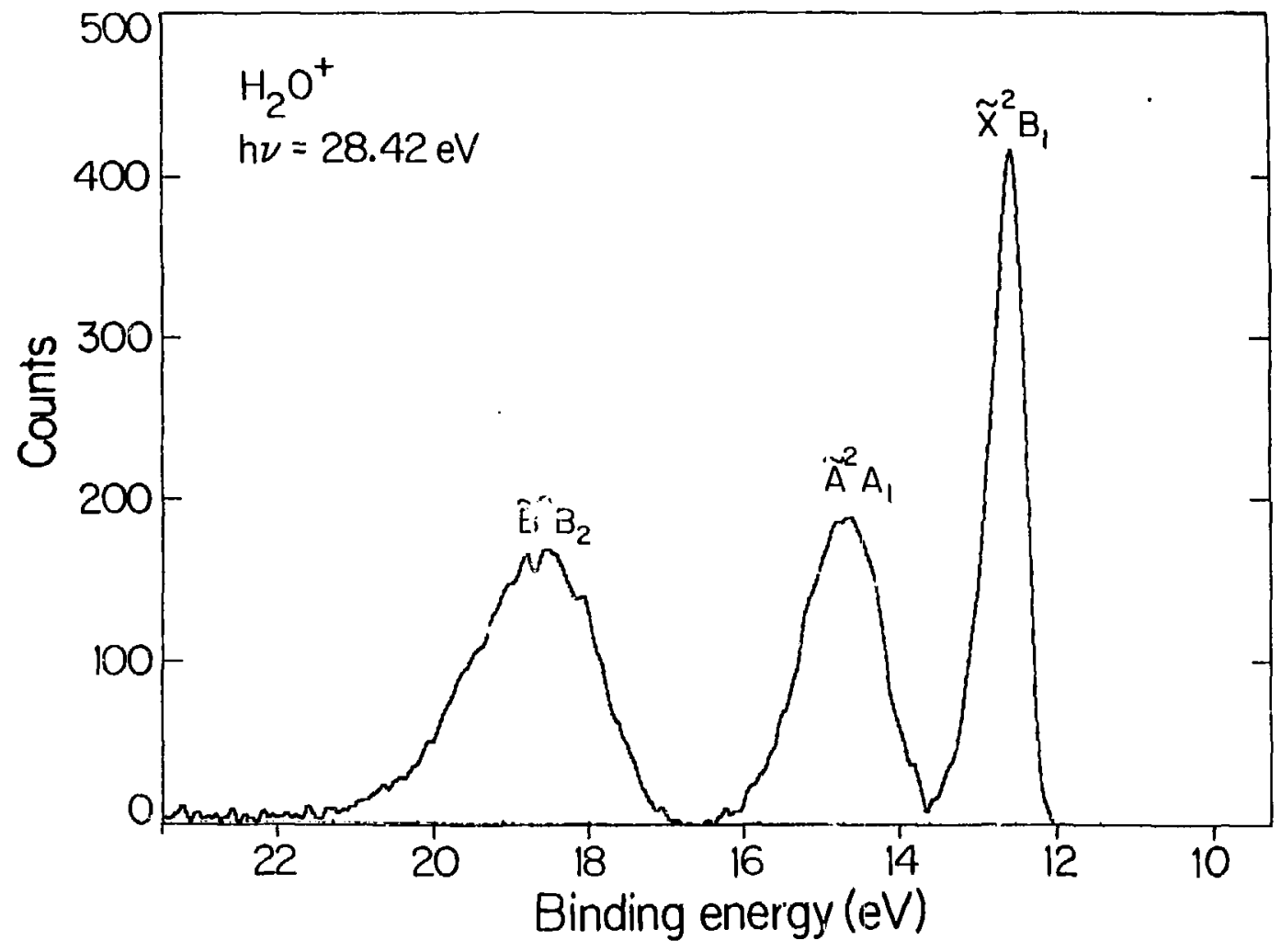

Fig. 2 


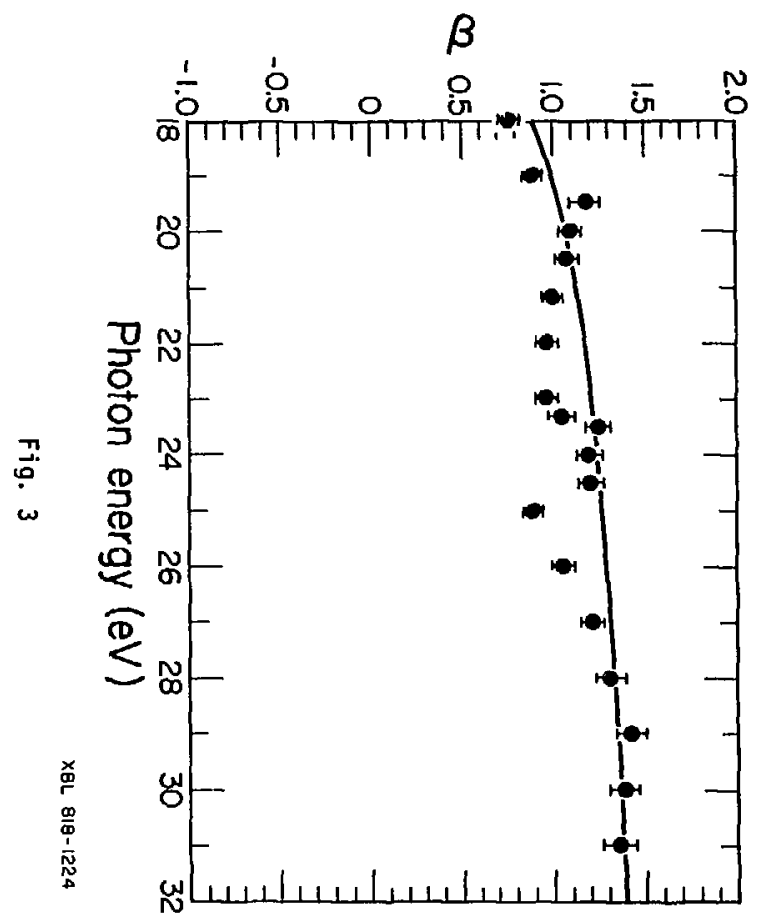

Cross-section (Mb)

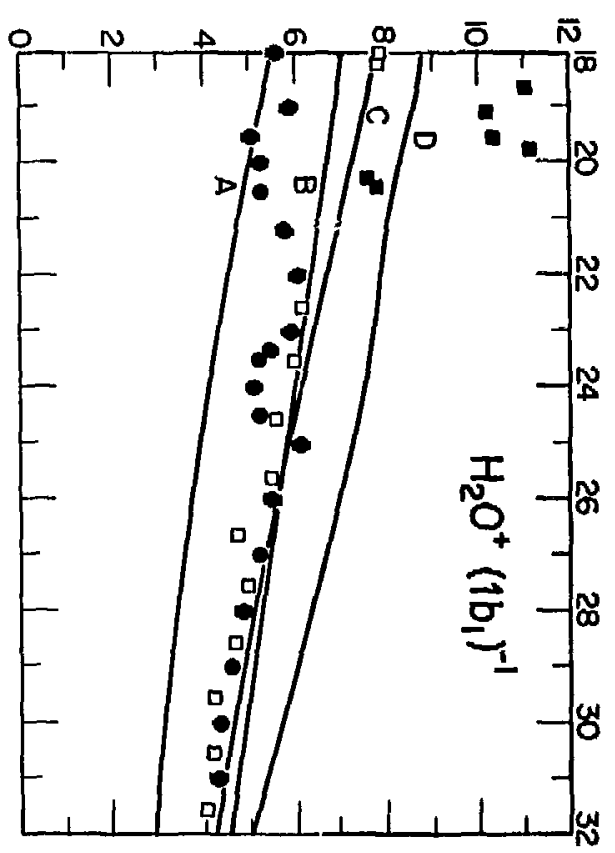



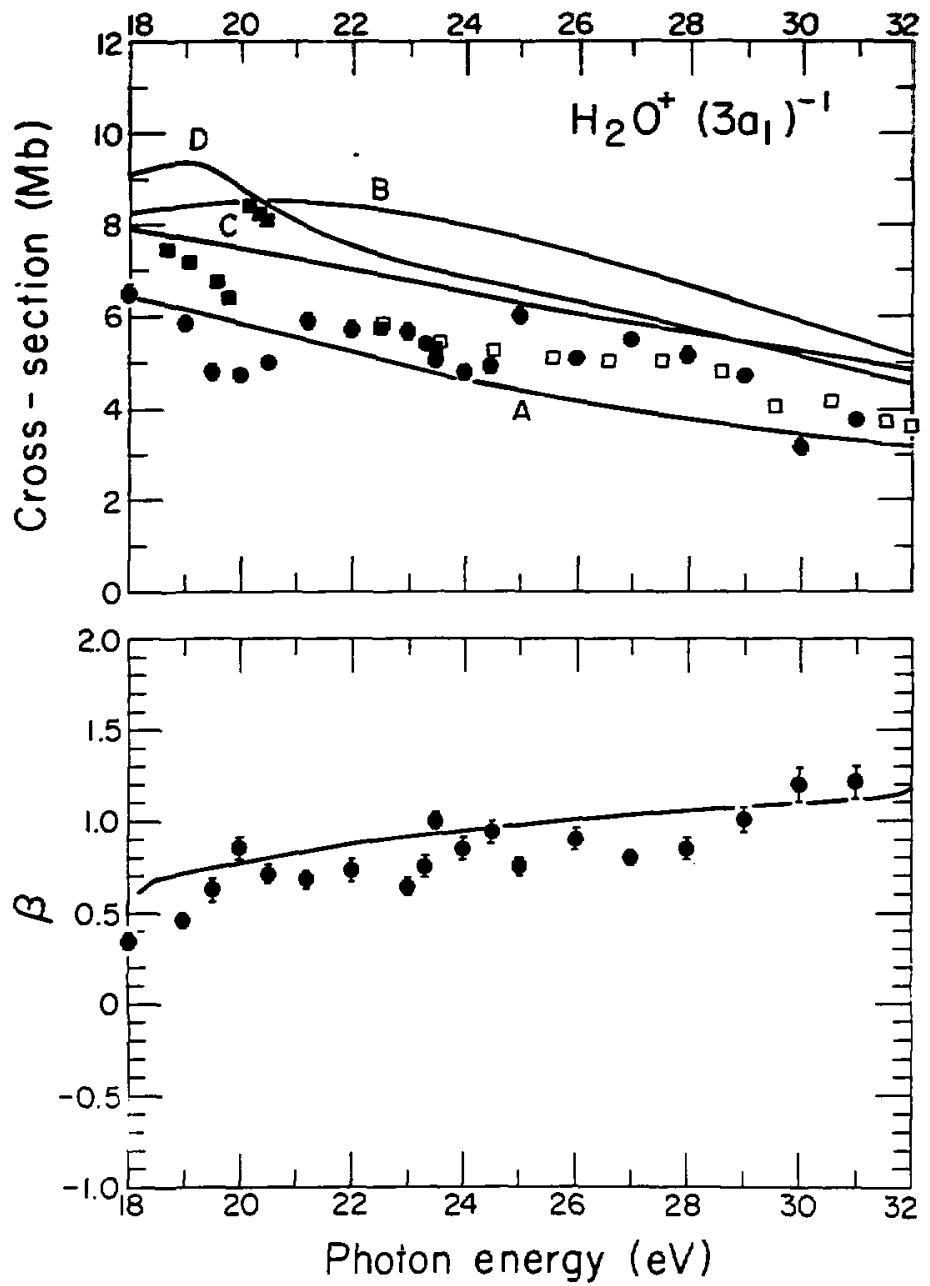

Fig. 3 

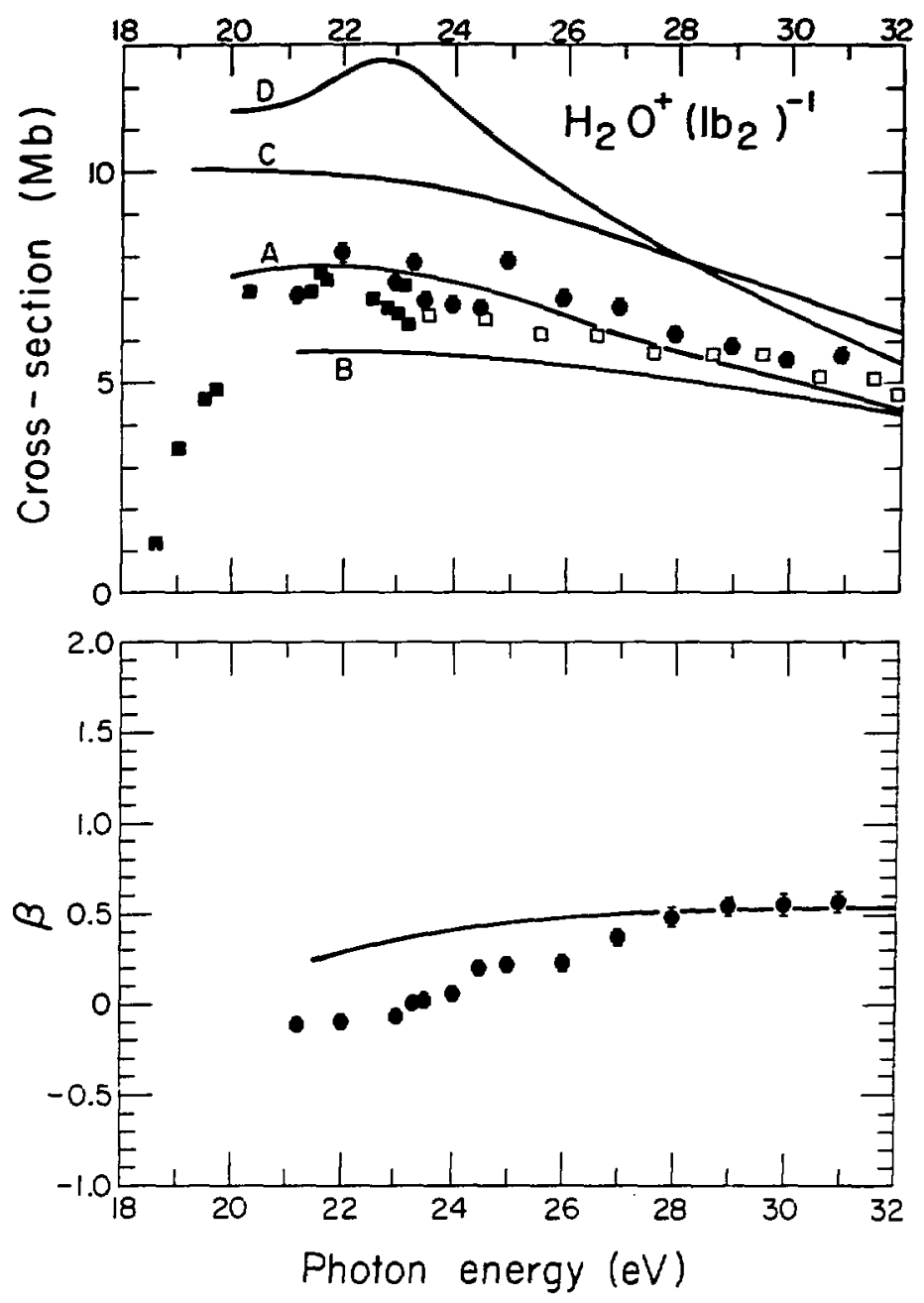

Fig. 4 


\section{PHOTOELECTRON ANGULAR DISTRIBUTIONS OF THE $\mathrm{N}_{2} \mathrm{O}$ OUTER VALENCE ORBitals IN THE 19-31 eV PHOTON EMERgY Ramge*}

\section{A. Introduction}

Photoelectron spectroscopy of the individual molecular orbitals of molecules, using tunable-energy radiation, is in principle capable of yielding a great deal of information about molecular electronic structure. This is a consequence of the fact that the photoionization process is sensitive to dipe? a amplitudes and asymptotic phases, which in turn depend on both the ionization channel and the molecular field.

It is useful first to write the expression for the differential cross section for (dipole) photoemission from a randomly-oriented sample of molecules irradiated by 1 inearly-polarized light: 1

$$
\frac{d \sigma(\varepsilon, \theta)}{d \Omega}=\frac{\sigma(\varepsilon)}{4 \pi}\left[1+B(\varepsilon) P_{2}(\cos \theta)\right] \text {. }
$$

where $P_{2}(\cos \theta)=\left(3 \cos ^{2} \theta-1\right) / 2$ is the second Legendre polynomial, $\theta$ is the angle between the polarization direction and the photoelectron propagation direction, and $\varepsilon$ is the photoelectron kinetic energy. If spin is neglected, ${ }^{2}$ measurement of both $\sigma(\varepsilon)$ and $B(\varepsilon)$ as functions of $c$ will yield all the measurable information about 
a given photoionization channel (e.g., ionization from a given molecular orbital). Variation of $\varepsilon$ is effected by adjusting the photon energy hu from a tunable source, such as synchrotron radiation. The two energies are related by

$$
h u=E+B
$$

for photoionization from an orbital of binding energy 8 . Thus photoelectron spectroscopy can provide more information than that obtained from more conventional methods, such as absorption spectroscopy, because each photoionization channel with a particular binding energy can be monitored independently. In addition, $\sigma(\varepsilon)$ and $B(\varepsilon)$ are sensitive to the molecular potential in different ways. As yet, the development of variable-energy photoelectron spectroscopy is in a very early stage. On the experimental side, the increasing availability and use of synchrotron radiation is facilitating the acquisition of a rapidly growing body of data. For example, recently reported data on molecules such as $\mathrm{H}_{2} \mathrm{O}^{3} \mathrm{OCS},{ }^{4}$ $\mathrm{CS}_{2},{ }^{4} \mathrm{CO}_{2},{ }^{5} \mathrm{~N}_{2},{ }^{6}$ and $\mathrm{O}_{2}{ }^{7}$ have medsured the sensitivity of both $\sigma(\varepsilon)$ and $B(\varepsilon)$ to shape resonances, autoionization, and predissociation, as well as confirming the sensitivity of these parameters to the molecular potential.

The theoretical situation is still evolving. Modelling the photoionization process is a challenge to theory if the goal is to provide a reasonably accurate description of $\sigma(\varepsilon)$ and $s(\varepsilon)$, while 
preserving both computational tractability and a faithful description of physically interesting features in the molecular potentials. Anong the models used to date, the multiple scattering method (MSM) seems todescribe the photoionization process well. Although it assumes a muffin-tin molecular potential and the results are not always quantitative, it has been very successful in describing the energy dependence of $B$ and $\sigma$ for some molecular systems. ${ }^{8}$ Yet to come are a sufficient number of comparisons between theory and experiment to allow broad conclusions to be drawn concerning the efficacy of various theoretical approaches. Still further away is the stage in which quantitative interpretation of the parameters is feasible, without recourse to careful comparison with theory, to obtain quantitatively useful information.

The photoelectron spectra were obtained using a double-angle time-of-flight spectrometer. A detailed discussion of this instrument has been published. 9 Two detectors, one placed at $0^{\circ}$ and another at $54.7^{\circ}$ with respect to the polarization axis of the radiation, detect photoelectrons with microchannel plates. A time spectrum of the photoelectrons is collected, and their intensities as functions of kinetic energy are analyzed to yield $O(\varepsilon)$ and $B(\varepsilon)$. All spectra presented here were collected for 1000 seconds. The $B(\varepsilon)$ parameters are obtained from the intensity ratio of photoelectrons measured at $0^{\circ}$ to those measured at $54.7^{\circ}$. The ratios were corrected for the relative efficiency of the two detectors by a calibration procedure which has been explained in detail. ${ }^{10}$ Relative partial cross 
sections were obtained from the intensity of photoelectrons measured at $54.7^{\circ}$, the "magic angle". At this angle the intensity is independent of the asymmetry parameter. After correcting partial cross sections for the transmission of the $54.7^{*}$ detector, they were normalized for photon flux and sample density, then scaled to the $(e, 2 e)$ total cross sections given by Hitchcock et a1. 11

The Stanford Synchrotron Radiation Laboratory provides a tunable ultraviolet radiation source at the $8^{\circ}$ beam line. Our measurements used photon energies in the range 18-31 eV. The bandpass of the monochromator was $2.7 \AA$ for these experiments. Additional energy resolution broadening from our spectrometer of $-3 \%$ of the kinetic energy of the photoelectrons increases the overall experimental resolution.

This paper is organized in two more sections: B. Results and Discussion, and $C$. Conclusions. The body of the paper appears in Section B, in which photoionization of each valence orbital is discussed in turn.

8. Results and Discussion

The ground state electronic configuration of $\mathrm{N}_{2} \mathrm{O}$ can be written $(1 \sigma)^{2}(2 \sigma)^{2}(3 \sigma)^{2}(4 \sigma)^{2}(5 \sigma)^{2}(6 \sigma)^{2}(1 \pi)^{4}(7 \sigma)^{2}(2 \pi)^{4} \Sigma^{+}$. We present the partial cross sections, branching ratios, and asymmetry parameters for ejection of ohotoelectrons from the $2 \pi, 7 \sigma, 1 \pi$, and 60 orbitals of $\mathrm{N}_{2} \mathrm{O}$, leading respectively to the $X, A, B$, and $C$ states of $\mathrm{N}_{2} \mathrm{O}^{+}$. A complete designation of these channels is given by 


$$
\begin{aligned}
\left.\mathrm{N}_{2} \mathrm{O}^{1} \Sigma^{+}\right)+h_{v} & +\mathrm{N}_{2} \mathrm{O}^{+}\left(x^{2} \overline{\Pi 2 \pi}\right)+\mathrm{e}^{-} \\
& +\mathrm{N}_{2} \mathrm{O}^{+}\left(A^{2} \Sigma+\overline{\Gamma \sigma}\right)+\mathrm{e}^{-} \\
& +\mathrm{N}_{2} \mathrm{O}^{+}\left(\mathrm{B}^{2} \overline{\Pi \pi}\right)+\mathrm{e}^{-} \\
& +\mathrm{N}_{2} \mathrm{O}^{+}\left(\mathrm{C}^{2} \Sigma+\overline{\sigma \sigma}\right)+\mathrm{e}^{-},
\end{aligned}
$$

where $\overline{2 \pi}$ denotes a hole in the $(2 \pi)^{4}$ configuration, etc. A comment on notation is needed. Because the photoionization process involves both initial and final states, we believe that clarity is best served, at the cost of a little redundancy, by fully specifying "the $x^{2} \pi \sqrt{2 \pi}$ channel", etc., throughout.

The mean binding energies of these electronic states are 12.9(X), 15.4(A), 18.3(B), and 20.1(C) eV. A typical spectrum, taken at 24.6 eV photon energy and converted to an energy scale, is shown in fig. 1. The density of data channels varies with the kinetic energy of the detected photoelectrons as $\varepsilon^{-3 / 2}$. Vibrationally-resolved data for the $A^{2} \Sigma^{+} \overline{70}$ and $C^{2} \Sigma^{+} \overline{6 \sigma}$ channels are shown in Figs. 2 and 3 , respectively. It has been proposed by Domcke ${ }^{12}$ that the asymmetry parameter as a function of vibrational state may be used to probe vibronic coupling effects in photoelectron spectra. A simplex a)gorithm ${ }^{13}$ determined the initial estimates for a nonlinear least squares fitting routine, which deconvolute selected spectra. The peak shapes were assumed to be Gaussian. The accuracy of the fitted areas is $10-20 \%$. The variation of the derived partial cross sections and 
asymetry parameters for the vibrational channels is more accurate than an absolute comparison of their values. The assignments of the vibrational modes in $\mathrm{N}_{2} \mathrm{O}^{+}$are given by Dehmer et at. ${ }^{14}$

Our results are discussed in the following format. First, the branching ratios of the ionic states referenced to the total cross section are shown in Fig. 4, along with the total cross section. ...- I contains a tabulation of the partial cross sections and $B(\varepsilon)$ $\therefore t .1$ first four states of $\mathrm{N}_{2} \mathrm{O}^{+}$. There are four subsections to : Section B1 will discuss the $x^{2} \overline{2 \pi}$ channel, Section B2 the $A^{2} \Sigma^{r},-$ channel, Section $B 3$ the $B^{2} \Pi \pi T$ channel, and Section 84 the $c^{2} \Sigma+\overline{60}$ channel.

The other figures fall into two groups. Figs. 7 and 10 show vibrational partial cross sections and asymmetry parameters for the $A$ and $C$ states. They are described in the appropriate subsections. The remaining four figures (5, 6, 8 and 9) display the main results of this work. They all have the same format. The MSM calculations of Whitley and Grimm, which will be discussed in detail in a later publication, ${ }^{15}$ are represented by the solid curves for both $\sigma(\varepsilon)$ and $B(\varepsilon)$. The $(e, 2 e)$ results of $\sigma(\varepsilon)$ from Brion and $\operatorname{Tan}^{16}$ are represented by open circles, and the filled circles represent the present $\mathrm{N}_{2} \mathrm{O}$ measurements. The open triangles in the lower pane 1 of Fig. 5 are our recent $B(\varepsilon)$ results for $\mathrm{CO}_{2}$, which will be described in detail in a later publication.

The $\mathrm{CO}_{2}$ results have been corrected for the difference between the binding eneraies for the $x$ states of $\mathrm{CO}_{2}^{+}$and $\mathrm{N}_{2} \mathrm{O}^{+}$, in order to compare $B(\varepsilon)$ at equal kinetic energies. The motivation frr 
making a comparison between $\mathrm{CO}_{2}$ and $\mathrm{N}_{2} \mathrm{O}$ is that these two molecules are isoelectronic and have similarities in spectral features. The remarkable similarity between the $B(c)$ parameters for the $X$ ionic states of $\mathrm{N}_{2} \mathrm{O}^{+}$and $\mathrm{CO}_{2}^{+}$is intriguing - especially so because the molecules are isoelectronic and the two $x$ states both have ${ }^{2} \pi$ symmetry. The other three states of $\mathrm{N}_{2} \mathrm{O}^{+}$show $\theta(\varepsilon)$ values quite similar to those of the $A, B$, and $C$ states of $\mathrm{CO}_{2}^{+}$. However, we are reluctant to draw conclusions from this observation because states of different symmetry must be paired to produce the best match of $\theta(\varepsilon)$ values.

B1. The $x^{2} \pi \overline{2 \pi}$ Channel

McLean and Yoshimine ${ }^{17}$ have described the $2 \pi$ molecular orbital as a combination of $\mathrm{N}_{1}(2 \mathrm{p})$ and $\mathrm{O}(2 \mathrm{p})$ atomic orbitals. Fig. 5 presents the cross section and as ymmetry parameter for photoelectrons emitted via ionization of this orbital. The partial cross section indicates a probable maximum at $-19.2 \mathrm{eV}$ and a minimum at $21 \mathrm{eV}$, superimposed on a monotonically decreasing overall $\sigma(\varepsilon)$. Dibeler et al ${ }^{18}$ obtained a photoionization curve for $\mathrm{N}_{2} \mathrm{O}$ which shows an autoionizing peak at $19.2 \mathrm{eV}$ that could be due to the population of superexcited, preionized states followed by internal conversion. Eland ${ }^{19}$ observed an (nso) type resonance at $-18.6 \mathrm{eV}$ that appeared strongly in the first four ionic channels.

The MSM calculation agrees only qualitatively with the data in describing the general shape of the decrease in cross section. Our 
cross section measurements are in good overall agreement with the $(e, 2 e)$ results. 16

The $B(c)$ results for the $x^{2} \overline{2 \pi}$ channel, also shown in Fig. 5 , start at a low value of -0.2 at $19 \mathrm{eV}$ and increase to an asymptotic value of -0.8 at $30 \mathrm{eV}$. Because $\sigma(\varepsilon)$ does not show any resonance behavior in the high-energy portion of our measurements, Coulomb phase shifts are presumed to be responsible for the gradual variation in $s(\varepsilon) .20$ The $x^{2} \Pi_{g} \overline{T \pi}_{g}$ channel in $\mathrm{CO}_{2}$ is particularly interesting to consider at this point, because it seems to have an almost identical $B(\varepsilon)$ behavior to that of the $x^{2} \overline{\Pi 2 \pi}$ channel in $\mathrm{N}_{2} \mathrm{O}$ (Fig. 5, lower panel). The $1 \pi_{9}$ molecular orbital in $\mathrm{CO}_{2}$ is composed of out-of-phase overlap of the $p \pi$ atomic orbitals on each of the oxygen atoms. Like the $\mathrm{N}_{2} \mathrm{O} 2 \pi$ orbital, it is nonbonding with a two-fold degeneracy ${ }^{21}$. The similarity in $B(\varepsilon)$ is probably a consequence of the similarity in the ionic potentials. The MSM calculation of $B(\varepsilon)$ for $\mathrm{N}_{2} \mathrm{O}$ agrees qualitatively with the present measurements.

B2. The $A^{2} \Sigma^{+} \overline{70}$ Channel

Calculations suggest that the $7 \sigma$ molecular orbital of $\mathrm{N}_{2} \mathrm{O}$ is composed mainly of $\mathrm{N}_{1}(2 s), \mathrm{N}_{2}(2 p)$, and $O(2 p)$ character, and is weakiy bonding. 17 The partial cross section for the corresponding $A^{2} \Sigma+\frac{\pi}{7 \sigma}$ channel is shown in Fig. 6 . The $\sigma(\varepsilon)$ data again show a maximum at around $19.2 \mathrm{eV}$, and a monotonic decrease to $31 \mathrm{eV}$ photon energy. The maximum in the partial cross section could again be due 
to an autoionizing resonance, as for the $x^{2} \pi \overline{2 \pi}$ channel. There is little agreenent with the MSM calculation. The $(e, 2 e)$ results of Brion and Tan are in excellent agreement with the present measurements, except at $-19.2 \mathrm{eV}$.

The $B(\varepsilon)$ parameter for the $A^{2} \Sigma+\frac{}{7 \sigma}$ channel starts at a low value, near zero, and increases to an asymptotic value of -1.2 . The MSM calculation is in poor agreement with the measured asymetry parameter. The $\mathrm{CO}_{2} A^{2} \Pi T_{\pi_{U}}$ channel $B(\varepsilon)$ parameter (not shown) matches the $\mathrm{N}_{2} \mathrm{O}$ data remarkably well. However, the two channels are very different. The $\pi_{4}$ orbital of $\mathrm{CO}_{2}$ is composed of two perpendicular pairs of $2 p \pi$ orbitals on each atom which are overlapping in-phase to form $\mathrm{C}-0$ bonding. Thus, if the symmetry assignments of the two A states are correct - and they appear to be correct - the similarity of the two $s(\varepsilon)$ parameters remains unexplained.

Fig. 2 shows a typical vibrationally-resolved spectrum collected at $h_{v}=20 \mathrm{eV}$ plotted linearly in time. Both the $\log$ and linear plots were fitted with the simplex and nonlinear least-squares routines. The $\log$ plot confirms that there are five vibrational peaks in the spectrum: in particular, the $(0,0,2)$ line is evident. We could not determine $\sigma(\varepsilon)$ and $g(\varepsilon)$ values for the $(0,0,2)$ peak, because of $i$ ts weah intensity. The four vibrational peaks analyzed correspond to the $(0,0,0),(1,0,0),(0,0,1)$, and the $(1,0,1)$ vibrational modes, with binding energies of $16.40,16.57,16.71$, and $16.85 \mathrm{eV}$, respectively. 22 The quantum numbers represent, from left to right, the symmetric, bending, and asymetric stretch modes. 
The derived $\sigma(\varepsilon)$ and $s(\varepsilon)$ values for the vibrationally resolved $A^{2} \Sigma^{+} \overline{T \sigma}$ channel at selected photon energies are presented in Table II. The $\sigma(\varepsilon)$ and $B(\varepsilon)$ values of the $(0,0,0),(1,0,0),(0,0,1)$, and $(1,0,1)$ channels are shown in $F i g .7$. The $B(c)$ parameter for the $(0,0,1)$ channel is not plotted for clarity, because it has poor statistical error over most of the 19 to $21 \mathrm{eV}$ photon energy range. The $\sigma(\varepsilon)$ and $B(\varepsilon)$ values for individual members of this vibrational manifold show varied behavior, indicating that more information is available at higher resolution. The vibrationally-unresolved cross section, illustrated in Fig. 6 , shows only weak structure in the $h_{v}=$ 19-21 eV region, whereas the resolved cross sections show more variation. The unresolved $B(\varepsilon)$ values tend to smooth out structure because the radial dependencies of the various channels are averaged. ${ }^{23}$ vibrationally-resolved asymmetry parameters could contain information about a whole range of non-Franck-Condon effects, as well as the dependence of $B(\varepsilon)$ on the internuclear separations in molecules. 24 The consequences of these effects on the asymmetry parameter have been discussed theoretically by Itikawa ${ }^{25}$ for non-resonant cases, Dehmer et a $1 .,^{26}$ Stockbauer et al., ${ }^{27}$ and West et al. ${ }^{28}$ have studied the shape-resonance effects, and autoionization effects have been discussed by Jungen et al. ${ }^{29}$

B3. The $B^{2} \pi \sqrt{\pi}$ Channel

According to McLean and Yoshimine, ${ }^{17}$ the $1 \pi$ molecular orbital is mostly the resuit of $\mathrm{N}-\mathrm{O}$ ? bonding with little $\mathrm{N}-\mathrm{N}$ bonding 
character. Our $\sigma(\varepsilon)$ and $B(\varepsilon)$ results are preserictú in Fig. 8 . The partial cross-section data show a low point at $20 \mathrm{eV}$ and a high value at $21.2 \mathrm{eV}$. The low point may arise through a competing mechanism dissociating this channel. Lorquet and Cadet $^{30}$ have calculated potential energy surfaces of $\mathrm{N}_{2} \mathrm{O}$ and a correlation diagram of the $\mathrm{N}_{2} \mathrm{O}$ ion to show the possible dissociation channels. Their calculations prefict an asymptotic dissociation at $20.29 \mathrm{eV}$ above the ground state of $\mathrm{N}_{2} \mathrm{O}$. The process is

$$
N_{2} 0^{+}\left(B^{2} \mathrm{~T}\right)+N_{2}^{+}\left(x^{2} \Sigma_{g}^{+}\right)+0\left({ }^{2} p^{0}\right)
$$

More careful measurements near $20 \mathrm{eV}$ are warranted. The MSM calculation predicts $\sigma(\varepsilon)$ values that are about 50 lower than the present results and that show a different energy dependence. Again, there is good agreement between our data and the $(e, 2 e)$ results.

The $B(\varepsilon)$ values show variations at $-22 \mathrm{eV}$ and below which could arise from the same causes as the variations in $\sigma(\varepsilon)$. The asymmetry parameter $\beta(\varepsilon)$ starts at a low value, near zero, and increases to unity. There is only fair agreement with the MSM calculation of Whitley et al. 15 The $\mathrm{C}^{2} \Sigma_{\mathrm{g}}^{+} \frac{{ }_{\mathrm{H \sigma}}}{\mathrm{g}}$ results for $\mathrm{B}(\varepsilon)$ in $\mathrm{CO}_{2}$ (not shown) are in fair to good agreement with $\beta(\varepsilon)$ for the $B^{2} \pi \overline{1 \pi}$ charnel in $\mathrm{N}_{2} \mathrm{O}$. However, we are again reluctant to draw any conclusions from this similarity because the states have different symmetries. 
84. The $c^{2} \Sigma^{4}-\overline{6 \sigma}$ Channe1

The 60 no:ecular orbital is a combination of $\mathrm{N}-0$ and $\mathrm{N}-\mathrm{N}$ antibonding 5-like orbitals. 17 The partial cross sections and asymmetry parameters for this channel are shown in Fig. 9. The cross section varies slowly over the entire photon energy range, and there is no evidence of resonances. The agreement between the behavior of the calculated MSM partial cross sections and the present results is poor, but once again there is excellent agreement with the $(e, 2 e)$ measurements.

The asymetry parameter for the $c^{2} \Sigma^{+} \overline{60}$ rhannel shows a steep increase from -0.5 at $21 \mathrm{eV}$ to 1.0 at $31 \mathrm{eV}$, with most of the rise occurring between 24 and $27 \mathrm{eV}$. The variation of $B(\varepsilon)$ for this channel is very different than that of the first three channels. As was the case for $\sigma(c)$, there is poor agreement between the MSM calculation of $B(\varepsilon)$ and our results. However, the $B(\varepsilon)$ data for the $B^{2} \Sigma_{u}^{+} \overline{3 \sigma}$ channel in $\mathrm{CO}_{2}$ (not shown) follow these data quite closely. The $3 \sigma_{u}$ molecular orbital of $\mathrm{CO}_{2}$, according to Mulligan, 31 is also nearly nonbonding, with "sp" oxygen character. Hybridized $2 s$ and $2 p$ orbitals on the oxygen atoms over lap a $2 \mathrm{po}$ orbital on the carbon atom.

Fig. 3 shows the partially-resolved vibrational peaks observed in the time-of-flight spectra for the $c^{2} \Sigma^{+} \overline{b \sigma}$ channel. There are three peaks, corresponding to the $(0,0,0),(1,0,0)$, and $(0,0,1)$ channels, with binding energies of $20.15,20.30$, and $20.43 \mathrm{eV}$, respectively. ${ }^{22}$ The $\sigma(\varepsilon)$ and $B(\varepsilon)$ values for these peaks are shown in $\mathrm{Fig.} 10$. 
Partial cross sections and asynmetry parameters are tabulated in Table III. The $s(c)$ results do not show any resonance behavior, but they vary over a large range, and $s(c)$ for the $(1,0,0)$ vibrational mode is near -1 for the entire region measured.

C. Conclusions

The main conclusions of this work are as follows:

1. The partial photoelectron cross sections and asymmetry parameters for the first four ionic states of $\mathrm{N}_{2} \mathrm{O}$ were measured. They were found to be substantially different for the $X, A, B$, and $C$ ionic states, demonstrating the sensitivity of $O(\varepsilon)$ and $B(\varepsilon)$ to details of the different channels sampling the molecular potential.

2. Resolved measurements of $\sigma(\varepsilon)$ and $B(\varepsilon)$ for individual vibrational levels in the $A$ and $C$ states showed large variations with vibrational quantum numbers, demonstrating high sensitivity to non-Franck-Condon effects and/or internuclear spacing.

3. The published $(e, 2 e)$ partial cross sections of Brion and Tan are in good or excellent agreement with the present results. 
4. There is a close empirical, pairwise correspondence between the (c) values for the molecular orbitals of $\mathrm{CO}_{2}$ and $\mathrm{H}_{2} \mathrm{O}$. The correspondence is between the $\mathrm{CO}_{2} x^{2} \pi_{g} T_{\mathrm{g}}$ and $\mathrm{H}_{2} \mathrm{O}$

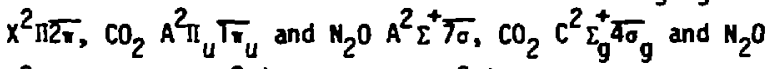
$B^{2} \Pi T \pi$, and $\mathrm{CO}_{2} B^{2} \Sigma_{u}^{+} \overline{3 \sigma}$ and $\mathrm{N}_{2} \mathrm{O} \mathrm{C}^{2} \Sigma^{+} \overline{6 \sigma}$ ionic states. The significance of this observation is unclear. In the first and last pairs, states of the same symmetry are matched, but the other two pairs involve states of opposite symmetry.

5. Better calculations are needed to describe the measured asymmetry parameters and partial cross sections. 


\section{References}

1. C.N. Yang, Phys. Rer 74, 764 (1948).

2. K.N. Huang, Phys. Rev A. 22, 223 (1980).

3. C.M. Truesdale, S. Southworth, P.H. Kobrin, D.W. Lindle, G. Thornton, and D.A. Shirley, J. Chem. Phys, 76, 860 (1982).

4. T.A. Carlson, M.0. Krause, and F.A. Grim, J. Chem. Phys. 77, 1701 (1982).

5. T.A. Carlson, M.O. Krause, F.A. Grimm, J.D. Allen, D. Mehaffy, P.R. Keller, and J.W. Taylor, Phys. Rev. A. 23, 3316 (1981); F.A. Grim, J.D. Allen, Jr., T.A. Carlson, M.O. Krause, D. Mehaffy, P.R. Keller, and J.W. Tayior, J. Chem, Phys. 75, 92 (1981).

6. A.C. Parr, D.L. Ederer, B.E. Cole, J.B. West, R. Stockbauer, K. Codling, S.L. Dehmer, Phys. Rev. Lett. 46, 22 (1981).

7. P. Morin, I. Nenner, M.Y. Adam, M.J. Hubin-Franskin, J. Delwiche, H. Lefebure-Brion, and A. Giusti-Suzor, Chem. Phys. Lett. 92, 609 (1982); P. Morin, I. Nenner, P.M. Guyon, L.F.A. Ferreira, and K. Ito, Chem. Phys. Lett. 92, 103 (1982).

8. M. Roche, D.R. Salahub, and P.R. Messmer, J. Electron Spectrosc. Relat. Phenom., 17, 229 (1979).

9. M.G. White, R.A. Rosenberg, G. Gabor, E.D. Poliakoff, G. Thornton, S.H. Southworth, and D.A. Shirley, Rev. Sci. Instrum. 50, 1288 (1979).

10. S. Southworth, C.M. Truesdale, H.H. Kobrin, D.H. Lindle, W.D. Brewer, and D.A. Shirley, J. Chem. Phys. 76, 143 (1982). 
11. A.P. Hitchcock, C.E. Brion, and M.J. van der Wiel, Chem. Phys. 45, 461 (1980).

12. W. Doncke, Physica Scripta 19, 11 (1979).

13. J.A. Melder and R. Mead, Computer J. 7, 308 (1965); R. O'Neill, Applied Statistics 20, 338 (1971).

14. P.M. Dehmer, J.L. Dehmer, and W.A. Chupka, J. Chem. Phys. 73, 126 (1980).

15. Private communication with T.A. Carlson; T.A. Carlson, P.R. Keller, J.W. Taylor, T.A. Whitley, and F.A. Grimm, to be published.

16. C.E. Brion and K.H. Tan, J. Electron Spectrosc. Relat. Phenom. 15, 241 (1979).

17. A.D. McLean and M. Yoshimine, Tables of Linear Molecular Wave Functions, IBM Corp., San Jose Calif., 1967.

18. V.H. Dibeler, J.A. Walker, and S.K. Liston, J. Res. Nat1. Bur. Stands., 71A, 371 (1967).

19. J.H. Eland, Mol. Phys. 40, 917 (1980).

20. W. Thiel, Chem. Phys. Lett. 87, 249 (1982).

21. A.D. Walsh, J. Chem. Soc. 2266 (1953).

22. C.R. Brundle and D.W. Turner, Int. J. Mass Spectry. Ion Physics 2, 195 (1969).

23. J:R. Swanson, D. Dill, and J.L. Dehmer, J. Phys. B: At. Mol. Phys. 14, L207 (1981).

24. J.W. Rabalais, Principles of UTtraviolet Photelectron Spectroscopy p. 194 Wiley-Interscience, 1977.

25. Y. Itikuwa, Chem. Phys. Lett. 62, 261 (1979); Y. Itikawa, Chem. Phys. 37, 401 (1979). 
26. J.L. Dehmer, D. Dill, and S. Wallace, Phys. Rev. Lett. 43, $1 \operatorname{C05}$ (1979). 27. R. Stockbauer, B.E. Cole, D.L. Ederer, J.B. West, A.C. Parr, and J.L. Dehmer, Phys. Rev. Lett. 43, 757 (1979).

28. J.B. Hest, A.C. Parr, B.E. Cole, D.L. Ederer, R. Stockbauer, and J.L. Dehmer, J. Phys B 13, L105 (1980).

29. Ch. Jungen and O. Altebek, J. Chem. Phys. 66, 5584 (1977); Ch. Jungen and D. Dill, J. Chem. Phys. 13,3338 (1980).

30. J. C. Lorquet and C. Cadet, Int. J. Mass Spectry. Ion Physics ?, 245 (1971).

31. J.F. Mulligan, J. Chem. Phys. 32, 1595 (1951).

32. T.A. Carlson and G.E. Mc Guire, J. Electron Spectrosc. Relat. Phenom. 1, 209 (1972). 
Table 1. Cross sections and asymetry parameters of the $x_{1} A, B$, and $C$ Channels of $\mathrm{H}_{2} \mathrm{O}^{+}$.

\begin{tabular}{|c|c|c|c|c|c|c|c|c|}
\hline \multirow[b]{2}{*}{$h v(e V)$} & \multicolumn{2}{|c|}{$x^{2} \pi \overline{2}$} & \multicolumn{2}{|c|}{$A^{2} \vec{E} \overline{7_{0}}$} & \multicolumn{2}{|c|}{ 8रिपा1 } & \multicolumn{2}{|c|}{$c^{2} \Sigma^{4} \overline{6 \sigma}$} \\
\hline & $A(M B)^{\prime \prime}$ & $s(x)$ & $O(+40)$ & $1(x)$ & $a\left(K_{b}\right)$ & $0(e)$ & o(e) & $1(e)$ \\
\hline 19.0 & $15.29(34)^{b}$ & $0.24(2)$ & $12.09(28)$ & $0.07(1)$ & & & & \\
\hline 19.2 & $17.00(40)$ & $-0.15(3)$ & $15.57(37)$ & $0.00(3)$ & $8.89(22)$ & $0.20(4)$ & & \\
\hline 20.0 & $15.64(35)$ & $-0.12(2)$ & $10.90(25)$ & $0.20(3)$ & $7.89(18)$ & $0.22(3)$ & & \\
\hline 20.5 & $14.30(32)$ & $-0.06(3)$ & $9.12(21)$ & $0.39(4)$ & $10.17(23)$ & $0.18(3)$ & & \\
\hline 21.2 & $12.27(27)$ & $\begin{array}{r}-0.03(3) \\
0.0(1)^{c}\end{array}$ & $8.76(21)$ & $\begin{array}{l}0.43(1) \\
0.35(10)^{c}\end{array}$ & $11.48(27)$ & $\begin{array}{r}0.12(3) \\
-0.2(2)^{c}\end{array}$ & $6.70(16)$ & $\begin{array}{l}-0.36(2) \\
-0.4(2)^{c}\end{array}$ \\
\hline 21.8 & $13.03(30)$ & $0.15(3)$ & $6.30(15)$ & $0.67(5)$ & $10.26(25)$ & $0.48(4)$ & $5.94(14)$ & $-0.26(2)$ \\
\hline 23.0 & $14.18(31)$ & $0.32(3)$ & $6.34(15)$ & $0.81(2)$ & $10.28(24)$ & $0.45(4)$ & $6.24(12)$ & $-0.17(2)$ \\
\hline 24.0 & $13.12(29)$ & $0.41(4)$ & $5.55(13)$ & $0.90(3)$ & $9.93(23)$ & $0.58(4)$ & $5.70(14)$ & $-0.13(3)$ \\
\hline 25.0 & $13.74(30)$ & $0.50(4)$ & $4.59(12)$ & $1.00(3)$ & $10.01(2.4)$ & $0.73(5)$ & $5.39(13)$ & $0.13(3)$ \\
\hline 26.0 & $12.52(28)$ & $0.61(4)$ & $4.27(11)$ & $1.06(3)$ & $9.31(22)$ & $0.85(5)$ & $4.61(12)$ & $0.45(5)$ \\
\hline 22.0 & $12.70(31)$ & $0.68(5)$ & $4.38(13)$ & $1.07(6)$ & $8,49(22)$ & $1.08(7)$ & $4.58(13)$ & $0.60(7)$ \\
\hline 28.0 & $12.29(22)$ & $0.86(5)$ & $4.23(10)$ & $1.15(7)$ & $8.46(19)$ & $1.00(6)$ & $3.67(9)$ & $0.87(6)$ \\
\hline 29.0 & $11.27(29)$ & $0.76(5)$ & $4.88(15)$ & $1.03(4)$ & $8.56(2 ?)$ & $1.06(7)$ & $3.13(10)$ & $0.90(7)$ \\
\hline 29.5 & $8.80(22)$ & $0.88(6)$ & $3.83(11)$ & $1.17(8)$ & $7.61(19)$ & $1.01(6)$ & $3.04(12)$ & $0.95(1)$ \\
\hline 31.0 & $9.87(31)$ & $0.78(6)$ & $4.37(18)$ & $1.16(5)$ & $7.92(25)$ & $1.09(9)$ & $3.26(15)$ & $0.96(10)$ \\
\hline
\end{tabular}

(a) Absolute cross sections were obtained by sraling to (e,2e) data (Ref. II).

(b) Errors in the last place are given parenthetically.

(c) Ref. 32 . 
Table 11. Cross sections and asymmetry parameters of the $\mathrm{A}^{2} 2_{2}^{+} \overline{\%}_{0}$ vibrational channels of $\mathrm{N}_{2} \mathrm{O}^{+}$.

\begin{tabular}{|c|c|c|c|c|c|c|c|c|}
\hline \multirow[b]{2}{*}{$h_{u}(\mathrm{eV})$} & \multicolumn{2}{|c|}{$(0,0,0)$} & \multicolumn{2}{|c|}{$(1,0,0)$} & \multicolumn{2}{|c|}{$(0,0,1)$} & \multicolumn{2}{|c|}{$(1,0,1)$} \\
\hline & $\sigma(M b)^{a}$ & $B(\varepsilon)$ & $\sigma(M b)$ & $B(\varepsilon)$ & $\sigma(M b)$ & $B(\varepsilon)$ & $\sigma(M b)$ & $B(c)$ \\
\hline 19.0 & $7.42(16)$ & $0.16(3)$ & $3.39(16)$ & $0.04(5)$ & $0.72(7)$ & $0.76(9)$ & $0.55(13)$ & $-.45(7)$ \\
\hline 19.2 & $9.33(34)$ & $0.11(3)$ & $4.91(35)$ & $-0.34(5)$ & $0.94(19)$ & $0.70(25)$ & $0.39(5)$ & $.02(12)$ \\
\hline 20.0 & $6.12(47)$ & $0.36(2)$ & $3.46(47)$ & $-0.30(10)$ & $1.09(40)$ & $0.23(45)$ & $0.21(2)$ & $.28(16)$ \\
\hline 20.5 & $5.37(38)$ & $0.76(11)$ & $3.61(40)$ & $-0.21(8)$ & $0.52(10)$ & $0.90(36)$ & $0.42(11)$ & $.18(36)$ \\
\hline 21.2 & $5.86(12)$ & $\begin{array}{l}0.57(3) \\
0.35^{b}\end{array}$ & $1.78(8)$ & $\begin{array}{l}0.61(9) \\
0.45(5)^{b}\end{array}$ & $0.76(4)$ & $\begin{array}{l}0.49(15) \\
0.20(20)^{b}\end{array}$ & $0.35(4)$ & $.02(26)$ \\
\hline
\end{tabular}

(a) The total cross section was obtained by scaling to $(e, 2 e)$ data (Ref. 11). (b) Ref. 32 . 
Table III. Cross sections and asymmetry parameters of the $c^{2} \Sigma^{+} \overline{60}$ vibrational channels of $\mathrm{N}_{2} \mathrm{O}^{+}$.

\begin{tabular}{|c|c|c|c|c|c|c|}
\hline \multirow[b]{2}{*}{$h_{v}(e v)$} & \multicolumn{2}{|c|}{$(0,0,0)$} & \multicolumn{2}{|c|}{$(1,0,0)$} & \multicolumn{2}{|c|}{$(0,0,1)$} \\
\hline & $\sigma(M b)^{a}$ & $B(\varepsilon)$ & $\sigma(M b)$ & $B(c)$ & $\sigma(M b)$ & $B(c)$ \\
\hline 23.0 & $4.05(10)$ & $.36(3)$ & $1.32(7)$ & $-.75(4)$ & $0.88(7)$ & $-.51(6)$ \\
\hline 24.0 & $3.53(38)$ & $.15(11)$ & $1.17(24)$ & $-.72(6)$ & $1.38(17)$ & $-.46(9)$ \\
\hline 25.0 & $3.45(38)$ & $.57(12)$ & $1.65(70)$ & $-.70(13)$ & $1.35(23)$ & $-.18(12)$ \\
\hline 26.0 & $3.14(23)$ & $.80(5)$ & & & $1.47(18)$ & $-.17(11)$ \\
\hline
\end{tabular}

(a) The total cross section was obtained by scaling to $(e, 2 e)$ data (Ref. 11). 


\section{Figure Captions}

Figure 1. TOF photoelectron spectrum showing the $x^{2} \overline{2 \pi}, A^{2} \Sigma^{+} \overline{7_{\sigma}}$, $B^{2} \overline{M i}$, and $C^{2} \Sigma^{+} \overline{6 \sigma}$ ionic states of $\mathrm{N}_{2} \mathrm{O}^{+}$.

Figure 2. TOF photoelectron spectrum showing the $A^{2} \Sigma^{+} \overline{7 \sigma}$ vibrational states of $\mathrm{N}_{2} \mathrm{O}^{+}$. The curves in the linear spectrum are deconvoluted peaks from nonlinear leastsquares fits. The curve in the $\log$ plot represents the sum of deconvoluted peaks calculated with the same fitting method.

Figure 3. TOF photoelectron spectrum showing the $c^{2} \Sigma^{+}+\frac{}{6 \sigma}$ vibrational states of $\mathrm{N}_{2} \mathrm{O}$. The curves are the deconvoluted peaks as in Fig. 2.

Figure 4. Branching ratios to the ionic states in $\mathrm{N}_{2} \mathrm{O}^{+}$, and total photoionization cross section of $\mathrm{N}_{2} \mathrm{O}$. The symbols represent states as follows: $X(\Delta), A(O), B(\square)$, and C(D). All are referenced to our total cross-section measurements, denoted by $\boldsymbol{O}$. The $\Delta$ are $(e, 2 e)$ total cross-section measurements of Brion and Tan. 16 
Figure 5. The $\sigma(c)$ and $B(c)$ results for the $x^{2} \| \overline{2}$ ionic state. The present results are plotted as filled circles. The curves are ine MSM calculations of Whitley and Grimm. 15 Open circles in the $\sigma(\varepsilon)$ plot are the $(e, 2 e)$ results of Brion and Tan. ${ }^{16}$ Open triangles in the $B(\varepsilon)$ pane $l$ are our measurements on the $x^{2} \pi_{g} \overline{1 \pi}_{g}$ state of $\mathrm{CO}_{2}$. The $\mathrm{CO}_{2}$ results were adjusted by 0.9 eV to compare equal kinetic energies.

Figure 6. The $\sigma(\varepsilon)$ and $B(\varepsilon)$ results for the $A^{2} \Sigma^{+} \overline{7 \sigma}$ ionic state. The notation is similar to Fig. 5 .

Figure 7. Vibrational resolved $\sigma(\varepsilon)$ and $\theta(\varepsilon)$ of the $A^{2} \Sigma^{+} \overline{\eta_{\sigma}}$ ionic state.

Figur. 8. The $\sigma(\varepsilon)$ and $\theta(\varepsilon)$ values for the $\theta^{2} \pi \pi$ ionic state. The notation is similar to Fig. 5 .

Figure 9. The $\sigma(\varepsilon)$ and $\beta(\varepsilon)$ values for the $C^{2} \Sigma^{+} \overline{6 \sigma}$ ionic state. The notation is similar to Fig. 5 .

Figure 10. Vibrational recolved $\sigma(\varepsilon)$ and $g(\varepsilon)$ of the $c^{2} \Sigma^{+} \overline{6 \sigma}$ ionic state. 


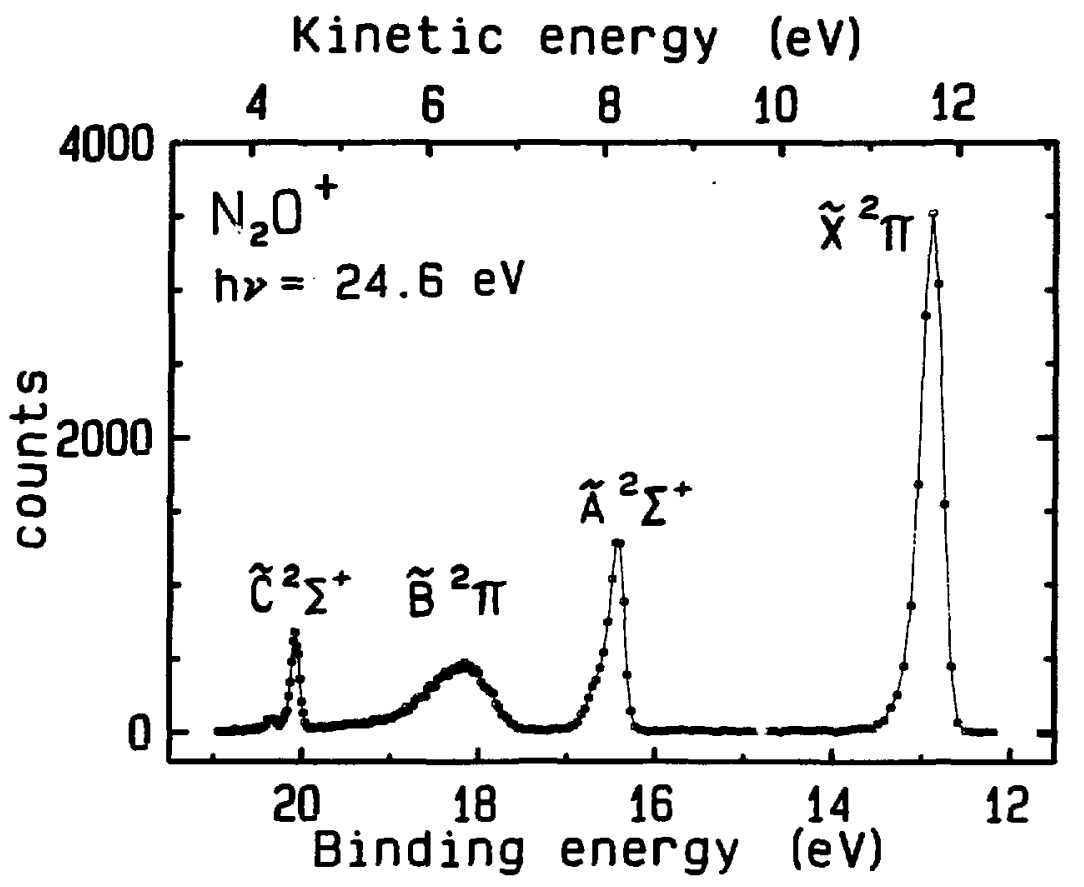

Fig. 1 


\section{4}

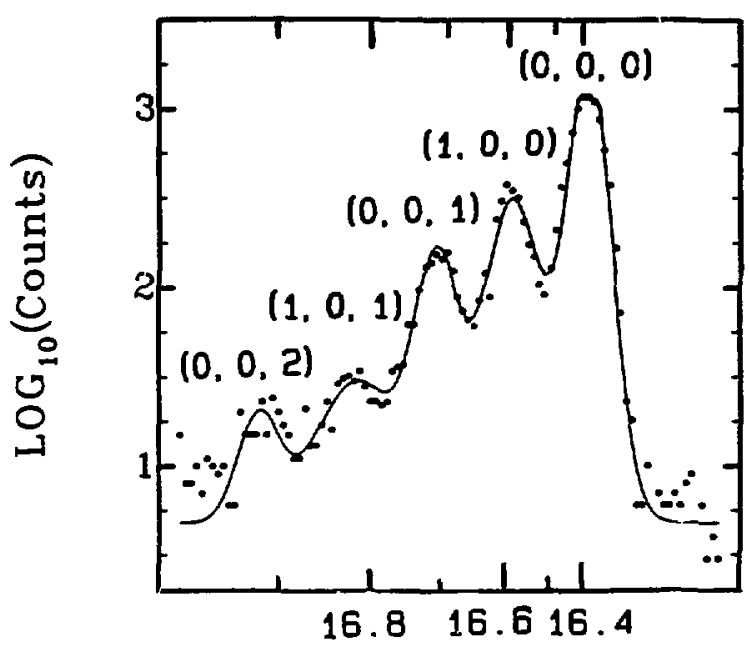

Binding energy $(\in V)$

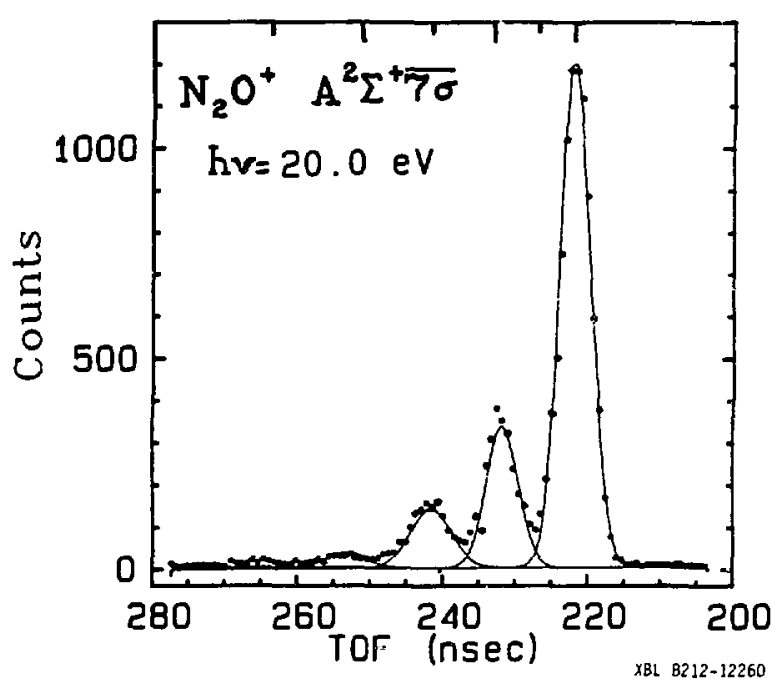

Fig. 2 


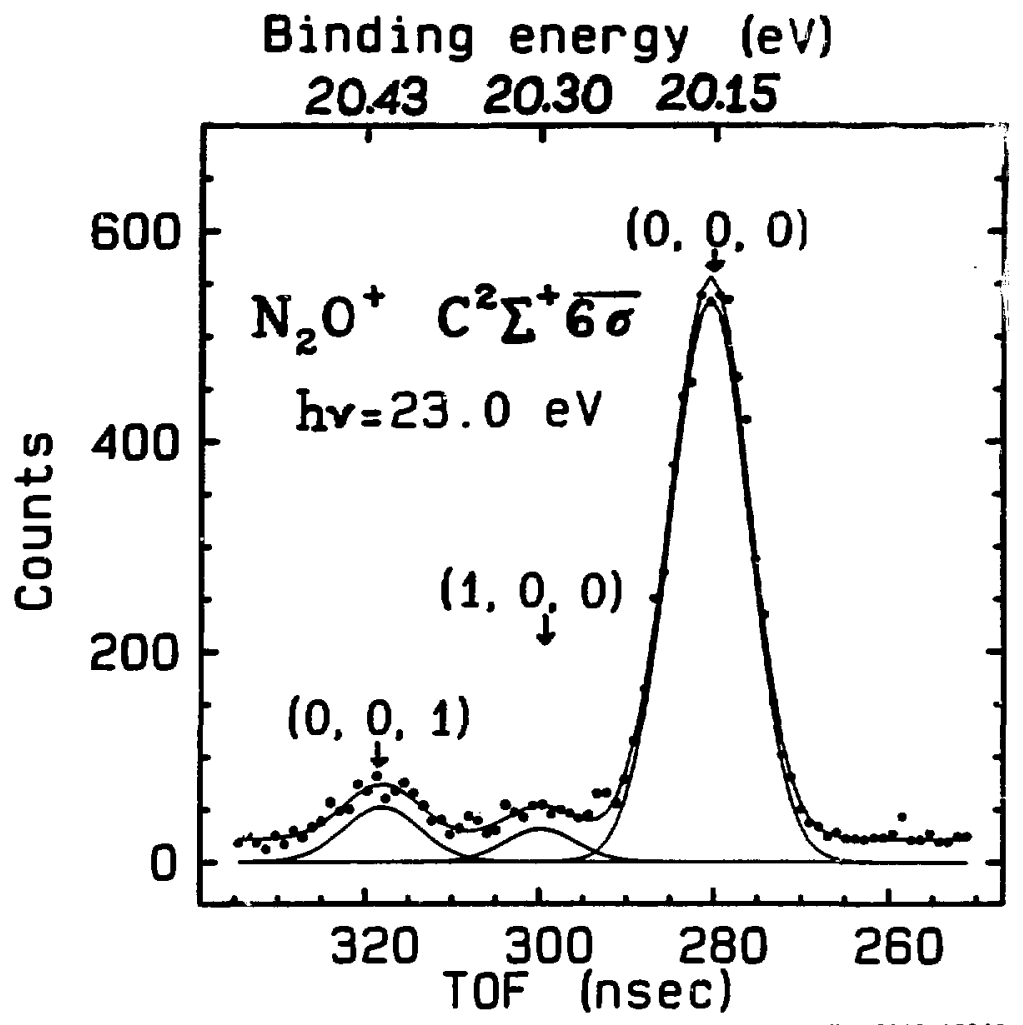

Fig. 3 


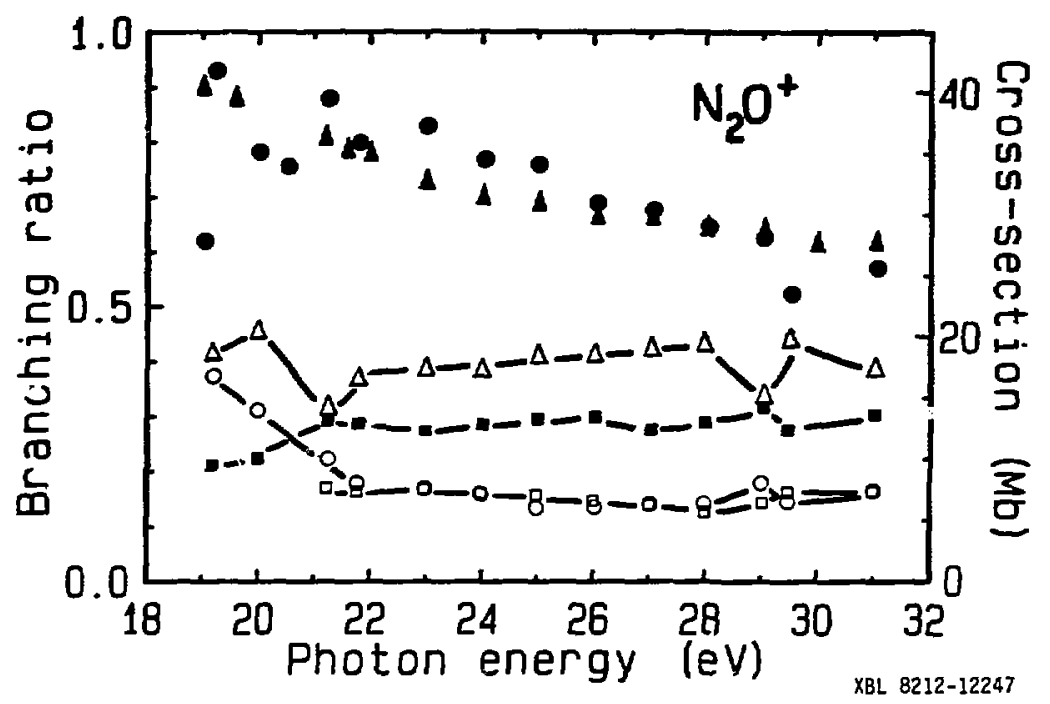

Fig. 4 

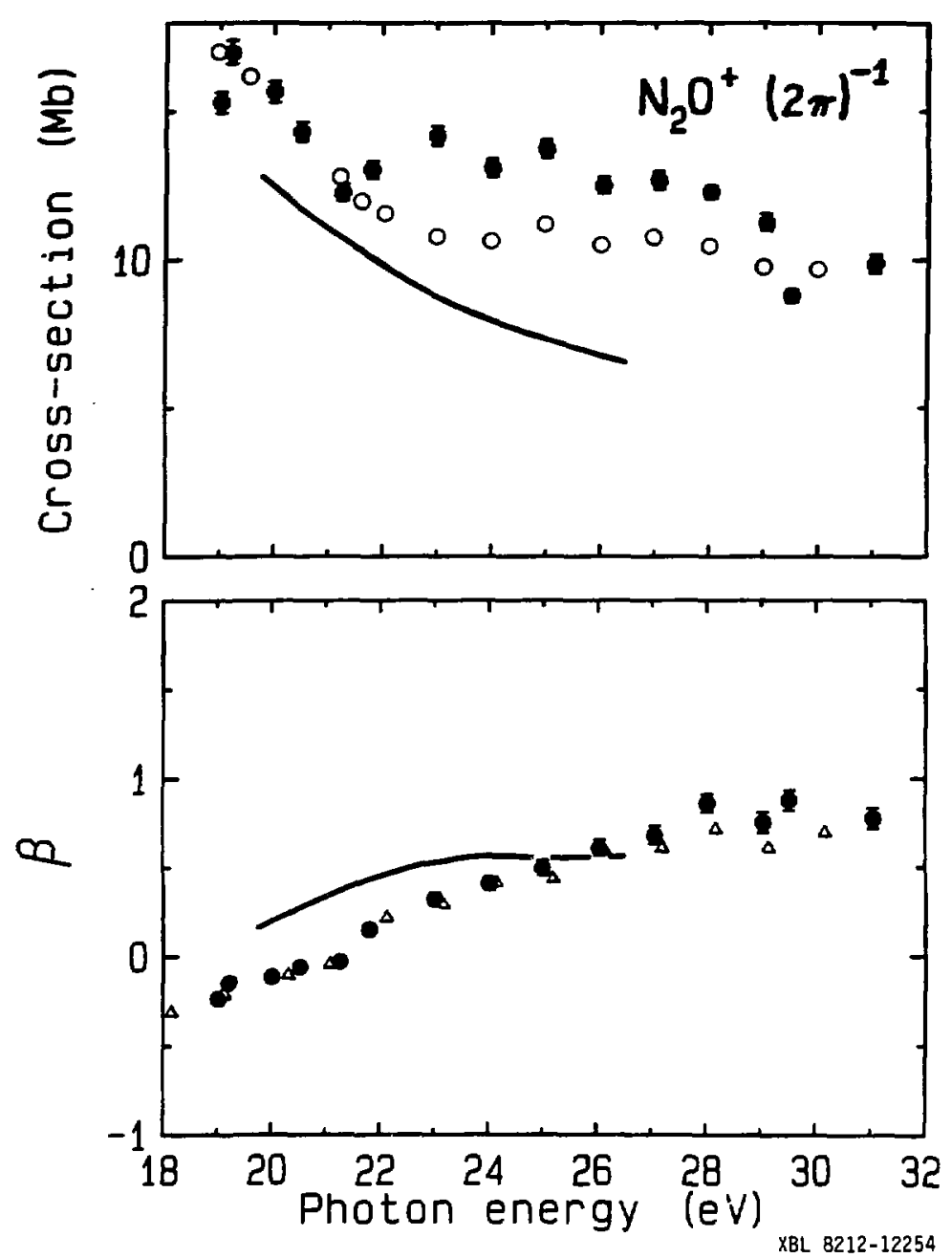

Fig. 5 

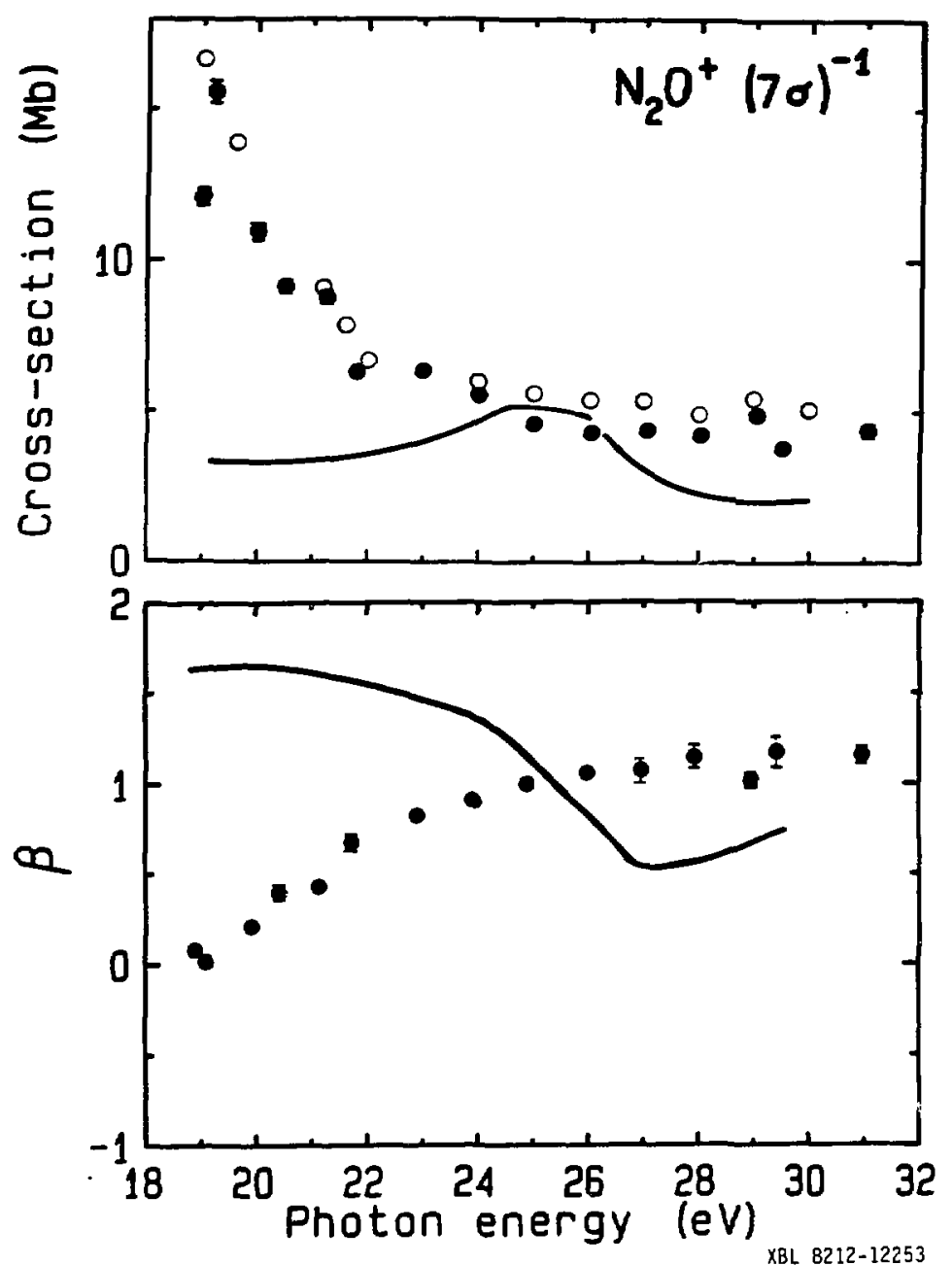

Fig. 6 

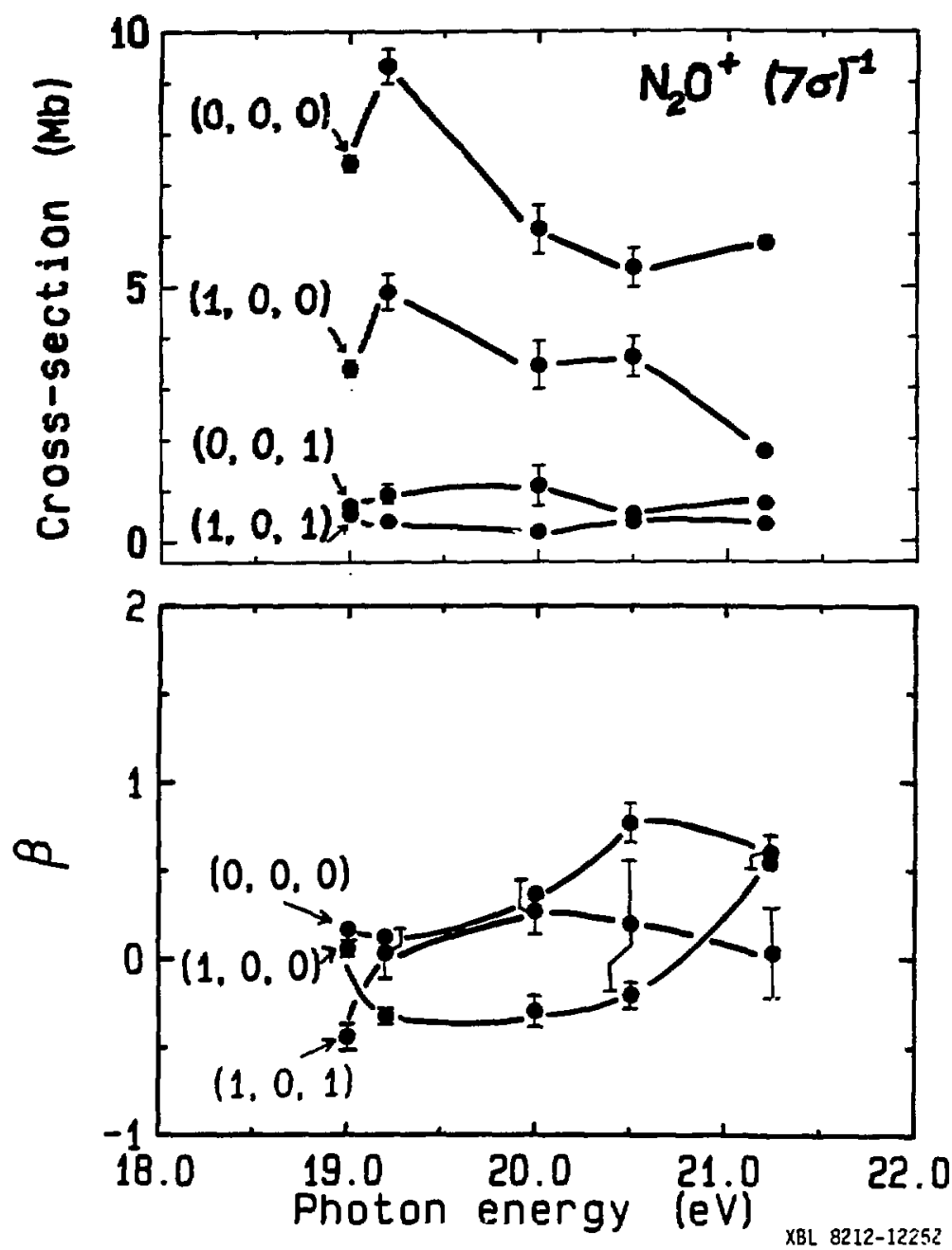

Fig. 7 


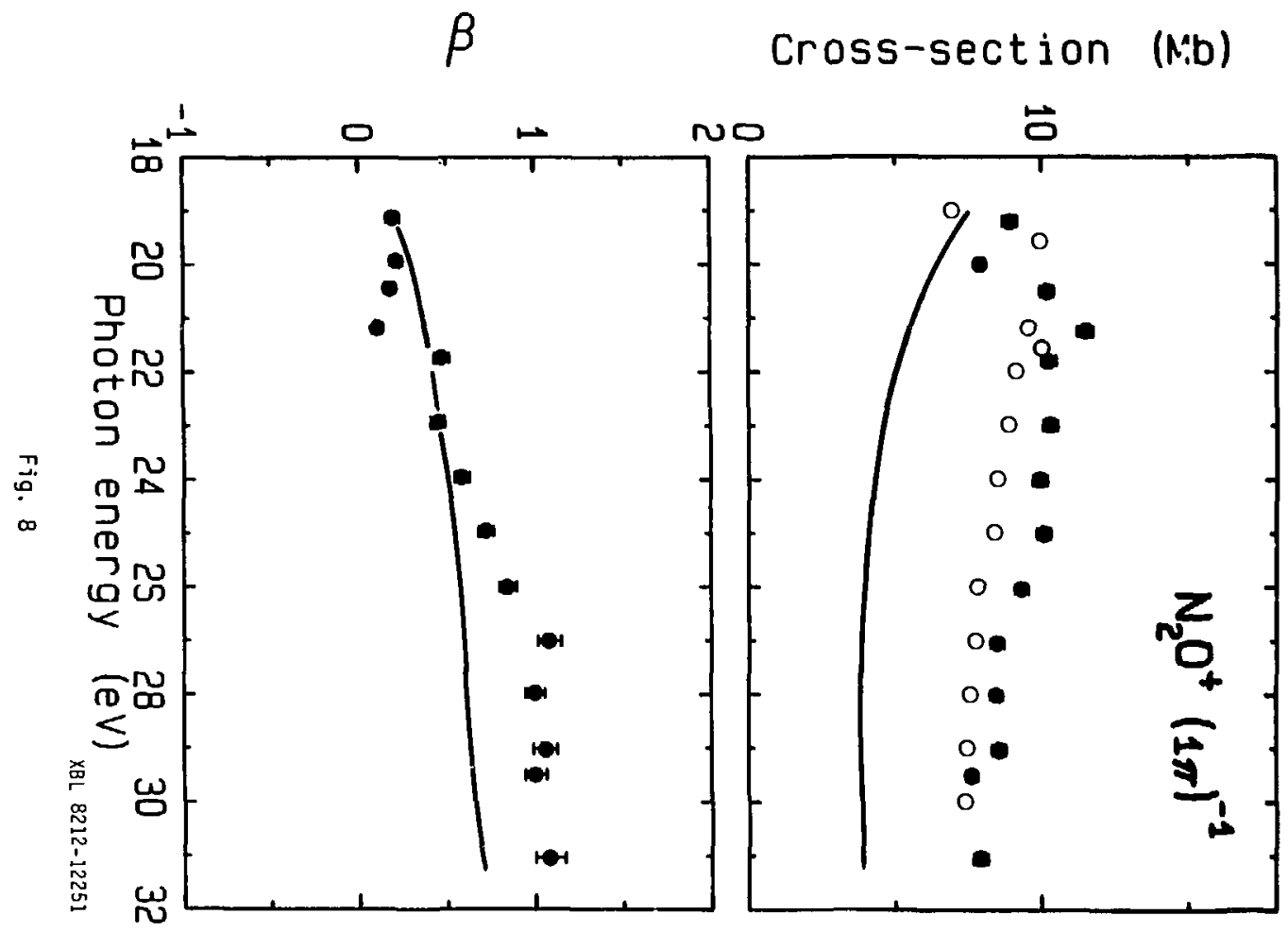

\& 


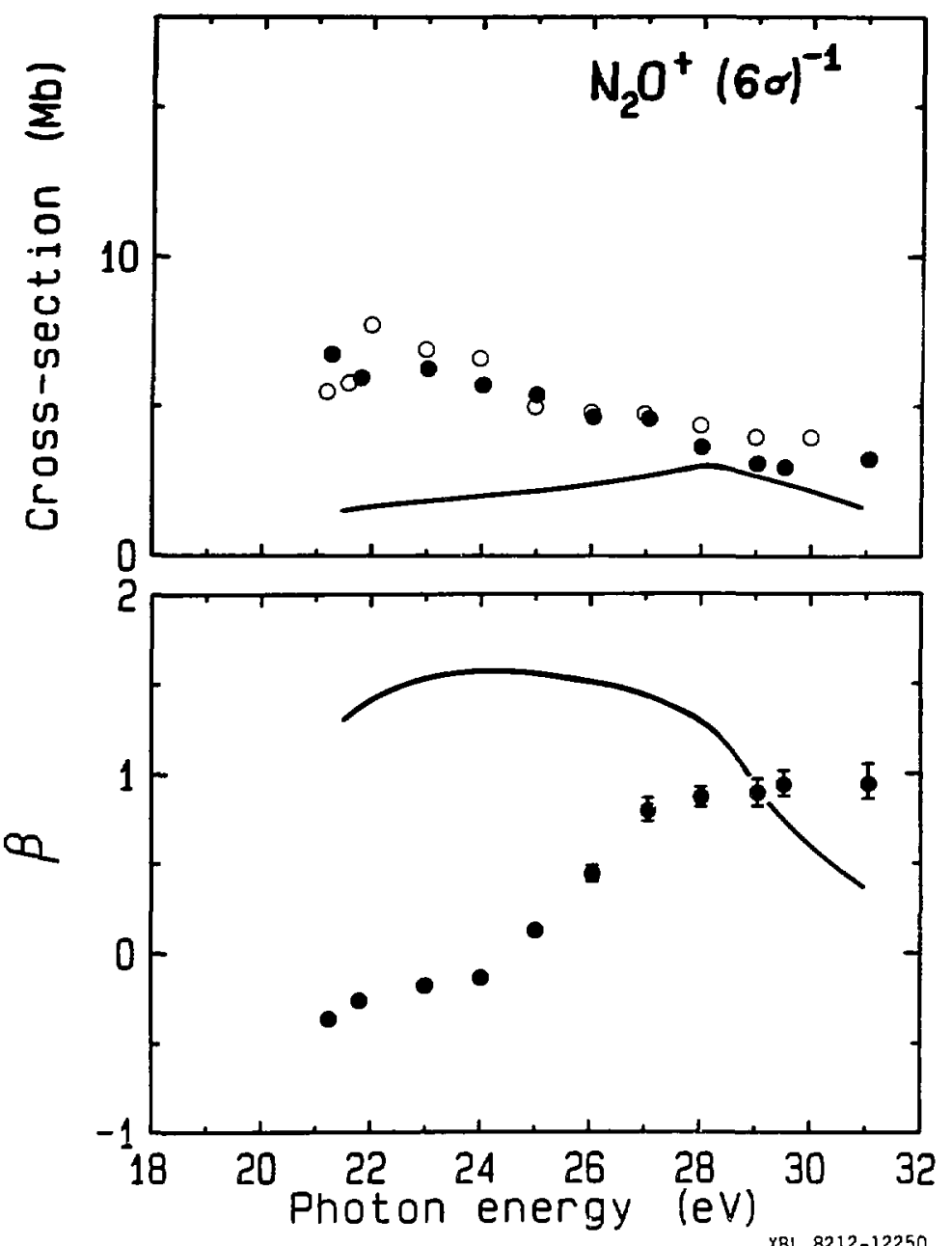

Fig. 9 
82
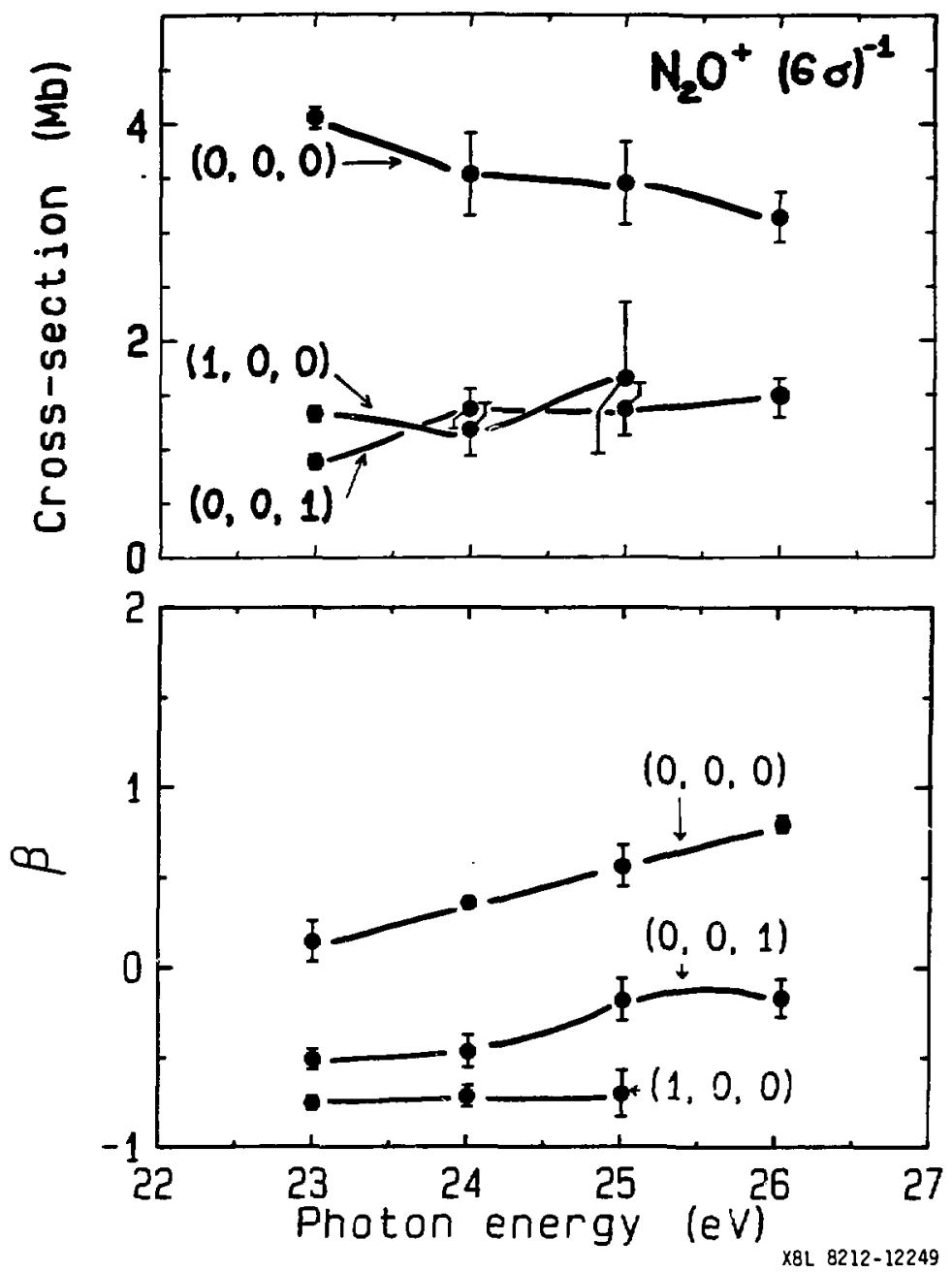

Fig. 10 


\section{Y. VIBRATIONALLY ANGLE-RESOLVED PHOTOELECTRON STUDIES OF $\mathrm{CO}_{2}$ AND OCS}

\section{A. Introduction}

Studies of the vibrational levels via photoionization can provide information about internuclear-distance dependent structure ${ }^{1}$ and nonFranck-Condon behavior ${ }^{1-4}$ for the vibrational intensity distribution and photoelectron asymmetry parameters. The asymmetry parameter $\theta(\varepsilon)$, which describes the angular distribution of photoelectrons, is related to the differential cross section do/d $\Omega$, by ${ }^{5}$

$$
\frac{d \sigma(\varepsilon, \theta)}{d \Omega}=\frac{\sigma(\varepsilon)}{4 \pi}\left[1+B(\varepsilon) P_{2}(\cos \theta)\right] \text {. }
$$

Here $P_{2}(\cos \theta)$ is the second Legendre polynomial $\left[\left(3 \cos ^{2} \theta-1\right) / 2\right]$ and $\theta$ is the angle of photcelectron emission referenced to the photon beam polarization direction. The total cross section $\alpha\{\varepsilon\}$ and the asymetry parameter $B(\varepsilon)$ are functions of the photoelectron kinetic energy $\varepsilon$. Two time-of-flight (TOF) detectors, placed at $\theta=0^{\circ}$ and $54.7^{\circ}$, can be used to analyze ejected photoelectrons. From equation (1), measurements at these two angles suffice to determine o $\{\varepsilon\}$ and $B(\varepsilon)$.

* C.M. Truesdale, S. Southworth, P.H. Kobrin, U. Becker, D.W. Lindle, and C.A. Shirley, submitted to Phys. Rev. A (1983). 
The vibrational branching ratios $\left(R_{n}\right)$ are defined by

$$
R_{n}=\frac{\sigma\left(v_{n}\right)}{\sigma\left(v_{0}\right)},
$$

where $\sigma\left(v_{n}\right)$ is the photoionization cross section of the $v_{n}$ th vibrational channel and $\sigma\left(v_{0}\right)$ is the cross section of the ground vibrational channel $(0,0,0)$. For the $A^{2} \pi_{U}$ ionic channel of $\mathrm{CO}_{2}$, $v_{n}$ will refer to the symmetric stretch vibrational channels $(n, 0,0)$. The first quantum number $n$ designates the symmetric stretch modes, where $n=0,1,2,3$, and 4 . The other quantum numbers refer to the bending and asymetric stretch vibrational modes. During TOF measurements, all photoelectrons are simultaneously measured. The vibrational branching ratios are inherently more accurate than absolute cross sections, because normalization of photoelectron intensities for sample density and photon flux are not needed.

The purpose of this paper is to report vibrationally-averaged photoionization cross sections and photoelectron asymmetrv paramet ars for the $1 \pi_{u}$ and 80 orbitals of $\mathrm{CO}_{2}$ and OCS, which lead to the ionic $A^{2} \eta_{u}$ and $C^{2} \Sigma^{+}$states, respectively. The vibrationally-resolved branching ratios and asymetry parameters for these ionic channels are also reported. These data are compared to other sy:ichrotron results, ${ }^{6-10}$ dipole $(e, 2 e)$ results, ${ }^{11}$ and He I resonance light source results. ${ }^{12-18}$ Multiple-scattering model (MSM) calculations $1,6,7,12$ for $\mathrm{CO}_{2}$ and oCs, and Hartree-Fock static-exchange (HF) calculations ${ }^{19}$ and StieltjesTchebycheff moment theory (STMT) results ${ }^{20}$ for $\mathrm{CO}_{2}$ are compared to the exoerimental results. 
The photoelectron spectra were obtained with a double-angle-timeof-flight spectrometer. Two detectors, one placed at $0^{\circ}$ and enother placed at $54.7^{*}$ with respect to the photon polarization axis, were used to measure ejected photoelectrons by means of microchannel plates. The instrument was described previously. ${ }^{21}$ The experiments were performed at the Stanford Synchrotron Radiation Laboratory, on the VUV $\left(8^{\circ}\right)$ team line with a monochromator resolution of $2.7 \mathrm{~A}$ (FWHM) throughout the entire photon energy range hv $=18-30 \mathrm{eV}$. The spectrometer kinetic energy resolution $\Delta \varepsilon$ is 3 percent of $\varepsilon$. By retarding the photoelectrons over part of the $28 \mathrm{~cm}$ flight path the resolution could be improved significantly.

The intensity measurements at $54.7^{\circ}$, for which $P_{2}=0$, provide angle-independent relative cross sections. The counting period was typically 1000 seconds for the vibrationally-averaged studies of $\mathrm{CO}_{2}$ and OCS and the vibrationally-resolved studies of $\mathrm{CO}_{2}$ and 2000 seconds for the OCS vibrationally resolved measurements. The total relative cross section for the photoionization of the four outer valence orbitals of $\mathrm{CO}_{2}$ and oCS was compared to the dipole $(e, 2 e)$ total cross sections given by Hitchcock et al., ${ }^{22}$ and white et al., ${ }^{23}$ respectively, in order to determine the scaling factor and place the relative cross sections for the $1 \pi_{u}^{-1}$ and $80^{-1}$ ionic channels on an absolute scale. The ratio of photoelectron intensities measured at $0^{\circ}$ and $54.7^{\circ}$ determines the asymmetry paraneter. This ratio was corrected for the relative efficiency of the two detectors in a calibration procedure which has been discussed in detail. 24 
Results and discussion for the $A^{2} \pi_{u}\left(1 \pi_{u}^{-1}\right)$ channel of $\mathrm{CO}_{2}$ and the $C^{2} \Sigma^{+}\left(80^{-1}\right)$ channel of OCS are presented in Sections $B$ and $C$, respectively.

8. $\mathrm{CO}_{2} A^{2} \Pi_{u}\left(1 \pi_{u}^{-1}\right)$ Results and Discussion

The ground electronic state of $\mathrm{CO}_{2}$ can be written $\left(1 \sigma_{g}\right)^{2}\left(1 \sigma_{u}\right)^{2}\left(3 \sigma_{g}\right)^{2}\left(2 \sigma_{u}\right)^{2}\left(4 \sigma_{g}\right)^{2}\left(3 \sigma_{u}\right)^{2}\left(1 \pi_{u}\right)^{4}\left(1 \pi_{g}\right)^{4} \quad 1_{\Sigma_{g}}^{+}$. We present partial cross sections, branching ratios, and asymmetry parameters for the photoionization of $\mathrm{CO}_{2}$ into the $\mathrm{CO}_{2} A^{2} \pi_{u}\left(1 \pi_{u}^{-1}\right)$ channel.

This work addresses the first five symmetric vibrational channels of the $1 \pi_{u}^{-1}$, viz. $(n, 0,0)$, for $0 \leq n \leq 4$. From the high-resolution spectra of $\mathrm{CO}_{2}$, Potts and Fattahallah ${ }^{25}$ have assigned these levels binding energies of $17.31,17.45,17.59,17.72$, and $17.86 \mathrm{eV}$, respectively. Time-of-Flight spectra of $\mathrm{CO}_{2}$ taken at $19.3 \mathrm{eV}$ photon energy are shown in Fig. 1. Comparison of intensities measured at $0^{\circ}$ and $54.7^{\circ}$ shows asymmetries that are vibrational-state dependent.

The vibraticnally-unresolved branching ratio for the $A / B$ ionic channels of $\mathrm{CO}_{2}$ are included in Table I for a few selected photon energies. The $A / B$ biranching ratio generally increases monotonically with increasing photon energy. The brancning ratio at 21.2 ev measured by Samson and Gardner, ${ }^{28}$ including the statistical uncertainty, is within the range of the present results. In Table II the He I resonance 1 amp asymmetry parameter measurements are presented along with our results. There is excellent agreement by all groups for the vibrationsily- 
averaged asymmetry parameters. The He I resonance lamp measurenents of vibrational branching ratios and asymetry parameters are also presented in Table II. Good agreement is found among all the data for the vibrationally-resolved asymetry parameters.

Previous measurements of partial cross sections 8,11 could not completely separate the $A^{2} \pi_{U}$ and $B^{2} \Sigma_{u}^{+}$peaks, and early experimenters were obliged to sum over the $A$ and $B$ states. The present measurements have significantly higher resolution, with well separated $A$ and $B$ states. To deconvolute the vibrational peaks of the $A^{2} \pi_{U}$ channel, two methods of analysis were performed. A grid search routine ${ }^{26}$ was used when the peaks were only slightly overlapping. When the vibrational peaks were more severely overlapping, a simplex algorithm ${ }^{27}$ was used to supply the initial guesses of peak parameters for a nonlinear least square fitting routine. In both cases the peak shapes were assumed to be Gaussian.

The vibrationally-avera! i results for the $A^{2} \Pi_{u}$ channel of $\mathrm{CO}_{2}^{+}$will be presented first, followed by the vibrationaliyresolved results. In fig. 2 the vibrationally-averaged partial cross section and asymmetry parameter are illustrated. The top panel shows the fixed nuclei STMT results, ${ }^{20}$ the HF velocity and HF configuration interaction (HFCI) results, ${ }^{19}$ and the dipole $(e, 2 e)$ measurements ${ }^{11}$ compared to the present measurements. The $(e, 2, e)$ experiment could not compietely resolve the $A^{2} \Pi_{U}$ and $B^{2} \Sigma_{U}^{+}$ionic channels. Instead, the total cross sections for the $A^{2} \pi_{u}$ and $B^{2} \Sigma_{u}^{+}$were integrated and the branching ratio of the $A^{2} \Pi_{U}: B^{2} \Sigma_{u}^{+}$taken from Samson and Gardner ${ }^{2 B}$ 
was used to represent the $(e, 2 e)$ results for photon energies of 21.2 and $23.1 \mathrm{eV}$.

The cross section data show a weak rilaximum near $21 \mathrm{eV}$ and a slow decrease from 20 to $30 \mathrm{eV}$. The maximum is interpreted as a shape resonance in the $1 \pi_{u}^{-1}$ ionic channel. The MSM calculations of Swanson et al. ${ }^{1}$ suggest that the maximum in the cross section near $20 \mathrm{eV}$ is due to a $" g$ shape resonance. The STMT results of Padial et al. ${ }^{20}$ predict a resonance for the $A^{2} \Pi_{u}$ ionic channel near 20 ey photon energy, and the HF results of Lucchese and McKoy ${ }^{19}$ suggest a resonance at $22 \mathrm{eV}$. Both theories conclude that the $s$ ionic channel $\left(1 \pi_{u} \rightarrow k \delta\right)$ experiences the resonance. The STMT results of Padial et al. ${ }^{20}$ predict the position and qualitative shape of the maximum, but their value for the cross section is too high. Both HF calculations overestimate the cross section. Because the STMT calculations used a triplet static-exchange potential for the photoionization of the $1 \pi_{u}$ instead of using a singlet static exchange potential as in the $\mu$ : calculations, there is a discrepancy in the energy of the maximum in the cross section. 19

The asymmetry parameter results for the vibrationally-averaged $\mathrm{A}^{2} \Pi_{\mathrm{u}}$ channel of $\mathrm{CO}_{2}$ based on the fixed-nuclei MSM calculations of Swanson et al., 1 and Grimm et al. ${ }^{6}$, the HF calculations of Lucchese and McKoy, ${ }^{19}$, the synchrotron results of Grimm et al., 6 and the present results are displayed in the bottom panel of Fig. 2. There is good agreement with the theoretical results ${ }^{6,19}$ and with the data of Grimm et al. ${ }^{6}$ The experimental results, as well as the 
theoretical predictions, show an increase in the asymmetry parameter from threshold to an asymptotic value of 1.

Results for the ground vibration channel $(0,0,0)$ of the $A^{2} i_{u}$ ionic channel are shown in Fig 3. In the top panel the partial cross section is presented. A maximum near $21 \mathrm{eV}$ is probably evidence of the effect for the shape resonance on the cross section, predicted by Swanson et at. 1

The asymetry parameter for the $(0,0,0)$ vibrational channel measured by Grim et al. ${ }^{6}$ is displayed with the present results in the bottom panel of Fig. 3. There is excellent agreement with the measurements of Grimm et al. ${ }^{6}$ Both show the weak maximum itructure near $21 \mathrm{eV}$ photon energy in the asymnetry parameter, which is proposed to be due to a shape resonance. The theoretical results of Swanson et al., 1 shown by the solid curve, are also in good over all agreenent with experiment.

The remainder of the vibrationally-resolved data for the $A^{2} \Pi_{U}$ ionic state of $\mathrm{CO}_{2}$ are shown in figs. 4-7. The MSM resuits of Swanson et al. 1 for vibrationally-resolved branching ratios, which account for both Franck. ndon and non-Franck-Condon effects, are i) satrated alongside the present measurements in the top panels of these figures. The vibrationally-resolved asymmetry parameter results of Grimm et a $1 .{ }^{6}$ and the predictions based on a MSli calculation 1 are included in the bottom panels of these figures.

The vibrationally-resolved results for the $(1,0,0)$ vibrational channel of the $A^{2} \Pi_{U}$ channel of $\mathrm{CO}_{2}$ are displayed in Fig 4. The 
non-Franck Condon MSM results predict two very shallow maxima in the branching ratio. The Franck-Condon branching ratio is independent of photon energy. The experimental branching ratio shows two large maxima located near 21 and $24 \mathrm{eV}$ photon energy, and the branching ratio is between 2 and 3 . We attribute the first feature to the shape resonance effect in the $A^{2} \Pi_{U}$ ionic channel. The other feature could result from the $\sigma_{u}$ shape resonance as predicted by Swanson et al. ${ }^{1}$ or from a resonance in the $1 \pi_{u} \rightarrow k_{g}$ subchannel as suggested by Padial et al. ${ }^{20}$ and Lucchese and McKoy. 19

The theoretical results predict vibrational branching ratios higher than the measurements. In addition, the observed modulatation of the branching ratio with photon energy is absent in the FranckCondon approximation and is very small in the non-Franck-Condon calculation.

The asymmetry parameter for the $(1,0,0)$ channel is presented in the bottom panel of Fig. 4. Again there is excellent agreement with the measurements of Grim et al. ${ }^{6}$ and good agreement with the MSM results of Swanson et al. 1 The experimental results show variations in the 21-22 el photon energy range, possibly attributable to a shape resonance.

The results for the $(2,0,0)$ vibrational channel are displayed in Fig. 5. The experimental vibrational branching ratio takes values between 2 and 3.5. This channel is the most intense peak over most of the photon energy range of $18-25 \mathrm{eV}$. The theoretical results are in only fair agreement with the experimental measurements. 
The asymmetry paraneter for the $(2,0,0)$ channel is displayed in the buttum painal of Fig. 5. The sgreement with the MSM predictions and asymetry paraneter measured by $\operatorname{Grimm}^{6}$ for the $(2,0,0)$ channel are similar to that for the $(1,0,0)$ channel. A possible broas shape resonance falls near 22 el photon energy. The MSM calculation has significantly higher values near threshold than the data.

Results for the $(3,0,0)$ channei are presented in Fig. 6 . The experimental vibrational ratio takes values between 2 and 3 . The vibrational branching ratio shows a probable maximum in the 20-21 $\mathrm{EV}$ region. Scatter in the data preclude further conclusions.

In the bottom panel of Fig. 6 the asymmetry parameter of the $(3,0,0)$ is displayed. Again there is excellent agreement with the measurements of Grimm et al. ${ }^{6}$ and good agreement with the MSM calculations of Swanson et al. ${ }^{1}$. Like the previous vibrational channels the MSM results predict higher asymmetry values than are experimentally measured. A weak feature near $20-21 \mathrm{eV}$ is present in our data and the data of Grim et al. 6

The last vibrational channel resolved in the present study of the $A^{2} \Pi_{u}$ ionic channel is the $(4,0,0)$ channel, for which results are displayed in Fig. 7. In the top panel the measured vibrational ratio takes values between 1 and 2.3. Similar to all the other vibrational channels, the non-Franck-Condon predictions based on the MSM calculations of Swanson et al. ${ }^{1}$ suggest that two maxima are present for the vibrational branching ratio of the $(4,0,0)$ channel. Two maxima appear to be present in our data, near 20 and $24 \mathrm{el}$. The maxima are probably due to shape resonances. 
In the bottom panel of fig. 7 the agreement between our results and that of Grim et al. 6 and the theoretical MSM results of Swanson et al. ${ }^{1}$ is similar to the comparisons made for the other vibrational states. The MSM asymmetry parameters, similar to the other channels are higher than the measurements near threshold.

In summary, we can draw a few conclusions about the vibrationally-resolved results for the $A^{2} \pi_{u}$ ionic channel of $\mathrm{CO}_{2}$.

(1.) The synchrotron radiation results of Grim et al. ${ }^{7}$ are in very good agreement with the present data.

(2.) The vibrationally-resolved branching ratios show non-Franck Condon behavior, as suggested by Swanson et al. ${ }^{1}$ Their MSM calculations predict two maxima in the vibrational branching ratios of all the vibrational channels. This is qualitatively confirmed by our results, but quantitative agreement is missing.

(3.) The non-Franck-Condon results of Swanson et a $1 .^{1}$ for the vibrationally-resolved asymetry parameters for the $A \mathbb{1}_{u}$ of $\mathrm{CO}_{2}$ are generally in good agreement with the present measurements. However, near threshold the theoretical results predict asymetries higher than the measured asymmetries. 
C. OCS $\mathrm{C}^{2} \Sigma^{+}\left(8 \sigma^{-1}\right)$ Results and Discussion

The ground electronic state of OCS can be written $(1 \sigma)^{2}(2 \sigma)^{\overline{2}}(3 \sigma)^{\hat{2}}(4 \sigma)^{\hat{2}}(52 p)^{\hat{\sigma}}(6 \sigma)^{\hat{2}}(7 \sigma)^{\hat{2}}(8 \sigma)^{2}(9 \sigma)^{2}(2 \pi)^{4}(3 \pi)^{4} \frac{1}{\Sigma}^{+}$. The valence-shell spectrum resulting from the ionization of the last four orbitals has recently been studied theoretically $7,12,29$ and experimentally. $7,10,23,31$ We present the first vibrationallyresolved studies of the $\mathrm{C}^{2} \Sigma \Sigma^{+}$channel over the photon energy range $h v=19-24 \mathrm{ev}$. The vibrationally-averaged OCS measurements were performed separately, over the photon energy range of 20.5-29 eV. The ocs $c^{2} \Sigma^{+}$photoelectron peaks were fitted in the same way as the $\mathrm{CO}_{2} \mathrm{~A}^{2} \Pi_{\mathrm{u}}$ peaks. The collection time per spectrum was typically 2000 seconds.

Time-of-flight photoelectron spectra of the $c^{2} \Sigma^{+}\left(8 \sigma^{-1}\right)$ ionic channel of OCS, converted to an linear energy scale, are displayed in Fig. 8. From left to right the peaks correspond to the $(0,0,2),(1,0,1)$, $(0,0,1),(1,0,0)$, and $(0,0,0)$ vibrational channels with binding energies of $18.5,18.34,18.23,18.08$, and $17.96 \mathrm{eV}$, respectively. ${ }^{10}$ The quality of these spectra are comparable to those obtained by Delwiche et a1. 10 It is very apparent that the asymmetry parameter of the $(1,0,0)$ channel is significantly oifferent from all the other channels, as noted by Carlson and McGuire, ${ }^{16}$

Our vibrationally-averaged and vibrationally-resolved asymetry parameters for the $C^{2} \Sigma^{+}$ionic channel, along with the He I resonance lamp measurements of Carlson and McGuire, ${ }^{16}$ are presented in Table III. The two experiments show good agreenent. 
The vibrationally-averaged partial cross section results for the $c^{2} \Sigma^{+}$ionic channel of OCS from MSH calculations performed by Grimm et al. ${ }^{12}$ synchrotron radiation measurements of Carlson et al., 7 dipole $(e, 2 e)$ results of thite et al.,$^{23}$ and the present results are presented in the top panel of Fig. 9. No obvious structure is apparent in the synchrotron radiation cross section measurements, in contrast to the $(e, 2 e)$ results. Th partial cross section is fairly constant over the photon energy range of 20-29 eV. There is good agreement with the cross section results of Carlson et al. ${ }^{7}$ However, there is poor agreement with the $(e, 2 e)$ results of White et al. ${ }^{23}$ and the cross section predicted based on MSM calculations by Carison et al. 7

The vibrationally-averaged asymmetry parameter for the $C^{2} \Sigma^{+}$ ionic channel measured by Carison et al., 7 and by us is presented in the bottom panel of Fig. 9. The measured as ymmetry parameter increases slowly with increasing photon energy. There is good agreement with the results of Carlson et al., ${ }^{7}$ but poor agreement with the asymmetry parameter predicted by Grimm et al. 12

The vibrationally-resolved results for the $v_{0},(0,0,0)$ ionic channel are displayed in Fig 10. In the top panel, the partial cross section increases slowly with photon energy. The asymmetry data and the He I resonance line result $t^{16}$ are illustrated in the bottom panel of Fig. 10.

The oCs $C^{2} \Sigma^{+}$ionic channel vibrational ratios are displayed in the top pane1s, and the He I resonance line results along with the present results are presented in the bottom panels, of Figs. 11-13. 
The vibrationally-resolved results for the $(1,0,0) \mathrm{c}^{2} \Sigma^{+}$ionic channel are presented in Fig. 11. In the top panel, the vibrational branching ratio generaily decreases with imcreasfing photon energy, with a possible maximum at the low energy end.

The asymmetry parameter for the $(1,0,0)$ channe? is presented in the bottom panel of Fig. 11. The asymmetry parameter increases from -0.5 to 0.5 over the photon energy range 19 to $24 \mathrm{eV}$. There is some discrepancy present between the He I results ${ }^{16}$ and the present results. Nonetheless, we confirm that the asymmetry Darameter for the $(1,0,0)$ is dissimilar to the $(0,0,0)$ vibrational channel, as discussed by Carlson and McGuire. ${ }^{16}$ The reason for this different behavior remains unexplained.

Vibrationally-resolved resuits for the $(0,0,1)$ asymmetric stretch vibrational channel of the $c^{2} \Sigma^{+}$ionic channel of ocs are displayed in Fig. 12. The vibrational branching ratio for this channel is presented in the top panel. Its behavior is similar to that of the $(1,0,0)$ channe?.

The asymmetry parameter for the $(0,0,1)$ channel is displayed in the bottom panel of fig. 12. The asymmetry parameter increases slowly from 19-24 ev. It is difficult to identify any resonance structure because of the scatter in the data. There is excellent agreement with the He I resonance line measurement ${ }^{16}$ for this channel.

The final vibrationally-resolved channel that we studied was the $(0,0,2)$ channel. Measurements for this state from 19-22 eV are presented in the top panel of Fig. 13. The branching ratio is very small $(-0.05-0.10)$, and the scatter of the data preclude identifying any trends. 
The asymmetry parameter for the $(0,0,2)$ channel is displayed in the bottom panel of Fig. 13. A flat dependence for the asymmetry parameter is observed. There is excellent agreement with the He I resonance line result. ${ }^{16}$ The energy dependence of this asyminetry parameter is yory similar to the $(0,0,0)$ and $(0,0,1)$ vibrationat channels, in contrast to the $(1,0,0)$ channel.

The conclusions we can draw about the vibrational-resolyed results for the $C^{2} \Sigma^{+}$ionic channel of OCS are as follows:

(1.) There is good to excellent agreement with the vibrationallyaveraged and vibrationally-resolved He 1 resonance line results of Carlson and McGuire. ${ }^{16}$

(2.) The synchrotron radiation results of Carlson et al. ${ }^{7}$ are in good agreement with present measurements for the vibrationallyaveraged partial cross section and asymetry parameter for the $c^{2} \Sigma^{+}$ionic channel of ocs.

(3.) The vibrationally averaged partial cross sections obtained from $(e, 2 e)$ measurements of white et al. ${ }^{23}$ are in poor agreement, probably because their measurements have lower energy resolution and lower counting statistics than the present measurements. 
(4.) The vibrationally-ayeraged cross section and asymetry parameter for the $c^{2} \Sigma^{+}$ionic channel of OCS do not appear to have resonance features.

(5.) The vibrationally-averaged results based on MSM calculations of Grim et a1. ${ }^{12}$ and Carlson et al. ${ }^{7}$ are in poor agreenent with the measurements.

(6.) There seem to be maxima in the vibrational branching ratios for the $(1,0,0)$ and $(0,0,1)$ vibrational channels of the $c^{2} \Sigma^{+}$ionic channel of ocs.

(7.) The angular nature of the $(1,0,0)$ vibrational channel in forming the oCS $C^{2} \Sigma^{+}$state is significantiy different from that of all the other vibrational channels. 


\section{References}

1. J.R. Swanson, D. Dill, and J.L. Dehmer, J. Phys. B: At. Mol. Phys. 14. L207 (1981).

2. Ch. Jungen and O. Altebek, J. Chem. Phys. 66, 5584 (1977); Ch. Jungen and D. Dill, J. Chem. Phys. 73, 3338 (1980).

3. Y. Itikawa, Chem. Phys. Lett. 62, 261 (1979); Y. Itikawa, Chem. Phys. 37, 410 (1979).

4. A.C. Parr, D.L. Ederer, B.E. Cole, J.B. West, R. Stockbauer, K. Codling, J.L. Dehmer, Phys. Rev. Lett. 46, 22 (1981); A.C. Parr, D.L. Ederer, J.L. Dehmer, and D.M.P. Holland, J. Chem. Phys. 77, 111 (1982); P. Morin, I. Nenner, M.Y. Adam, M.J. Hubin-Franskin, J. Delwiche, H. Lefebrre-Brion, and A. Giusti-Suzor, Chem. Phys. Lett. 92, 609 (1982); P. Mor in, I. Nenner, P.M. Guyon, L.F.A. Ferreira, and K. Ito, Chem. Phys. Lett. 92, 103 (1982); C.M. Truesdale, S. Southworth, P.H. Kobrin, D.W. Lindle, and D.A. Shirley, to be published in J. Chem. Phys. (1983).

5. C.N. Yang, Phys. Rev. 74, 764 (1948); J. Cooper and R.N. Zare, J. Chem. Phys. 48, 942 (1968).

6. F.A Grim, J.D. Allen, Jr., T.A. Carlson, M.O. Krause, D. Mehaffy, P.R. Keller, and J.W. Taylor, ibid. 75, 92 (1981).

7. T.A. Carlson, M.0. Krause, and F.A. Grimm, J. Chem. Phys. 77, 1701 (1982).

8. T. Gustafsson, E.W. Plummer, D.E. Eastman, and W. Gudat, Phys. Rev. A 17,175 (1978).

9. T.A. Carlson, M.0. Krause, and F.A. Grimm, J. Chem. Phys. 77, 
$701(1982)$

10. J. Delwiche, M.J. Hubin-Franskin, P.M. Guyon, and I. Nenner, J. Chem. Phys. 74, 4219 (1981).

11. C.E. Brion and K.H. Tan, J. Electron Spectrosc. Relat. Phenom. 15, $241(1979)$.

12. F.A. Grimm, T.A. Carlson, W.B. Dress, P. Agron, J.0. Thomson, and J.W. Davenport, J. Chem. Phys. 72, 3041 (1980).

13. M.H. Kibel, F.J. Leng, and E.L. Nyberg, J. Electron Spectrosc. Relat. Phenom. 15, 281 (1979).

14. W.H. Hancock, J.A.R. Samson, ibid. 9, 211 (1976).

15. T.A. Carlson, G.E. McGuire, A.E. Jonas, K.L. Cheng, C.P. Anderson, C.C. Lu, and B.P. Pullen, in Electron Spectroscopy, ed. D.A. Shirley (North Holland, Amsterdam (1972)) p. 207.

16. T.A. Carlson and G.E. McGuire, J. Electron Spectrosc. Relat. Phenom. 1, 209 (1972/73).

17. S. Katsumata, Y. Achiba, and K. Kimura, ibid. 17, 229 (1979).

18. J. Kriele and A. Schweig, ibid. 20, 191 (1980).

19. R.R. Lucchese and V. McKoy, Phys. Rev. A 26, 1406 (1982).

20. N. Padia], G. Csariak, B.V. McKoy, P.H. Langhoff, ibid. 23, 218 (1981).

21. M.G. White, R.A. Rosenberg, G. Gabor, E.D. Poliakoff, G. Thornton, S.H. Southworth, and D.A. Shirley, Rev. Sci. Instrum. 50, $1288(1979)$.

22. A.P. Hitchcock, C.E. Brion, and M.J. van der Wiel, Chem. Phys. 45, $461(1980)$. 
23. M.G. White, K.T. Leung, and C.E. Brion, J. Electron Spectrosc. Relat. Phenom. $\underline{23}, 127$ (1981).

24. S. Southworth, C.M. Truesdale, P.H. Kobrin, D.H. Lindle, H.D. Brewer, and D.A. Shirley, J. Chem. Phys. 76, 143 (1982).

25. A.W. Potts and G.H. Fattahallah, J. Phys. B: Atom. Molec. Phys. $\underline{13}$, 2545 (1980).

26. P.R. Bevington, Data Reduction and Error Analysis for the Physical Sciences (McGraw Hill, New York, 1969),D. 208.

27. J.A. Nelder and R. Mead, Computer J. Z, 308 (1965); R. O'Neill, Applied Statictics 20, 338 (1971).

28. J.A.R. Samson and J.L. Gardner, J. Geophys. Res, 78, 3663 (1973).

29. J.P.D Cook, M.G. White, C.E Brion, W. Doncke, J. Schirmer, L.S Cederbaum, and W. Von Niesson, J. Electron Spectrosc. Relat. Phenom. 22, 261 (1981). 
Table I. Branching ratios of the $A^{2} \Pi_{u}\left(1 x_{u}^{-1}\right)$ : $B^{2} \Sigma_{u}^{+}$ionic channels of $\mathrm{CO}_{2}$.

\begin{tabular}{llll}
\hline hu(eV) & $A / B^{\mathrm{a}}$ & hv(eV) & A/B \\
\hline 18.7 & $1.13(12)$ & 22.3 & $0.60(7)$ \\
19.3 & $0.75(3)$ & 22.6 & $0.81(8)$ \\
20.0 & $0.70(7)$ & 22.8 & $1.28(15)$ \\
20.3 & $0.70(8)$ & 23.1 & $0.74(7)^{\mathrm{b}}$ \\
20.6 & $0.74(9)$ & 23.8 & $0.89(6)$ \\
21.2 & $0.80(10)$ & 24.3 & $1.14(10)$ \\
& $0.65(5)^{\mathrm{b}}$ & 24.8 & $1.05(11)$ \\
21.6 & $0.75(8)$ & 26.0 & $1.15(10)$ \\
& & & \\
\hline a. Errors in the last digit given parenthetically. & \\
b. Ref. 28. & & \\
\hline
\end{tabular}


Table II. Comparison of He I $(21.2 \mathrm{eV})$ resonance lamp vibrationally-averaged and resolved asymmetry parameter for the $\AA^{2} \Pi_{u}$ channel of $\mathrm{CO}_{2}$ with the present measurements.

\begin{tabular}{|c|c|c|c|c|c|c|}
\hline & avg. $\beta$ & $B\left(y_{0}\right)^{d}$ & $B\left(v_{1}\right)$ & $B\left(v_{2}\right)$ & $s\left(v_{3}\right)$ & $v\left(v_{4}\right)$ \\
\hline Ref. 12 & - & - & -..- & $1.01(5)$ & $-\infty$ & - \\
\hline Ref. 13 & 0.80 & --.- & ---- & - & - & -... \\
\hline Ref. 14 & ---- & $0.80(5)$ & $0.80(5)$ & $0.80(5)$ & $-\infty$ & $--m$ \\
\hline Ref. 15 & - & $0.70(10)$ & $0.90(7)$ & - & $\ldots$ & -- \\
\hline Ref. 16 & $0.80(10)$ & $0.90(20)$ & - & $0.80(10)$ & $0.70(5)$ & $0.60(10)$ \\
\hline Ref. 17 & ---- & --- & - & $0.83(8)$ & - & $=$ \\
\hline Ref. 18 & $0.72(3)$ & $0.72(3)$ & $0.88(5)$ & $0.78(3)$ & $0.63(2)$ & $0.49(2)$ \\
\hline Present & $0.71(6)$ & $0.85(5)$ & $0.85(5)$ & $0.80(6)$ & $0.55(10)$ & $0.50(7)$ \\
\hline
\end{tabular}

d. The $v_{0}-v_{4}$ designations refer to the symmetric modes $(0,0,0),(1,0,0),(2,0,0)$, $(3,0,0)$, and $(4,0,0)$ of the $A^{2} \pi_{u}\left(1 \pi^{-1}\right)$ ionic channel of $\mathrm{CO}_{2}$. 
Table III. Comparison of the I $(21.2 \mathrm{eV})$ resonance source vibrationally-averaged and resoived asymmetry parameter for the $C^{2} \Sigma^{+}$channel of OCS with the present measurements.

$$
\text { avg.s } \quad s\left(v_{0}\right)^{\mathrm{a}} \quad B\left(v_{1}\right) \quad B\left(v_{2}\right) \quad s\left(v_{3}\right)
$$

$\begin{array}{lllcll}\text { Ref. 16 } & 0.55(10) & 0.55 & 0.25(7) & 0.72(8) & 0.68(10) \\ \text { Present } & 0.54(7) & 0.61(6) & -0.18(14) & 0.74(7) & 0.69(27)\end{array}$

a. The $v_{0}-v_{3}$ refer to the $(0,0,0),(1,0,0),(0,0,1)$, and $(0,0,2)$ vibrational channels of the $C^{2} \Sigma^{+}(8 \sigma-1)$ ionic channel of oCS. 


\section{4}

\section{Figure Captions}

Figure 1. Time-of-flight spectra of $\mathrm{CO}_{2}$ taken at a photon energy of 19.3 $\mathrm{eV}$, showing the vibrationally resolved peaks of the $A^{2} \Pi_{U}$ and $B^{2} \Sigma_{u}^{+}$ionic channels. The vibrational peaks for the $A^{2} \pi_{u}$ from left to right are: $(4,0,0)$, $(3,0,0),(2,0,0),(1,0,0)$, and $(0,0,0)$. The quantum numbers refer to symmetric stretch, bending, and asymnetric stretch modes. The vibrational peaks for the $B^{Z^{2} \Sigma_{u}^{+}}$from left to right are $(1,0,0)$ and $(0,0,0)$.

Figure 2. Vibrationally-averaged $\sigma(\varepsilon)$ and $B(c)$ for the $A^{2} \pi_{u}$ ionic channel of $\mathrm{CO}_{2}$. For the o(c) curves, $(-)$ represents the STMT calculation of Padial et al., $20(-)$ the $H F$ velocity calculation of Lucchese and Mckoy, ${ }^{19}$ the solid curve is the HFCI calculation of Lucchese and McKoy, ${ }^{19}$ open circles are the dipole $(e, 2 e)$ measurements of Brion and $\operatorname{Tan}^{10}$, and filled circles are the present measurements. For the $8(\varepsilon)$ curves, the solid curve shows the fixed-nuclei MSM calculation of Swanson et al., 1 the dotted curve the fixed-nuclei HF calculation of Lucchese and McKoy, ${ }^{19}$ and the dashed curve the MSM calculation of Grim et al. ${ }^{6}$ Open circles show the measurements of Grim et al., 6 and filled circles the present measurements with statistical uncertainties.

Figure 3. Photoionization cross section and asymmetry parameter $B(\varepsilon)$ for the $(0,0,0)$ vibrational channel of the $A^{2} \pi_{u}$ ionic state of $\mathrm{l}_{2}$. Filled circles show the measured photoicilization cross 
sections. For $:(c)$, the solid curve shows the MSM calculation of Swanson et al., 1 open circles the measurements of Grim et al., 6 and filled circles the present measurements.

Figure 4. The vibrational branching ratio and $B(\varepsilon)$ for the $(1,0,0)$ vibrational channel of the $A^{2} \Pi_{u}$ ionic state of $\mathrm{CO}_{2}$. For the vibrational branching ratios, the solid curve shows the MSM calculation of Swanson et al., ${ }^{19}$ the dashed curve shows the Franck-Condon prediction, ${ }^{19}$ and the experimental results are shown by filled circles. For $B(c)$, the solid curve shows the MSM calculation of Swanson et al., 1 open circles represent the measurements of of Grimm et al., 6 and the present measurements are shown by filled circles.

Figure 5. Vibrational branching ratio and $B(c)$ for the $(2,0,0)$ vibrational channel of the $A^{2} \Pi_{u}$ ionic state of $\mathrm{CO}_{2}$. The experimental and theoretical results are denoted as in Fig. 4.

Figure 6. Vibrational branching ratio and $B(c)$ for the $(3,0,0)$ vibrational channel of the $A^{2} \pi_{u}$ ionic state of $\mathrm{CO}_{2}$. The experimental and theoretical results are denoted as in Fig. 4.

Figure 7. Vibrational branching ratio and $B(c)$ for the $(4,0,0)$ vibrational channel of the $A^{2} \Pi_{u}$ ionic state of $\mathrm{CO}_{2}$. The experimental and theoretical results are denoted as in Fig. 4.

Figure 8. Time-of-flight spectra taken at the He I energy $(21.22 \mathrm{eV})$, with a $2 \checkmark$ retarding potential, for the $c^{2} \Sigma^{+}\left(8 o^{-1}\right)$ ionic channel of OCS, after conversion to a linear energy scale. The 
photoelectron peaks from left to right are the $(0,0,2),(1,0,1)$, $(\dot{v}, 0,1),(1,0,0)$, and $(0,0,0)$ vibrational channels, with binding energies 18.5, 18.34, 18.23, 18.08, and $17.96 \mathrm{eV}$, respectively.

Figure 9. Vibrationally-averaged $\sigma(\varepsilon)$ and $s(c)$ for the $c^{2} \Sigma^{+}$ionic channel of ocS. For the $\sigma(\varepsilon)$ curves, $O$ are the dipole $(e, 2 e)$ measurements of white et al.,,$^{23}$ are the measurements of Carlson et al., ${ }^{7}$ and are the present measurements. For the $B(c)$ results in the bottom panel, $\phi$ are the measurements of Carlson et al., ${ }^{7}$ and are the present measurements.

Figure 10. $\sigma(c)$ and $B(\varepsilon)$ for the $(0,0,0)$ vibrational channel for the $c^{2} \Sigma^{+}$ionic channel of ocs. In both panels filled circles are the present measurements. In the bottom panel the open circle is the He I resonance lamp measurement of Carison and McGuire. 16

Figure 11. Vibrational branching ratio and $\beta(\varepsilon)$ for the $(1,0,0)$ vibrational channel for the $c^{2} \Sigma^{+}$ionic channel of ocs. The experimental results are denoted as in Fig. 10.

Figure 12. Vibrational branching ratio and $B(c)$ for the $(0,0,1)$ vibrational channel for the $C^{2} \Sigma^{+}$ionic channel of OCS. The experimental resuits are denoted as in Fig. 10.

Figure 13. Vibrational branching ratio and $B(c)$ for the $(0,0,2)$ vibrational channel for the $c^{2} \Sigma^{+}$ionic channel of ocs. The experimental results are denoted as in Fig. 10. 


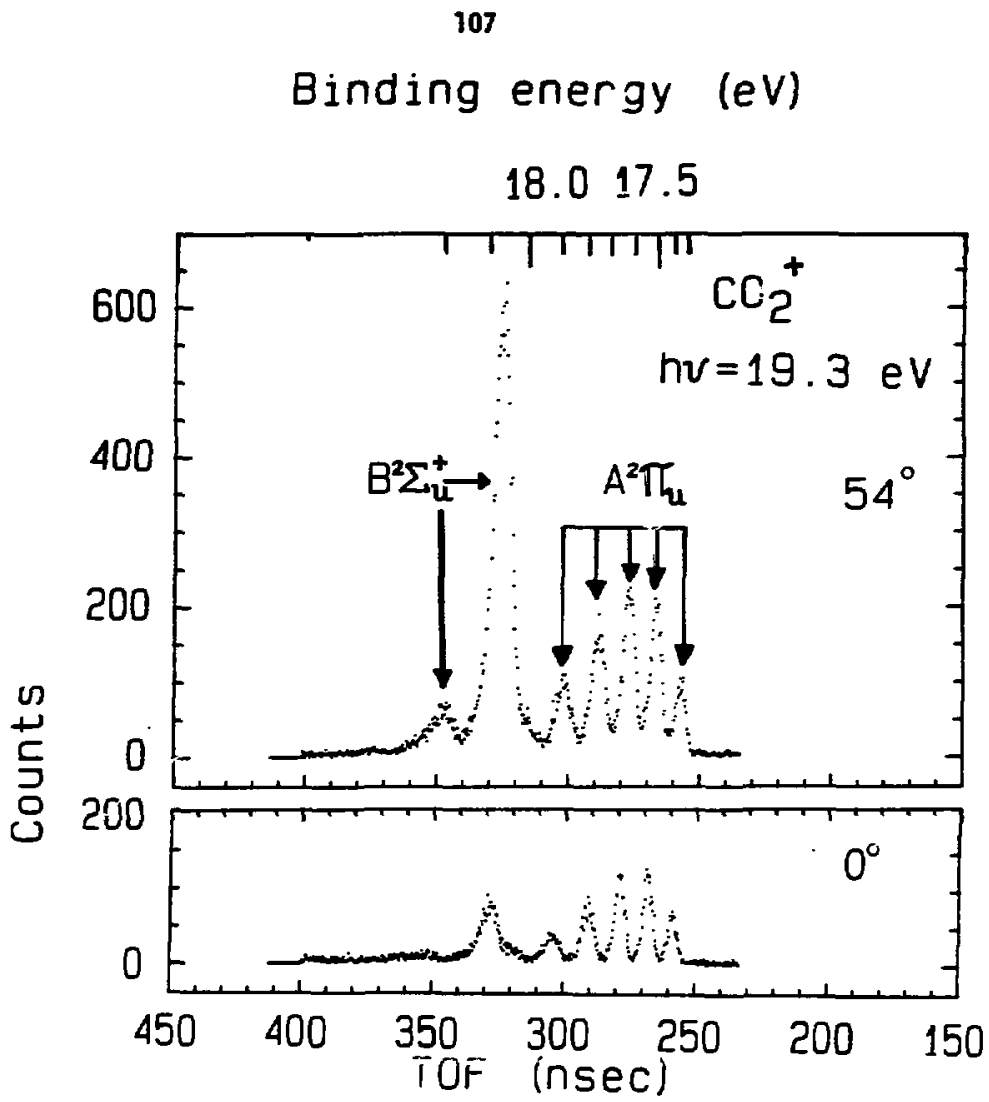

Fig. 1

XBL 833-8542 
108

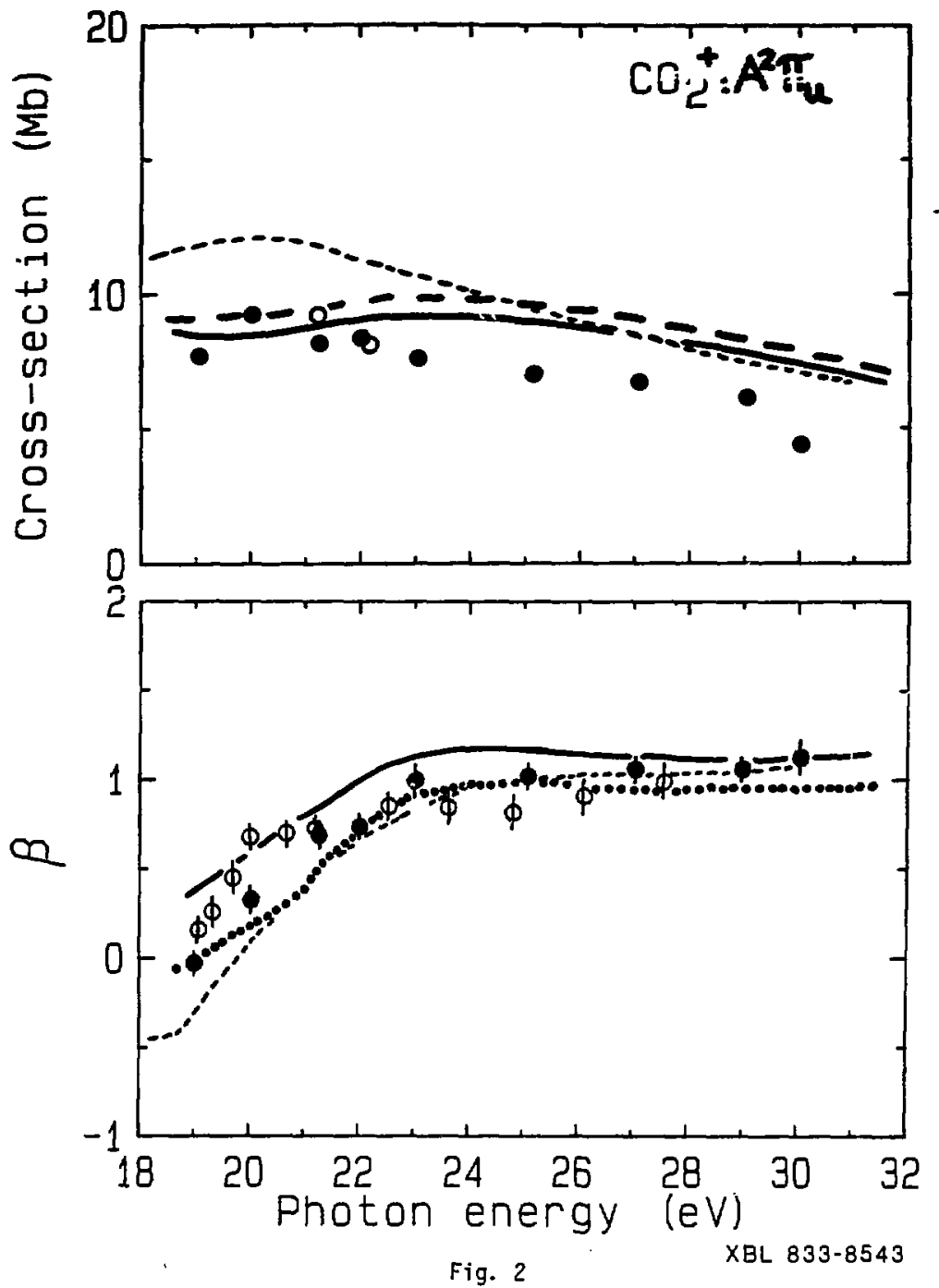


109
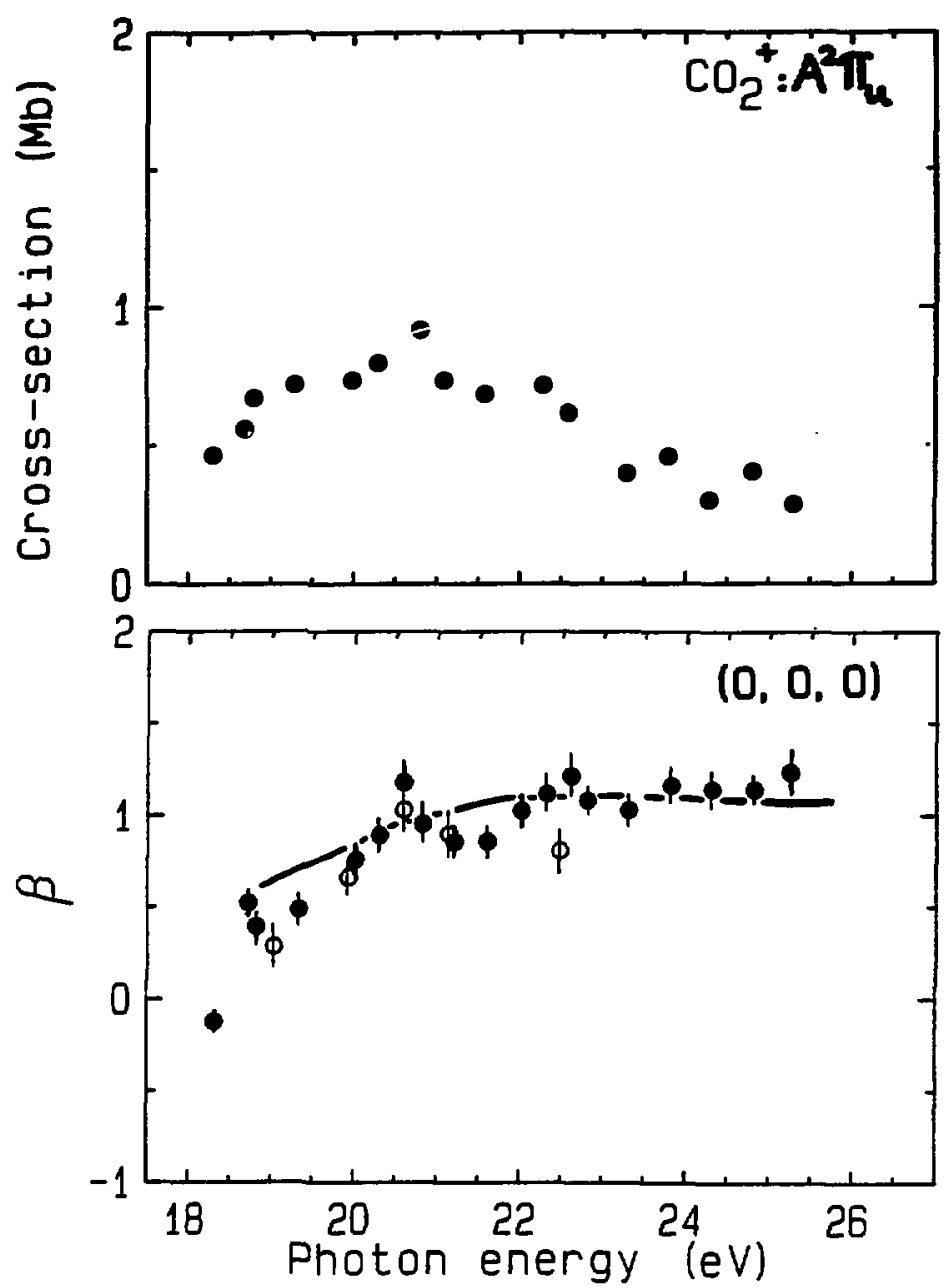

Fig. 3

XBL 833-8545 
110
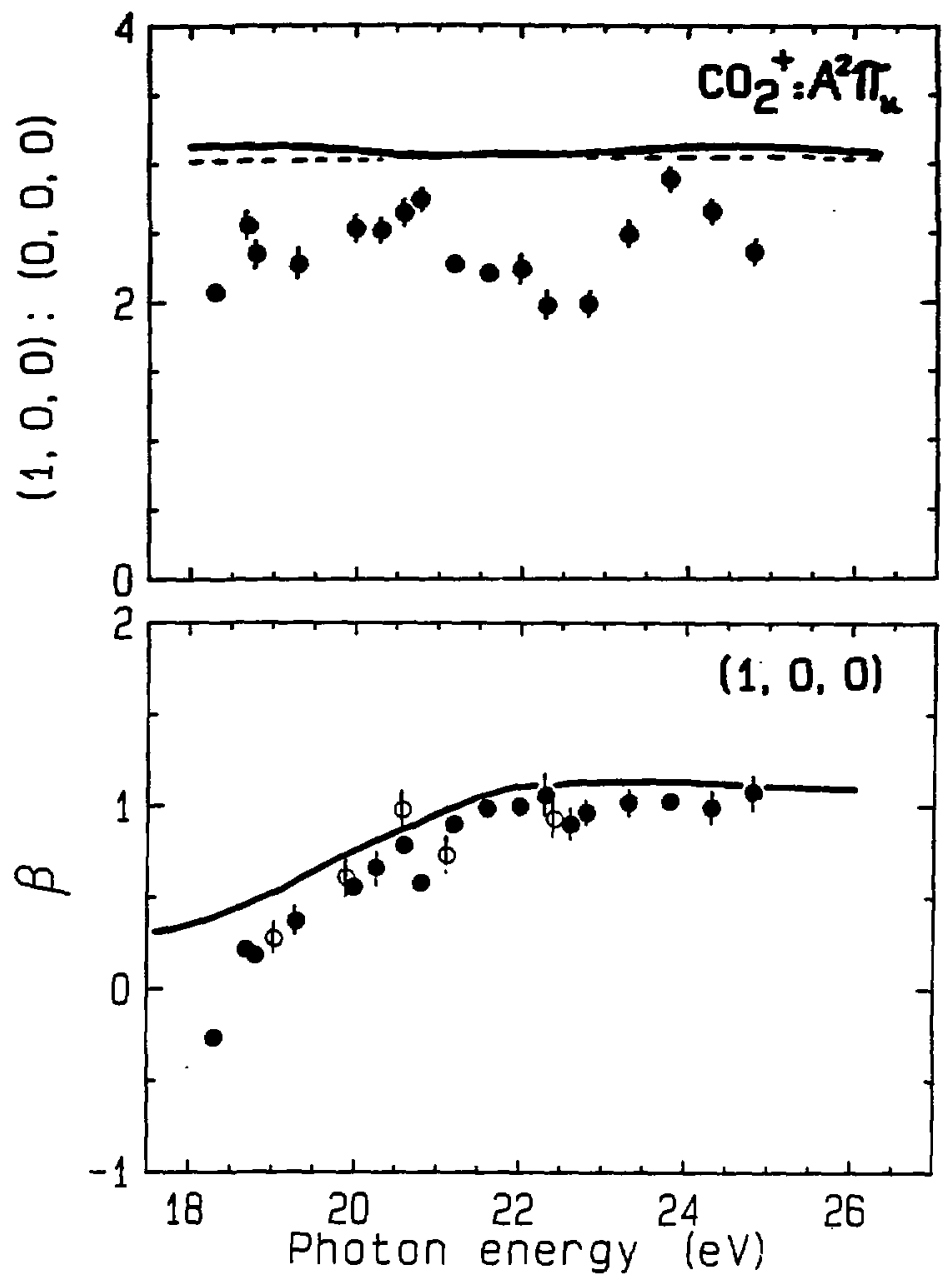

Fig. 4

XBL 833-8544 

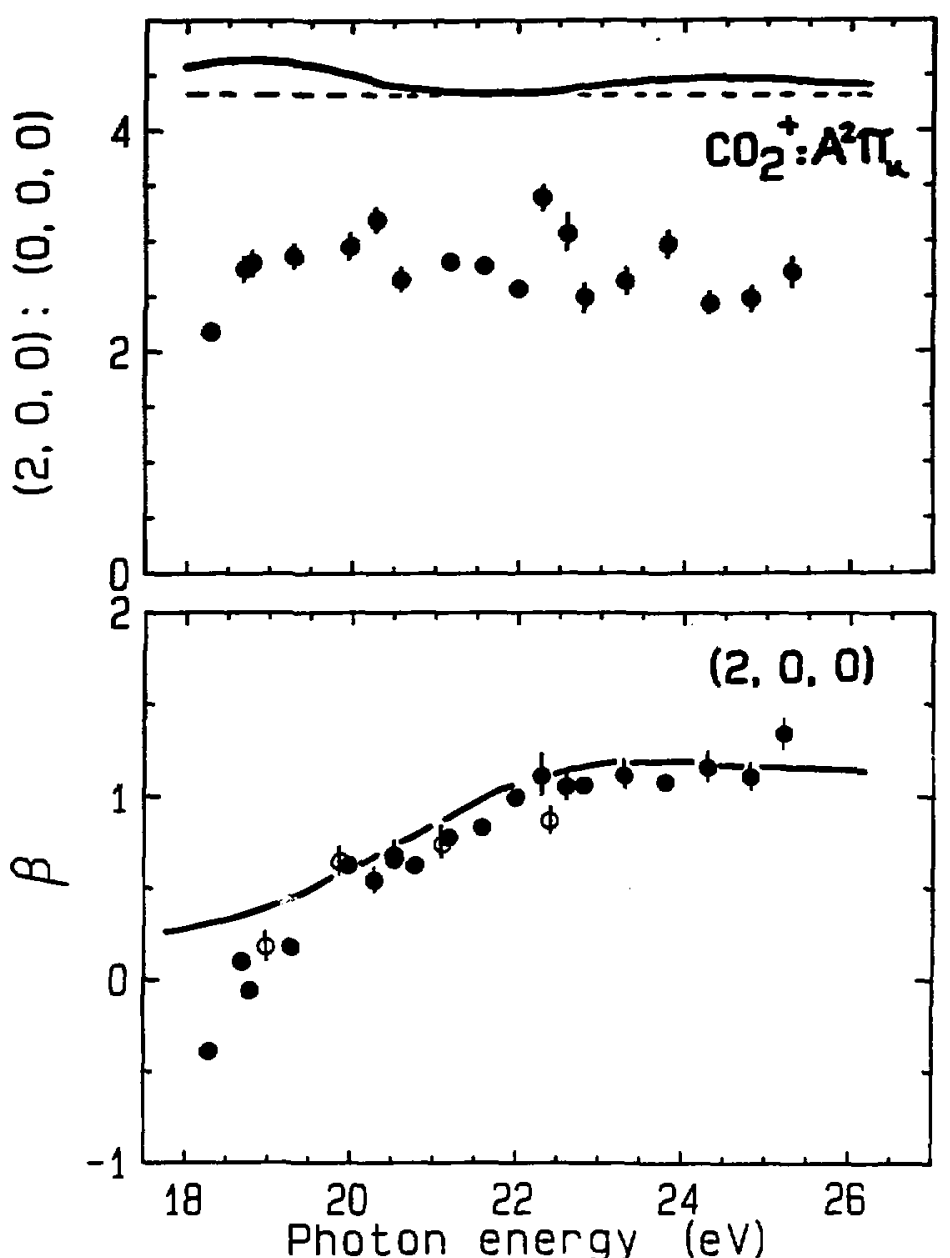

Fig. 5

XBL 833-3546 


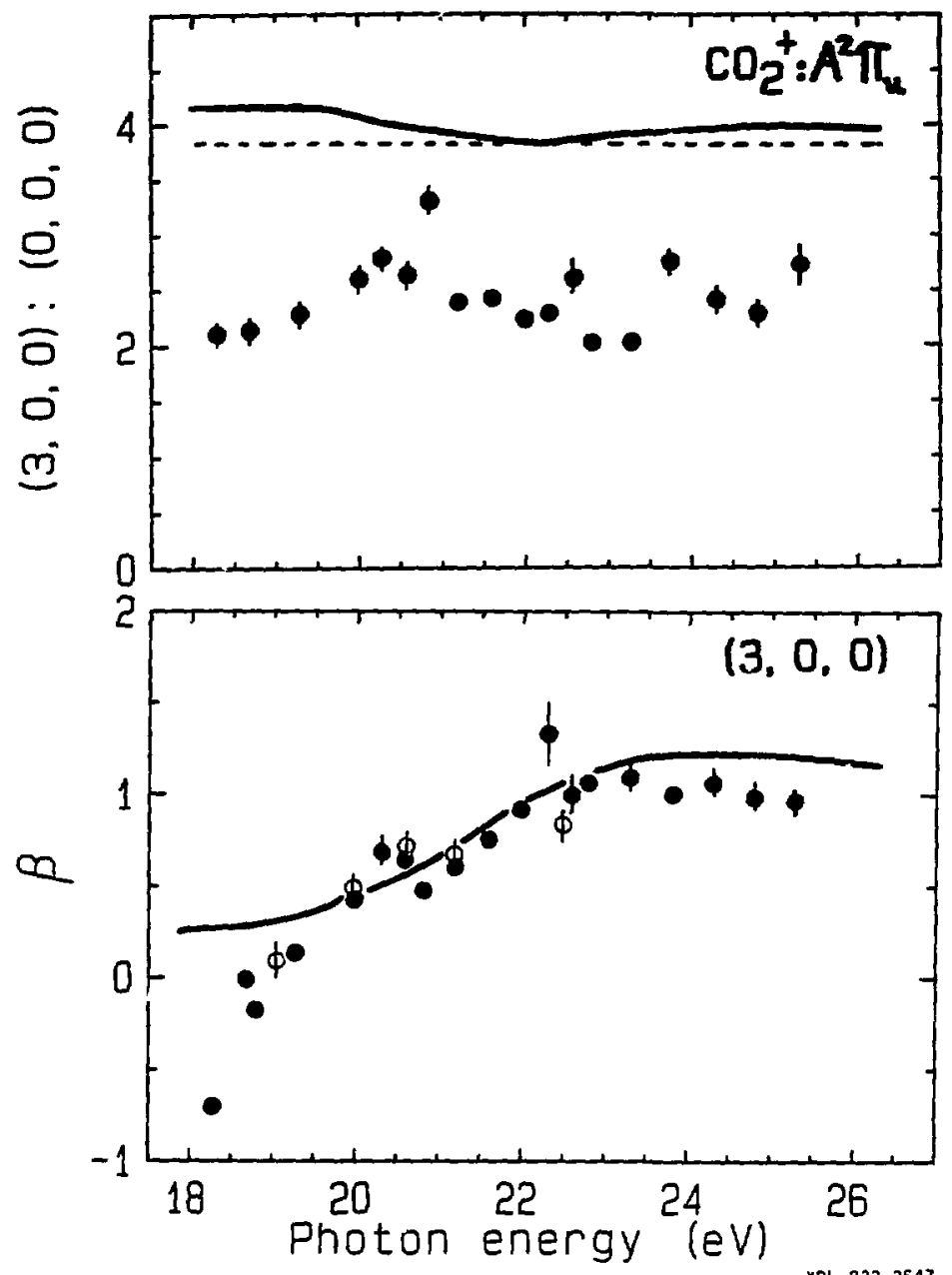

Fig. 6 


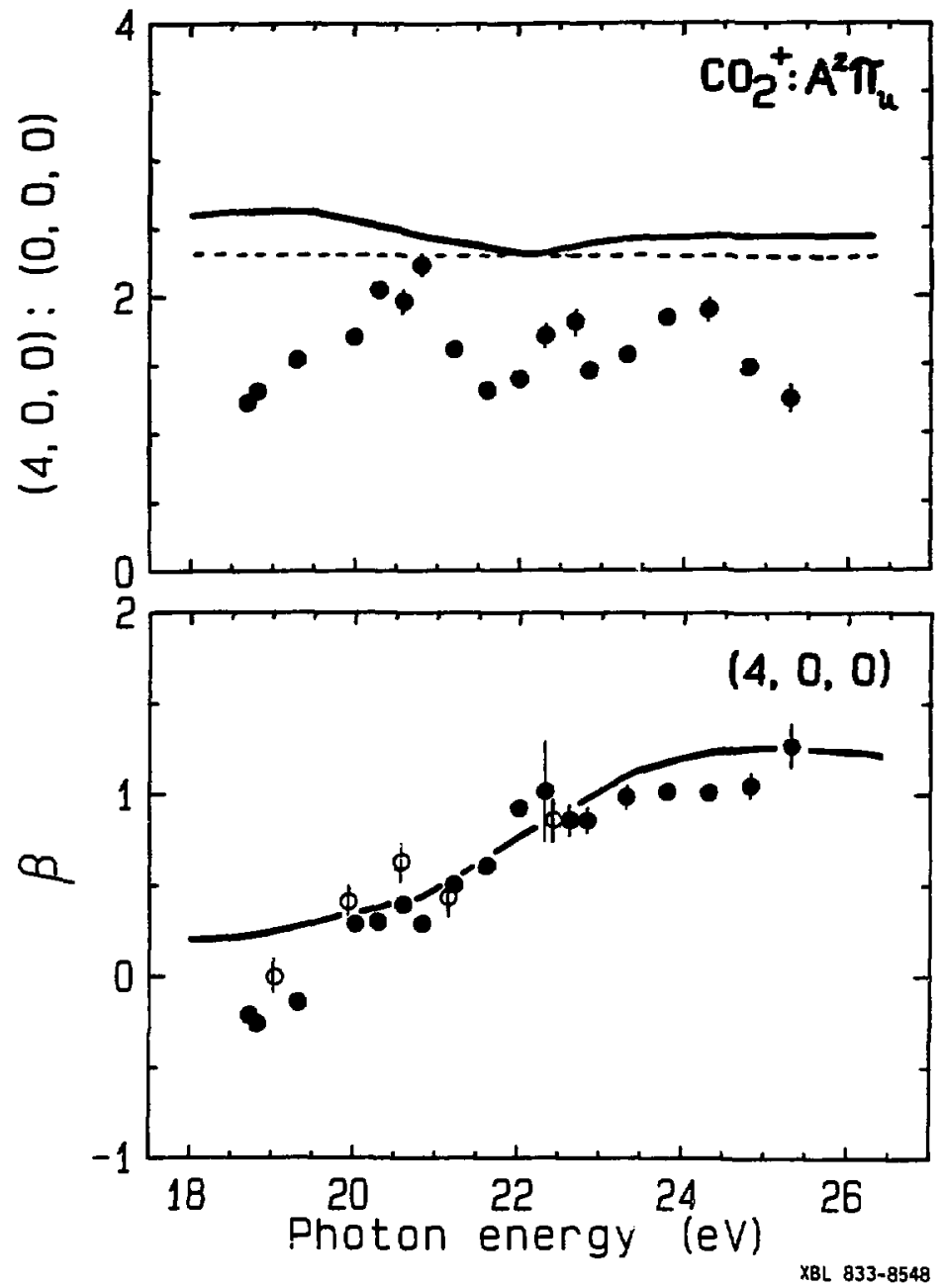

Fig. 7 


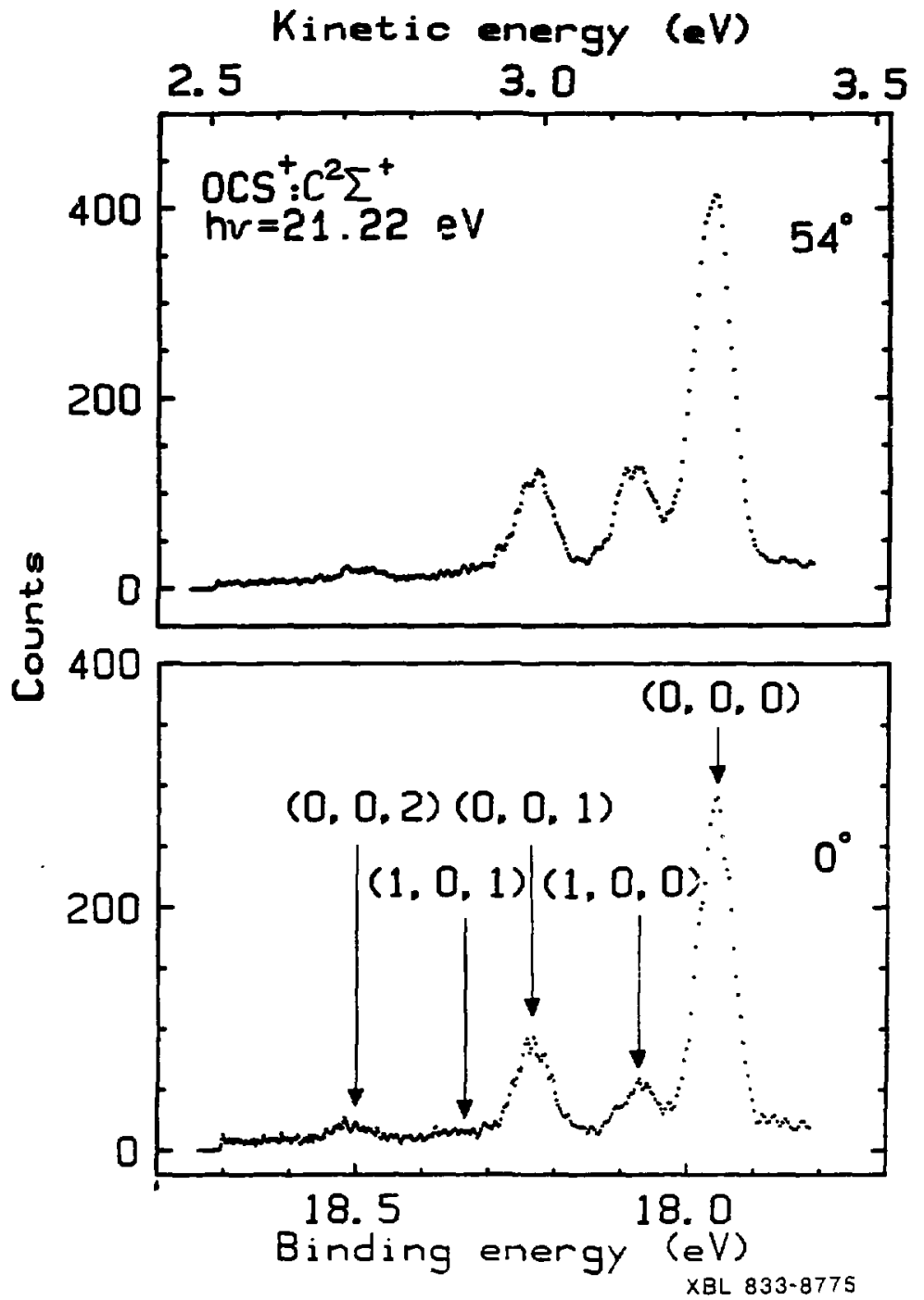

Fig. 8 
115

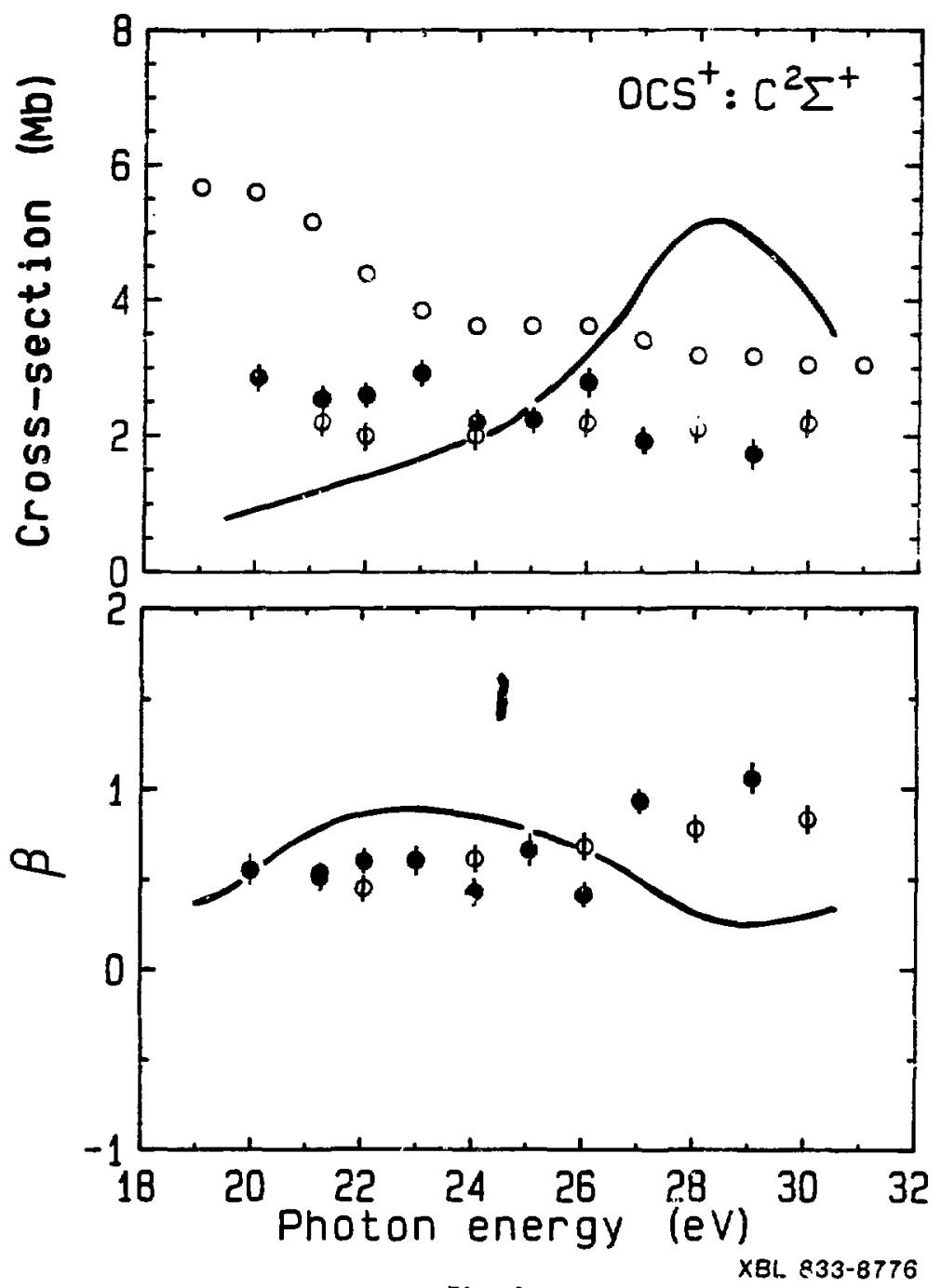

Fig. 9 
$1 ? 6$

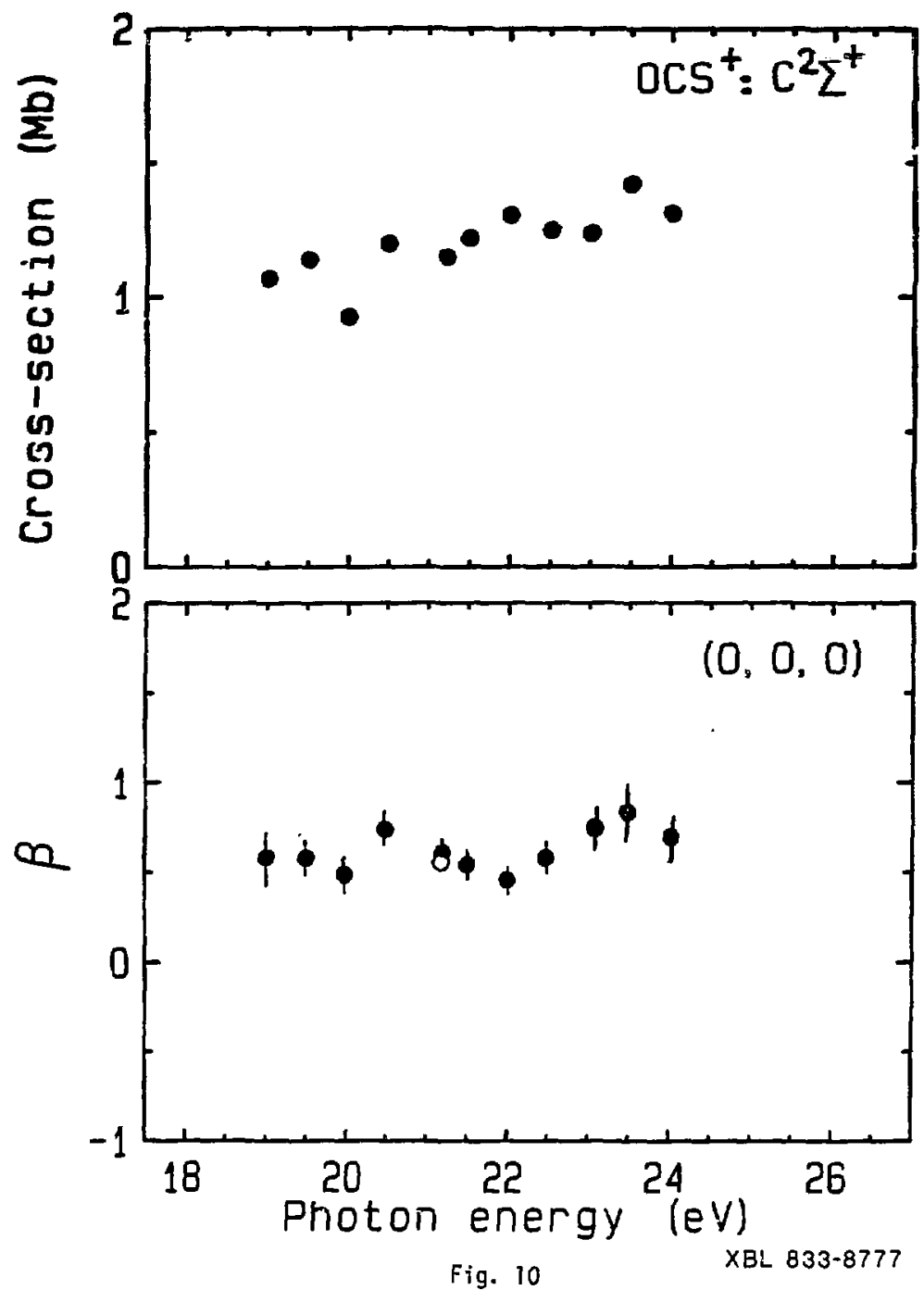



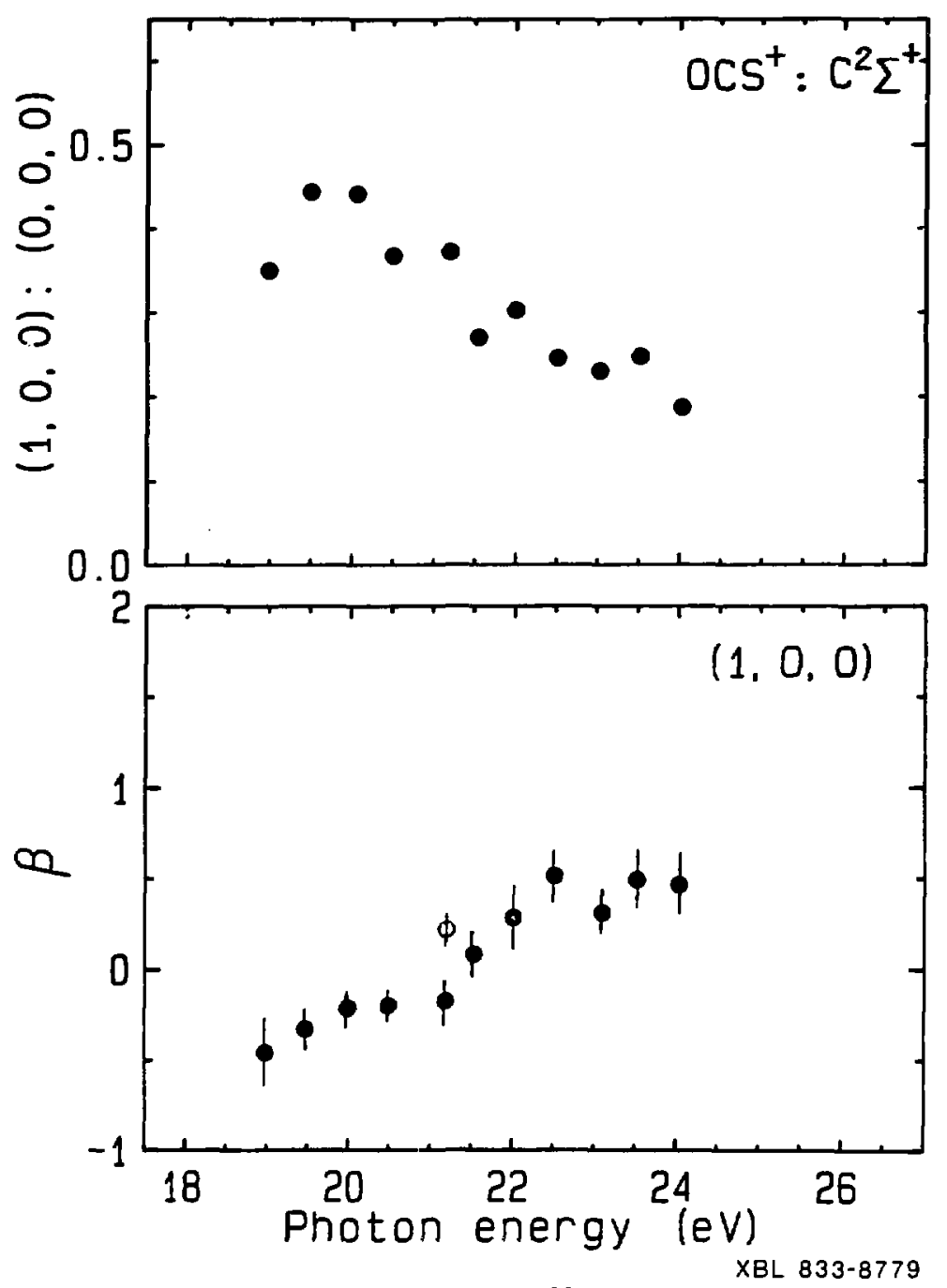

Fig. 11 
118
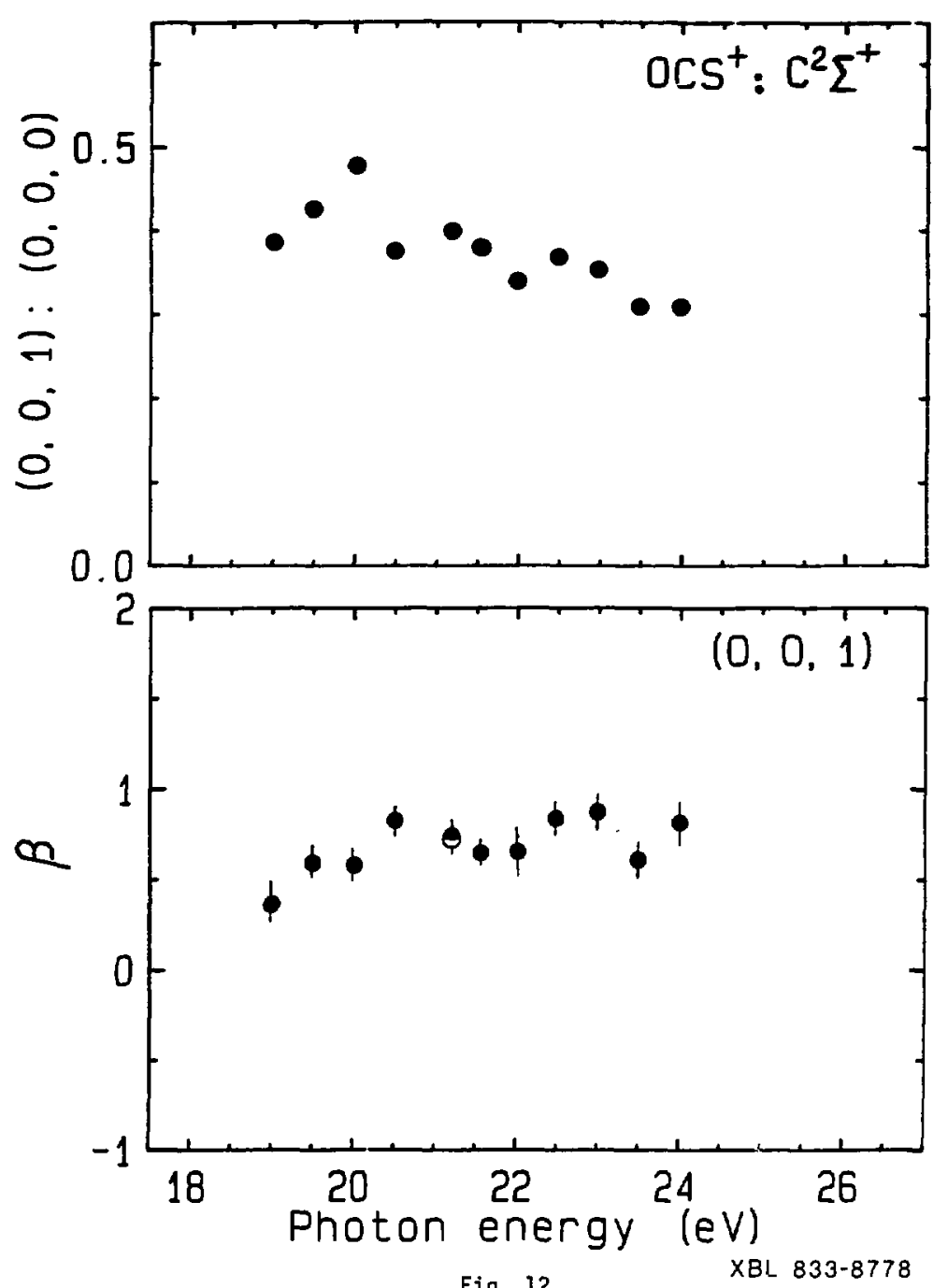

Fig. 12 


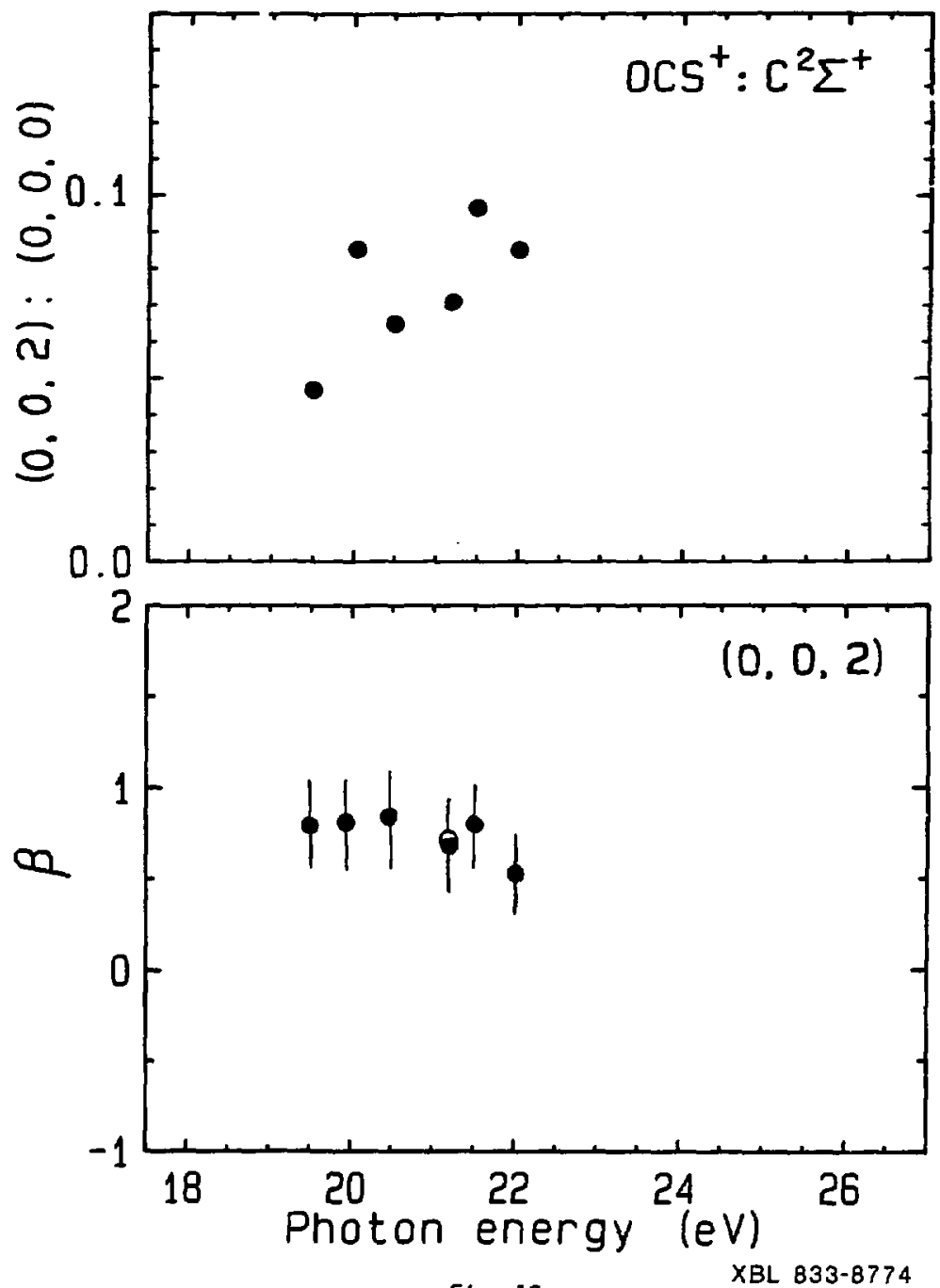

Fig. 13 


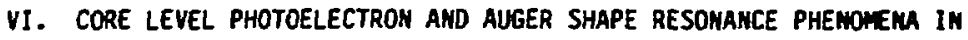 $\mathrm{CO}, \mathrm{CO}_{2}, \mathrm{CF}_{4}$, AND OCS}

\section{A. Introduction}

Direct photoexcitation of molecular core levels by variableenergy synchrotron radiation yields photoelectrons and Auger electrons. The differential cross section for ejecting electrons by either process should vary as

$$
\frac{d o(h v, \theta)}{d \Omega}=\frac{\sigma\left(h_{v}\right)}{4 \pi}\left[1+B(h v) P_{2}(\cos \theta)\right]
$$

provided that the initial photoionization mechanism has electric dipole character and the initial system is randomly oriented. 1 Here $P_{2}(\cos \theta)$ is the second Legendre polynomial, $\theta$ is the angle between the polarization direction of the exciting light and the electron propagation direction, and $h_{v}$ is the photon energy. The present work addresses experiments in which the electron yields are recorded as functions of electron kinetic energy. Most of the spectral features of interest are peaks in the electron yield associated with either photoelectrons or Auger electrons. For the former, the kinetic energy $\varepsilon$ of a peak corresponding to a given orbital of binding encigy $E_{B}$ is

\# C.M. Truesdale, S. Southworth, P.H. Kobrin, U.E. Becker, D.W. Lindle, H.G. Kerkhoff, P.A. Heimann, T.A. Ferrett, and D.A. Shirley, submitted to Phys. Rev. A (1983). 
given by $c$ - hu $-E_{B}$. For the latter, the kinetic energy of a given Auger channel is invariant with photon energy (post-collision interaction is negligible in this work). In both cases a complete description of the differential cross section can be given by the two functions $\sigma(h v)$ and $B(h v)$, which are called the "cross section" and "asymmetry parameter", respectively. They in turn can be determined by measuring $d \sigma($ liv, $\theta) / d \Omega$ at two known angles $\theta$. Given the close relationship between $h_{v}$ and $\varepsilon$, these parameters are often listed as $\sigma(\varepsilon)$ and $B(\varepsilon)$ for photoelectrons. Sometimes the notation $\sigma_{A}\left(h_{v}\right)$ and $B_{A}(h v)$ is used to denote properties of Auger transitions. In addition, Dill et al. ${ }^{2}$ have used the notation $B_{m}\left(h_{v}\right)$ to denote the alignment in the initial state of an Auger transition. The asymetry of each Auger transition is described by the product of an orientation parameter $B_{m}(h v)$ and a term dependent on the Auger decay process represented by the symbol $A$, where

$$
B_{A}\left(h_{v}\right)=B_{m}\left(h_{v}\right) A \text {. }
$$

Here the A parameter is independent of the excitation energy hu. For photon energies near the core-level binding energies of electrons in molecules, resonances in cross sections and associated variations in the asymmetry $B$ are expected. A certain class of these resonances is associated with the scattering of the outgoing photoelectron by the molecular potential in a quasi-bound state. These are termed "shape resonances", and are expected to constitute 
sensitive probes of the molecular potential. Although shape resonances for core-electron excitation in molecules have been the subiect of extensfve theoretical investigation, notably by Dehmer, Dill, and co-workers, ${ }^{2}$ they have not to our knowledge been observed by a direct experiment. He define a "direct" experiment as one in which both the energy and direction of the incoming photon and the outgoing electron are defined. In this paper, and an earlier preliminary report on CO, we describe the first direct measurements of the core-level shape resonances in the photoelecton cross section $\sigma(\varepsilon)$ and Auger cross section $\sigma_{A}(h v)$ for several molecules, and the first measurements of any $k$ ind on the photoelectron asymmetry $B(\varepsilon)$ and Auger asymmetry ${ }_{A}^{B}\left(h_{v}\right)$ of these same transitions.

Our results can be compared with related measurements in several cases. These include photoabsorption, ${ }^{3,4}$ electron energy loss, $5-10$ electron-ion coincidence, ${ }^{11}$ Auger yield, ${ }^{12}$ and valence-orbital photoemission. 13

Several theoretical predictions are available in addition to the MSMXa results. The Stieltjes-Tchebycheff Moment Theory (STMT) constructs ground state wavefunctions of Hartree-Fock quality from which static exchange potentials are approximated to account for the nonlocal properties of the core hole states. Pseudospectra are then produced that account for the frequencies and oscillator strengths of various transitions to discrete valence and continuum valence-like orbital channels. Padial et al. ${ }^{14-15}$ used the STMT formalism to calculate the partial cross sections for Cls and Ols photoemission 
from $\mathrm{CO}$ and $\mathrm{CO}_{2}$ - Recently a Hartree-Fock static exchange calculation by Lucchese and McKoy ${ }^{16}$ has yielded the $\mathrm{Cls}$ and 01 s cross sections $O(e)$ and asymmetry parameters $\mathrm{B}(\mathrm{e})$ for $\mathrm{CO}_{2}$. This calculation comes near to determining the correct ab-initio ground state and continuum wavefunctions.

Our spectrometer has been described previously. ${ }^{17}$ A double-angle-time-of-flight (TOF) spectrometer detects ejected electrons by means of microchannel plates. The gas samples are excited by photons delivered to Beam Line III-1 at the Stanford Synchrotron Radiation Laboratory. One detector is placed at $0^{\circ}$ and another is placed at $54.7^{\circ}$ relative to the photon polarization axis. Relative cross sections are determined from the electron intensities measured with the $54.7^{\circ}$ detector, and the asymmetry parameters are found from the ratio of signals measured at $0^{\circ}$ and $54.7^{\circ}$.

Cross sections and asymmetry parameters are corrected in a calibration procedure explained in Ref. 18. Briefly, the asymetry parameters are corrected for the relative efficiency of the two detectors as a function of the kinetic energy of detected electrons. Comparisons are made between accepted literature values for the as ymmetry parameters of $\mathrm{Ne} 2 \mathrm{~s}$ and Me $2 \mathrm{p}$ photoelectrons for photon energies of 50-300 eV and the ratio of their measured intensities at $0^{\circ}$ and $54.7^{\circ} .^{19}$ A small (up to several percent) unpolarized component of the synchrotron radiation and any small misalignment of the photon beam that intersects the gas sample in the interaction region would thereby be corrected for in the final determination of 
the asymmetry parameters. ${ }^{18}$ Likewise, our measured relative cross sections have been corrected for the (energy-dependent) transmission characteristics of the $54.7^{*}$ detector by a comparison with knom experimental cross sections and partial cross sections of He 2s and Me $2 p$ photoionization channeis. 19

Second-order light corrections for the $C(K V V)$ Auger cross section $\sigma_{A}(h v)$ and asymmetry parameter $B_{A}(h v)$ were negligible because of the small observed intensity for the second-order $\mathrm{Cls}$ photoelectron peak. The cross sections $\sigma(\varepsilon)$ and $\sigma_{A}(h v)$ for the 01 s and the $O(K V V)$ peaks measured with the second-order component of the light, and the first-order results, were scaled separately to the photoabsorption cross sections of Barrus and co-workers. ${ }^{3}$

Most spectra were collected for $1000 \mathrm{sec}$, the exceptions being those of the S2p and S(LVV) Auger peaks of OCS, for which the spectra were collected for $300 \mathrm{sec}$. In the $\mathrm{CO}, \mathrm{CO}_{2}, \mathrm{CF}_{4}$, and oCs carbon and oxygen $K$-shell experiments, an aluminum window (1500A thickness) isolated our chamber from the port of the ultra-high vacuum monochromator. A vitreous carbon window (1000A) was used for the ocs sulfur L-shell experiments.

Stanford Synchrotron Radiation Laboratory provided tunable radiation from the grazing-incidence "grasshopper" monochromator, 20 which was operated with a 1200 line/mm holographic ruled grating. Second-order light was used to measure the $01 \mathrm{~s}$ and 0 (KVV) electron Deaks while simultaneously measuring the $C 1 s$ and $C(K V V)$ electron peaks with first-order light for $\mathrm{CO}$ and $\mathrm{CO}_{2}$. 
Excitation spectra of discrete subthreshoid resonances (e.g., the $\sigma \rightarrow *^{\star}$ type) for $\mathrm{CO}, \mathrm{CO}_{2}$, and $\mathrm{OCS}$, and $\left(\mathrm{O} \rightarrow 3 \mathrm{sa}_{1}\right)$ for $\mathrm{CF}_{4}$ were used to calibrate the monochromator energy scale. The overall experimental resolution was determined by the observed widths of these resonances, which were larger than the natural line widths. The entrance and exit slit settings of the monochromator for the $\mathrm{CO}$ measurements (275 eV $\leq h_{v} \leq 315 \mathrm{eV}$ ) were $20 \mu$ and $50 \mu$, which yielded band passes of 0.5 and $2.0 \mathrm{eV}$ FWH. The $0.5 \mathrm{eV}$ resolution measurements (275 eV $\left.\leq h_{v} \leq 290 \mathrm{eV}\right)$ were performed over the Auger $* \rightarrow$ " discrete transition. Ouring the $\mathrm{CO}_{2}$ measurements $(270 \mathrm{eV} \leq \mathrm{hv}$ $\leq 340 \mathrm{eV}) 50 \mu$ slits were used. The S2p and S(LVV) Auger experiments (155 ev $\leq h_{v} \leq 190 \mathrm{eV}$ ) on oCs used $100 \mathrm{w}$ slits with a monochromator resolution of $1 \mathrm{eV}$, and the remaining studies used $100 \mathrm{p}$ slits which corresponds to a bandpass of $4 \mathrm{eV}$. In each case an additional 3 percent of the kinetic energy of the electrons arising from the geometry of our spectrometer must be factored in to calculate the overall resolution of our measurements. The oxygen measurements performed with first-order light for $C 0$ were carried out with a monochromator bandpass of $5 \mathrm{eV}$, and the second-order oxygen measurements for $\mathrm{CO}$ and $\mathrm{CO}_{2}$ were performed with $4.0 \mathrm{eV}$ monochromator resolution. For some spectra, the electrons were retarded for $14.4 \mathrm{~cm}$ of the total $28 \mathrm{~cm}$ path length of travel, before being detected by the microchannel plates, by means of retarding cages inside the flight tubes of our detectors. The resolution and separation of photoelectron and Auger peaks were significantly improved by this procedure. 
There are five sections to follow. The results and discussion for $\mathrm{CO}, \mathrm{CO}_{2}, \mathrm{CF}_{4}$, and OCS will be presented in Sections $\mathrm{B}-\mathrm{E}$, respectively, and conclusions will be presented in Section $F$.

\section{B. CO Results and Discussion}

Some of the $C O$ shape resonance results reported here were presented and discussed in an earlier report. ${ }^{21}$ He include them here for completeness, but refer the reader to that report for its complementary discussion.

The ground state electronic configuration of $\mathrm{CO}$ is $\left(1 \sigma^{2} 2 \sigma^{2} 3 \sigma^{2} 4 \sigma^{2} 1 \pi^{4} 5 \sigma^{2}\right) 1 \Sigma^{+}$. The lo orbital is basically 0ls-like $\left(E_{B}=\right.$ $541.2 \mathrm{eV})$, the $2 \sigma$ is $C l \mathrm{~s}\left(E_{B}=295.9 \mathrm{ev}^{22}\right)$, and the other four orbitals are the valence orbitals of $\mathrm{CO}$.

Two time-of-flight (TOF) spectra, converted to kinetic energy scales, are shown in Figs. 1 and 2 . The spectrum in Fig. 1 was collected with a retarding voltage of $150 \mathrm{eV}$. The $\left(5 \sigma^{-1}\right),\left(1 \pi^{-1}\right)$, and the $\left(4 \sigma^{-1}\right)$ photoelectron peaks, with binding energies of 14.9 , 17.6, and $20.5 \mathrm{eV}$, respectively, are unresolved, but the $\left(3 \sigma^{-1}\right)$ peak is distinct, with a binding energy of $35.4 \mathrm{eV}$. Peaks corresponding to the $C(K V V)$, the $C l s$ (from 2 nd order), the $01 s$ (from $3 r d$ order), and the O(KVV) peaks are also observed. A spectrum taken with no retarding potential is shown in Fig. 2. The Cls and 0ls peaks are evident, and the identities of the other features can be inferred from Fig. 1 . We note that the $C(K V W)$ Auger peaks have kinetic energies of $\sim 220$ 
to $273 \mathrm{eV}$. Using the notation of Moddeman et al. ${ }^{23}$ the 8-1 peak $\left(5 \sigma^{-1}, 1 \pi^{-1}\right.$ sinal state) with a kinetic energy of $-255 \mathrm{eV}$ is convoluted with $B-3\left(5 \sigma^{-2}\right.$ ) and the weak $B-2$ Auger band $\left(4 \sigma^{-1}\right.$, $\left.5 \sigma^{-1}\right) \cdot 24,25$ The other structures observed by Moddeman, et al. $(B-6$ to $B-10$, and $C-1$ to $C-3$ ) are grouped together into the peaks shown by the $B$ and $C$ labels. The Auger bands labeled by Moddeman et a1. as A-I to A-II (autoionization) have final kinetic energies corresponding to the kinetic energies of the ionized valence states, and cannot be unambiguously identified because of the presence of the yalence ionic channels. The oxygen Auger peaks have kinetic energies of -413 to $517 \mathrm{eV}$. The largest $0(\mathrm{KVV})$ peaks have been identified as the $B-5\left(1 \pi^{-2}\right)$ and $B-7\left(4 \sigma^{-1}, 1 \pi^{-1}\right)$ bands. 24 our peak at a kinetic energy of $\sim 495 \mathrm{eV}$ corresponds to B-5 convoluted with B-7 (shake-up) and B-4, and the shoulder at $-500 \mathrm{eV}$ is the $8-1\left(5 \sigma^{-1}\right.$, $1 \pi^{-1}$ ) band. ${ }^{24}$ The results of ab-initio molecular Auger calculations by Agren ${ }^{25}$ suggest that the large C(KVV) Auger peaks arise from the vacancies in the $5 \sigma$ orbital, and that vacancies in the $3 \sigma, 4 \sigma$, and $1 \pi$ orbitals dominate the O(KVV) Auger spectrum for $\mathrm{CO}$. Asymmetry parameters for the C(KVV) Auger electrons of $\mathrm{CO}$ are shown in Fig. 3. The asymmetry parameters are set out in Table 1 . Spectra excited by photons of energies near the Cls edge energy contained valence orbital photoe?. .ron peaks that overlapped with, and were unresolvable from, the Auger peaks. However, extrapolation from spectra taken with photon energies too low to excite the $\mathrm{Cls}$ discrete resonances enabled us to estimate with some confidence the 
effects of the valence photoelectrons on the Auger partial cross sections. In some cases a partial deconvolution of these peaxs was attempted, but in most cases the peaks arising from higher order light and the first three valence photoelectron peaks could be on $7 y$ partially deconvoluted from the $C(K V V)$ peaks, and some $\left(3 \sigma^{-1}\right)$ component is unavoidably still included in the $C(K V V)$ results. The effective asymetry parameter $B_{T}\left(h_{v}\right)$ for the sum of the $C(K V v)$, the valence states, and the small second order C1s peak is also given in Table I. Of course the tiransitions represented by $B_{T}\left(h_{v}\right)$ vary with hv. For example, for $h_{v}<280 \mathrm{eV},{ }_{\mathrm{T}}\left(h_{\nu}\right)$ corresponds to the effective as ymmetry parameter for the sum of the valence states, because at these photon energies the CikVV) Auger transitions do not exist.

The cross section $\sigma_{A}(h v)$ for the $2 \sigma \rightarrow 2 \pi\left(\pi^{*}\right)$ resonance, at $287.3 \mathrm{eV}$ photon energy, $5,6,10,11$ has been scaled to the integrated oscil'itor strength over this resonance given by Tronc et al. 5 This discrete resonance is over an order of magnitude more intense than the continuum excitations, which lie above the Cls binding energy of 295.9 eV. For phot: $n$ energies above the discrete resonance, the $C(K V V)$ curve was scaled by a constant factor, to agree with the $\mathrm{Cls}$ phctoelectron cross section at $315 \mathrm{eV}$, which was normalized to the electron-ion coincidence measurements, shown by the dashed curve. The continuum region shows structure arising from the $\mathrm{Cls}$ photoelectron shape resonance, as discussed before. In normalizing the Auger yield to the $\mathrm{Cls}$ cross section wie have neglected the fluorescence yield, 
which could be accounted for in a more precise study.

The asymmetry parameter $B_{A}(h v)$ for the $C(K V V)$ char.nel in $C 0$ is nearly zero over the entire region where measurements were taken. The exception is near $295 \mathrm{eV}$. The published electron energy loss measurements ${ }^{10}$ showed a strong discrete resonance near this energy. Our data indicate that in this resonance the excited $\mathrm{CO}$ molecule is strongly oriented, leading to an asymmetry in the C(KVV) Auger channel. No further interpretation is warranted, because the nature of the discrete resenance at $295 \mathrm{eV}$ is not well established. Turning now to the $287.3 \mathrm{eV}$ resonanca, the orientation parameter for the excited state following the $\sigma \rightarrow \pi^{*}$ transition is predicted to have a value of $-1 .^{2}$ This prediction has essentially been confirmed by Stöhr et al. ${ }^{12}$ for $C O$ adsorbed and oriented on a surface. It seems inescapable that $\mathrm{CO}^{*}$ is strongly oriented in the $\pi$ resonance excited state. There are two possible ways to reconcile this orientation with our observations that the kinetic energy-integrated $C$ (KVV) asymmetry parameter $B_{A}\left(h_{v}\right)$ is essentially zero at $h_{v}=287.3 \mathrm{eV}$. First, the $A_{j}$ factors for the various Auger transitions of fractional strength $f_{j}$ could have values that wouid average out the Auger asymmetry parameter $B_{A}\left(h_{v}\right)$ to zero, according to

$$
\overline{\theta_{A}}\left(h_{v}\right)=B_{m}\left(h_{v}\right) \sum_{j} A_{j} f_{j}\left(h_{v}\right) \quad\left(\frac{-1}{2} \leq A_{j} \leq 1\right)
$$

This can also explain the discrepancy between the calculated orientation parameter $B_{m}\left(h_{v}\right)$ and our asymetry parametar $B_{A}\left(h_{v}\right)$ 
values for the $C(K V V)$ channel above the Cls threshold at $295.9 \mathrm{eV}$. Hell-resolved Auger spectra will have to be measured to test this possibility. Dur own attempts to obtain such spectra will be described below, after we address the alternative explanation for a near-zero as ymmetry.

The above idea that the Auger asymmetry $B_{A}$ is near zero because of cancellation of asymetries strains our credulity, especialiy if it must be invoked twice; i.e., for the "resonance and again for the continuom states. An attractive alternative explanation for the near-zero $B$ of the " resonance is the "spectator" model, in which the excited electron retains the orientation information in a $\pi^{*}$ orbital but is not significantly involved in subsequent Auger decays. The molecular "core" would then behave somewhat like an atom with a K-shell hole and exhibit no asymetry in its Auger decay. This model was discussed earlier. ${ }^{21}$

In an attempt to test the first explanation, we used selected retarding potentials to study the kinetic energy distribution of the Auger electrons for photon energies above and below the $\mathrm{Cls}$ threshold at $295.9 \mathrm{eV}$. Two typical spectra, excited by photons on resonance at $287.8 \mathrm{eV}$ and above threshold at $296.8 \mathrm{eV}$, are shown in Fig. 4. Retarding potentials of $100 \mathrm{~V}$ and $150 \mathrm{~V}$ were applied to collect the $287.8 \mathrm{eV}$ and $296.8 \mathrm{eV}$ spectra, respectively. The large $\mathrm{C}(\mathrm{KVV})$ peak in the $287.8 \mathrm{eV}$ spectrum, which corresponds to the $\mathrm{B}-1$ band $\left(5 \sigma^{-1}, 1 \pi^{-1}\right)$ in the $296.8 \mathrm{eV}$ spectrum, has a kinetic energy of around $12.5 \mathrm{eV}$ higher than its counterpart, because the initially excited electron is 
st111 present. An electron-electron coincidence measurenent by T.D. Thomas et al. ${ }^{26}$ has shown a similar shift between high resolution C(KVV) Auger spectra for $\mathrm{CO}$ and for comparable measurenents by Ungier et a). 27 for the $N(\mathrm{KVV})$ Auger of $N_{2}$ spectra with and without the excited electron present. Because of the low resolution of our spectra we could only confirm tnat the mean energies and overall shapes of the spectra were different. He could not establish whether or not the asymetry varies with kinetic energy across a spectrum =

The results for the $\mathrm{Cls}$ photoelectron channel are presented in Fig 5. Our relative cross sections were scaled to the electron-ion coincidence measurements of Kay et al., 11 shown by the open circles. The CIs cross section shows a weak shape resonance maximum centered around $306 \mathrm{eV}$, in good agreement with the results of Kay et a). ${ }^{11}$ and with predictions of the STMT calculation, ${ }^{14}$ shown by the solid curve. In the STMT work two major subchannel excitations were invoked to describe the $20 \mathrm{~K}$-shell excitations. Those were the $20 \rightarrow k_{0}$ and the $20 \rightarrow k \pi$ continuum transitions. The $\ell=3$ partial wave in the $0 \rightarrow k_{0}(\varepsilon f)$ transition has been suggested as being responsible for the o shape resonance. ${ }^{28-31}$ Also shown in Fig. 5 is a dashed curve representing the MSMXa prediction. ${ }^{31}$ This curve shows a maximum at the right energy, but its width exceeds the experimental value and its contrast ratio is about twice the experimental result. The asymmetry parameter $B(\varepsilon)$ for the $C l s$ photoelectron confirms the existence of a shape resonance with a weak minimum at $303 \mathrm{eV}$. The variation of the measured $\theta(c)$ falls between the predictions of the 
localized-hole MSMXa calculations of Dill et al. ${ }^{30}$, shown by the solid curve, and Grimm, ${ }^{32}$ shown by the dashed curve. The overall shapes of the calculated $B(c)$ curves are in very good agreenent with the present results, except for predicting a contrast ratio larger than is observed.

The cross section and asymetry parameter of the unresolved peak, which included the $\mathrm{X}, \mathrm{A}$, and $\mathrm{B}$ states of $\mathrm{CO}^{+}$derived by ionization of $5 \sigma, 1 \pi$, and $4 \sigma$ electrons, and the $C l s$ peak in second order, was also derived from our data. The cross section showed littie variation with photon energy in the range $h v=270-315 \mathrm{eV}$, and $\mathrm{B}$ ranged between 1.5 and 2. The data showed a lot of scatter because of the difficulty of deconvoluting these peaks from the Auger structure, but their overall behavior assured us that the curves derived for the Auger peaks were entirely due to the behaviour of those peaks.

The Cls(2nd order) peak could be deconvoluted in our spectra for $h_{v}=308-314 \mathrm{eV}$; i.e., for 2nd order photon energies raiging from 616 to $628 \mathrm{eV}$. In those five spectra the asymmetry parameter $\theta(\varepsilon)$ was determined, the mean value being $\mathrm{B}=2.03 \neq 0.11$. Of course $\mathrm{B}=2$ is expected for an atomic $n s \rightarrow e p$ transition. In the high kinetic energy regime it may be plausible to treat the Cls excitations in molecular $\mathrm{CO}$ in an atomic model, because the scattering dynamics should not include resonances and the outgoing electron would have little interaction with the molecular potential.

The oxygen Auger results are dispayed in Fig. 6. The data are incomblete because the oxygen edge was a low-priority secondary 
objective of this study, as a consequence of the poor performance of the monochromator at this energy. Our results were derived by using both first-order and second-order light. Ho data were taken below $h v=540 \mathrm{eV}$ in either order, and the range hv $=550-570$ el was largely missed, thereby precluding a definitive study of shape resonance phenomena. The results are nonetheless of some interest.

The $O(K V V)$ cross section closely mimics the 015 cross section (to be discussed later), as it should. The O(KVV) asymmetry parameter was easy to work up because the peak fell at a high kinetic energy and was well separated from other features. Throughout the range $h v=560-630$ eV the data lie in the range $B=-0.1$ to 0.3 with no real trends, and a horizontal straight line fits the data within their statistical accuracy, yielding the value $s=0.10 \neq 0.02$.

Our six points near threshold, in the range hv $=545-555 \mathrm{eV}$, show a larger $\mathrm{s}$, in the range $0.3-0.5$. It seems probable that these points provide the first evidence for an Auger shape resonance, as predicted by $0 i 11$ et al. ${ }^{2}$ Their calculated curve for the orientation parameter $\theta_{m}$ is shown in Fig 7. Clearly more work is needed on this question.

The results for the 01s peak are presented in fig. 7. There is general agreement among our cross section $\sigma(\varepsilon)$ data, photoabsorption measurements, ${ }^{3}$ and the STMT calculation of Padial et al. 14 Our measurements based on first-order and second-order light, after being corrected for transmission efficiency, were scaled to the photoabsorption data at $545 \mathrm{eV}$, and $562 \mathrm{eV}$, respectively, because of a 
small overlap in energles for the two sets of measurements. The 015 cross section o(c) clearly shows a shape resonance near $550 \mathrm{eV}$.

The dearth of measurements between 550 and $570 \mathrm{eV}$ prevents our observing the minimum in the 015 asymetry $B(c)$ predicted by the localized-hole MSMXe calculations of Grimn ${ }^{32}$ and Dill et al. ${ }^{30}$. However, we find that $B(c)$ increased rapidly from 0.7 at hv $=545 \mathrm{sv}$ toward an asymptotic value above 1.5 by $h_{v}=570 \mathrm{eV}$. Whether there is more structure in the range hy $=550-570 \mathrm{gV}$ is unknown. At higher energies our data appear to approach an asymptotic value of $\beta=1.6 \pm$ 0.1 . The MSMXe calculations 30,32 show good agreement with this result, approaching an asymptote this high or higher. Similar behavior was observed both experimentally and theoretically for the Cls as ymmetry.

Shake-up structure is observed near the 01s peak. A TOF spectrun taken with second-order light at $h_{v}=630 \mathrm{eV}$, converted to an energy scale, is shown in Fig. 8. The shaks-up structure labeled "S" is probably the $1 \pi \rightarrow 2 \pi$ shake-up transition. We find that this structure is present at $13.0 * 3.0$ percent of the $01 \mathrm{~s}$ main-line intensity. Carlson et al. ${ }^{33}$ reported that this state has -10 percent of the $01 \mathrm{~s}$ intensity. Aarons et al, ${ }^{34}$ performed an unrestricted Hartree-Fock (UHF) calculation to assign shake-up thresholds and intensities in the high-energy 1 imit. They predicted that the $1 \pi \rightarrow 2 \pi$ shake-up peak should lie $16 \mathrm{eV}$ above the $01 \mathrm{~s}$ peak and would have 15.4 percent of the $01 s$ intensity. The agreement between this calculation and our measurements is very good. 


\section{C. $\mathrm{CO}_{2}$ Results and Discussion}

The ground state electronic configuration of $\mathrm{CO}_{2}$ can be written $1 \sigma_{g}^{2} 1 \sigma_{u}^{2} 2 \sigma_{g}^{2} 3 \sigma_{g}^{2} 2 \sigma_{u}^{2} 4 \sigma_{g}^{2} 3 \sigma_{u}^{2} 1 \pi_{u}^{4} 1 q_{g}^{4} \quad 1 \varepsilon_{g}^{+}$. The $1 \sigma_{g}$ and $1 \sigma_{u}$ orbitals (unresolved in this work) are linear combinations of atomic 01s orbitals with an average birding energy of $541.2 \mathrm{eV}$. The $2 \sigma_{\mathrm{g}}$ orbital is basically a Cls atomic orbital with a binding energy of $297.5 \mathrm{eV}$. The remaining molecular orbitals have binding energies below $50 \mathrm{eV}$, and were not studied in this work. A spectrum is shown in Fig. 9. The Cls photoelectron and $\mathrm{C}(\mathrm{KVV})$ Auger cross section results were scaled at 305 and $302 \mathrm{eV}$, respectively, to the Cls partial cross sections derived by the electron energy loss studies of Wight and Brion, ${ }^{8}$ which were themselves scaled to agree at $300 \mathrm{eV}$ with the STMT calculations of Padial et al. ${ }^{15}$. As in $\mathrm{CO}$, all of the $01 \mathrm{~s}$ and $\mathrm{O}(\mathrm{KVV})$ measurements in $\mathrm{CO}_{2}$ were taken with the second-order light, and our relative partial cross sections were scaled to the photoabsorption measurements of Barrus et al. ${ }^{3}$

Results for the C(KVV) asymmetry parameter are presented in Table II. The $C(K W)$ peak is convoluted with the valence photoelectron peaks: a partial deconvolution was achieved. The Auger asymmetries for the $C(K V V)$ and $O(K V V)$ channels of $\mathrm{CO}_{2}$ are effective values integrated over the unresolved KVV Auger channels, similar to the results for the Auger peaks in $C O$. As for the $C O C(K V V) B_{A}(h v)$ results, the effective asymmetry parameter in $\mathrm{CO}_{2}$ for the sum of the $C(K V V)$ peak, the valence states, and the second-order Cls peak, 
$B_{T}(h v)$, is also found in Table II. To our knowledge, there are no published integrated cross sections over the discrete $2 \sigma_{g} \rightarrow 2 \pi_{u}\left(\pi^{*}\right)$ transition against which we can normalize our Auger cross sections.

The time-of-flight spectrum of $\mathrm{CO}_{2}$ in $\mathrm{Fig}$. 9 has been converted to a linear kinetic energy scale, with the various peaks identified in the Figure. The dominant $C(K V V)$ peaks have been assigned as having mainly $\left(1 \pi_{u}^{-2}\right)$ and $\left(4_{\sigma}^{-1}, 1 x_{g}^{-1}\right)$ final states. ${ }^{25}$ Some of the $0(k v V)$ peaks of $\mathrm{CO}_{2}$ still do not have unambiguous assignments, but the $1 \pi \mathrm{g}$ hole states should account for most of the high kinetic energy Auger peaks. 25

The derived parameters for $\mathrm{CO}_{2}$ are plotted in Figures 10-13. The format remains the same, where filled circles represent the present experimenta? measurements. The open circles with the $\mathrm{Cls}$ o(e) results and the dashed curve with $C$ (KVV) Auger cross section $\sigma_{A}(h v)$ are the electron energy loss measurements of Wight and Brion, ${ }^{8}$ and the open circles shown with the $\sigma_{A}(h v)$ for the $O(K V V)$ channel denote photoabsorption measurements. ${ }^{3}$ The solid curves presented with the $\sigma(\varepsilon)$ and $\sigma_{A}(h v)$ data are the STMT results, ${ }^{15}$ and the dashed curves represent the HF static exhange calculations. 16 For the $B(\varepsilon)$ curves, the solid curve represents the HF static exchange results, the dotted curve represents the localized MSM calculation of Grimm, 32 and the dashed eurve represents the unlocalized-hole MSMXa calculations of Grimm. 32 
In Fig. 10 the $\mathrm{CO}_{2} \mathrm{C}$ (KVV) Auger cross section $\sigma_{A}(\mathrm{~h} v)$ shows a discrete resonance transition $\left[2 \sigma_{g} \rightarrow 2 \pi_{u}\left(\pi^{*}\right)\right]$ centered near 290 el and the broad shape resonance $\left[2 \sigma_{g} \rightarrow 4 \sigma_{u}\left(\sigma^{*}\right)\right]$ with its maximum near $310 \mathrm{eV}$. Our $C(\mathrm{KVV})$ Auger cross section $\sigma_{A}\left(h_{v}\right)$ results were scaled to the electron energy loss results at $302 \mathrm{eV}$.

The $\mathrm{CO}_{2} \mathrm{C}(\mathrm{KVV})$ Auger as ymmetry parameter $\mathrm{B}_{A}(\mathrm{hv})$ is also shown in Fig. 10. At the $2 \pi_{U}\left(\pi^{*}\right)$ resonance $B_{A}\left(h_{v}\right)$ is small, similar to the result for the $C(K V V)$ channel of $C O$ at the $2 \pi\left(\pi^{\star}\right)$ resonance, but apparently nonzero. As in $\mathrm{CO}$, the remainder of the asymetry points must be interpreted carefully. The highest asymetry values fall at energies for which the Auger peak intensity is very weak; i.e., $h_{v}=296,300$, and $>330 \mathrm{eV}$. These high values cannot be interpreted with any confidence, but probably arise from other channels, e.g., valence orbitals. In the range $300<\mathrm{hv}<325 \mathrm{eV}$, ${ }_{A}{ }_{A}\left(h_{y}\right)$ shows scatter well outside of statistics, but again (as for C0) appears to be slightly positive. The discussion given for the $c 0$ $C(K V V)$ Auger asymetry would also be appropriate for $\mathrm{CO}_{2}$.

The results for the Cls photoelectron channel are presented in Fig. 11. The cross section of(c) for the $\mathrm{Cls}$ peak of $\mathrm{CO}_{2}$ was scaled in the same way as was the $C(K V V)$ cross section. The o shape resonance peaking at $312 \mathrm{eV}$ is more evident than that of $\mathrm{CO}$. Both of the theoretical models (HF static exchange and MSMXa) are fairly accurate in determining the shape of the peak in the cross sectiori, but the energy of the broad shape resonance is calculated to lie 5-6 eV closer to threshold; i.e., at c $-8 \mathrm{eV}$ rather than the experimental 


\section{8}

value of $c-14 \mathrm{eV}$. Wight and Brion ${ }^{8}$ suggested that the structure present at $301 \mathrm{eV}$ is attributable to $1 \pi_{u}$ shakeup $\left(1 \pi_{u} \rightarrow 2 \pi_{u}\right)$. Both theories miss this structure.

The asymetry $\mathrm{B}(\mathrm{c})$ of the $\mathrm{Cls}$ peak in $\mathrm{CO}_{2}$ is also more dramatic than for $\mathrm{CO}$, appearing as a broad minimum centered around $318 \mathrm{eV}$. The HF static exchange calculation ${ }^{15}$ (solid curve) is in better agreement in predicting the overall shape of $B(E)$ than is the unlocalized-hole MSMXo calculation, ${ }^{32}$ shown by the dashed curve. Nonetheless, the unlocalized-hole MSMXa predicts the energy position for the shape resonance minimum very close to our measurements. The localized-hole MSM calculation of $B(c)$ (dotted curve) for the Cls channel given by Grimm 32 predicts the shape resonance too close to threshold. Grimm suggested the Cls shape resonance is attributable to the $\ell=2,3$ channels in the $2 \sigma_{g} \rightarrow$ $4 \sigma_{u}\left(\sigma^{*}\right)$ continuum transition.

The $O(K V V)$ results are presented in Fig. 12. The cross section of the $O(K V V)$ channel should be directly related to the 0ls partial cross section when the photon energy used is above the 0ls threshold. We shall use the Auger yield $\sigma_{A}\left(h_{v}\right)$ for the $0(\mathrm{KVV})$ channel of $\mathrm{CO}_{2}$ to make comparisons to previous 01s cross section measurements and $01 \mathrm{~s}$ theoretical models. The partial cross sections for the $\mathrm{CO}_{2} \mathrm{O}(\mathrm{KVV})$ were scaled to the 0ls photoabsorption measurements of Barrus et al. ${ }^{3}$ at 555 eV to yield absolute partial cross sections. The Auger yield $\tau_{A}\left(h_{v}\right)$ for the $O(K V V)$ peak is then in excellent agreement in its energy dependence with the photoabsorption measurments. Barrus et al. stated that they observed a weak structure at $-580 \mathrm{eV}$ in their absorption curve, 
which was proposed to be related to shake-up structure. There also seems to be a weak structure present in our yield data. The $\sigma_{A}\left(h_{v}\right)$ data are in total agreenent with the photoabsorption results. Both theoretical studies predict the energy of the $\mathrm{Cls}$ shape resonance to be near $560 \mathrm{eV}$, but the STMT calculation is in closer quantitative agreement with the experiment in predicting the shape of the cross section.

The measured $O(K V V)$ asymmetry parameter $B_{A}(h V)$, shown by the filled circles in Fig. 12, starts at a value near 0.3 at $550 \mathrm{eV}$, decreases to near zero at $575 \mathrm{eV}$, has a weak maximum at near $580 \mathrm{eV}$, and remains close to 0.1 from $600 \mathrm{eV}$ to $680 \mathrm{eV}$. The possible structure near $580 \mathrm{eV}$ corresponds to the same feature observed in the yield $\sigma_{A}(h v)$, and may be a result of shake-up. ${ }^{3}$

The asymmetry parameter $B(\varepsilon)$ for the $01 s$ channel is shown in Fig. 13. It rises from 0.7 at $550 \mathrm{eV}$, to -1.3 at $555 \mathrm{eV}$, continues to increase to 1.5, and remains at this value from 560 to $680 \mathrm{eV}$. Our results are sparse at the low energies (the region where both the MF static exchange and the localized-hole MSMXa calculations predict minima in $B(\varepsilon))$ and the monochromator resolution was poor in this region. Therefore, we cannot infer anything about a possible minimum for the asymetry parameter.

After expanding the spectra around the ols peak, Ols satellite structure was again observed. In Fig. 14 we present TOF spectra converted to an energy scale for a spectrum collected with the second-order light with an energy of $630 \mathrm{ev}$. The largest $01 \mathrm{~s}$ shakeup 
peak is located at about $16 \mathrm{eV}$ above the 01s threshold. Allan et al. 35 have attempted an assignment of the total 01 s shakeup structure of $\mathrm{CO}_{2}$. They suggest that the two shake-up peaks at around $13.8 \mathrm{eV}$ and $16.0 \mathrm{eV}$ (referenced to the $01 \mathrm{~s}$ main-peak) may arise from transitions between the excitations $4 \sigma_{g} \rightarrow 5 \sigma_{g}(14.5 \mathrm{eV})$, $1 \pi_{u}>2 \pi_{u}(15.2 \mathrm{eV})$, and the $3 \sigma_{u} \rightarrow 4 \sigma_{u}(15.5 \mathrm{eV})$. The branching ratio for the total 015 shake up intensity to the $01 \mathrm{~s}$ main-line intensity is tabulated in Table III. Theoretical calculations ${ }^{34}$ have indicated that -20 percent of the intensity of the 01s main line is borrowed to produce the 01s satellite structures. The present average value for the branching ratio of the satellite peaks from $\sim 11$ to $22 \mathrm{eV}$ above the $01 \mathrm{~s}$ binding energy for photon energies between 592 and $632 \mathrm{eV}$ is about 20 percent. Allan and co-workers ${ }^{35}$ found that when $\mathrm{CO}_{2}$ was excited by $\mathrm{Mg}$ Ka radiation (1254.6 eV) the sum of the 01s shake-up peaks accounted for about 17.5 percent of the intensity of the $01 \mathrm{~s}$ photoelectron peak.

\section{D. $\mathrm{CF}_{4}$ Results and Discussion}

The ground state electronic configuration of this tetrahedral malecule can be written: $1 t_{2}^{6} 1 a_{1}^{2} 2 a_{1}^{2} 3 a_{1}^{2} 2 t_{2}^{6} 4 a_{1}^{2} 3 t_{2}^{6} i e^{4} 4 t_{2}^{6} 1 t_{1}^{6},{ }_{A_{1}} \cdot 36$ The $1 t_{2}$ and $l a_{1}$ molecular orbitals are described by a linear combination of $F 1 s$ atomic orbitals. The $2 a_{1}$ molecular orbital is formed almost entirely from a $\mathrm{Cls}$ atomic orbital with a binding energy of $301.8 \mathrm{eV}$. An electron spectrum of $\mathrm{CF}_{4}$ excited by a photon beam of nominal (first-order) energy $318.8 \mathrm{eV}$ is shown in Fig. 15. The 
features present are the Cls peak, the C(KVV) peak, the valence photoionization channels, and the $F(K V V)$ Auger peaks which arise from third-order light. We shall discuss only the $\mathrm{Cls}$ and $\mathrm{C}(\mathrm{KVV})$ peaks, which were observed in spectra taken at photon energies between $280 \mathrm{eV}$ and $350 \mathrm{ev}$.

A discrete resonance occurs at $298 \mathrm{eV}$, which has been assigned as arising mostly from the $2 a_{1} \rightarrow 3 s_{1}$ Rydberg-type excitation, with small contributions from $3 p$ and $3 d$ Rydberg orbitals. ${ }^{9}$ The derived parameters for the $\mathrm{Cls}$ and $\mathrm{C}(\mathrm{KVV})$ pedks of $\mathrm{CF}_{4}$ are plotted in fig. 16 and fig. 17. In these figures, our derived partial cross sections are compared to the electron energy loss measurements of Wight and Brion ${ }^{9}$, shown as open circles, and the photoabsorption results of Bachrach et al., 4 illustrated by the solid lines. There are no theoretical or other experimental $B(E)$ results for the $\mathrm{Cls}$ and $\mathrm{C}(\mathrm{KVV})$ channels of $\mathrm{CF}_{4}$. A salid line is orawn through our data to show their trends more clearly.

The measured $C(K V V)$ Auger cross sections $o_{A}\left(h_{v}\right)$ were scaled to the results of Bachrach et al. ${ }^{4}$ at $302 \mathrm{eV}$. To account for the valence contributions to their results, their cross section below the discrete resonance energy (298 eV) was assumed to be the valence orbital contribution to the absorption curve and was subtracted prior to scaling our results. There is good agreement between the photoabsorption study and the present work. Differences observed between the two could be a result of our using a $4 \mathrm{eV}$ bandpass in the monochromator, or the flux may not be well enough determined for the 
1200 line/mm grating at the carbon edge in the $\mathrm{CF}_{4}$ and OCS carbon $K$-shell experiments, which were performed 3 months after the $\mathrm{CO}$ and $\mathrm{CO}_{2}$ experiments. The incident photon flux during the $\mathrm{CF}_{4}$ and $\mathrm{OCS}$ experiments showed a stronger decrease at the carbon edge than observed for the $\mathrm{CO}$ and $\mathrm{CO}_{2}$ measurements for which a new grating was used. The gratings become contaminated with carbon from hydrocarbon residuals in the vacuum of the SSRL beam lines. The intensity ratio of the discrete resonance to the continuum $\sigma$ shape resonance in $\mathrm{CF}_{4}$ lies between those observed in $\mathrm{CO}_{0}$ and $\mathrm{CO}_{2}$.

The C(KVV) Auger asymmetry parameter $\mathrm{B}_{A}(\mathrm{hv})$ for $\mathrm{CF}_{4}$ is also shown in Fig. 16. The value of $B_{A}\left(h_{v}\right)$ at the discrete resonance $(298 \mathrm{eV})$ is near zero, as was the case for the C(KVV) peak in $\mathrm{CU}$ and $\mathrm{CO}_{2}$. ine apparent $C(\mathrm{KVV})$ Auger asymetry $B_{A}\left(h_{v}\right)$ for photon energies above the Cls threshold in $\mathrm{CF}_{4}$ lies in the range 0.2-0.3. It shows no strong variation in alignment in the proposed continuum shape resonance region around $315 \mathrm{eV}$. Because the $33_{1}$ and $2 t_{2}$ molecular orbitals are inextricably convoluted in our "Auger" peak, we are inclined tentatively to conclude that this ronzero value of the observed asymmetry of this peak may be largely ascribed to these orbitals.

The results for the Cls peak are presented in Fig. 17. The Cls relative cross section was scaled in a fashion similar to the $C(K V V)$ yielc, but at $310 \mathrm{eV}$. The yield $a(\varepsilon)$ for the Cls peak has a broad shape resonance centered at $315 \mathrm{eV}$. The width of the resonance agrees with the electron impact measurements. Because of the difficulty in 
digitizing the weak shape resonance region of the carbon $k$-shell for the electon impart measurements, those data are rot plotted below 310 eV.

The Cls asymmetry parameter $\mathrm{B}(c)$ of $\mathrm{CF}_{4}$ starts at a value of -0.6 at $305 \mathrm{ev}$, goes through a broad minimum value of about 0.3 , steadily increases, and remains above 1.0 from $330 \mathrm{eV}$ to $350 \mathrm{eV}$. The width of the minimum falls between the $\mathrm{CO}$ and $\mathrm{CO}_{2} \mathrm{Cls}$ results. This width is related to the depth of the potential barrier for the cis photoelectron, and the ordering $\mathrm{CO}<\mathrm{CF}_{4}<\mathrm{CO}_{2}$ is consistent with the Cls molecular shape resonance contrast ratio having the same order. Theoretical studies of the Cls cross section $\sigma(\varepsilon)$ and asymetry parameter $B(\varepsilon)$ are needed. These experimental results appear to be useful in qualitatively describing the systematics of shape resonance phenomena.

E. OCS Results and Discussion

The ground state electronic configuration of OCS can be written : $1 \sigma^{2} 2 \sigma^{2} 3 \sigma^{2} 4 \sigma^{2} S 2 p^{6} 6 \sigma^{2} 7 \sigma^{2} 8 \sigma^{2} 9 \sigma^{2} 2 \pi^{4} 3 \pi^{4},{ }^{1} \Sigma^{+}$. We present angle resoived studies of the 30 orbital, which corresponds to the C1s shell with a binding energy of $295.2 \mathrm{eV}$, and the sulfur $\mathrm{L}_{2,3}(2 \mathrm{p})$ doublet, with edges located at 170.6 and $171.6 \mathrm{eV}$. We have not attempted to resolve the two sulfur photoelectron peaks, and will adopt an average ionization threshold of $171 \mathrm{eV}$ for this shell, which we henceforth denote as 52p. The carbon K-shell studies will be presented first, followed by the 52p and the $S(L V Y)$ results. Spectra of OCS can be found in Fig. 18 and Fig. 19. 
The $\mathrm{C}(\mathrm{KW})$ and $\mathrm{Cls}$ results are shown in Fig. 20 and $\mathrm{Fig} .21$. Our measurements will be compared to the electron energy loss measurements of Wight and Brion ${ }^{37}$ and to the MSWXa calculation on the Cls channe? given by $G r i m m^{32}$. From our earlier results, the $B(c)$ yalues calculated for the localized hole potential model was expected to show better agreement th our measurements than the unlocalized-hoie MSH calculation of Grimm, and indeed this is the case.

The $30 \rightarrow 4 \pi\left(\pi^{*}\right)$ discrete resonance have been observed by the electron energy loss measurements, 37 where the largest is centered at $288 \mathrm{eV}$. Wight and Brion have also observed discrete structure in the continuum range. ${ }^{37}$ Grimm ${ }^{32}$ has predicted that the excitations of the Cls electron into the continuum would show two shape resonances. The $\ell=3,4$ partial waves are expected to have delayed onsets. We therefore sought evidence to test this prediction.

The OCS $C(K W V)$ Auger cross section $\sigma_{A}(h v)$, shown in Fig. 20 , shows a large discrete transition below the Cls threshold. Above threshold there seems to be evidence for two maxima in the cross section, near $305 \mathrm{eV}$ and $310 \mathrm{eV}$, which couid be the $f$ and g partial wave shape resonance features predicted by Grimm. The electron impact measurements, ${ }^{37}$ which correspond to the solid curve have been scaled to our data at $291.2 \mathrm{ev}$. The electron energy loss measurements of Wight and Brion ${ }^{37}$ are expanded $(\times 3)$ to show the pre-edge discrete transitions for the Cls shell of ocs. The valence peaks intensities are apparent in the measurements below $285 \mathrm{eV}$ photon energy. The 
results of Wight and Brion ${ }^{37}$ show a much smaller intensity for energies above tha carbon $k$-edge than do the present data, probably because our measurements were performed with a 4 el monochrosator bandpass (FWHAl) compared with the 0.5 el electron energy resolution measurements of Wight and Brion. 37

The $C(K Y V)$ Auger asyminetry parameter $B_{A}\left(h_{v}\right)$ is presented in the bottom panel of Fig. 20. Again $\varepsilon_{A}(\mathrm{kv})$ is nearly zero at the discrete * resonance, as was true for all the other molecules. The value of ${ }_{A}^{B}(h v)$ generally is between -0.2 and zero through the entire energy range, although there is an increase near the Cls threshold. This also is a common feature for the $C(\mathrm{KVV})$ Auger peaks. It may be the consequence either of an alignment caused by other discrete transitions or of the valence contributions under the Auger peaks. A broad minimum is present in the asymmetry parameter near $310 \mathrm{eV}$, which may be a result of an alignment caused by a shape resonance.

The Cls cross section $o(\varepsilon)$, shown in Fig. 21, has two maxima near $305 \mathrm{eV}$ and $310 \mathrm{eV}$. Because these features were also observed for the $C$ (KVV) seak, we are inclined to believe that these two maxima are really present. If the first and second maxima are indeed the $f$ and $g$ partial wave shape resonances, respectively, then the g-wave experiences a stronger resonance.

In the bottom panel of Fig. 21 the CIs asymmetry parameter Bíc) data are shown. The localized-hole MSMXa results of Grimm are represented by the solid curve. We have lowered Grimm's curve by 0.3 units in beta. Altrough scatter is present in the data, it is clear 
that the MSMXa calculation predicts the shape of our resuits. Two minima are observed, wich would agree with the two shape resonances predicted by the MSMXa calculation.

The sulfur $2 p$ shell has been studied previously in the region of the sulfur- $L$ edges by optical absorption ${ }^{38,39}$ and electron impact methods. 37 Our work is the first photoemission investigation for the sulfur 2p shell of carbonyl sulfide near the sulfur-L edges. He shall present results for the S2p and the S(LVV) channels. The 52p shell of ocS closely resembles the S2p shell in atomic sulfur. Deviations from atomic theoretical predictions might therefore highlight specifically molecular effects in S2p photoemission. The S(LVV) results will complement and test these interpretations.

The 52p photoemission measurements were performed over the photon energy range 160-190 eV. For these measurements, the time structure of the synchrotron radiation source was poor. It had a period of 195 ns between equally spaced bunches. Each bunch contained four pulses, spaced by $\sim 2.8 \mathrm{~ns}$. As a consequence the low kinetic energy peaks were substantially broadened. Because of the high count rate in these studies, spectra were obtained after $300 \mathrm{sec}$, yielding data with excellent counting statistics. The data are presented in Figs. 22 and 23, along with the electron energy loss results. ${ }^{37}$ The solid curves in these figures are used only to connect data points. We have scaled the electron impact data to ours as previously described.

There is excellent agreement between the electron energy loss measurements and the relatively sparse data for the S(LVV) Auger 
channel, as shown in Ffg. 22. The large discrete resonances are associated with the excitation of the S2p electron to the $4 \pi\left(\pi^{*}\right)$ unoccupied molecular orbital. The $S$ (Lvv) Auger cross section $\sigma_{A}\left(h_{v}\right)$ has a maximum near $176 \mathrm{eV}$ and slowly decreases at higher energies, following the electron loss curve quite well. There is a small feature near $179 \mathrm{el}$. Wight and Brion reported observing a feature near $191 \mathrm{eV}$ wich they suggested might be caused by shake-up structure. We note these features because Allan et al. ${ }^{35}$ have shown that S2p shake-up states 1 ie 9.6 and $15.3 \mathrm{eV}$ above the sulfur $L_{2,3}$ edges in ocs.

The $\beta_{A}(h v)$ results for the $S(L W V)$ channel are also presented in Fig. 22. Table IV lists $B_{A}\left(h_{v}\right)$ and ${ }^{B_{T}}\left(h_{v}\right)$ values. The $B_{A}\left(h_{v}\right)$ parameter includes contributions from oCS valence ionic channels, which could not be resolved out. It is therefore appropriate to note the trends in $\beta_{A}\left(h_{v}\right)$, but not to draw any conclusions based solely on its precise value. The asymmetry parameter starts at a value near 1.0 below the S2p threshold and decreases sharply to near zero at 165 eV. Then $B_{A}(h v)$ has a small maximum near 167 eV (possibly due to alignment), decreases back to zero and varies little from 172 to 179 eV. The data near $179 \mathrm{eV}$ suggest there is evidence for a small degree of alignment.

The S2p results are shown in Fig. 23. In the top panel the results of Wight and Brion ${ }^{37}$ are again shown. The cross section of the S2p has a maximum at $176 \mathrm{eV}$ and slowly decreases over the rest of the energy range. The comparisons made with this work have been 
discussed in connection with the S(LVV) cross sections. Therefore those findings are identical for the $\$ 2 p$ when the excitation energy is above the sulfur $L_{2,3}$ edges.

The OCS S2p asymmetry $B(c)$ is preserited in the bottom panel of Fig. 23. The data are connected by the solid curve only to emphasize trends. The asymmetry parameter has a minimum near $179 \mathrm{eV}$, increases to about 0.1, and again drops toward zero above $185 \mathrm{eV}$. The S(LWV) Auger cross section $\sigma_{A}\left(h_{v}\right)$ showed a weak feature near $180 \mathrm{eV}$, which coincides with a threshold for a \$2p shake-up state.

The changes of the $52 \mathrm{p}$ asymmetry parameter $B(c)$ near $180 \mathrm{eV}$ and above 185 eV suggests that $B(\varepsilon)$ might be sensitive to the population of shake-up states. In Fig. 24 a spectrum taken at 190 eV clearly shows the S2p shake-up peaks. We have determined that the binding energies of these states are about 9.5 (S2) and $15.0 \mathrm{eV}$ (S1) above the sulfur $2 p$ edges with intensities that are $6.3 \neq 0.6$ percent and $11.7 \pm 0.7$ percent of the S2p peak, respectively. Using $\mathrm{Mg} \mathrm{Ka}$ radiation, Allan et al. ${ }^{30}$ found that the shake-up peaks were $4.8 \pm$ 1.5 percent and $6.4 \neq 2.4$ percent of as intense as the S2p peak. We shall treat the molecular photoionization of the $52 p$ shell in OCS in a "quasi-atomic" model, as if it were the ionization of an atomic $2 p$ subshell. We exercise caution due to limitations in this approach because of molecular dynamical effects such as shape resonances, anisotropic ion interactions arising from the asymetric molecular field, and higher angular momentum states must be included to describe fully the molecular system. In particular, we shall 
discuss the dynamics of the mfasured $s(c)$ parameter, using the atomic model presented by Manson. 40

In the Cooper-2are $(C-Z)$ formalism, ${ }^{41}$ the asymetry parameter $B(\varepsilon)$ can be written

$$
B(\varepsilon)=\frac{(\ell-1) R_{\ell-1}^{2}(\varepsilon)+(\ell+1)(\ell+2) R_{\ell+1}^{2}(\varepsilon)-\sigma_{\ell}(\ell+1) R_{\ell-1}^{2}(\varepsilon) R_{\ell+1}^{2}(\varepsilon) \cos \Delta(\varepsilon)}{(2 \ell+1)\left[\ell R_{\ell-1}^{2}(\varepsilon)+(\ell+1) R_{\ell+1}^{2}(\varepsilon)\right]}
$$

The radial dipole matrix elements are given by $R_{\ell \pm 1}$, where $\ell$ is the angular momentum of the specific orbital that is ionized and $\Delta(\varepsilon)$ accounts for the algebraic sum of the two phase differences for the interfering $\ell \pm 1$ channels:

$$
\Delta(\varepsilon)=\left[\delta_{\ell+1}(\varepsilon)-\delta_{\ell-1}(\varepsilon)\right]+\left[n_{\ell+1}(\varepsilon)-n_{\ell-1}(\varepsilon)\right]
$$

The $s_{\ell \neq 1}$ and $n_{\ell \pm 1}$ terms correspond to the non-Coulomb and Coulomb phase shifts, respectively. It is assumed that both of the $\delta_{\ell \neq 1}$ vary slowly with energy. ${ }^{40}$ The Coulomb phase shift given by Manson is expressed as

$$
n_{\ell+1}(\varepsilon)-n_{\ell-1}(\varepsilon)=\tan ^{-1}\left[-(2 \ell+1) /\left(\varepsilon^{1 / 2} \ell(\ell+1)-\varepsilon^{-1 / 2}\right)\right]
$$

For $2 p$ ionization, $p \rightarrow \varepsilon d$ and $p \rightarrow \varepsilon s$ channels are allowed by the dipole selection rules. Using Eqs. (8) and (10) and defining the ratio $o(\varepsilon)=R_{0}^{\prime}(\varepsilon) / R_{2}(\varepsilon)$, we have 


$$
B(c)=\frac{1-2 p(\varepsilon) \cos \Delta(c)}{1+.5 p(c)^{2}} \text {. }
$$

Assuming $\delta_{s}(0) \approx 2 \pi$ and $\delta_{d}(0) \approx 0$, and that both vary slowly with energy, the $\cos \Delta(\varepsilon)$ can be written approximately as

$$
\cos \Delta(\varepsilon)=\cos \left[\tan ^{-1}\left(-3 \varepsilon^{1 / 2}\right) /(2 \varepsilon-1)\right]
$$

Hence the radial matrix element ratio $p(\varepsilon)$ can be determined as the solution of the quadratic equation

$$
\rho(\varepsilon)^{2}+\rho(\varepsilon) \frac{4 \cos \Delta(\varepsilon)}{B(\varepsilon)}+2-\frac{2}{B(\varepsilon)}=0
$$

which are

$$
p(\varepsilon)=\frac{-2 \cos \Delta(\varepsilon)}{B(\varepsilon)} \pm\left[\frac{4 \cos \Delta(\varepsilon)^{2}}{B(\varepsilon)^{2}}+\frac{2}{B(\varepsilon)}-2\right]^{1 / 2}
$$

An examination of $\rho(\varepsilon)$ requires that the positive root be taken. Using this solution for $\rho(\varepsilon)$, together with our $B(\varepsilon)$ values, we have determined that the value of $o(\varepsilon)$ is about 0.5 . This confirms that the matrix element for the $p \rightarrow E S$ channel is always smaller than for the $p \rightarrow \varepsilon d$ channel, as suggested by Manson. 40

The coulomb phase shift difference and asymmetry parameter determined using the quasi-atomic model is shown in Fig. 25 . The values for radiai fatrix element branching ratio $\rho(\varepsilon)$ lie between 0.40 and 0.54 . The $p \rightarrow \varepsilon s$ channel contribution to photoionization should 
generally decrease slowly with increasing excitation energy. Therefore deviations from that behavior probably arise from explicit molecular structural effects. In fig. 25 it is evident that the radial matrix branching ratio ofe) shows abrupt increases near $179 \mathrm{eV}$ and $186 \mathrm{eV}$, energies that correspond to the edge energies of the two strong satellites.

Using the atomic non-Coulomb phase shifts provided by Manson ${ }^{42}$ the same quasi-atomic calculation was performed. It was determined that the radial matrix element ratio increased very rapidly over the photon energy range of 174-190 eV. By $187 \mathrm{eV}$ the ratio of the dipole matrix elements is larger than 10 . This suggests that the rise in the $p \rightarrow d$ non-Coulomb phase shift predicted from the atomic calculation of Manson $^{42}$ is increasing too rapidly, which may show that the potential barrier in OCS prevents the rise in the $p \rightarrow d$ non-Coulomb phase shift from occuring over the energy range of $h_{v}=174-190 \mathrm{eV}$. The Coulamb phase shift differences, asymmetry parameter, and the radial matrix element ratio calculated by the two above methods are listed in Table $V$. If the $p \rightarrow \varepsilon d$ channel is correlated with the S2p satellite states, the radial matrix branching ratio o( $\varepsilon$; could possibly increase as these new channels were opened. This would imply either that the $p \rightarrow$ ed channel had decreased or that the $p \rightarrow \varepsilon s$ channel was enhanced. Correlation effects for the $5 p^{6}$ sub-shell of Xe have been observed experimentalty. ${ }^{43,44}$ Amusia and Ivanov ${ }^{45}$ have accounted for the correlation effects on the $5 p \rightarrow c 5$ and $5 p \rightarrow e d$ channels using the random-phase approximation with exchange. It was found that the 
photoionization cross section for the $5 p$ shell was only weakly affected by correlation to the $4 \mathrm{~d}$ she11, but larger changes were observed in the angular asymmetry parameter $6(\varepsilon)$ of the $5 p .{ }^{46}$ A similar correlation for OCS in the S2p she11 and the 3x and 80 molecular orbitals could explain the peculiar variation of $p(c)$ and $B(c)$ at the S2p satellite threshold energies. A theoretical study of the effect of shake-up states on the S2p ionization channels is warranted to explain fully the observed variation in cross section $\sigma(\varepsilon)$ and angular asymmetry parameter $B(\varepsilon)$. 


\section{F. Conclusions}

Many conclusions could be drawn from our experimental studies of the carbon $\mathrm{K}$-shells of $\mathrm{CO}, \mathrm{CO}_{2}, \mathrm{CF}_{4}$, and $\mathrm{OCS}$, the oxygen $\mathrm{K}$-shells of $\mathrm{CO}$ and $\mathrm{CO}_{2}$, and the S2p shell of OCS. The following conclusions are representative rather than exhaustive.

(1.) The near-threshold electron distributions for the $\mathrm{Cls}, 01 \mathrm{~s}$, $\mathrm{C}(\mathrm{KVV})$ Auger, and $\mathrm{O}(\mathrm{KVV})$ Auger peaks of $\mathrm{CO}$ and $\mathrm{CO}_{2}$, the $\mathrm{Cls}$ and $C(K V V)$ Auger peaks of $\mathrm{CF}_{4}$ and $O C S$, and the S2p and S(LVV) Auger peaks of OCS have been determined by measuring the cross sections and asymetry parameters for these molecular systems.

(2.) Shape resonances were observed in both the cross section and asymmetry parameter for all of the Cls photoionization channels in every molecule. The Cls cross section and asymetry parameter for $\mathrm{CO}_{2}$ shows the most dramatic effects, in the $\ell=2,3$ shape resonance region. The $C 1 s$ measurements of OCS may show two shape resonances, where $\ell=3,4$ partial waves would be the successive dominant ionic channels. The Cls results of $\mathrm{CF}_{4}$ are described in the scope of shape resonance phenomena, although no theoretical predictions are available, because of similarities to the other molecules in the variation of the cross section and the asymmetry parameter. 
(3.) The $C(K V V)$ partial cross sections show both the discrete resonances below the Cls ionization thresholds and the shape resonances in the cont inuum. The Auger asymmetry parameter shows no strong alignment at the $\pi$ discrete resonances of $\mathrm{CO}$, $\mathrm{CO}_{2}$, and $\mathrm{OCS}$ and the $3 \mathrm{Sa}_{1}$ resonance of $\mathrm{CF}_{4}$. Two alternative explanations were offered, of which a "spectator" excited electron is the more appealing. Higher-resolution experiments are clearly needed. All the C(KVV) Auger peaks show small net alignments between the largest discrete resonance and the Cls ionization threshold which may be caused by other discrete resonances, al though interference from valence shells is a problem. The shape resonances have small effects, or no effect, in the continuum region on the asymmetery parameter of the $C(K V V)$ in all of the molecules. Small, but nonzero, asymmetries are observed. Aga in valence-shell interference cannot be ruled out.

(4.) The partial cross sections for the $O(K V V)$ and $01 \mathrm{~s}$ peaks of $C O$ and $\mathrm{CO}_{2}$ clearly show the $01 \mathrm{~s}$ shape resonance. The asymmetry parameter of the $O$ (KVV) of $C O$ shows an alignment in the shape resonance region.

(5.) The Cls photoionization cross section of $\mathrm{CO}_{2}$ given by the electron-ion coincidence measurements of Kay et al. ${ }^{11}$ is in good agreement with our results. 
(6.) The electron energy loss results of Wight et al. ${ }^{6}$ and wight and $B$ rion $8,9,37$ in the vicinity of the carbon K-edge, oxygen K-edge, and sulfur $L_{2,3}(2 p)$ edges, for the cross sections are in good agreement with the present measurements.

(7.) The photoabsorption measurements of Barrus et al. ${ }^{3}$ for the oxygen is shell of $\mathrm{CO}$ and $\mathrm{CO}_{2}$ are in excellent agreement with the present results.

(8.) The STMT partial cross sections of Padial et al. ${ }^{14}$ for the CIs and 0ls channels of $C O$ are in excellent agreement with the results. For $\mathrm{CO}_{2}$ the $\mathrm{Cls}$ shape resonance is located too close to threshold, 15 and the $01 \mathrm{~s}$ cross section shape is only in qualitative agreement with the present results.

(9.) The partial cross sections and asymmetry parameters $B(\varepsilon)$ predicted from the MSMXa calculations are in qualitative agreement with the experimental results. ${ }^{30-32}$ This model works well in identifying possible shape resonance features and in some cases predicts the location of the shape resonances in good agreement with the experimental measurements, but some discrepancies are noticed in particular between the calculated $01 \mathrm{~s}$ asymmetries in $\mathrm{CO}$ and $\mathrm{CO}_{2}$ and the present results. The MSMXa results of Grimm ${ }^{32}$ using the localized-hole potential model is generally in better 
agreement with the results than is the unlocalized hole potential model.

(10.) The HF static exchange calculations by Lucchese and Mckoy 16 for $\mathrm{CO}_{2}$ show promise in correctly describing the $\mathrm{Cls}$ asymmetry parameter. The Cls photoionization cross section is predicted with the correct shape, but the maximum is located too close to threshold.

(11.) Our measured intensity ratio of an $01 \mathrm{~s}$ shake-up feature to the $01 \mathrm{~s}$ main line of $\mathrm{CO}$ at a photon energy of $630 \mathrm{eV}$ is very nearly the same as that measured with $\mathrm{Mg} \mathrm{Ka}$ radiation by $\mathrm{All}$ an et. al. 35 The unrestricted Hartee-Fock calculation by Aarons et al. ${ }^{34}$ predicted the ratio of the total shake-up peak intensity to the 015 main-line photoelectron peak to be about $20 \%$, in reasonably good agreement with experiment.

(12.) The asymmetry parameter for the S2p channel of OCS may be sensitive to the production of S2p satellite peaks. We observed a peculiar variation in the asymmetry parameter at photon energy corresponding to threshold energies of satellite states.

(13.) A quasi-atomic calculation for the $52 p$ channel in oCS, using the Cooper-Zare model, was useful in determing the ratio of 
the two radial matrix element: $\partial(c)$ from the asyminetry parameter and the phase shift differences for the es and $\mathrm{cd}$ ionization channeis. The unusual behavior of-p(c) may be related to correlation effects between the S2p and the 3r and 80 molecular orbitals, by analogy to similar phenomena in atoms. 


\section{References}

1. C.N. Yang, Phys. Rev. 74, 764 (1948).

2. D. Dill, J.R. Swanson, S. Hallace, and J.L. Dehmer, Phys. Rev. Lett. 45,1393 (1980).

3. D.M. Barrus, R.L. Blake, A.J. Burek, K.C. Chambers, and A.L. Pregenzer, Phys. Rev. A 20, 1045 (1979).

4. R.Z. Bachrach, F.C. Brown, A. Bianconi, and H. Petersen, Stanford Synchrotron Radiation Report No. 77/16.

5. M. Tronc, G.C. King, R.C. Bradford, and F.H. Reed, J. Phys. B: Atom. Molec. Phys. 9, L555 (1976).

6. G.R. Wight, C.E. Brion, and M.J. van der Wiel, J. Electron Spectrosc. Relat. Phenom. 1, 457 (1972/73).

7. M.J. van der Wiel, Th. M. El-Sherbini, and C.E. Brion, Chem. Phys. Lett. I, 161 (1970).

8. G.R. Wight and C.E. Brion, J. Electron Spectrosc. Relat. Phenom. $\underline{3}$, 191 (1974).

9. G.R. Wight and C.E. Brion, J. Electron Spectrosc. Relat. Phenom. 4, $327(1974)$.

10. A.P. Hitchcock and C.E. Brion, J. Electron Spectrosc. Relat. Phenom. 18, 1 (1980).

11. R.B. Kay, Ph. E. van der Leeuw, and M.J. van der Wiel, J. Phys. B: Atom. Molec. Phys. 10, 2513 (1977). 
12. J. Stöhr, K. Baberschke, R. Jaeger, R. Treichler, and S. Brennan, Phys. Rev. Lett. 47, 381 (1981).

13. C.L. Allyn, T. Gustaffson, and E.H. Plumer, Chen. Phys. Lett. 4, 127 (1977); C.L. Allyn, T. Gustaffson, and E.H. Plummer, Solid State Commun. 24, 531 (1977).

14. N. Padial, G. Csanak, B.V. McKoy, and P.W. Langhoff, J. Chem. Phys. 69, 2982 (1978).

15. N. Padial, G. Csanak, B.V. McKoy, and P.W. Langhoff, Phys. Rev. A 23, 218 (1981).

16. R.R. Lucchese and B.V. Mckoy, Phys. Rev. A 26, 1406 (1982).

17. M.G. White, R.A. Rosenberg, G. Gabor, E.D. Poliakoff, G. Thornton, S. Southworth, and D.A. Shirley, Rev. Sci. Instrum. 50, 1288 (1979).

18. S. Southworth, C.M. Truesdale, P.H. Kobrin, D.W. Lindle, W.D. Brewer, and D.A. Shirley, J. Chem. Phys. 76, 143 (1982).

19. F. Wuilleumier and M.O. Krause, J. Electron Spectrosc. Relat. Phenom. 15, 15 (1979).

20. F.C. Brown, R.Z. Bachrach, and N. Lien, Nucl. Instrum and Meth. 152,73 (1979).

21. C.M. Truesdale, S. Southworth, P.H. Kobrin, U. Becker, H.G. Kerkhoff, and D.A. Shirley, submitted to Phys. Rev. Lett.

22. K. Siegbahn, C. Nordling, G. Johannson, J. Hedman, P.F. Heden, K. Mamrin, U. Gelius, T. Bergmark, L.O. Werme, R. Manne, and V. Baer, ESCA Applied to Free Molecules, North-Holland Publ. Co., Ams terdam, 1969. 
23. H.E. Moddeman, T.A. Carlson, M.O. Krause, B.P. Pullen, W.E. Bull, and G.K. Schweitzer, J. Chem. Phys. 55, 2317 (1971).

24. J.A. Kelber, D.R. Jennison, and R.R. Rye, J. Chem. Phys. 75, 652 (198I).

25. H. Agren, J. Chem. Phys. 75,1267 (1981).

26. Private communication with $T$. Darrah Thomas, Oregon State University, Corvalis, Oregon 97331.

27. L. Ungier, J.K. Gimzewski, and T.D. Thomas, Proc. X-B2 Conf. on Atomic Inner Shell Phys. Eugene, Abstracts, 245 (1982).

28. J.L. Dehmer and D. Dill, Phys. Rev. Lett., 35, 213 (1975).

29. J.L. Dehmer and D. Dill, J. Chem. Phys. 65, 5327 (1976).

30. D. Dill, S. Wallace, 1. Siegel, and J.L. Dehmer, Phy:.. Rev. Lett. 42, 4.11 (1979).

31. J.L. Dehmer and D. Di11, Argonne National Laboratory Report (ANL-77-65) p. 65.

32. F.A. Grimm, Chem. Phys. 53, 71 (1980).

33. T.A. Carlson, M.0. Krause, and W.E. Moddeman, J. de Physique 32, C4-76 (1971).

34. L.J. Aarons, M. Barber, M.F. Guest, I.H. Hillier, and J.H. Me Cartney, Mol. Phys. 26, 1247 (1973).

35. C.J. Allan, U. Gelius, D.A. Allison, G. Johansson, H. Siegbahn, and $X$. Siegbahn, J. Electron Spectrosc. Relat. Phenom. 1, 131 (1972/73).

36. C.R. Brundle, M.B. Robin, and H. 8asch, J. Chem. Phys. $\underline{53}, 2196$ (1970). 
37. G.R. Wight and C.E. Brion, J. Electron Spectrosc. Relat. Phenom. 4, 327 (1974).

38. L.M. Mazlov, A. P. Sadovskij, V.M. Bertenev, K.E. Mironov, T.I. Guzhavina, and L.1. Chernyavskii, 2h. Struct. Chem. 13, 859 (1972); (J. Struct. Chem. 13, 802 (1972))

39. A.S. Vinogradov and T.M. Zimkina, Opt. Spectrosk. 31, 685 (1971) (Opt. Spectrosc. (U.S.S.R.) 31, 364 (1971)).

40. S.T. Manson, J. Electron Spectrosc. Relat. Phenom. 1, 413 (1972/73).

41. J. Cooper and R.N. Zare, Lectures in Theoretical Physics, edited by S. Geltman, K.T. Mahanthappa, and W.E. Britten (Gordon and Breach, New York) Vol XI. - C pp. 317-337 (1969).

42. S.T. Manson, Phys. Rev. 182, 97 (1969).

43. G.V. Marr, in Photoelecton Emission: Proceedings of the Daresbury Study Weekend, 6-7 March 1976, DL/SRF/128 p. 81.

44. 5. Southworth, U. Becker, C.M. Truesdale, P. Kobrin, D.W. Lindle, and D.A. Shirley, to be published in Phys. Rev. A.

45. M. Ya. Amusia and N.A. Cherepkov, Case Studies in Atomic Physics 5, 47 (1975).

46. M. Ya. Amusia and N.A. Cherepkov, Phys. Lett, 59A, 194 (1976). 
Table I. CO C(KVV) Auger asymmetry parameter.

\begin{tabular}{|c|c|c|}
\hline$\underline{h v}\left(\mathrm{eV}^{V}\right)$ & $B_{A}\left(h_{v}\right)$ & $B_{T}\left(h_{v}\right)$ \\
\hline 275.8 & $\longrightarrow$ & $1.08(9)^{\mathrm{a}}$ \\
\hline 280.8 & - & $1.28(6)$ \\
\hline 284.8 & $\longrightarrow$ & $1.07(6)$ \\
\hline 285.8 & $0.17(2)$ & $0.24(2)$ \\
\hline 286.8 & $0.13(8)$ & $0.46(3)$ \\
\hline 287.1 & $0.03(3)$ & $0.30(3)$ \\
\hline 287.3 & $0.08(3)$ & $0.10(1)$ \\
\hline 289.0 & $0.32(3)$ & $0.42(4)$ \\
\hline 293.0 & $0.12(5)$ & $0.25(5)$ \\
\hline 294.9 & $0.30(5)$ & $0.45(5)$ \\
\hline 296.0 & $0.37(5)$ & $0.53(5)$ \\
\hline 296.7 & $0.24(3)$ & $0.59(4)$ \\
\hline 296.8 & $0.37(4)$ & $0.54(6)$ \\
\hline 298.0 & $0.09(4)$ & $0.24(4)$ \\
\hline 300.0 & $0.08(3)$ & $0.42(3)$ \\
\hline 300.9 & $0.12(3)$ & $0.35(3)$ \\
\hline 301.8 & $0.13(3)$ & $0.30(3)$ \\
\hline 302.0 & $0.18(3)$ & $0.37(3)$ \\
\hline 302.9 & $0.10(3)$ & $0.24(3)$ \\
\hline 303.8 & $0.14(2)$ & $0.35(2)$ \\
\hline 305.0 & $0.10(3)$ & $0.38(3)$ \\
\hline 305.8 & $0.10(2)$ & $0.28(3)$ \\
\hline 307.0 & $0.16(2)$ & $0.28(3)$ \\
\hline 309.0 & $0.00(2)$ & $0.27(2)$ \\
\hline 310.0 & $0.06(2)$ & $0.31(3)$ \\
\hline 311.0 & $0.11(3)$ & $0.30(3)$ \\
\hline 313.0 & $0.08(3)$ & $0.31(3)$ \\
\hline 315.0 & $-0.09(2)$ & $0.32(3)$ \\
\hline
\end{tabular}

a Errors in the last digit are given parenthetically. 
Table II. $\mathrm{CO}_{2} \mathrm{C}(\mathrm{KVV})$ Auger asymmetry parameter.

\begin{tabular}{|c|c|c|}
\hline$h v(e V)$ & $B A(h v)$ & $B_{T}(h v)$ \\
\hline $\begin{array}{l}270.0 \\
273.0 \\
279.7 \\
282.7 \\
284.7 \\
286.7 \\
287.7 \\
289.2 \\
290.0 \\
291.7 \\
295.7 \\
299.7 \\
301.7 \\
302.7 \\
303.7 \\
305.7 \\
306.7 \\
308.7 \\
309.7 \\
311.7 \\
313.2 \\
314.7 \\
316.7 \\
318.7 \\
320.7 \\
322.7 \\
324.7 \\
330.7 \\
335.7 \\
340.7\end{array}$ & $\begin{array}{l}\bar{Z} \\
\bar{Z} \\
\bar{Z} \\
0.27(2) \\
0.21(2) \\
0.35(3) \\
0.77(4) \\
0.50(4) \\
0.20(2) \\
0.17(2) \\
0.16(1) \\
0.25(1) \\
0.15(1) \\
0.05(1) \\
0.12(1) \\
0.12(1) \\
0.10(1) \\
0.17(2) \\
0.15(1) \\
0.15(1) \\
0.07(1) \\
0.07(1) \\
0.05(1) \\
0.13(1) \\
0.36(4) \\
0.37(3)\end{array}$ & $\begin{array}{l}1.40(1) \\
1.53(1) \\
1.28(1) \\
1.33(1) \\
1.42(1) \\
1.42(1) \\
0.99(1) \\
= \\
0.45(3) \\
0.84(4) \\
0.68(4) \\
0.58(3) \\
0.55(5) \\
0.48(3) \\
0.56(3) \\
0.45(3) \\
0.30(3) \\
0.37(2) \\
0.27(2) \\
0.31(2) \\
0.39(3) \\
0.35(3) \\
0.36(3) \\
0.42(3) \\
0.38(3) \\
0.33(3) \\
0.41(4) \\
0.30(4) \\
-1\end{array}$ \\
\hline
\end{tabular}


Table 1II. Total 01s shake-up intensity relative to the 01s min line of $\mathrm{CO}_{2}$.

\begin{tabular}{ll}
\hline hv(eV) & br anching ratio(percent) \\
\hline 593.0 & $17.1 \pm 1.9$ \\
609.0 & $20.4 \neq 2.6$ \\
615.0 & $23.9 \neq 3.1$ \\
625.0 & $21.1 \neq 2.4$ \\
631.0 & $21.9 \neq 2.6$ \\
\hline
\end{tabular}

(The average value for these results is $20.8 \neq 2.7$ percent). 
165

Table IV. OCS S(LVV) Auger as ymetry parameter iA.

\begin{tabular}{lll}
\hline \hline hv(eV) & ${ }_{A}\left(h_{v}\right)$ & ${ }_{T}\left(h_{v}\right)$ \\
\hline 160.0 & $1.13(7)$ & $1.00(7)$ \\
164.0 & $0.41(4)$ & $0.85(5)$ \\
165.5 & $0.14(3)$ & $0.58(4)$ \\
166.5 & $0.08(3)$ & $0.61(4)$ \\
167.0 & $0.18(3)$ & $0.66(4)$ \\
168.0 & $0.15(3)$ & $0.67(4)$ \\
170.0 & $0.06(3)$ & $0.62(4)$ \\
172.0 & $0.01(2)$ & $0.43(3)$ \\
174.0 & $0.00(2)$ & $0.37(2)$ \\
176.0 & $-0.05(1)$ & $0.30(2)$ \\
177.0 & $-0.06(1)$ & $0.31(2)$ \\
178.0 & $-0.03(2)$ & $0.33(2)$ \\
178.5 & $-0.05(2)$ & $0.33(2)$ \\
179.0 & $-0.07(2)$ & $0.33(2)$ \\
179.5 & $-0.02(2)$ & $0.37(2)$ \\
180.0 & $-0.13(1)$ & $0.30(2)$ \\
182.0 & $-0.08(2)$ & $0.38(2)$ \\
185.0 & $-0.19(2)$ & $0.35(3)$ \\
187.0 & $-0.23(2)$ & $0.36(3)$ \\
190.0 & $-0.16(1)$ & $0.38(2)$ \\
\hline \hline
\end{tabular}


Tabie $Y$. The Coulomb phase shift difference $\Delta(c)$, where the non-Coutomb phase difference $(-2 \Omega)$ has been suppressed, the $\$ 2 p$ asymetry parameter $s(c)$ of OCS, and the ratio of the two radial matrix elements $\rho(c)$ calculated with constant non-Coulomb phase shifts $(\star)$ and atomic non-Coulomb phase shifts calculated by Manson (*t). 42

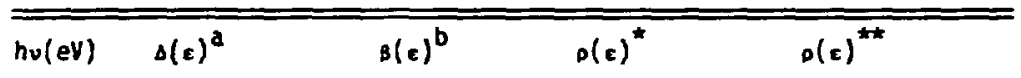

$\begin{array}{lllll}174.0 & -0.26 & 0.27(4) & 0.51(3) & 0.36(2) \\ 175.0 & -0.23 & 0.27(3) & 0.46(2) & 0.37(2) \\ 176.0 & -0.20 & 0.25(3) & 0.45(2) & 0.40(2) \\ 177.0 & -0.19 & 0.29(3) & 0.41(2) & 0.41(2) \\ 178.0 & -0.18 & 0.13(3) & 0.50(2) & 0.60(2) \\ 178.5 & -0.17 & 0.14(3) & 0.49(2) & 0.62(3) \\ 179.0 & -0.16 & 0.07(3) & 0.53(2) & 0.75(3) \\ 179.5 & -0.16 & 0.05(3) & 0.54(2) & 0.85(3) \\ 180.0 & -0.15 & 0.12(3) & 0.49(2) & 0.86(4) \\ 182.0 & -0.14 & 0.11(3) & 0.48(2) & 1.34(11) \\ 185.0 & -0.13 & 0.13(3) & 0.46(2) & 2.42(11) \\ 187.0 & -0.12 & 0.01(3) & 0.53(2) & 11.23(42) \\ 190.0 & -0.10 & 0.03(3) & 0.51(2) & 13.27(52)\end{array}$

a The Coulomb phase shift is given in units of $\pi$.

b The errors are given to the last digit parenthetically. 
Figure Captions

Figure 1. Electron tine-of-flight spectrum from $\mathrm{CO}$, after conversion to a kinetic energy scale. The sample was irradiated with photons of hv $=305.8 \mathrm{eV}$ energy (first-order), with some 2nd and 3rd-order radiation also present. Auger peaks are labelled B-1, etc., following the notation of Moddeman et al. For this spectrum the electrons were retarded by 150 volts over 60 percent of their $28 \mathrm{~cm}$ flight path.

Figure 2. Electron time-of-flight spectrum similar to Fig. 1, but with $h_{v}=315 \mathrm{eV}$ and a retarding voltage of only 5 volts, allowing the $\mathrm{Cls}$ and $\mathrm{Ols}$ (2nd-order) peaks to be recorded.

Figure 3. The $C(K V V)$ Auger results. Top panel: open circles show the total Auger intensity scaled to the results of Tronc et a.$^{5}$ The $n$ resonance at $h v=287.3 \mathrm{eV}$ is striking. Filled circles show the region above $h_{v}=290 \mathrm{ev}$, times 16 . The dashed curve represents the electron-ion coincidence measurements of Kay et al., 11 times 16 . The $\sigma$ resonance, peaking at $306 \mathrm{eV}$, is evident, as is precursor structure below the $295.9 \mathrm{eV}$ threshold. Bottom panel: filled circles show our asymmetry results. Curve shows the orientation parameter $B_{\mathrm{rn}}$ calculated by $0 \mathrm{ill}$ et al. ${ }^{2}$

Figure 4. The $C(K W V)$ Auger spectra, taken at the $\pi$ resonance (hu $=$ $287.3 \mathrm{eV}$ ) with a retarding potential of 100 volts, and at $h_{v}=296.8 \mathrm{eV}$, above the Cls threshold, with a 150 volt retarding potential. 
Figure 5. The Cls photoelectron results. Top panel: our o(c) values are represented by filled circles, while open circles show the elec. mon-ion coincidence results of Kay et al. ${ }^{11}$ The solid curve shows the STMT calculation of Padial et al., 14 and the dashed curve the MSMXa calculation by Dehmer and Di11. 31 Bottom panel: filled circles show our asymetry results. The solid and dashed curves are results from localized-hole MSMXa calculations by $0 i 11$ et al. 30 and Grimm, ${ }^{32}$ respectively.

Figure 6. The $0(K V V)$ results for $C 0$. Top panel: the experimental cross section (points) and the absorption results of Barrus et al. ${ }^{3}$ (solid curve). Bottom panel: the experimental asymmetry parameter (points) and the $B_{m}$ curve given by Dill et al. ${ }^{2}$

Figure 7. The 0ls resuits for CO. Top panel: cross section results. Filled circles are our data, open circles represent the absorption results of Barrus et al., ${ }^{3}$ and the solid curve represents the STMT calculation of Padial et al. ${ }^{14}$ Bottom panel: asymmetry data (points), compared with localized-hole MSMXa calculations by $\mathrm{Grimm}^{32}$ (solid curve) and by Dill et a). ${ }^{30}$ (dashed curve).

Figure 8. TOF spectrum of CO expanded around the 01s peak. Shake-up structure is evident. The peak labelled "S" is 13 percent as intense as the main peak. 
Figure 9. Time-of-flight spectrum of $\mathrm{CO}_{2}$, after conversion to a linear energy scale. A retarding voltage of $5 \checkmark$ was used in collecting this spectrum, but kinetic energies shown were corrected.

Figure 10. The $C(K V V)$ Auger results for $\mathrm{CO}_{2}$. Top panel: our results (filled circles) and electron energy loss results of wight and Brion, ${ }^{8}$ to which our data were norma'izf $\mathrm{J}$ at $315 \mathrm{eV}$. Bottom pane l: asymetry values. Note that some of the asymmetry points fall at energies for which there is very little spectral intensity (cf top panel) and are therefore very questionable.

Figure 11. The $\mathrm{Cls}$ results for $\mathrm{CO}_{2}$. Top panel: the cross section. Filled circles are present results, npen circles the electron energy loss results of $W i, t$ and Brion. 8 The dashed curve is the STMT predicticn by Padial et al. 15 and the solid curve represents the HF static exchange prediction by Lucchese and Mckoy. ${ }^{16}$ Bottom panel: the as ymmetry parameter. Filled ircles are present results, the solid curve is the HF calculation of Lucchese and McKoy, ${ }^{16}$ the dotted curve is the localized-hole MSM calculation by Grimm, ${ }^{32}$ and the dashed curve is the unlocalized-hole MSMXa calculation of Grimm et al. 32

Figure 12. Results for the $\mathrm{O}(\mathrm{KV} V)$ peak in $\mathrm{CO}_{2}$. Top panel: cross sections. Filled circles are present results; open circles are photoabsorption results of Barrus et al. ${ }^{3}$ The dashed 
curve and full curve display the theoretical predictions by Padial et al. 15 and by Lucchese and McKay, 16 respectively. Bottom panel: the asymmetry parameter. filled circles are the present results, connected by a line to guide the eye.

Figure 13. The asymmetry parameter for the 01s paak in $\mathrm{CO}_{2}$. The present results are shown as points. The solid and dashed curves represent predictions from localized-hole MSMXa caliulations by Grimm ${ }^{32}$ and $0 i 11$, et al., 30 respectively.

Figure 14. The 01s peaks of $\mathrm{CO}_{2}$ on an expanded scale, to show shake-up structure.

Figure 15. The TOF electron spectrum from $\mathrm{CF}_{4}$ excited at a nominal photon energy of $318.8 \mathrm{eV}$. The peaks are (left to right): Cls, C(KVV) plus inner valence states, $4 a_{1}+3 t_{2}$, le + $4 t_{2}=1 t_{1}, C 1 s$ (secund-order), and $F(K V)$ (third-order).

Figure 16. The $\mathrm{CF}_{4} \mathrm{C}(\mathrm{KVV})$ results, shown as filled circles. Top panel: the cross section curve of Bachrach et al.. 14 corrected for valence-electron contributions to the photoabsorption cross section, is shown as a curve. Jur data were normalized to this curve at $302 \mathrm{eV}$. Bottom panel: experimental asymmetry. The line through the points is shown to guide the eye.

Figure 17. The $\mathrm{Cls}$ photoelectron results for $\mathrm{CF}_{4^{*}}$. Our data are shown as filled circles. Open circles in the top panel 
show some of the electron energy loss results of wight and Brion, ${ }^{9}$ to which our data are normalized at $328 \mathrm{eV}$, ary the solid curve represents photoabsorption results $a y$ Bachrach et al. 4 The curve in the lower panel is ctrawn through the data.

Figure 18. TOF electron spectrum of OCS excjted at a photon energy of $311.0 \mathrm{eV}$. The paaks are (left to right): Cls, $52 \mathrm{~s}$ $\left(6 \mathrm{c}^{-1}\right)$, S2p (with the left shoulder being the S(LVV) Auger peak : C(KV) Auger plus valence states, and $0(K V Y)$ Auger.

Figure 19. TOF electron spectrum of OCS excited at a photon energy of $179.0 \mathrm{eV}$. Four electron pulses separated by 2.8 ns were present, which caused the S(LWV) Auger to look like four structures and the S2p to be broadened (in time).

Figure 20. Cross section and asymmetry parameter for the $C(K V V)$ of OCS. A small portion of valence peak intensity is included in the results. The solid curve in the top panel represents the electron energy loss (and $\times 3$ ) results of Wight and Brion 37 and the present measurements correspond to the filled circles. The measured asymmetry parameter is shown in the bottom pane?.

Figure 21. Cross section and asymmetry parameter for the $C(1 s)$ of ocS. The relative cross section is shown in the top panel. The measured asymmetry parameter, shown by the filled circles, and the localized-hole MSMXa calculation of Grimm is illustrated in the bottom panel. 
Figure 22. The S(LVV) results from ocS. Our cata are represented by filled circles in both panels. Open circles in the top pane 1 are electron energy loss yield results of wight and Brion, ${ }^{37}$ scaled to our data. Solid curves are drawn to connect the paints.

Figure 23. The S2p results from OCS. The notation is the same as in Figure 22.

Figure 24. An expancied TOF spectrum around the S2p peak region showing the two satellite peaks. Note that the satellites are more intense relative to the main peak than they appear, because of their greater data density.

Figure 25. Phase-shift differences and radial matrix element ratio for $52 p$ in OCS, calculated from the measured as ymmetry parameter according to a "quasi-atomic" model described in the text. The non-Coulomb relative phase differerce equal to $-2 \pi$ has been suppressed to show variation of the Coulomb relative phase shift differences for the $e s$ and $e d$ channels. 


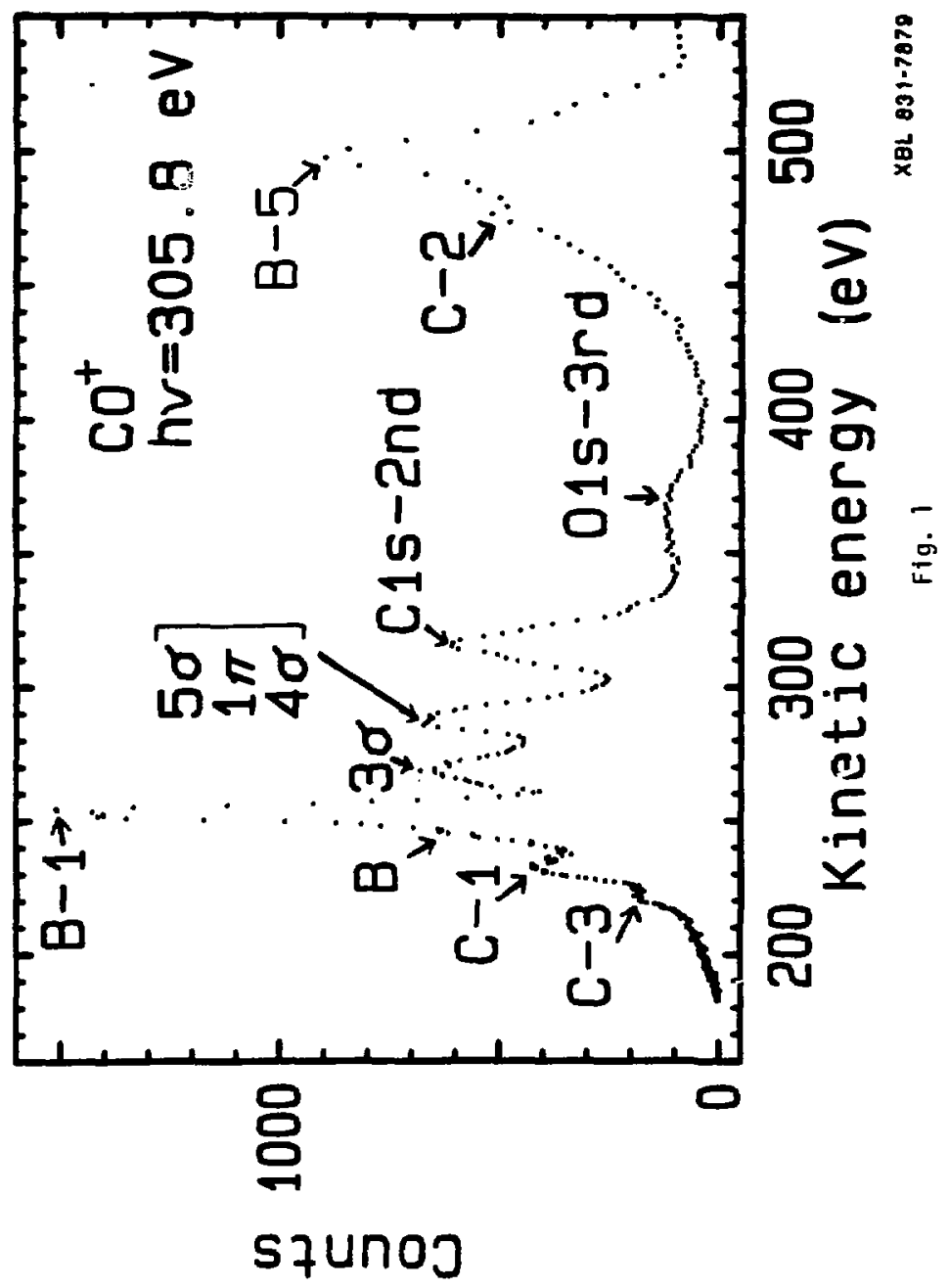




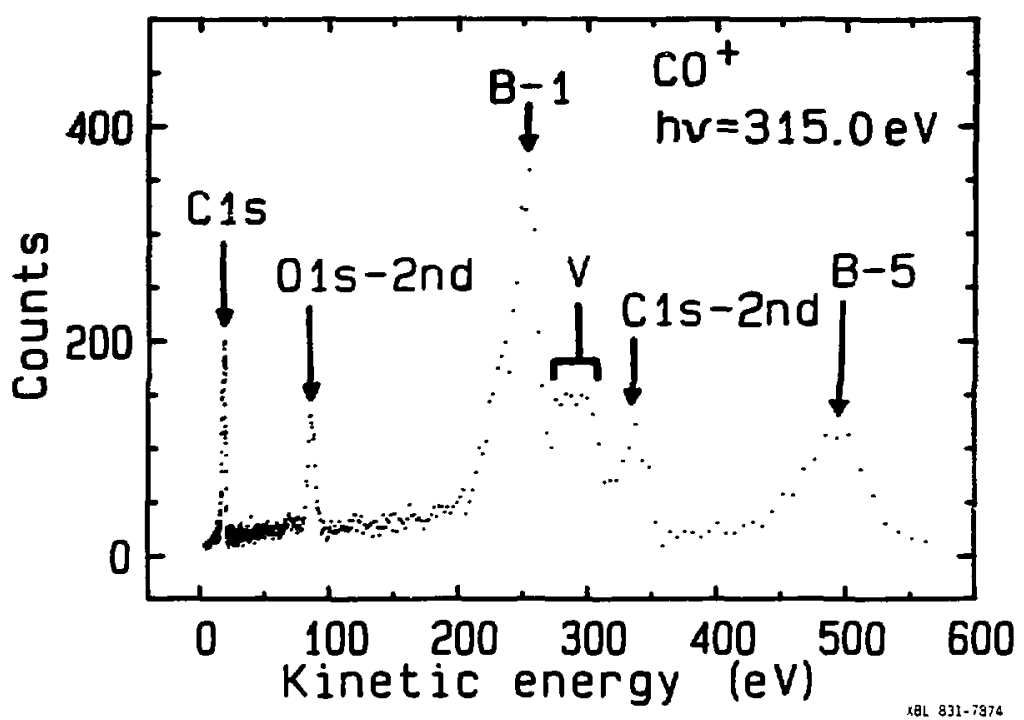

Fig. 2 


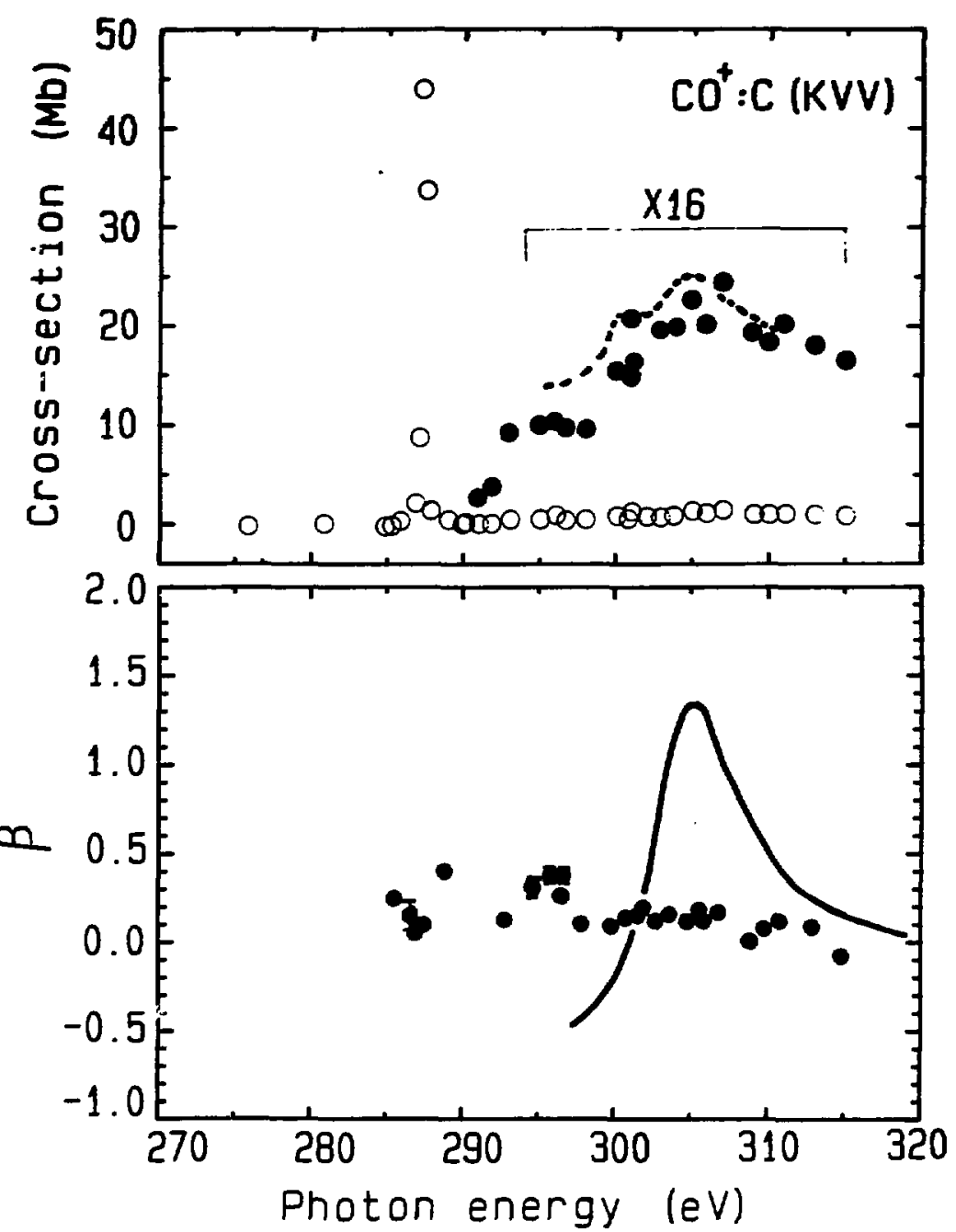

Fig. 3 


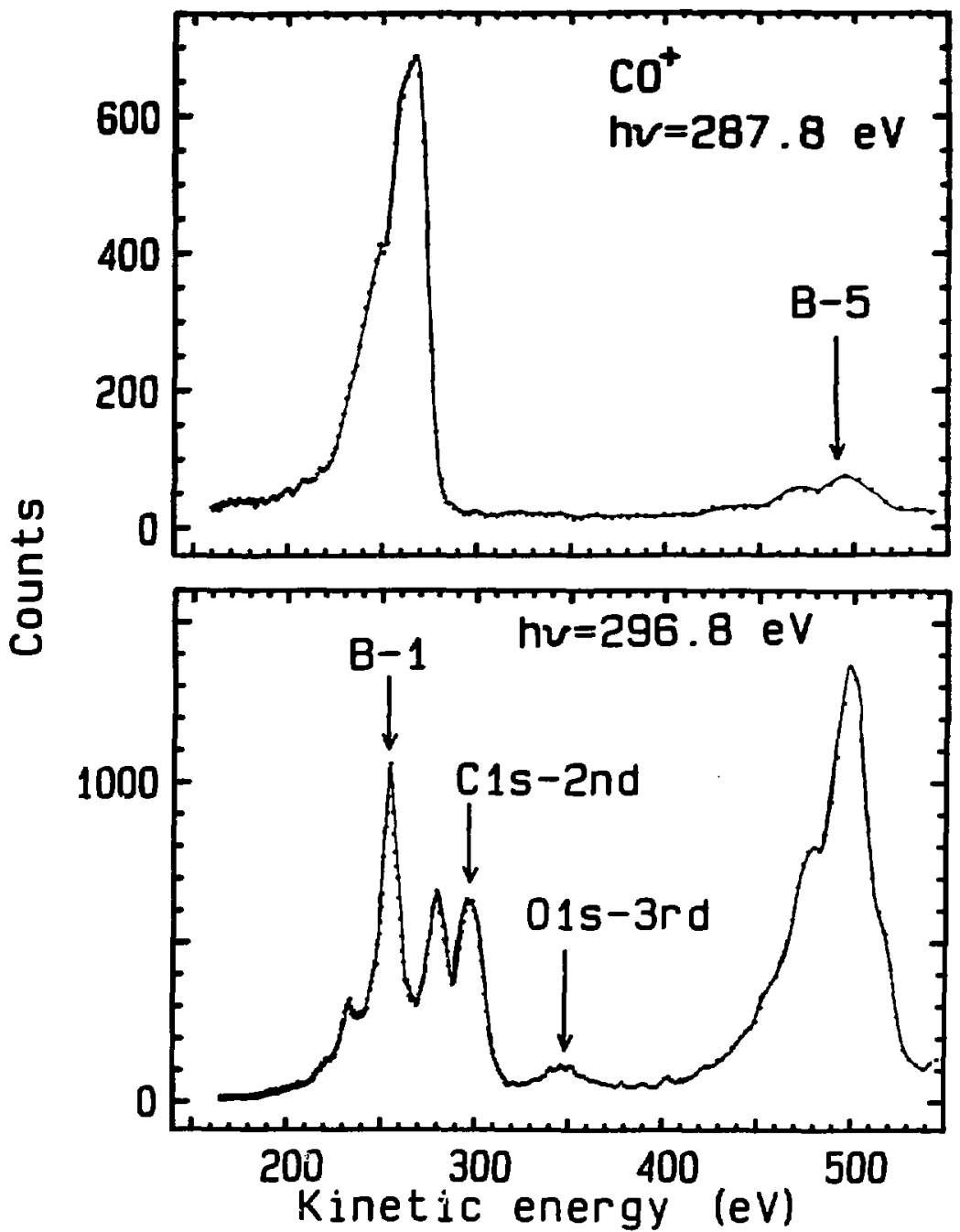

Fig. 4 


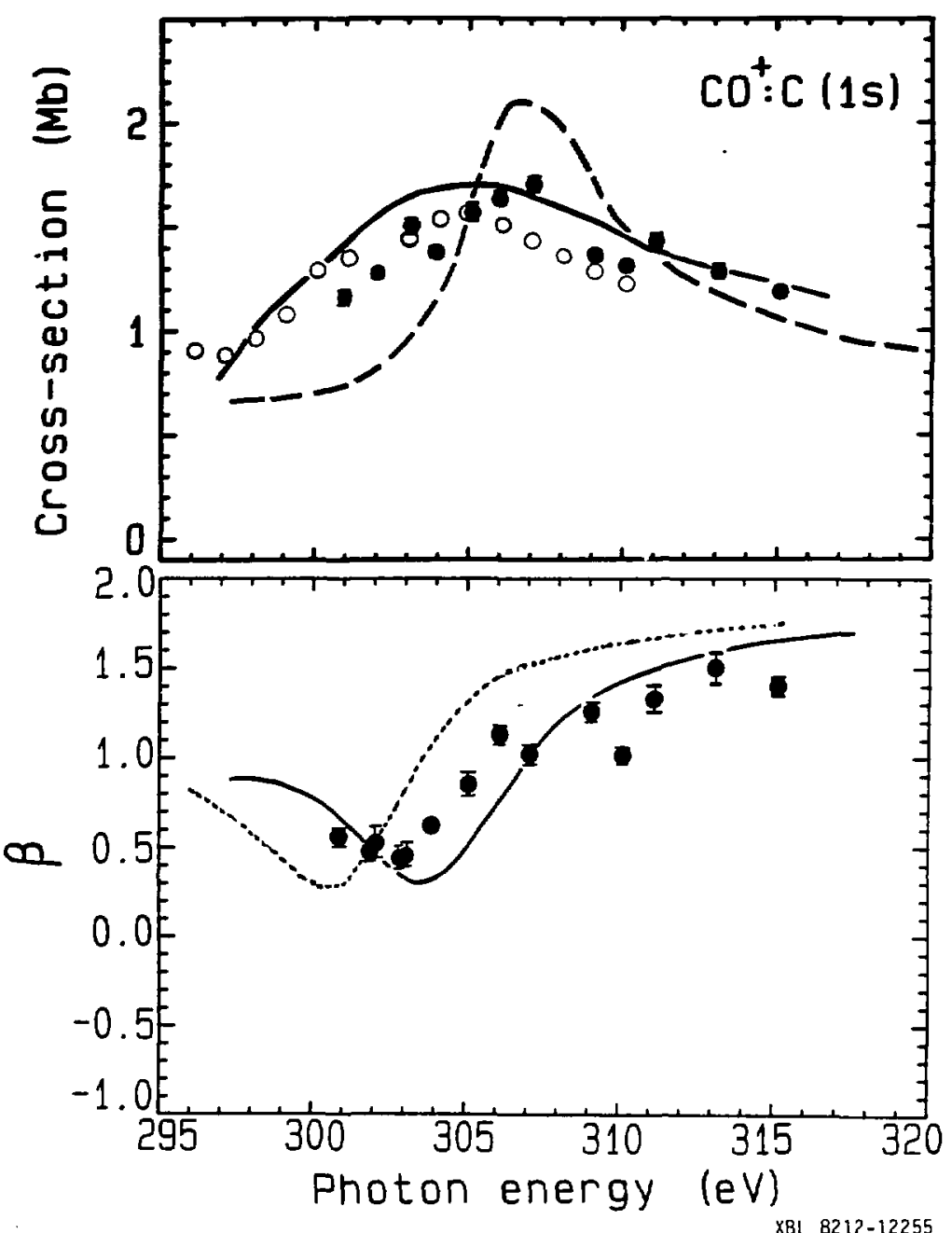

Fig. 5 

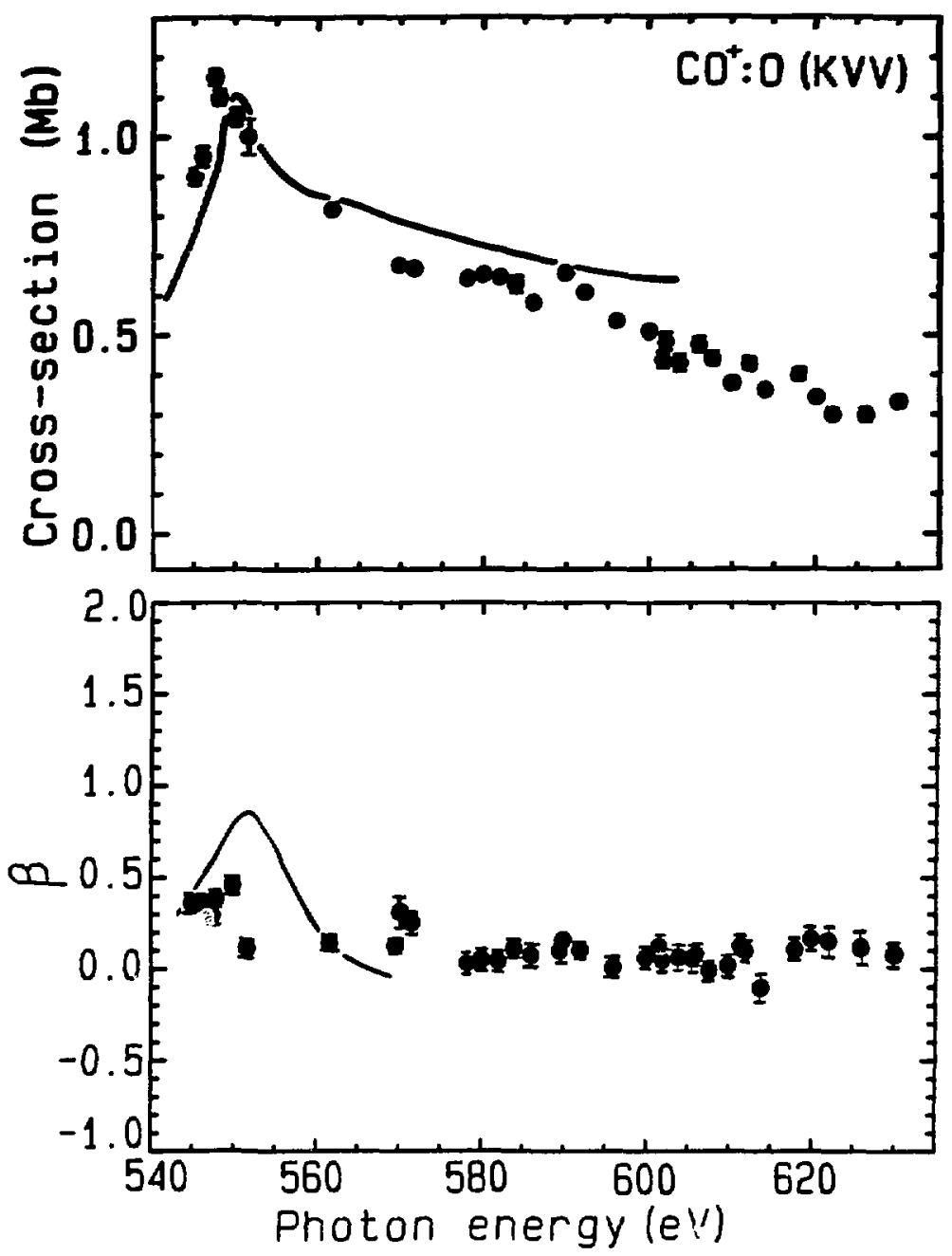

Fig. 5

XBL 828- 11017 

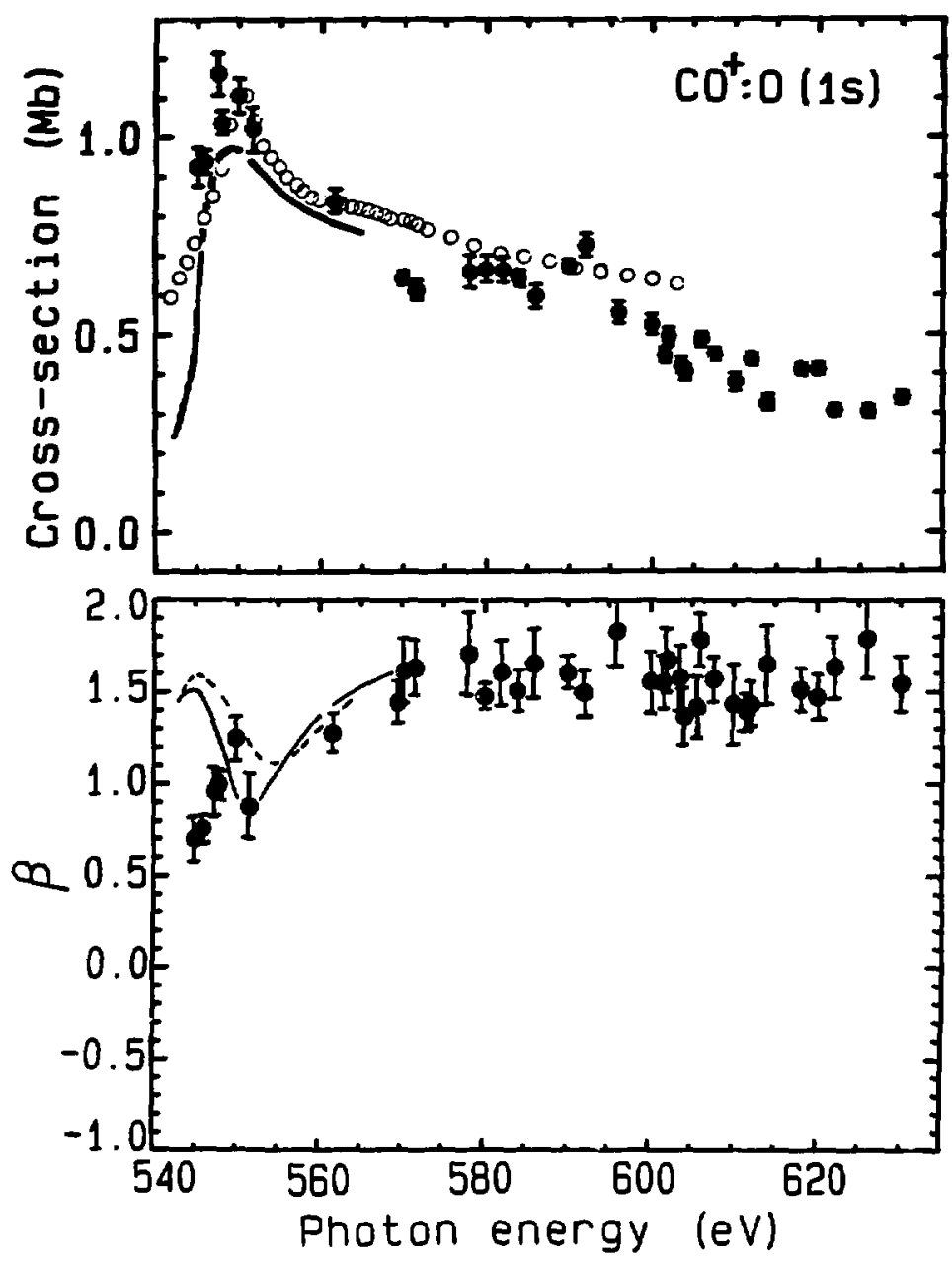

Fig. 7 


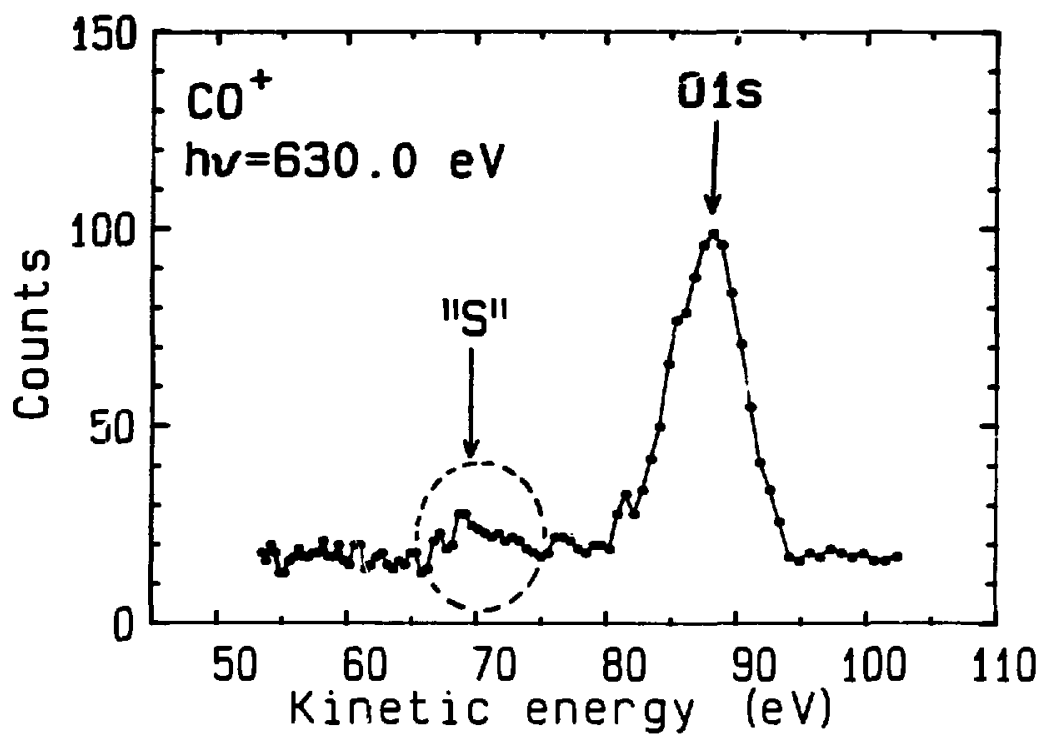

XBL 832-8333

Fig. 8 


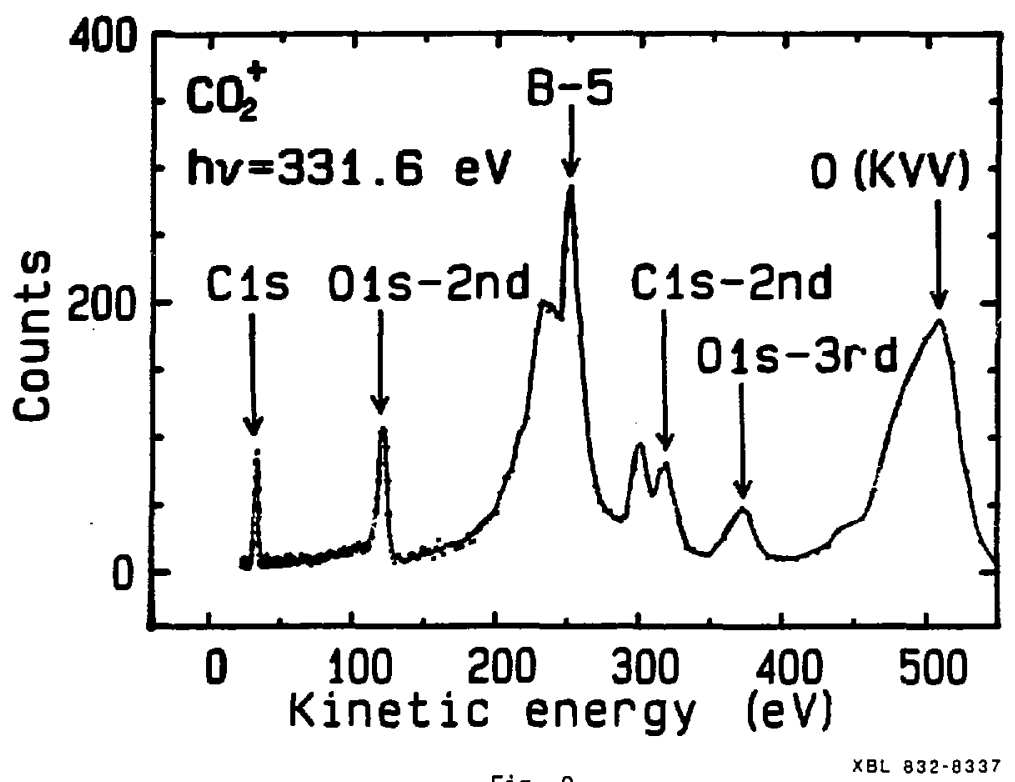

Fig. 9 
182
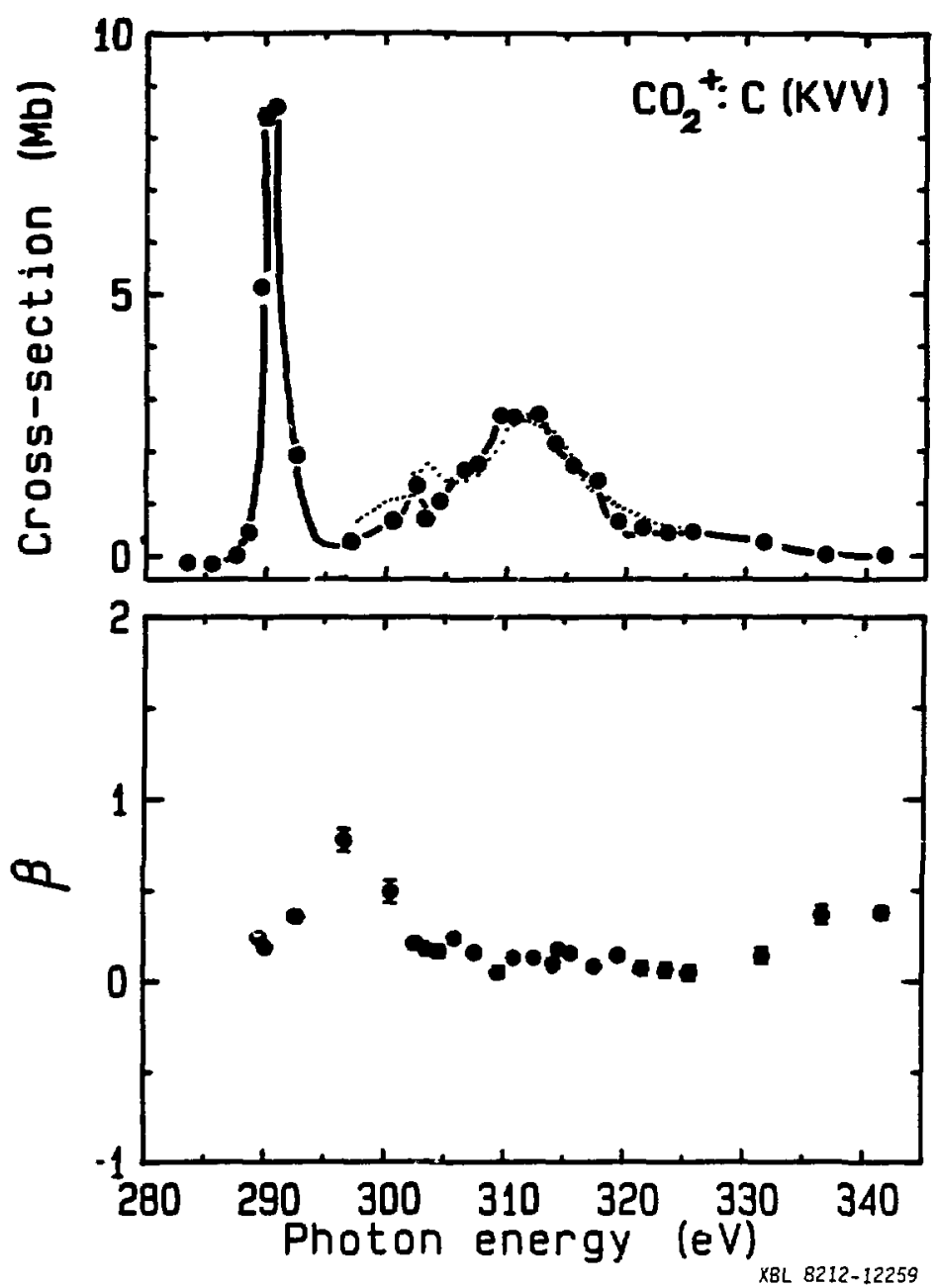

Fig. 10 
183
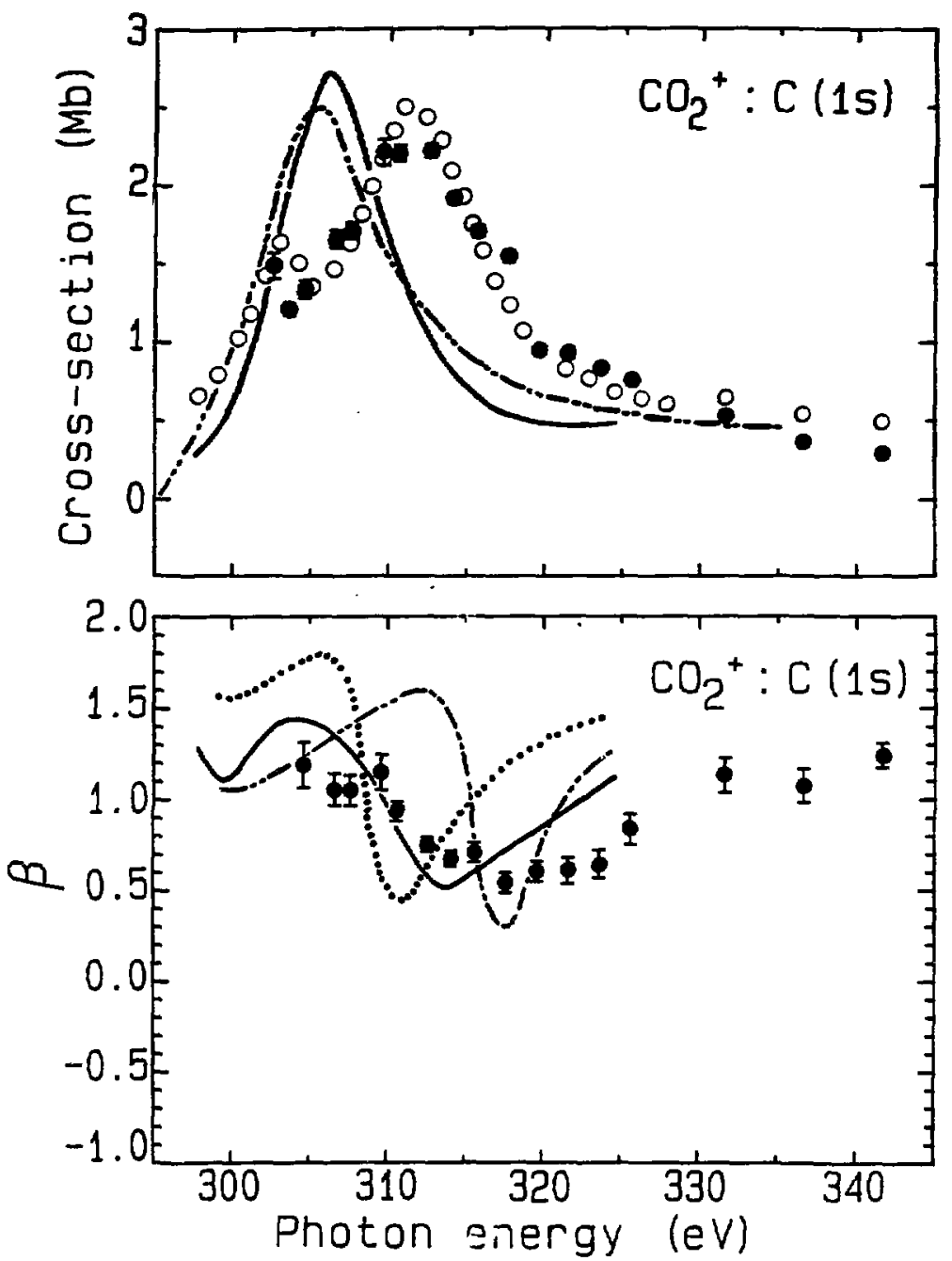

Fig. 11 

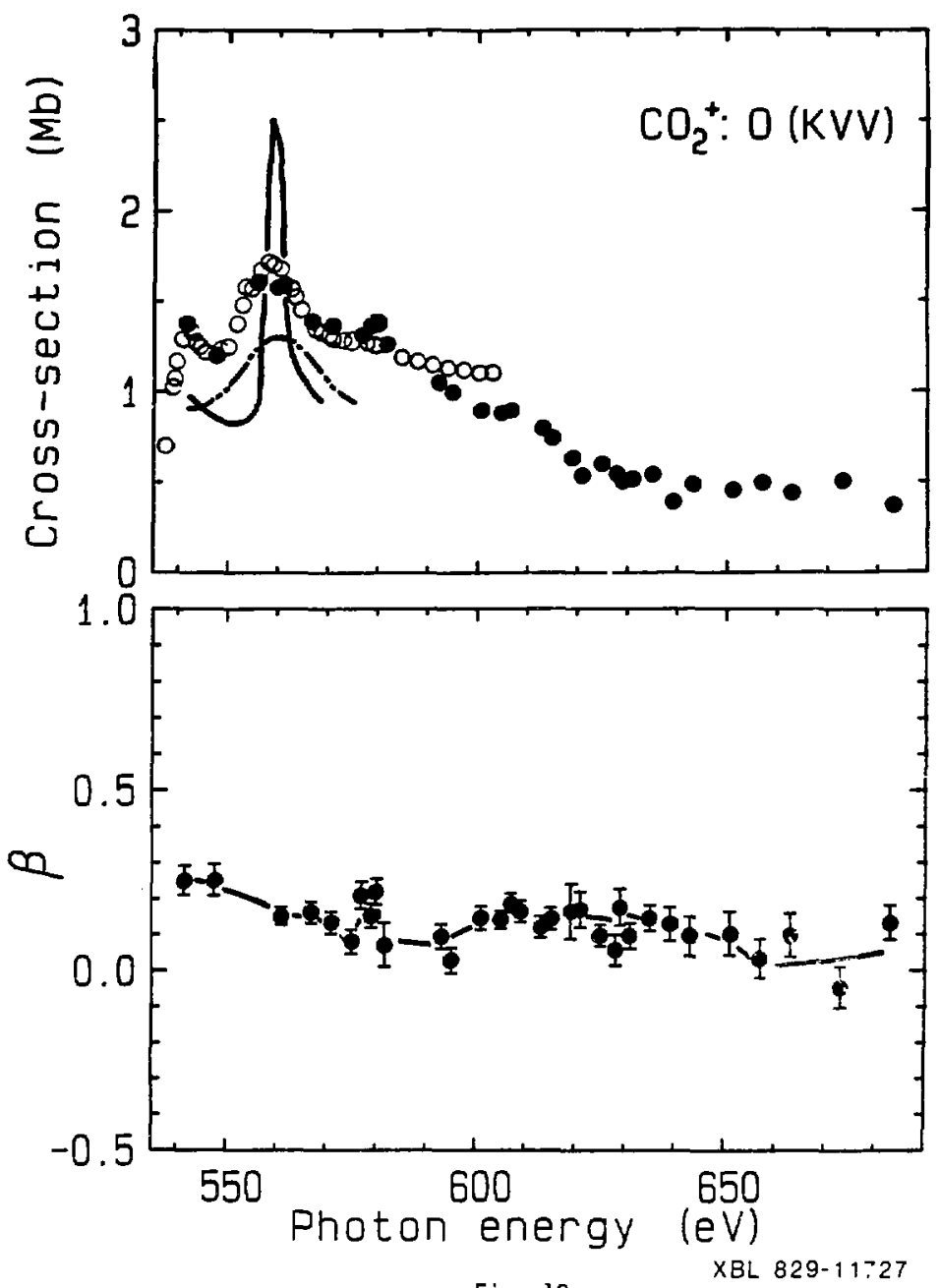

Fig. 12 


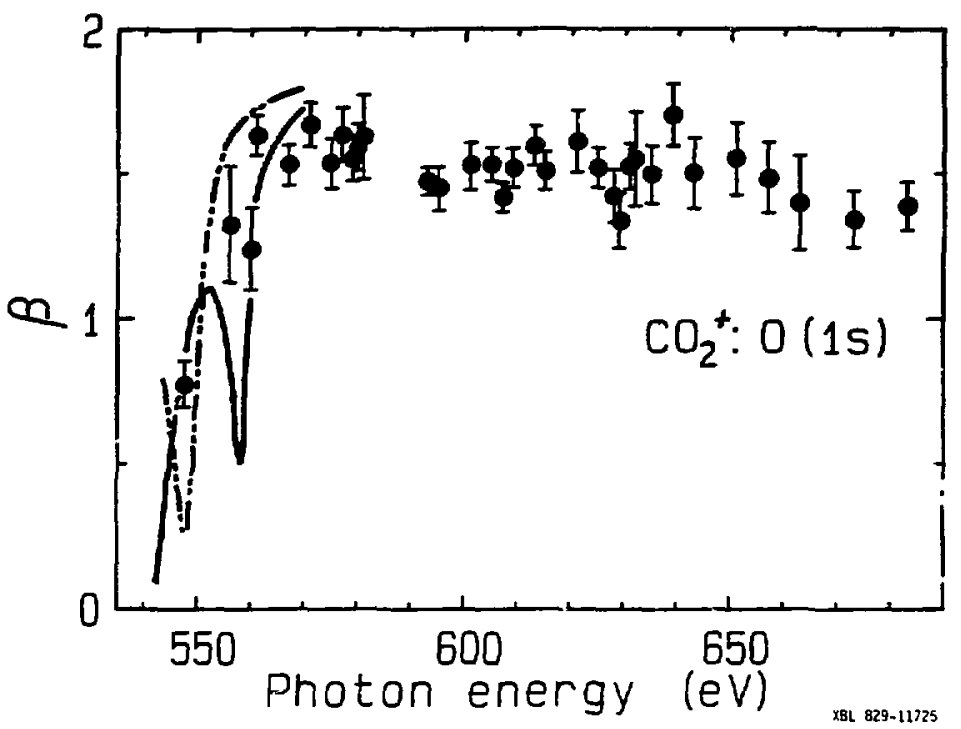

Fig. 13 


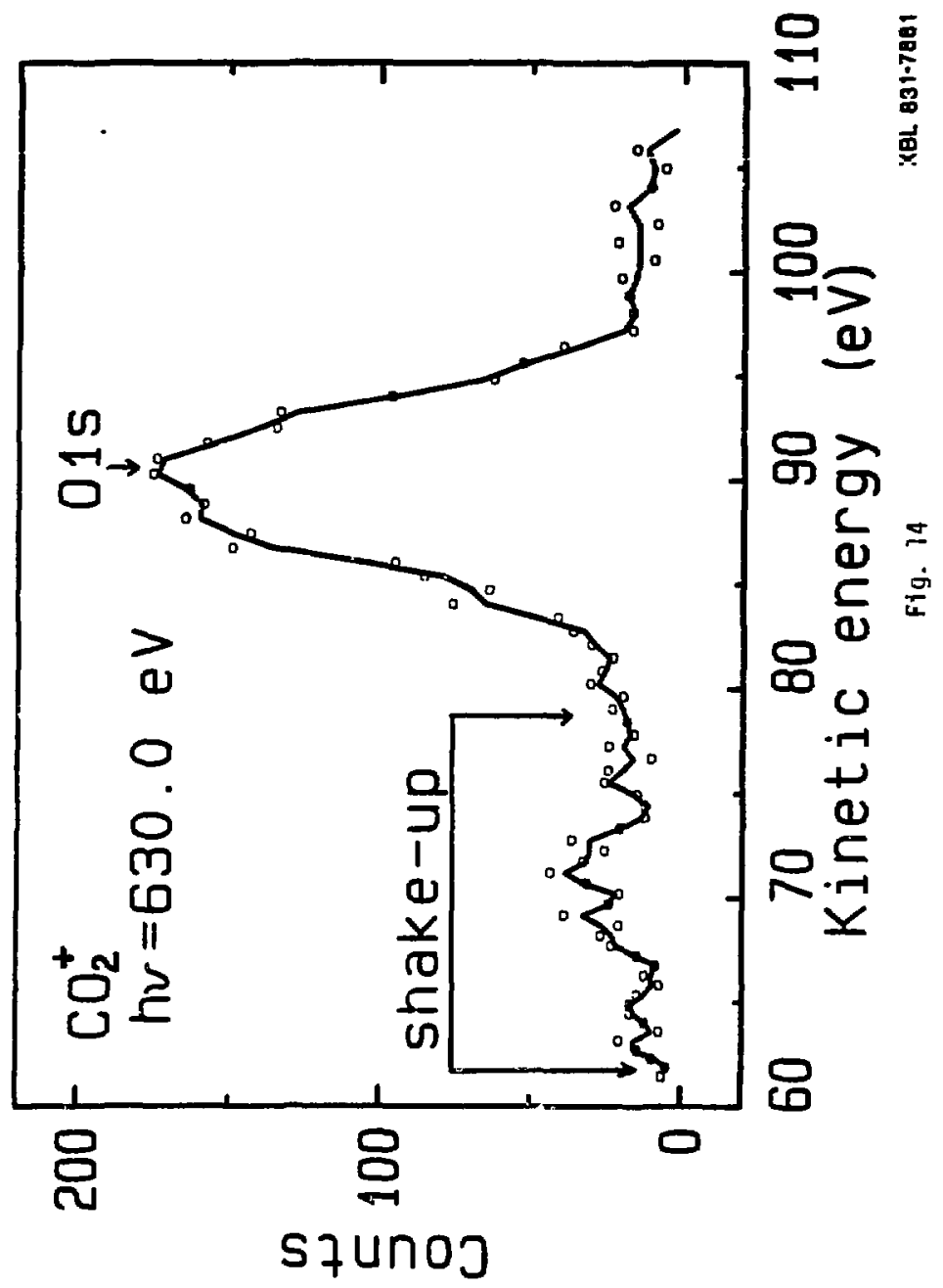




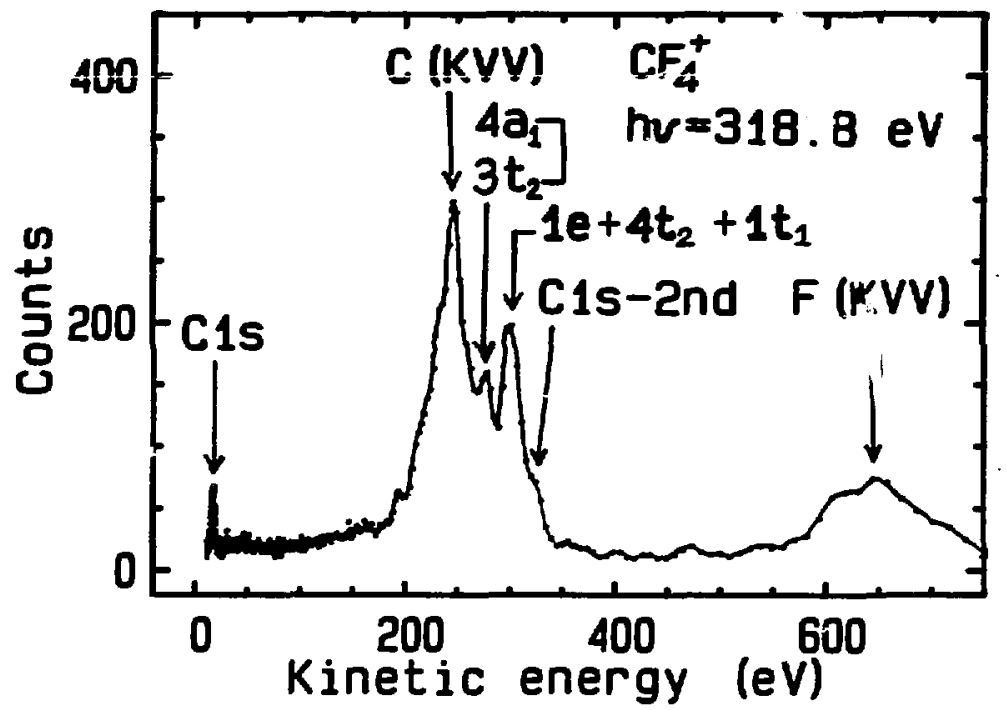

Fig. 15

X8L 832-8334 
188
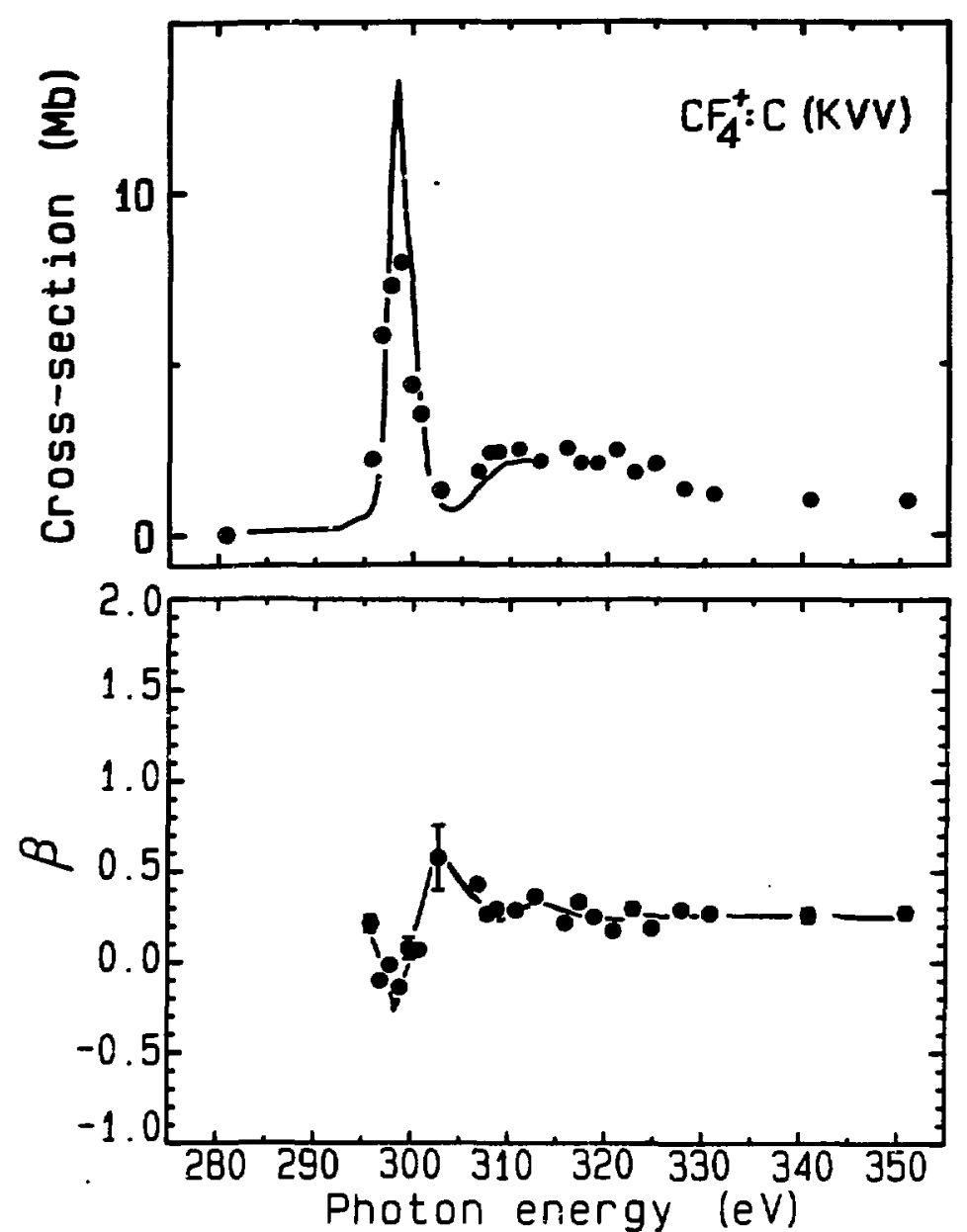

Fig. 16 

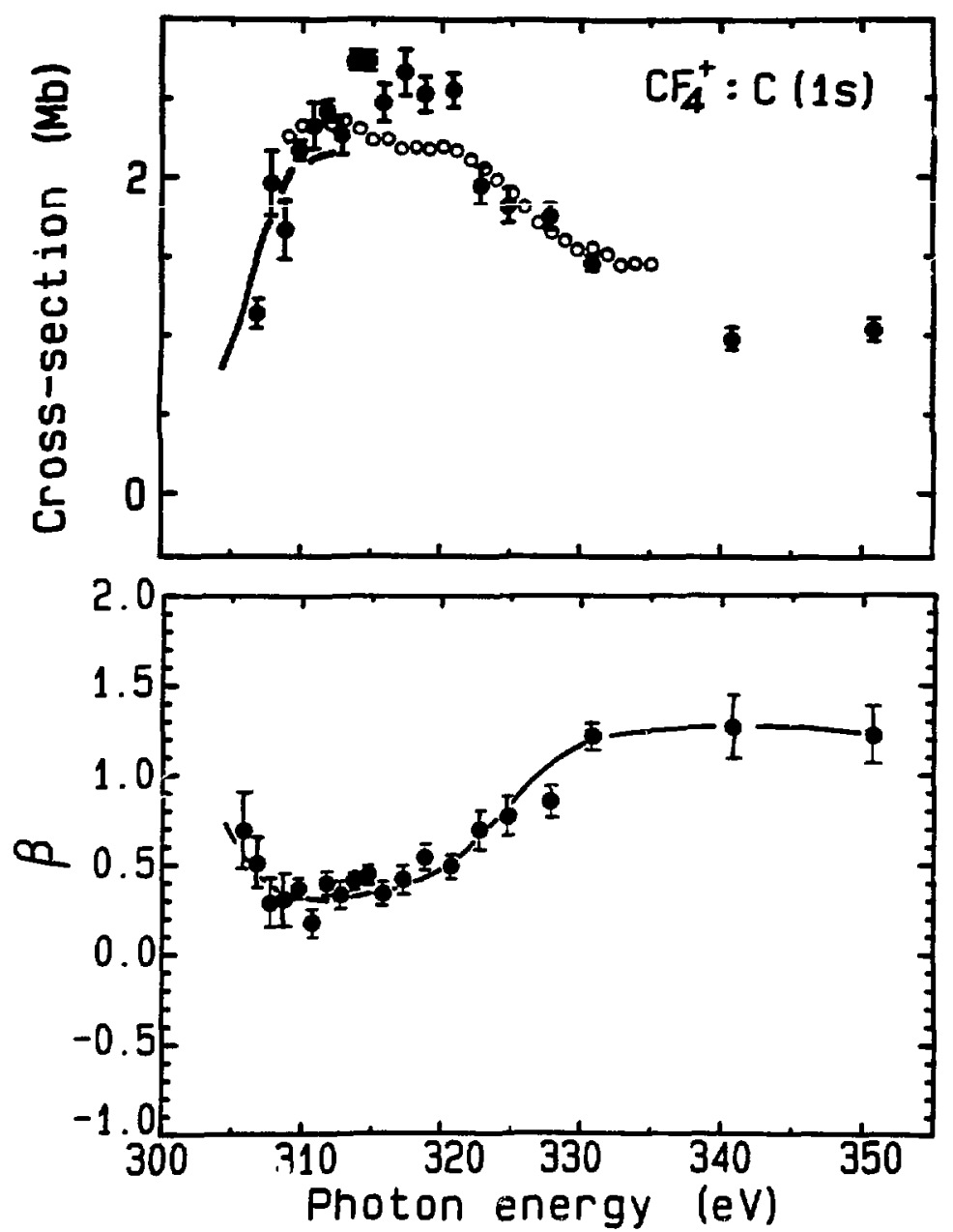

Fig. 17 


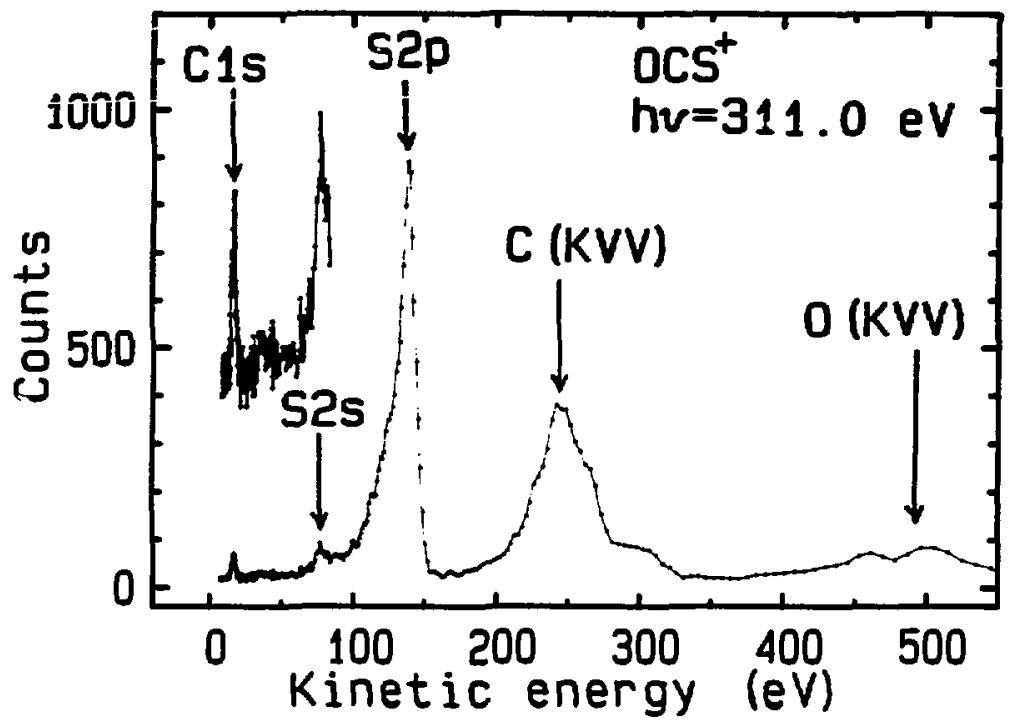

Fig. 18 


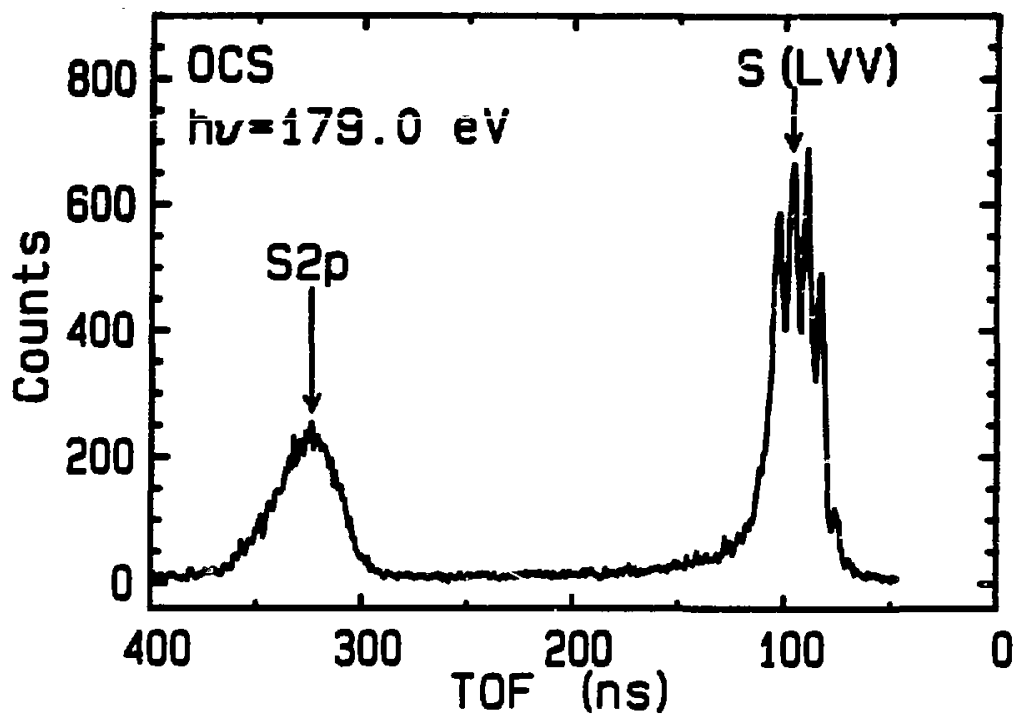

Fig. 19

X日L $832-8336$ 
192
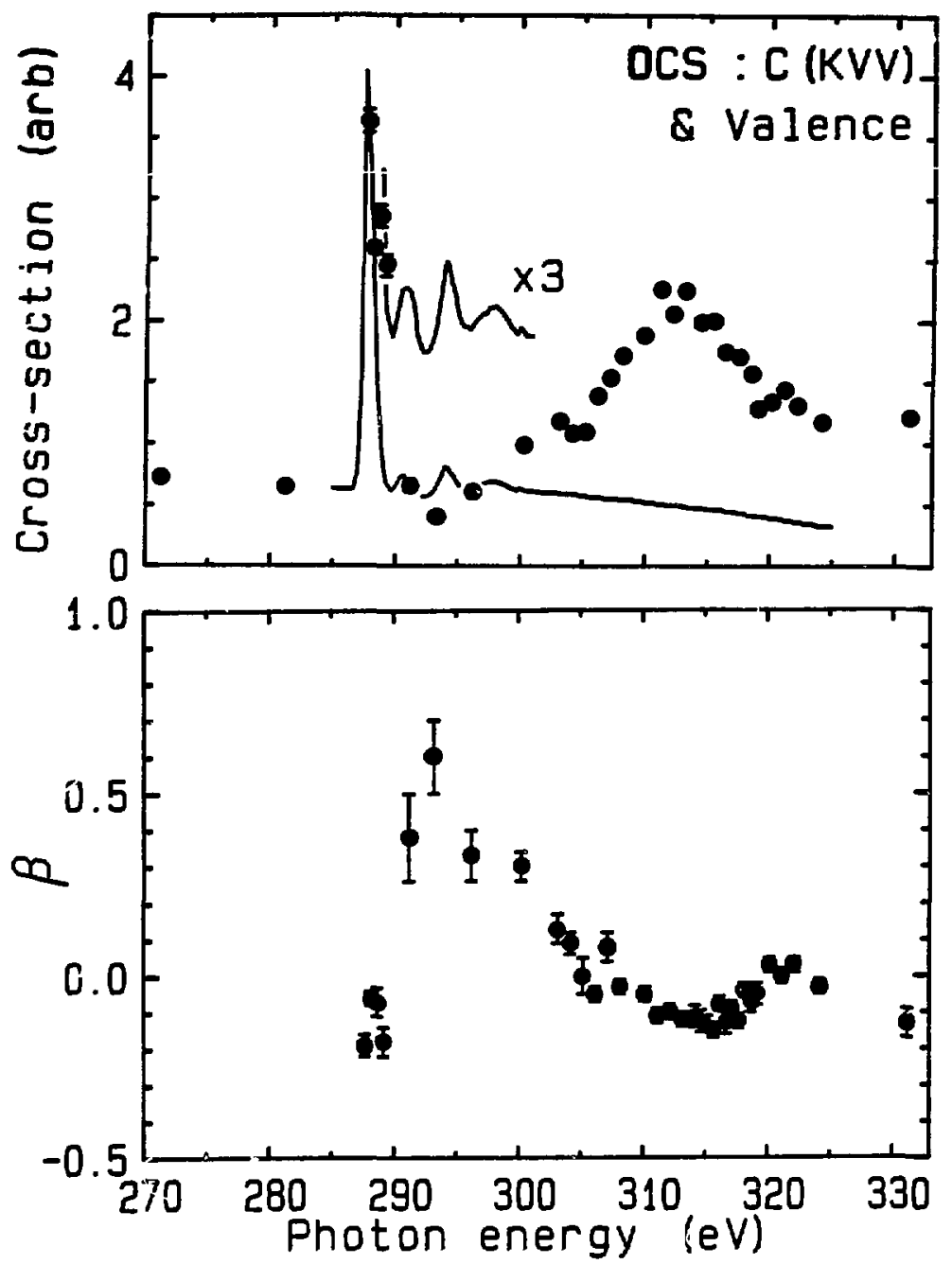

Fig. 20

XBL 833-8921 

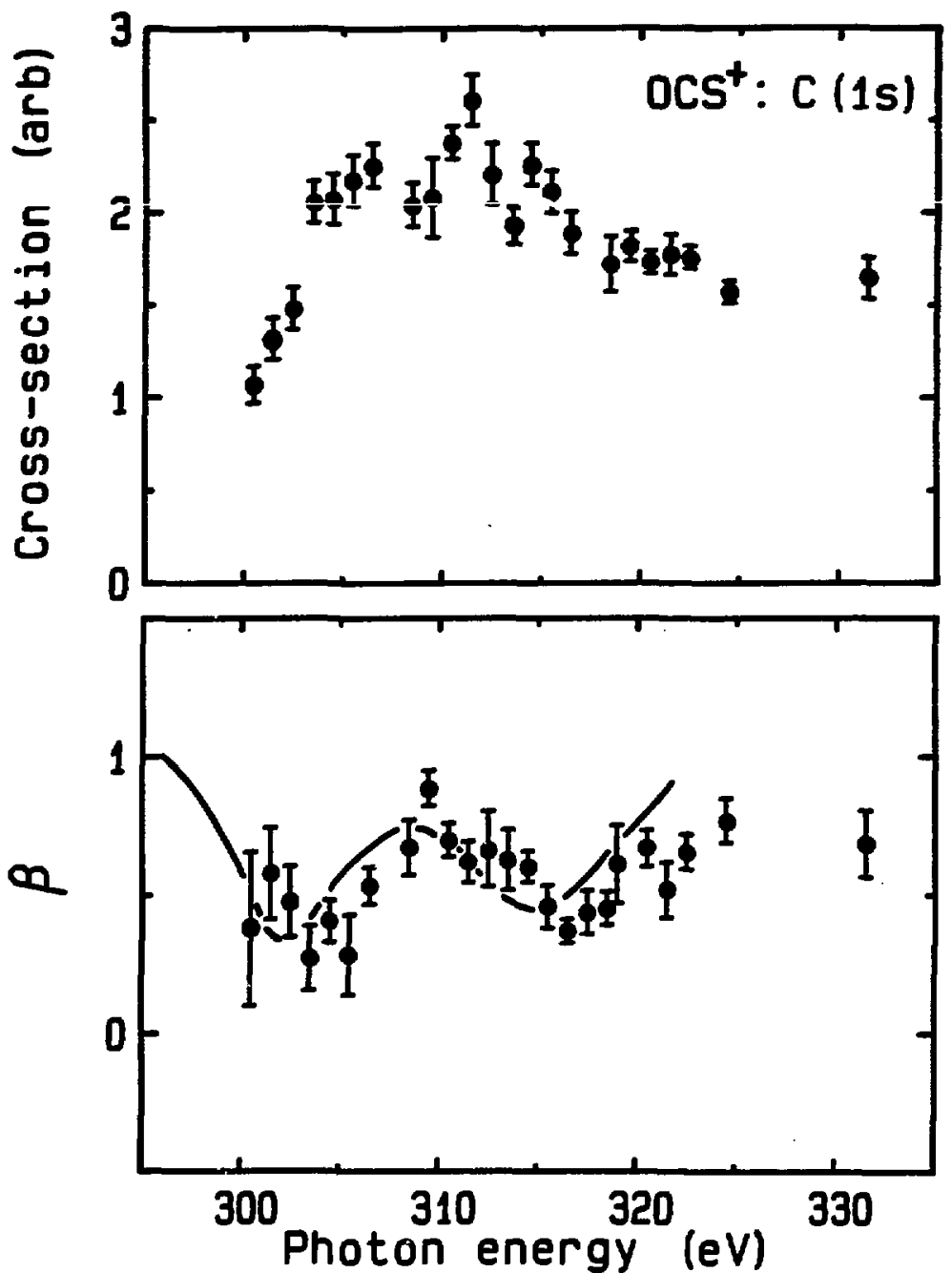

Fig. 21 

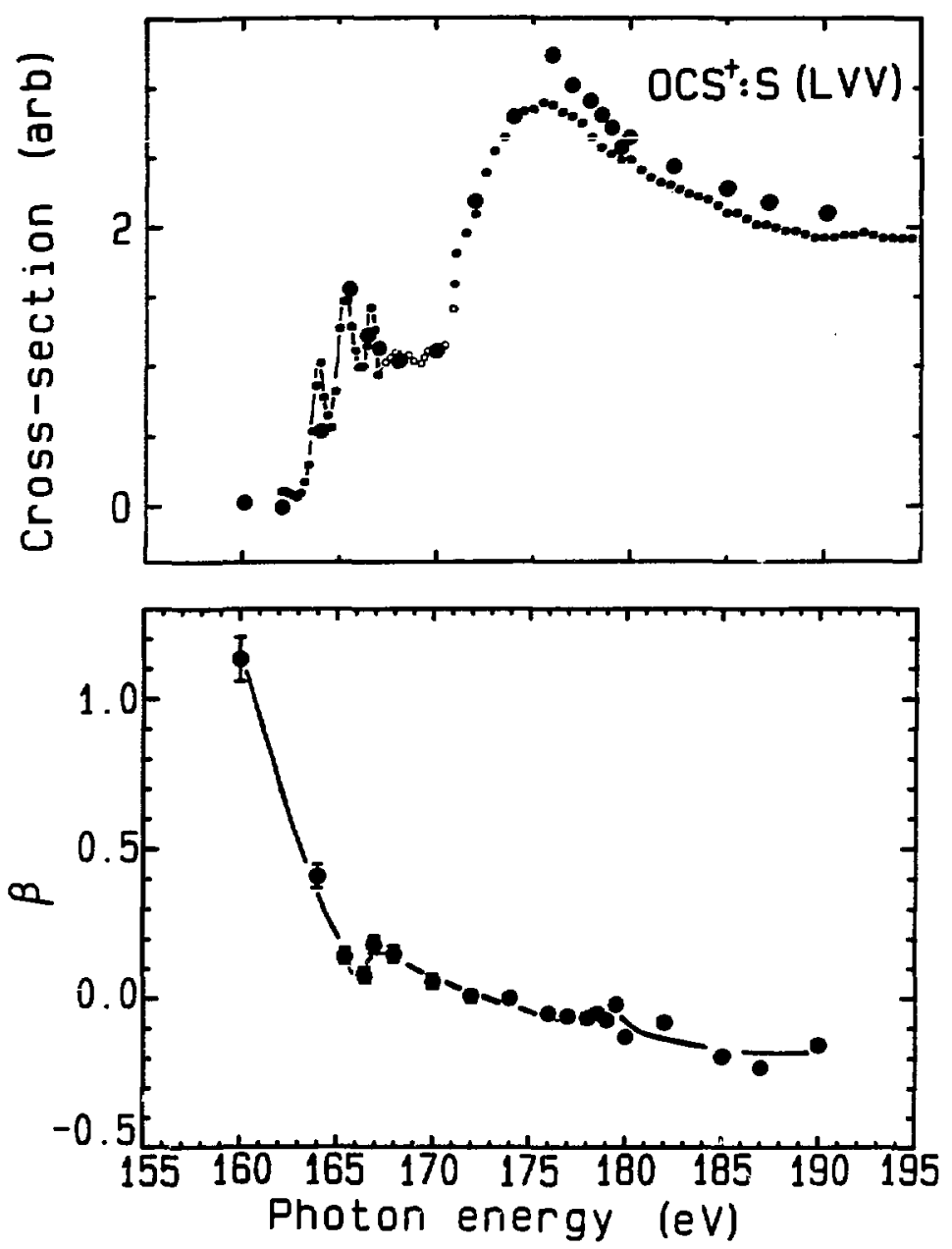

fig. 22 

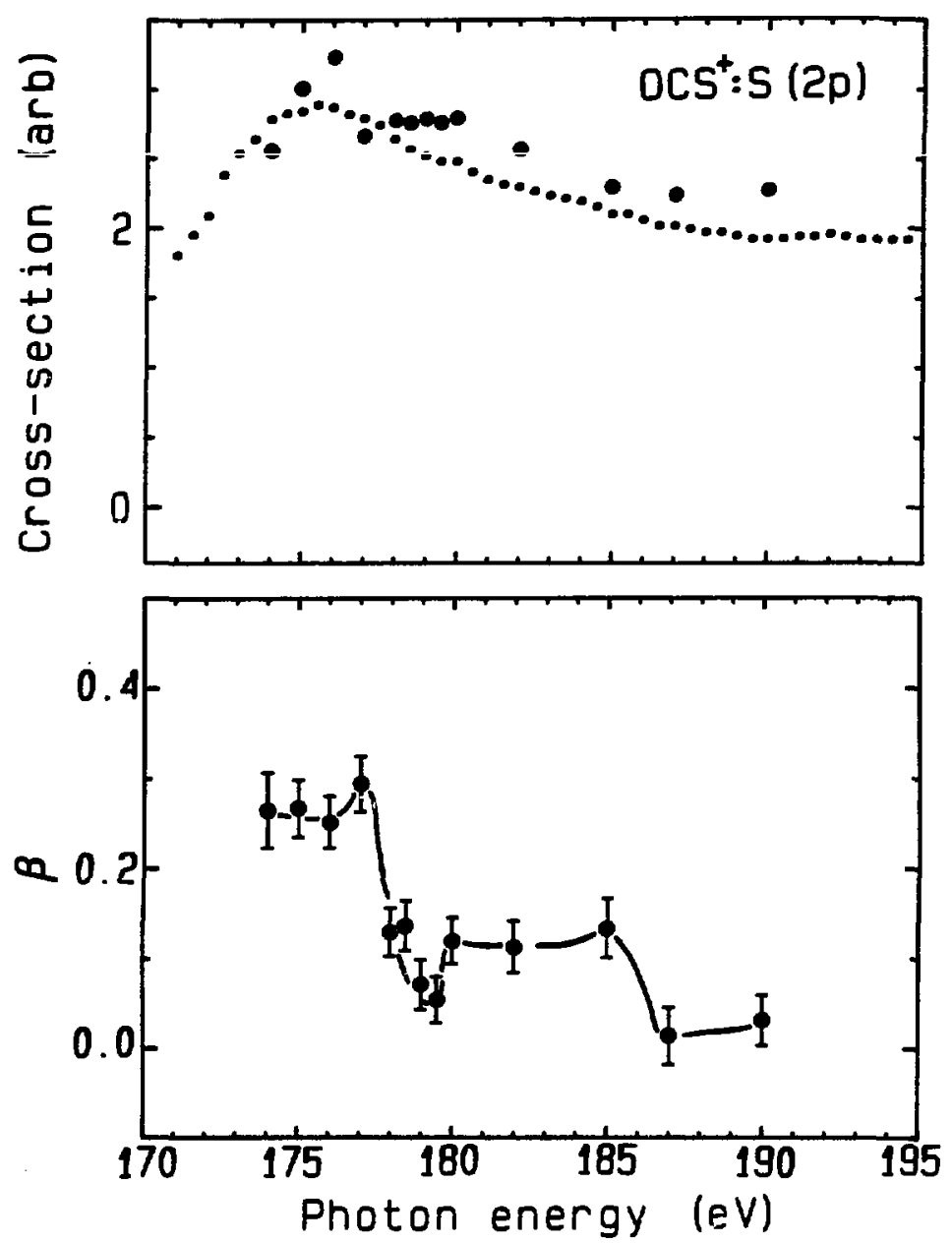

Fig. 23

XBL 828-11145 


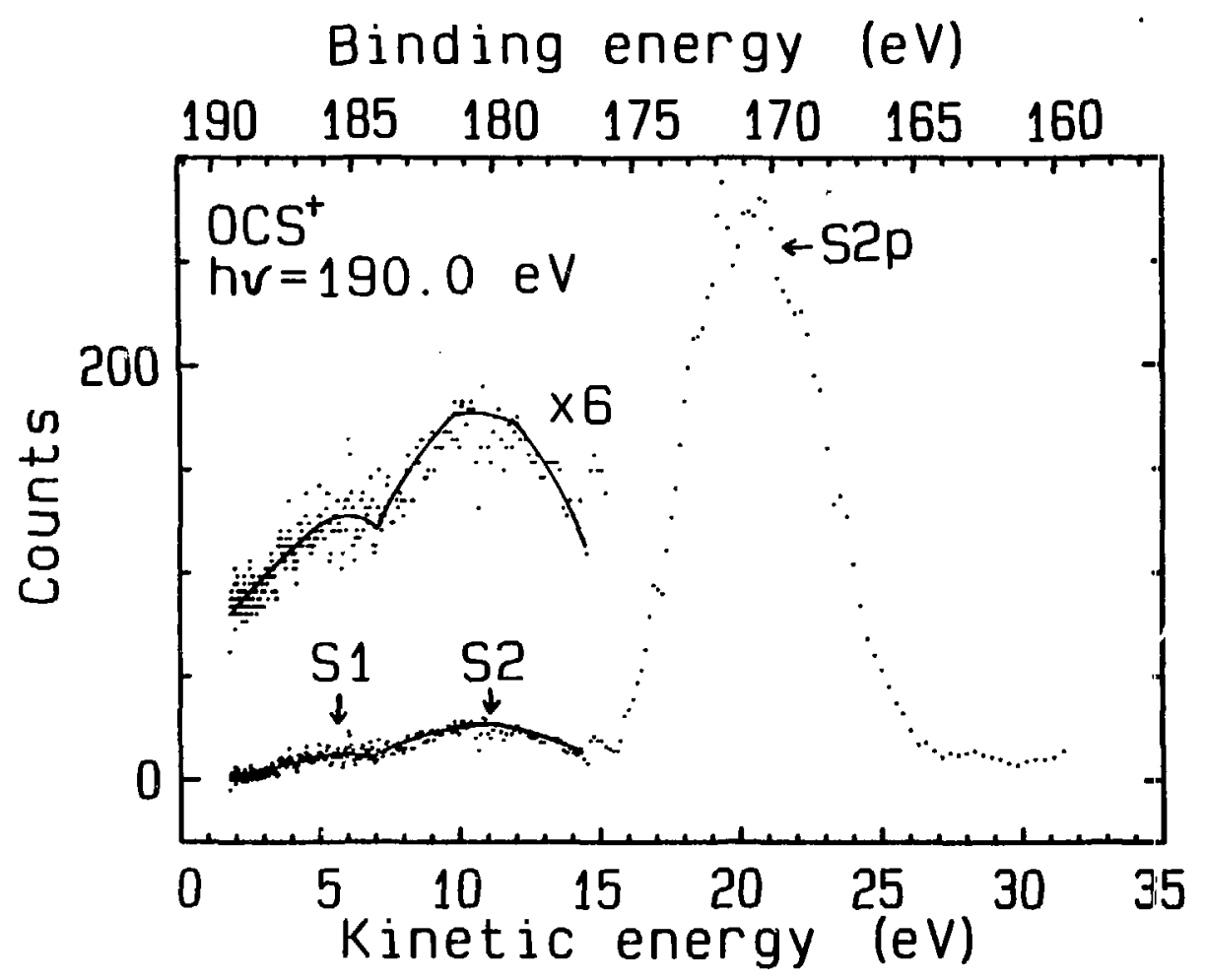

몽

Fig. 24

XBL $831-71180$ 
197

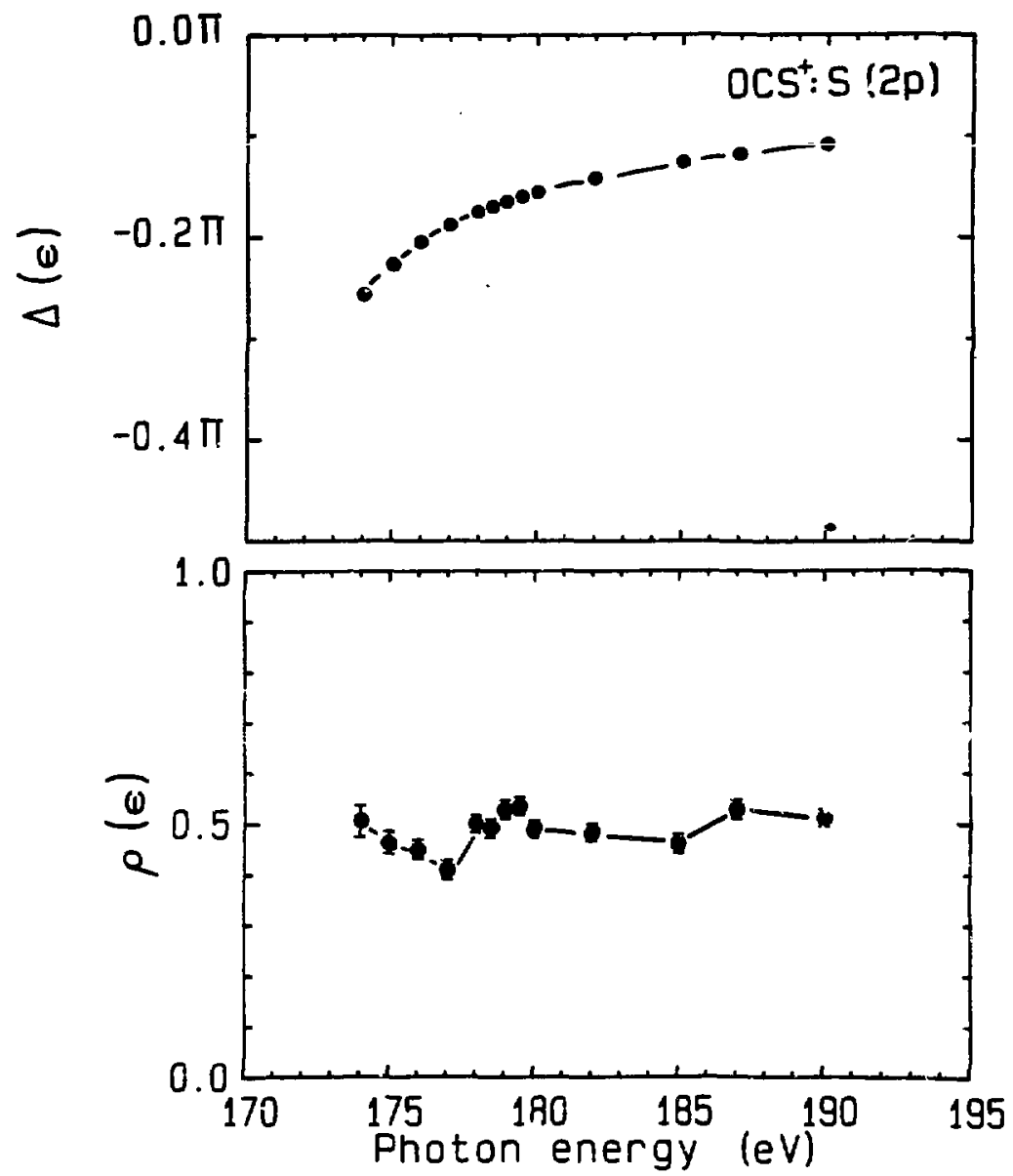

Fig. 25

XBL $831-7883$ 


\section{Acknowledgements}

The collaborative work herein required many hours of preparation as well as sustained support from many individuals. I praise these people for their efforts in providing expertise, love, friendsnip toward the culmination of my graduate career.

I would like to thank ny research director, David A. Shirley, for his support, for his scientific advice, and for allowing me the opportunity to investigate my own experimental interests.

I would like to acknowledge several co-workers for making the ToF "project" successful. Steve Southworth, Paul Kobrin, and Dennis Lindle were students whom I have worked with the longest period of time. Bill Brewer, Shige Owaki, Uwe Becker, and Hans Kerkhoff were visitors in our research laboratory. I tha:ak Phil Heimann and Trish Ferrett, who are new students in our research group, for their assistance.

I would also like to thank Dennis Trevor, John Barton, and Charlie Bahr for providing helpful hunor, suggestions, and information.

I would like to acknowledge support from the people in the mechanical engineering and machinists groups in building $70 \mathrm{~A}, T I D$, Joe Katz, and George Gabor. I would also like to thank the staff at SSRL for all of their assistance.

I would like to acknowledge Barbara Moriguchi, Wini Heppler, and the other members of the Shirley group for their friendship and assistance. I thank Deborah Colbert, Jean Wolslegel, and June de la Vergne for 
their work in editing, and printing the final copy of this manuscript. This work was supported by the Director, Office of Energy Research, Office of Basic Energy Sciences, Chenical Sciences Division of the U.S. Department of Energy under Contract MO. DE-ACO3-76SF00098. It was performed at the Stanford Synchrotron Radiation Laboratory, wich is supported by the MSF through the Division of Materials Research. Department of Energy. Any conelusions or opinions expressed in this report represent sokly those of the author(s) and not necessarily those of The Regents of the Unjversily of California. the Lawresce Berkeley Laboratory or the Department of Energy.

Reference to a company or product name does not imply approval or recommendation of the product by the University of California or the U.S. Department of Energy to the exclusion of others that may be suitable. 